\title{
OPEN SOURCE TOOLKIT FOR REENTRY OBJECT MODELING
}

\author{
A Thesis \\ presented to \\ the Faculty of California Polytechnic State University, \\ San Luis Obispo
}

\author{
In Partial Fulfillment \\ of the Requirements for the Degree \\ Master of Science in Aerospace Engineering
}

by

Christopher L. Ostrom II

December 2015 
(C) 2015

Christopher L. Ostrom II

ALL RIGHTS RESERVED 
COMMITTEE MEMBERSHIP

TITLE:

Open Source Toolkit for Reentry Object Modeling

AUTHOR: $\quad$ Christopher L. Ostrom II

DATE SUBMITTED: December 2015

COMMITTEE CHAIR: Kira J. Abercromby, Ph.D.

Assistant Professor of Aerospace Engineering

COMMITTEE MEMBER: David D. Marshall, Ph.D.

Professor of Aerospace Engineering

COMMitTeE MEMBER: Piyush M. Mehta, Ph.D.

Marie Curie Fellow at University of Strathclyde

COMMITTEE MEMBER: Kim A. Shollenberger, Ph.D.

Professor of Mechanical Engineering 


\section{ABSTRACT \\ Open Source Toolkit for Reentry Object Modeling \\ Christopher L. Ostrom II}

Predicting the mass, position, and velocity of an object during its reentry are critical to satisfy NASA and ESA requirements. This thesis outlines a 3-D orbit and mass determination system for use on low earth orbit as applicable to general objects, of various material and size. The solution uses analytical models to calculate heat flux and aerodynamic drag, with some basic numerical models for simple orbit propagation and mass flow rate due to ablation. The system outlined in this thesis currently provides a framework for rough estimates of demise altitude and final mass, but also allows for many potential accuracy and speed improvements.

77 aerospace materials were tested, in solid spheres, cubes, and cylinders; it was found that materials with low latent heat of fusion (less than $10 \frac{\mathrm{kJ}}{\mathrm{kg} \cdot \mathrm{K}}$ ) demise before reaching the ground, while materials with higher melting point temperatures (over 1200K), high specific heats, and high latent heat of fusion (over $30 \frac{\mathrm{kJ}}{\mathrm{kg} \cdot \mathrm{K}}$ ) lose small amounts of mass before hitting the ground at speeds of $200-300 \frac{\mathrm{m}}{\mathrm{s}}$. The results of this thesis code are validated against NASA's Debris Assessment System (DAS), specifically the test cases of Acrylic, Molybdenum, and Silver. 


\section{ACKNOWLEDGMENTS}

Foremost, I would like to express my sincere gratitude to my advisor Dr. Kira Abercromby for the continuous support of my study and research, for her patience, motivation, enthusiasm, and knowledge. Her guidance helped me during all the research and writing of this thesis.

Besides my advisor, I would like to thank the rest of my thesis committee: Dr. David Marshall, Dr. Piyush Mehta, and Dr. Kim Shollenberger, for their encouragement, insightful comments, and hard questions.

Last but not least, I would like to thank my family: my parents Chris and Linda, and fiancée Hélène, for their unwavering support. 


\section{TABLE OF CONTENTS}

Page

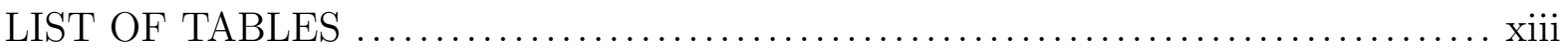

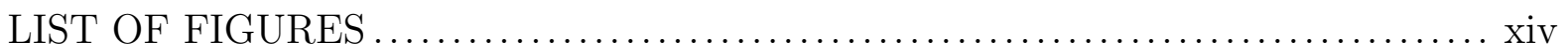

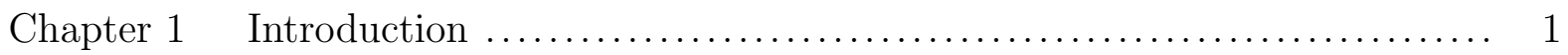

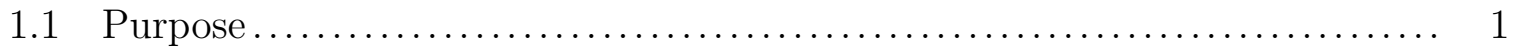

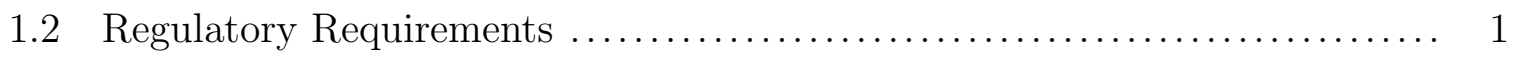

1.3 Reentry Modeling ........................................... 2

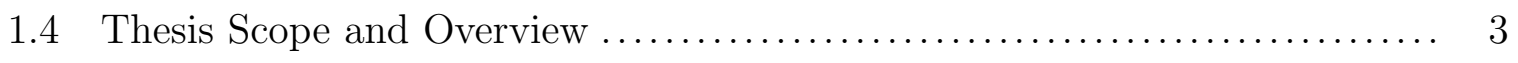

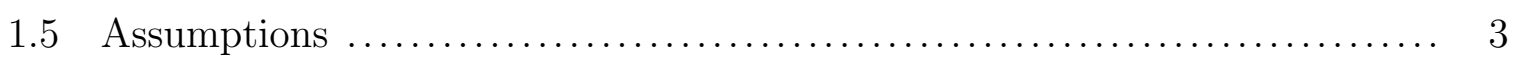

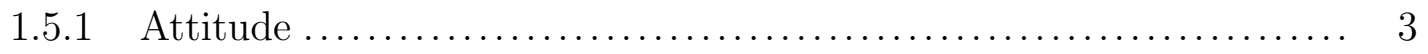

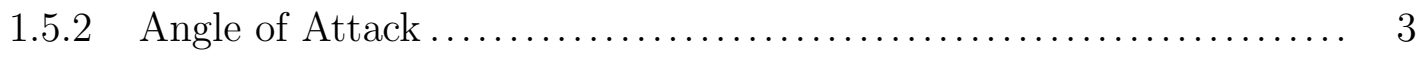

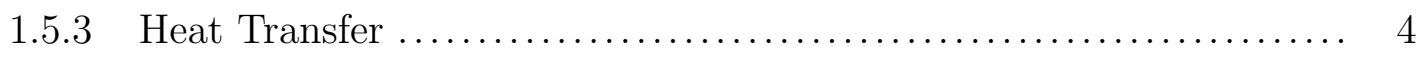

1.5.4 Ablation ............................................. 4

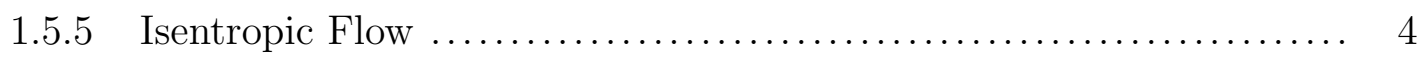

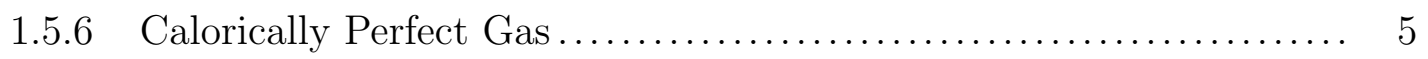

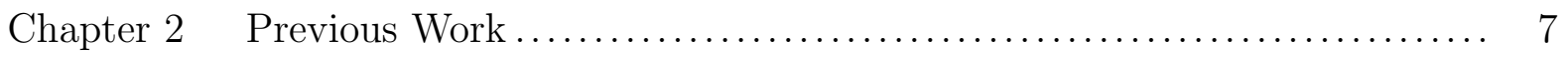

2.1 Reentry Analysis Tools . . . . . . . . . . . . . . . . . . . . . . . . . . . . . 7

2.1.1 Debris Assessment Software (DAS) $\ldots \ldots \ldots \ldots \ldots \ldots \ldots \ldots \ldots \ldots \ldots$

2.1.1.1 Mission Editor .............................. 7

2.1.1.2 Requirement Assessment $\ldots \ldots \ldots \ldots \ldots \ldots \ldots \ldots \ldots \ldots \ldots . \ldots$ 
2.1.1.3 Material Database $\ldots \ldots \ldots \ldots \ldots \ldots \ldots \ldots \ldots \ldots \ldots \ldots \ldots \ldots$

2.1.1.4 Science and Engineering Utilities .................... 8

2.1.2 Object Reentry Survival Analysis Tool (ORSAT) ................ 9

2.1.3 Spacecraft Atmospheric Reentry \& Aerothermal Breakup (SCARAB) 9

2.2 Comparison of DAS, ORSAT, and SCARAB $\ldots \ldots \ldots \ldots \ldots \ldots \ldots \ldots \ldots \ldots \ldots \ldots \ldots \ldots \ldots \ldots$

Chapter $3 \quad$ Models ................................................... 14

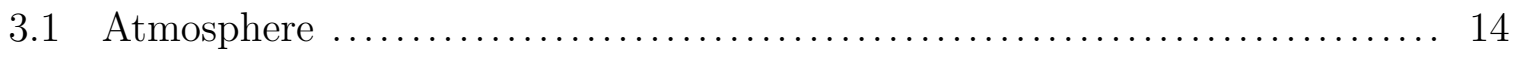

3.1.1 Global Reference Atmospheric Model (GRAM) ................. 14

3.1.2 Mass Spectrometer and Incoherent Scatter Radar (MSISE) ....... 15

3.1.3 U.S. Standard Atmosphere.................................. 15

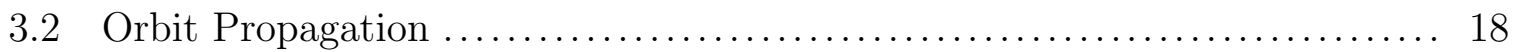

3.2.1 Runge-Kutta Methods .................................... 18

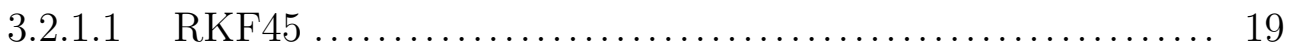

3.2.1.2 RK78 ................................... 20

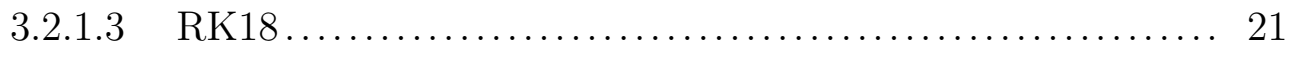

3.2.1.4 Step Size Control.................................. 22

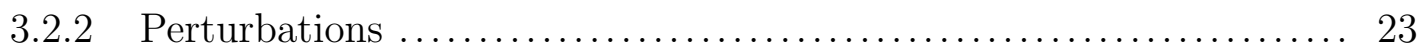

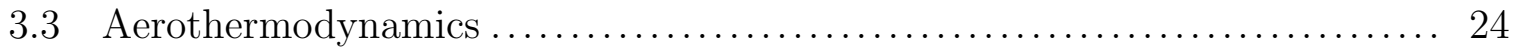

3.3.1 Analytical Models ....................................... 24

3.3.1.1 Atmospheric Drag ............................... 24

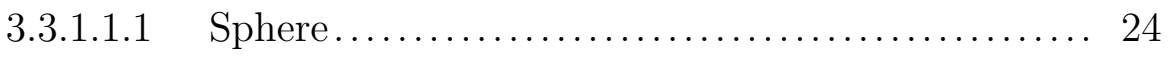

3.3.1.1.2 Cube ................................ 27

3.3.1.1.3 Cylinder................................ 28

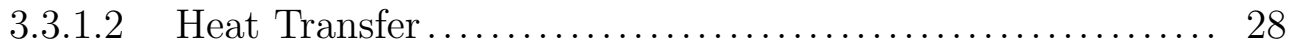

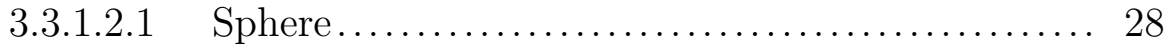

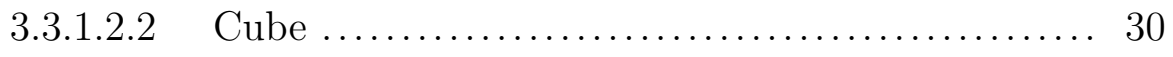

3.3.1.2.3 Cylinder................................. 31 
3.3.2 Direct Simulation Monte Carlo Method ....................... 32

3.3.2.1 Algorithm................................... 33

3.3.2.2 Applications to Atmospheric Entry Flows.............. 34

3.3.2.3 Coupling DSMC and Navier-Stokes.................. 35

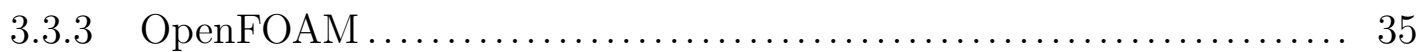

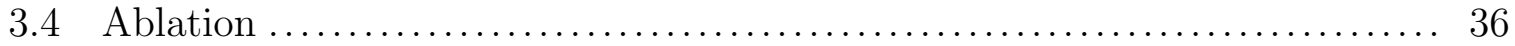

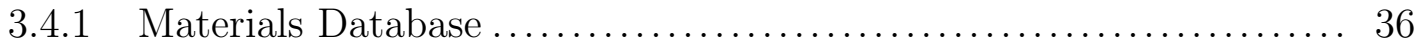

3.4.2 Ablation Modeling...................................... 36

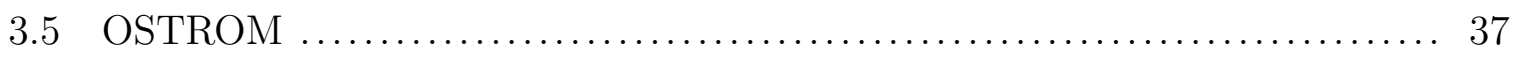

3.5.1 Algorithm .......................................... 39

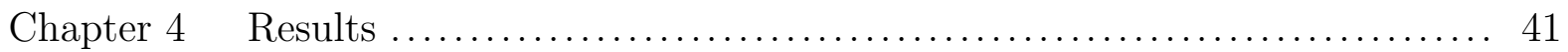

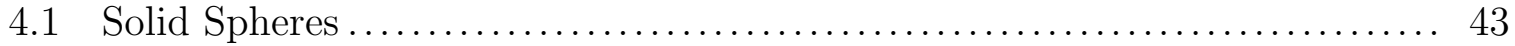

$4.1 .1 \quad$ Acrylic ................................................ 44

4.1 .2 Molybdenum ......................................... 45

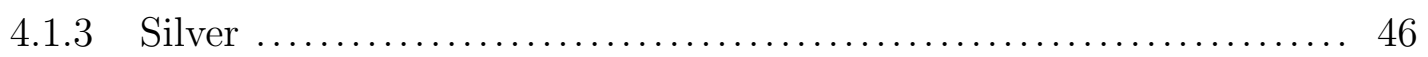

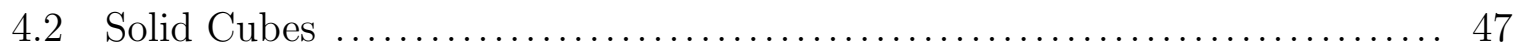

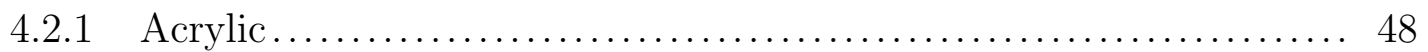

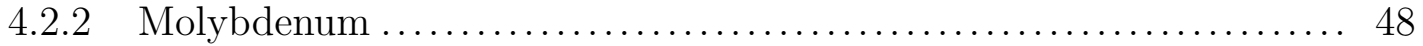

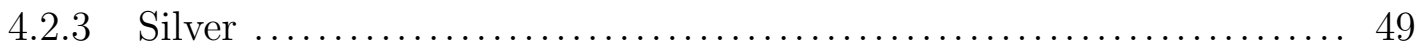

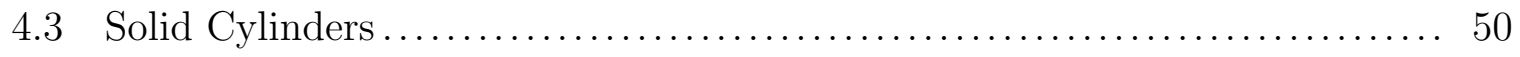

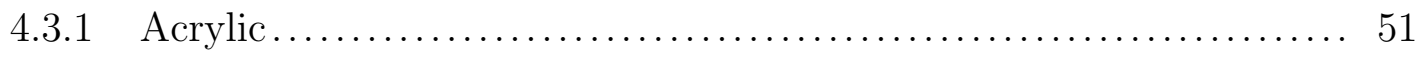

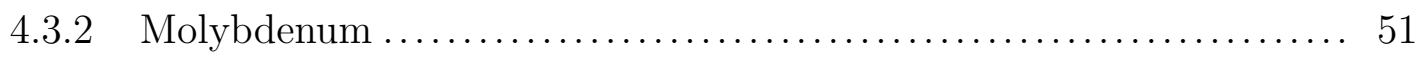

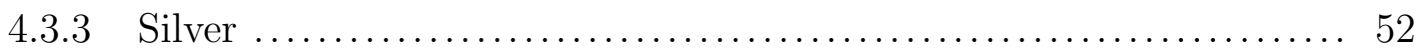

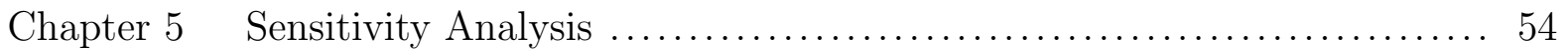

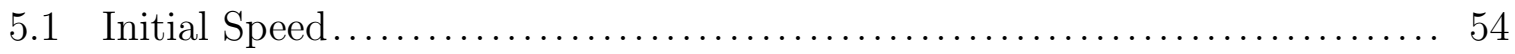

5.1 .1 Downrange Distance.................................. 55 


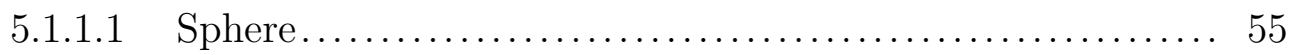

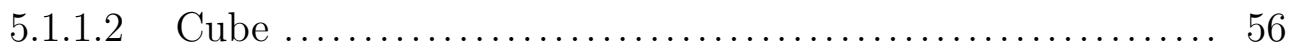

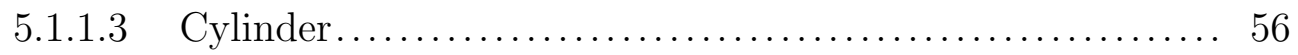

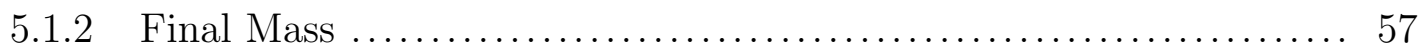

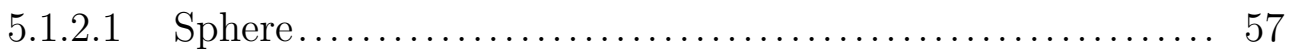

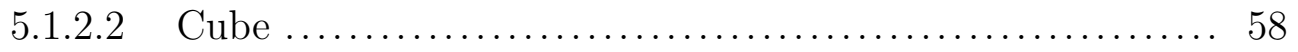

$5.1 .2 .3 \quad$ Cylinder....................................... 59

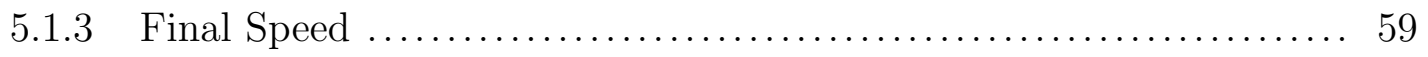

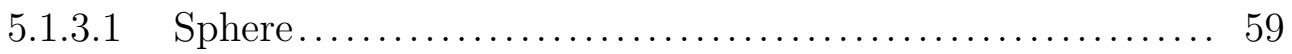

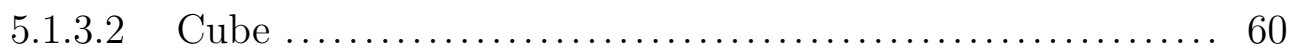

$5.1 .3 .3 \quad$ Cylinder $\ldots \ldots \ldots \ldots \ldots \ldots \ldots \ldots \ldots \ldots \ldots \ldots \ldots \ldots \ldots \ldots, 61$

5.1.4 Demise Altitude ........................................ 62

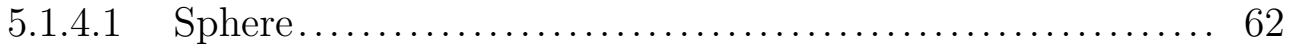

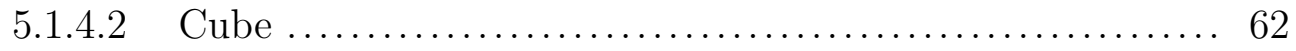

$5.1 .4 .3 \quad$ Cylinder....................................... 63

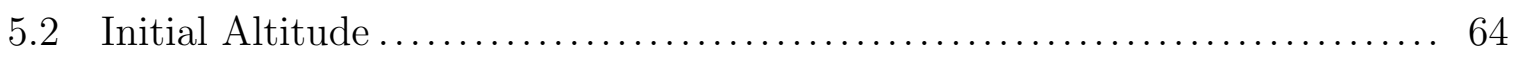

5.2 .1 Downrange Distance...................................... 64

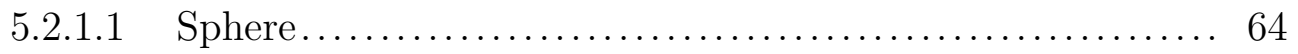

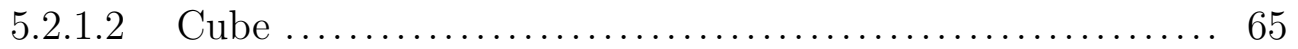

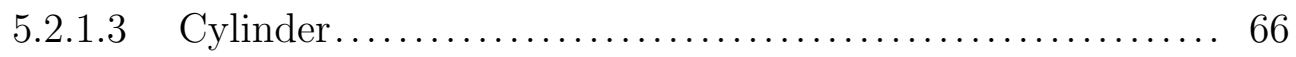

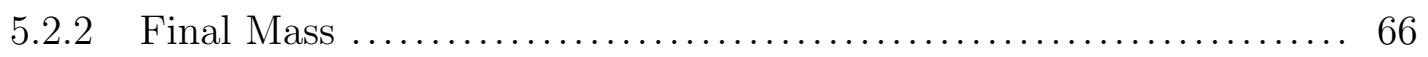

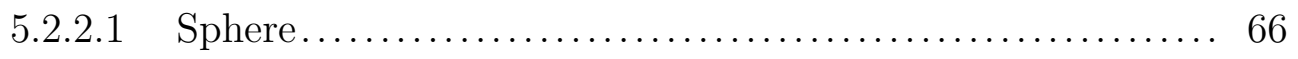

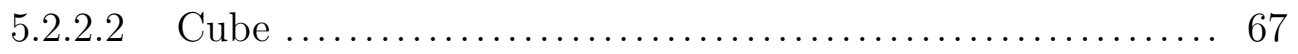

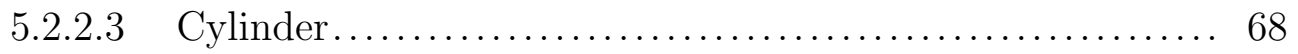

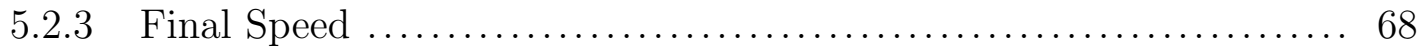

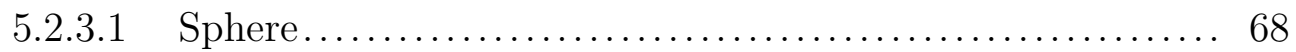

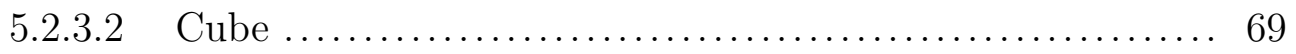




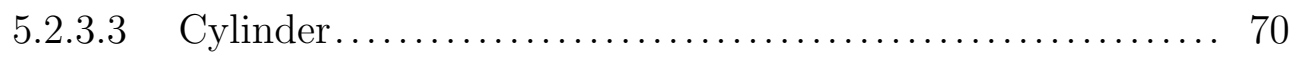

5.2.4 Demise Altitude ......................................... 70

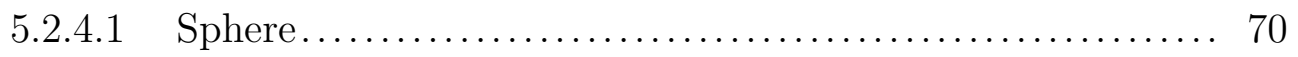

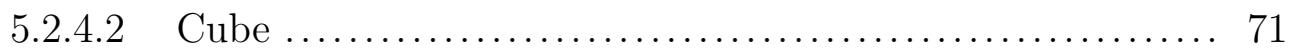

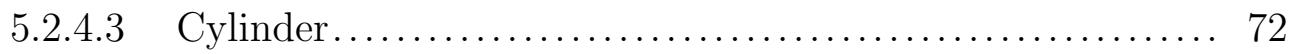

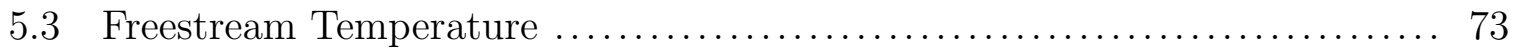

5.3.1 Downrange Distance....................................... 73

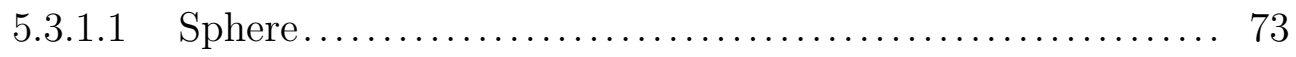

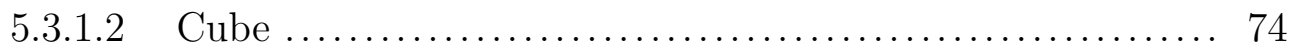

$5.3 .1 .3 \quad$ Cylinder....................................... 75

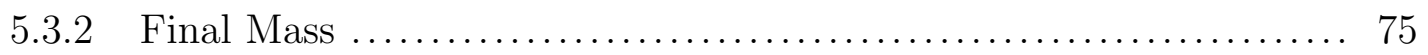

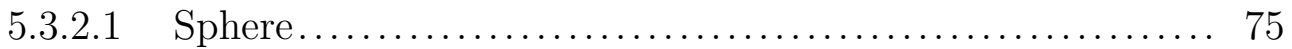

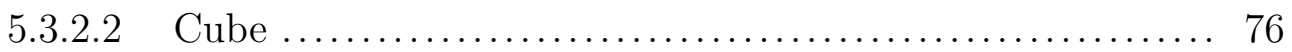

$5.3 .2 .3 \quad$ Cylinder....................................... 77

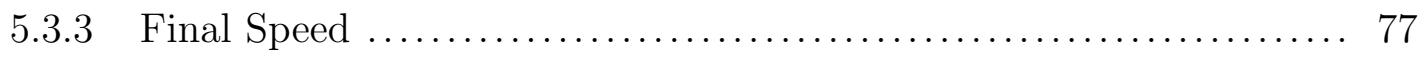

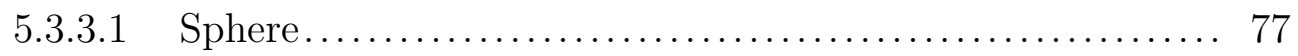

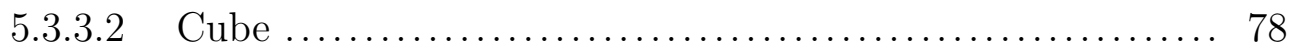

5.3.3.3 Cylinder...................................... 79

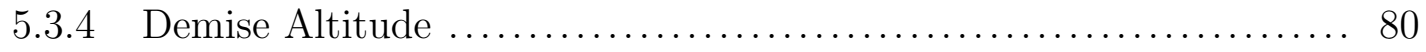

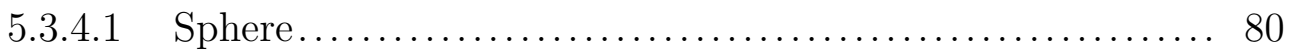

5.3.4.2 Cube ...................................... 81

$5.3 .4 .3 \quad$ Cylinder $\ldots \ldots \ldots \ldots \ldots \ldots \ldots \ldots \ldots \ldots \ldots \ldots \ldots \ldots \ldots, 81$

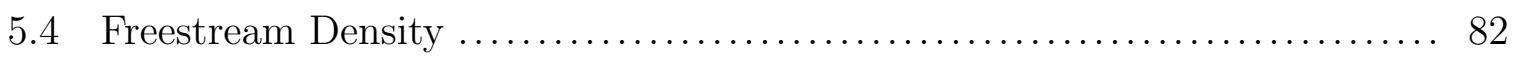

5.4 .1 Downrange Distance.................................... 82

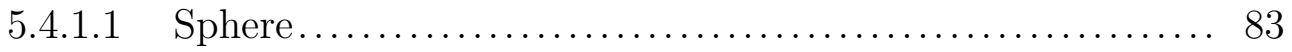

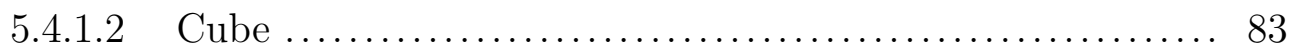

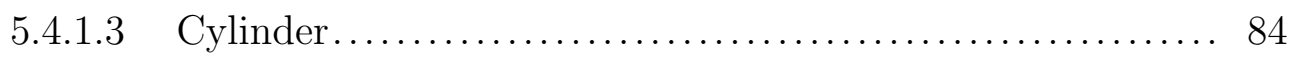




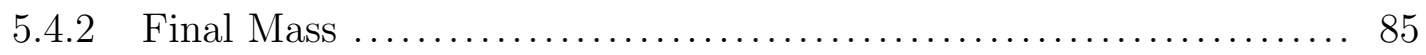

$5.4 .2 .1 \quad$ Sphere........................................ 85

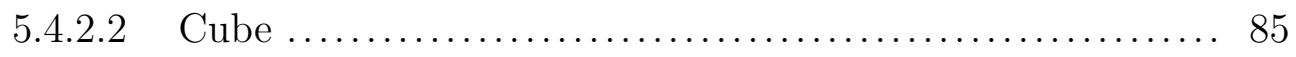

$5.4 .2 .3 \quad$ Cylinder........................................ 86

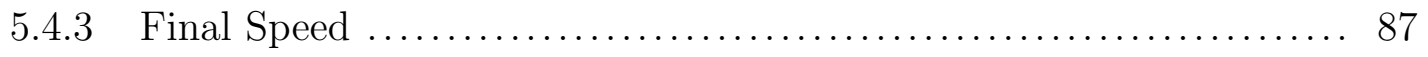

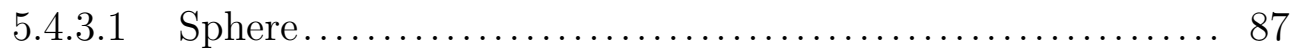

5.4.3.2 Cube ..................................... 87

$5.4 .3 .3 \quad$ Cylinder........................................ 88

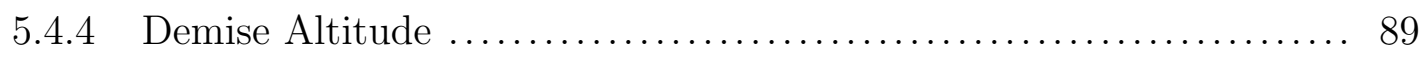

5.4.4.1 Sphere...................................... 89

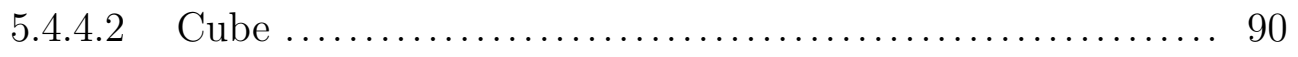

5.4.4.3 Cylinder...................................... 91

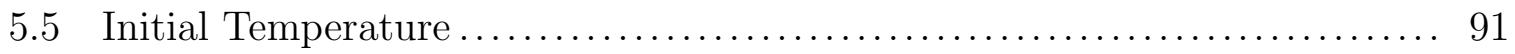

5.5 .1 Downrange Distance.................................... 91

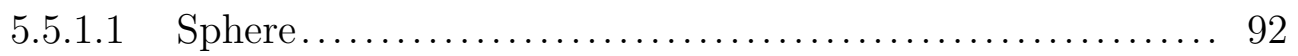

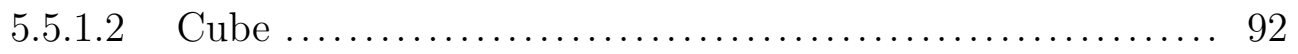

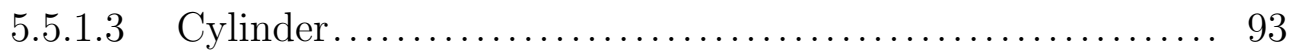

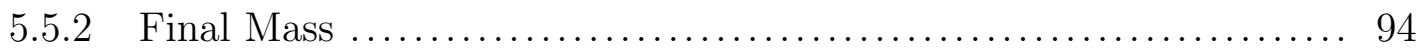

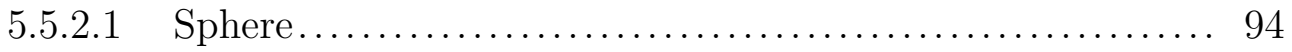

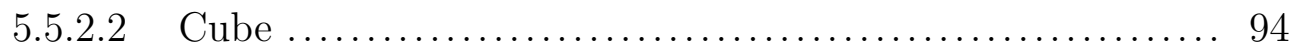

$5.5 .2 .3 \quad$ Cylinder........................................ 95

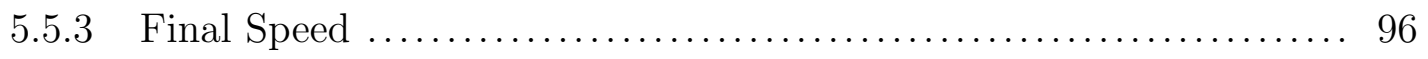

5.5.3.1 Sphere...................................... 96

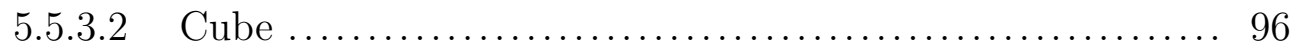

$5.5 .3 .3 \quad$ Cylinder......................................... 97

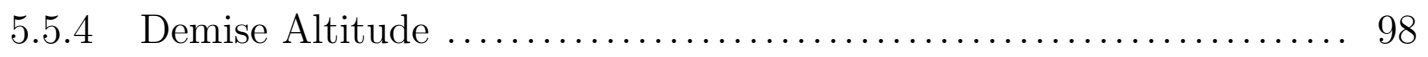

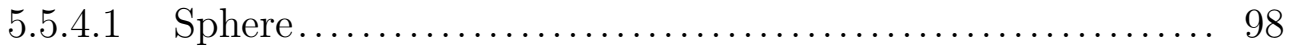




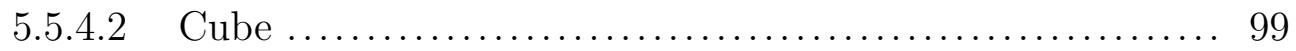

5.5.4.3 Cylinder...................................... 99

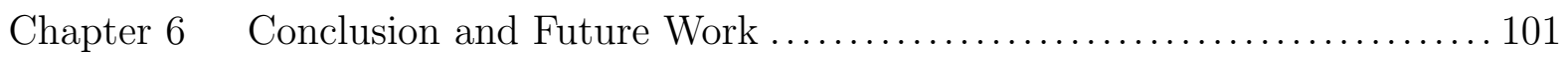

6.1 Conclusion ...................................................... 101

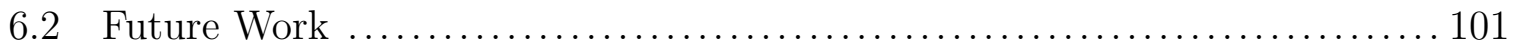

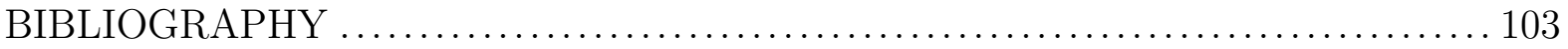

\section{APPENDICES}

Appendix A Nomenclature ................................................. 115

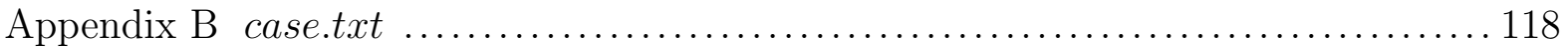

Appendix C Simulation Geometries ..................................... 119

Appendix D Materials Database .......................................... 120

Appendix E DAS Test Case Results ..................................... 122 


\section{LIST OF TABLES}

Table

Page

2.1 Comparison of selected reentry analysis tools

3.1 U.S. Standard Atmosphere Regions below $86 \mathrm{~km} \ldots \ldots \ldots \ldots \ldots \ldots \ldots \ldots$

3.2 Butcher Tableau for the Runge-Kutta-Fehlberg (4-5) Method ............. 20

3.3 Butcher Tableau for a Runge-Kutta 7-8 Method ..................... 21

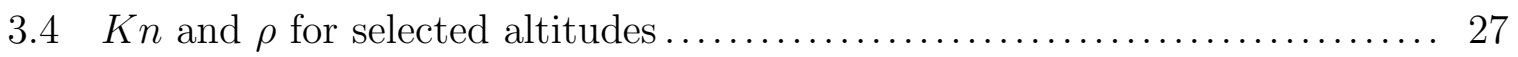

4.1 Material Properties of Selected Representative Materials................ 42

4.2 Initial Classical Orbital Elements............................... 42

4.3 Initial State Vector, Altitude, and Flight Path Angle.................. 43

4.4 Test Sphere Mass and Demise Internal Energy ..................... 44

4.5 Test Cube Mass and Demise Internal Energy $\ldots \ldots \ldots \ldots \ldots \ldots \ldots \ldots \ldots \ldots$

4.6 Test Cylinder Mass and Demise Internal Energy $\ldots \ldots \ldots \ldots \ldots \ldots \ldots \ldots \ldots .51$

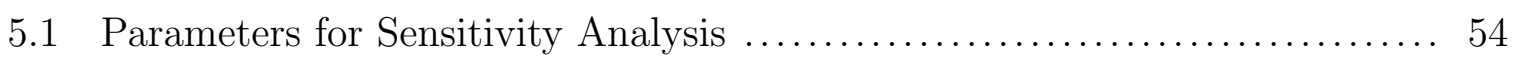




\section{LIST OF FIGURES}

Figure

1.1 Comparison of heat load profiles for a full re-entry and for a re-entry exposure starting at $75 \mathrm{~km}$...

2.1 Comparison of demise altitude predicted by ORSAT and SCARAB for each case

2.2 Comparison of impact mass predicted by ORSAT and SCARAB for each case

3.1 Atmospheric Properties Graphed from 0 to $85 \mathrm{~km}$ Altitude... 18

3.2 Variation of Normalized $c_{p}$ over Ram Surface of Sphere $\ldots \ldots \ldots \ldots \ldots \ldots \ldots$

3.3 Variation of Sphere Drag Coefficient with Altitude .................... 26

3.4 Variation of Drag Coefficient with Altitude......................... 28

3.5 Variation of Normalized Heat Flux over Ram Surface of Sphere.......... 29

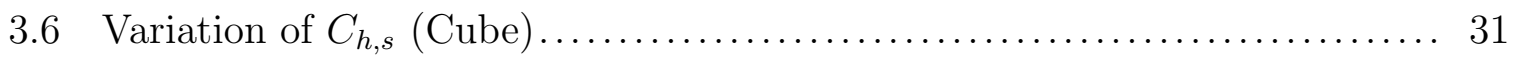

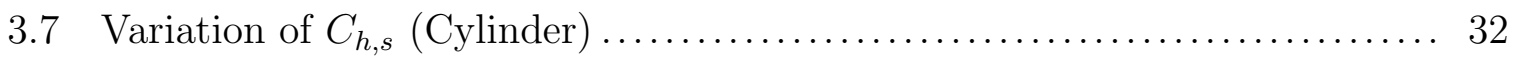

3.8 Software Architecture of OSTROM $\ldots \ldots \ldots \ldots \ldots \ldots \ldots \ldots \ldots \ldots \ldots \ldots \ldots \ldots$

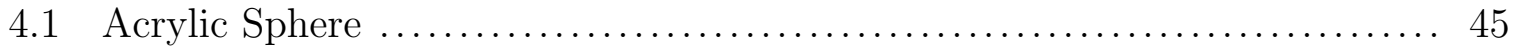

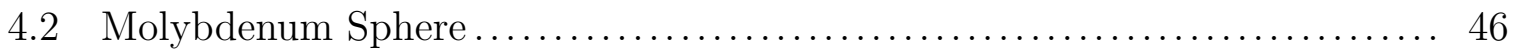

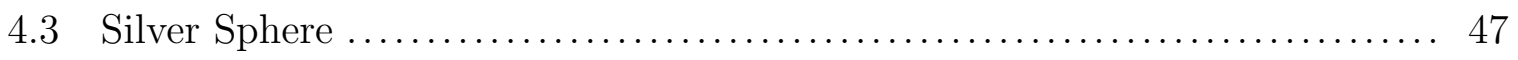

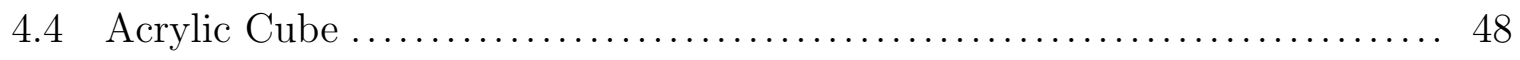

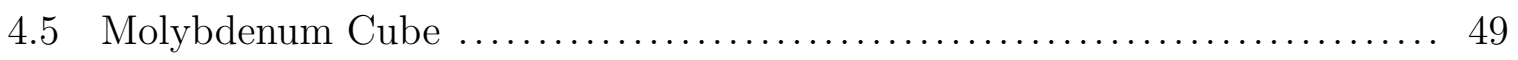




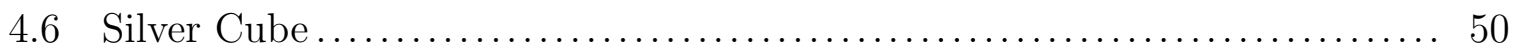

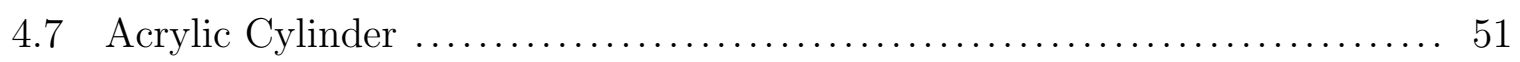

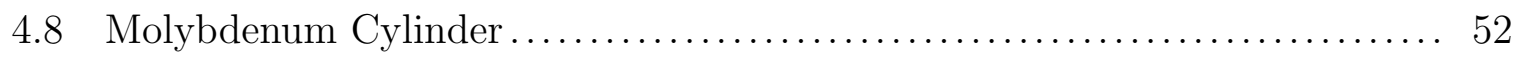

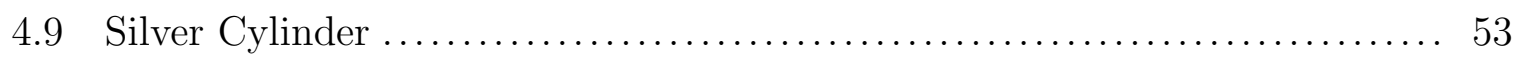

5.1 Downrange Distance vs. Initial Speed (Sphere) ….................. 55

5.2 Downrange Distance vs. Initial Speed (Cube) ..................... 56

5.3 Downrange Distance vs. Initial Speed (Cylinder) . . . . . . . . . . . . . . . 57

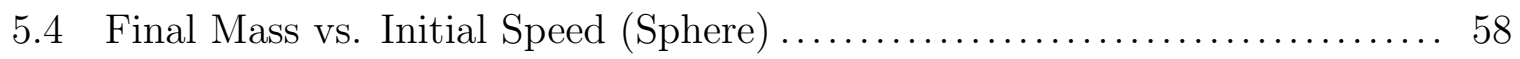

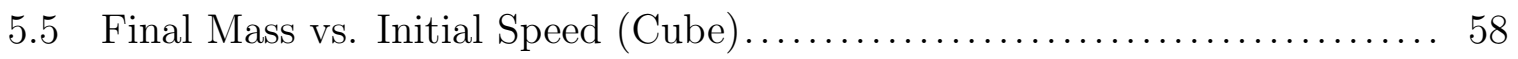

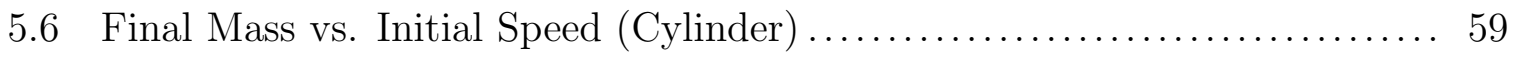

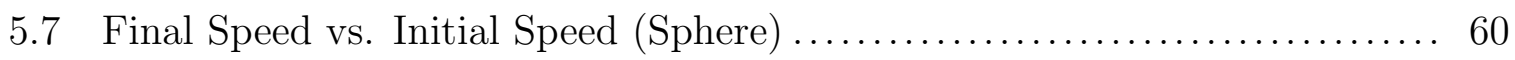

5.8 Final Speed vs. Initial Speed (Cube) $\ldots \ldots \ldots \ldots \ldots \ldots \ldots \ldots \ldots \ldots \ldots \ldots \ldots \ldots \ldots \ldots \ldots \ldots \ldots \ldots$

5.9 Final Speed vs. Initial Speed (Cylinder) …...................... 61

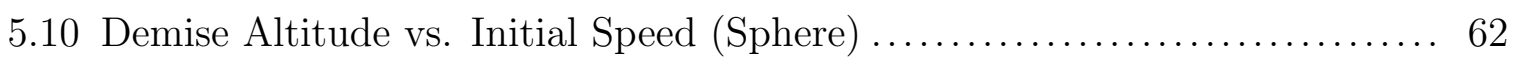

5.11 Demise Altitude vs. Initial Speed (Cube) $\ldots \ldots \ldots \ldots \ldots \ldots \ldots \ldots \ldots \ldots \ldots \ldots \ldots \ldots \ldots \ldots$

5.12 Demise Altitude vs. Initial Speed (Cylinder) ....................... 63

5.13 Downrange Distance vs. Initial Altitude (Sphere) …................ 65

5.14 Downrange Distance vs. Initial Altitude (Cube) ....................... 65

5.15 Downrange Distance vs. Initial Altitude (Cylinder) .................. 66

5.16 Final Mass vs. Initial Altitude (Sphere) …........................ 67

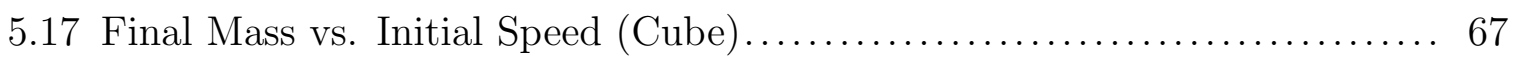

5.18 Final Mass vs. Initial Speed (Cylinder) ............................ 68

5.19 Final Speed vs. Initial Altitude (Sphere) $\ldots \ldots \ldots \ldots \ldots \ldots \ldots \ldots \ldots \ldots \ldots \ldots \ldots \ldots$

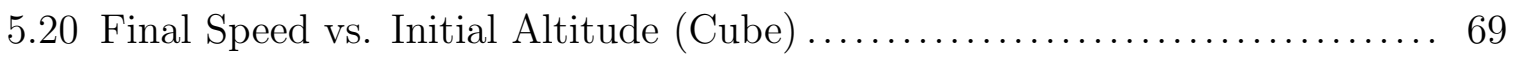

5.21 Final Speed vs. Initial Altitude (Cylinder) …................... 70

5.22 Demise Altitude vs. Initial Altitude (Sphere) …................... 71

5.23 Demise Altitude vs. Initial Altitude (Cube) ….................... 72 


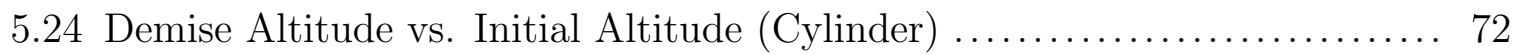

5.25 Downrange Distance vs. Temperature Scaling Factor (Sphere) ........... 74

5.26 Downrange Distance vs. Temperature Scaling Factor (Cube) ............ 74

5.27 Downrange Distance vs. Temperature Scaling Factor (Cylinder) …...... 75

5.28 Final Mass vs. Temperature Scaling Factor (Sphere) ................... 76

5.29 Final Mass vs. Temperature Scaling Factor (Cube) .................... 76

5.30 Final Mass vs. Temperature Scaling Factor (Cylinder) …............ 77

5.31 Final Speed vs. Temperature Scaling Factor (Sphere) $\ldots \ldots \ldots \ldots \ldots \ldots \ldots \ldots$

5.32 Final Speed vs. Temperature Scaling Factor (Cube) .................... 79

5.33 Final Speed vs. Temperature Scaling Factor (Cylinder) …............ 79

5.34 Demise Altitude vs. Temperature Scaling Factor (Sphere) .............. 80

5.35 Demise Altitude vs. Temperature Scaling Factor (Cube) ................ 81

5.36 Demise Altitude vs. Temperature Scaling Factor (Cylinder) ............. 82

5.37 Downrange Distance vs. Density Scaling Factor (Sphere) ............... 83

5.38 Downrange Distance vs. Density Scaling Factor (Cube) ................. 84

5.39 Downrange Distance vs. Density Scaling Factor (Cylinder) .............. 84

5.40 Final Mass vs. Density Scaling Factor (Sphere) ....................... 85

5.41 Final Mass vs. Density Scaling Factor (Cube) $\ldots \ldots \ldots \ldots \ldots \ldots \ldots \ldots \ldots \ldots . \ldots 6$

5.42 Final Mass vs. Density Scaling Factor (Cylinder) .................... 86

5.43 Final Speed vs. Density Scaling Factor (Sphere) .................... 87

5.44 Final Speed vs. Density Scaling Factor (Cube) $\ldots \ldots \ldots \ldots \ldots \ldots \ldots \ldots \ldots . \ldots 8$

5.45 Final Speed vs. Density Scaling Factor (Cylinder) .................... 89

5.46 Demise Altitude vs. Density Scaling Factor (Sphere) ................... 90

5.47 Demise Altitude vs. Density Scaling Factor (Cube) $\ldots \ldots \ldots \ldots \ldots \ldots \ldots \ldots . \ldots$

5.48 Demise Altitude vs. Density Scaling Factor (Cylinder) .................. 91

5.49 Downrange Distance vs. Initial Temperature (Sphere) .................. 92

5.50 Downrange Distance vs. Initial Temperature (Cube) ................... 93 
5.51 Downrange Distance vs. Initial Temperature (Cylinder) $\ldots \ldots \ldots \ldots \ldots \ldots \ldots . . \ldots 3$

5.52 Final Mass vs. Initial Temperature (Sphere) ........................ 94

5.53 Final Mass vs. Initial Temperature (Cube) .......................... 95

5.54 Final Mass vs. Initial Temperature (Cylinder) $\ldots \ldots \ldots \ldots \ldots \ldots \ldots \ldots \ldots . . \ldots 5$

5.55 Final Speed vs. Initial Temperature (Sphere) ..................... 96

5.56 Final Speed vs. Initial Temperature (Cube) $\ldots \ldots \ldots \ldots \ldots \ldots \ldots \ldots \ldots \ldots \ldots$

5.57 Final Speed vs. Initial Temperature (Cylinder) $\ldots \ldots \ldots \ldots \ldots \ldots \ldots \ldots \ldots . . \ldots 7$

5.58 Demise Altitude vs. Initial Temperature $($ Sphere) $\ldots \ldots \ldots \ldots \ldots \ldots \ldots \ldots . . \ldots 8$

5.59 Demise Altitude vs. Initial Temperature (Cube) ..................... 99

5.60 Demise Altitude vs. Initial Temperature (Cylinder) ................... 100 


\section{Chapter 1}

\section{Introduction}

\subsection{Purpose}

This thesis investigates the design and synthesis of an open-source spacecraft reentry analysis software package. Atmospheric reentry is a critical part of the post-mission spacecraft disposal phase, and the ability to ensure that a spacecraft will reenter in a safe manner- even in the event of uncontrolled reentry- is crucial for future missions. The code developed in this thesis is intended to be used early in the design process, in conjunction with the Design-for-Demise philosophy as detailed by Waswa[1]. The results generated by the code are compared with tests run in NASA's DAS program.

\subsection{Regulatory Requirements}

To prevent the further generation of space debris during and after the useful lifetime of a spacecraft, NASA, ESA, and other space agencies have set forth requirements which must be met for missions commissioned by those agencies. The standards NASA-STD-8719.14A Process for Limiting Orbital Debris[2], IADC-2002-01 IADC Space Debris Mitigation Guidelines[3], IADC-2004-03 Protection Manual[4], and Requirements on Space Debris Mitigation for ESA Projects[5] each detail methods of assessing the debris risk associated with a spacecraft design, as well as ways to mitigate this risk.

Allowing objects to reenter the atmosphere is one suggested method to reduce the debris in lower orbit. NASA-STD-8719.14A requires that any debris released by a spacecraft: (1) fully demise in the atmosphere; (2) in any case, strike the surface of the earth with no more than 15 Joules of kinetic energy, with an associated casualty risk of 1:10000, calculated based on ground track and longitude-averaged population density; or (3) in case of controlled reentry, impact the surface no closer than 370 kilometers from the shore of a foreign country, no closer than 50 kilometers from the shore of the USA, and not 
land on Antarctica[2]. The ESA document Requirements on Space Debris Mitigation for ESA Projects details similar requirements, including the suggested 1:10000 casualty risk, along with a 25-year lifetime for spacecraft and launchers in Low Earth Orbit (LEO), defined as any orbit under $2000 \mathrm{~km}$ altitude[5].

\subsection{Reentry Modeling}

There is no easy way to get experimental data on Earth for the flow conditions seen in atmospheric reentry, and doing tests with full satellites would be prohibitively expensive, so several programs have been written to simulate reentry. The most relevant to this thesis are NASA's DAS and ORSAT, and ESA's SCARAB packages. A detailed description of each of these can be found in their respective sections of Chapter 2 .

It was recognized as early as the 1960's that reentry prediction was a problem that would not be easily solved. The ability to successfully determine where and when a spacecraft would reenter was critical for the development of a manned space program.[6] There are, however, factors that make these predictions very difficult, perhaps the most important of which is the wide variability in atmospheric density from day to day.[7] The first attempts at numerically modeling reentry of space debris was undertaken by the US Air Force in the mid-1960's after parts of Sputnik IV reentered over Wisconsin in 1962.[8]

As it became clear that certain spacecraft components were surviving to impact the surface of the Earth, the idea of "design-for-demise", or choosing materials and construction in a fashion that ensures safety upon end-of-life, came into the front of many designers' minds.[1,9] The philosophy of designing a satellite to demise during reentry requires a way to accurately assess a design for survivability and risk.

In the 1990's and 2000's, space agencies NASA and ESA contracted the development of reentry analysis tools, resulting in the production of the DAS, ORSAT, and SCARAB codes. While software was developed for use by these government agencies, other research was being done at universities to analyze the risk associated with controlled and 
uncontrolled reentries of space vehicles.[10, 11, 12, 13, 14, 15, 16]

\subsection{Thesis Scope and Overview}

The programs listed in the previous section have the benefit of a long term of usage (and hence user familiarity), but are aging. In the case of DAS, some new computers cannot run the software directly: DAS was written for 16-bit architectures, and many new computers are 64-bit, requiring the use of a 32-bit virtual machine. NASA does not offer ORSAT for public use; nor has the ESA released SCARAB. None of the currently available reentry software applications are user-modifiable.

This thesis outlines the foundation for a general reentry object modeling toolkit similar to NASA's DAS, with the added benefit of being completely user-modifiable. If a user determines that one of the modules included in this package does not meet their needs, they are able to develop and use a new one of their own. For example, if a user required lower uncertainty in atmospheric conditions, they could write a model that includes latitude and longitude effects, or that uses solar flux updates from the internet, modify the base code, recompile, and have a better working custom solution.

\subsection{Assumptions}

\subsubsection{Attitude}

It will be assumed that the objects are at a constant attitude throughout the trajectory. This allows the removal of six differential equations from the simulation (going from 14 to 8)- three for angular position, three for angular velocity - which greatly reduces computational time. It is possible for a reentry object to have a constant attitude during its descent, but it is extremely unlikely for an uncontrolled reentry.

\subsubsection{Angle of Attack}

The angle of attack for each body is assumed to zero, meaning the trajectories are nonlifting (assuming the body is symmetric), and that the only forces acting upon the body 
are gravity (along the radius vector) and drag (along the velocity vector). Drag and gravity are the largest forces acting on a body at the altitudes this thesis' code simulates.

\subsubsection{Heat Transfer}

A lumped thermal mass (1-D heat transfer) model is implemented here, similar to the NASA reentry codes DAS and ORSAT. This model implies a rotating geometry, such that the heat transfer is equal on all surfaces of the object (and the object itself has no internal thermal gradients).

\subsubsection{Ablation}

Once the internal energy of a cell has reached the melting energy (see Eq. 1.1), it is assumed that the cell is removed from the body. The material, once ablated, is assumed to be immediately transported far from the body and is never redeposited. Modeling the redeposition of material would be more computationally expensive, but could be a useful addition in the future. Gross material removal by cracking or breaking off large chunks is not modeled here, as no internal stresses are calculated.

$$
e_{\text {ablation }}=c_{p} \cdot T_{\text {melt }}+h_{f}
$$

\subsubsection{Isentropic Flow}

A bow shock will develop slightly upstream of the blunt bodies modeled in this thesis, an area of high entropy gradient. Everywhere in the flow field far from the shock is assumed to have no entropy gradient, allowing for the use of the isentropic flow relations between static and stagnation conditions. Typically, this assumption is only used for freestream Mach numbers less than 5, since aerodynamic and viscous heating, dissociation, and ionization begin to change the static-to-stagnation ratios. Using the isentropic relations in the high Mach region $(M>5)$ will cause the stagnation temperature and pressure to be as much as 10\% higher than in reality.[17] The drag and heat flux on the reentry body will then be higher than expected, resulting in a lower speed on impact, and potentially 


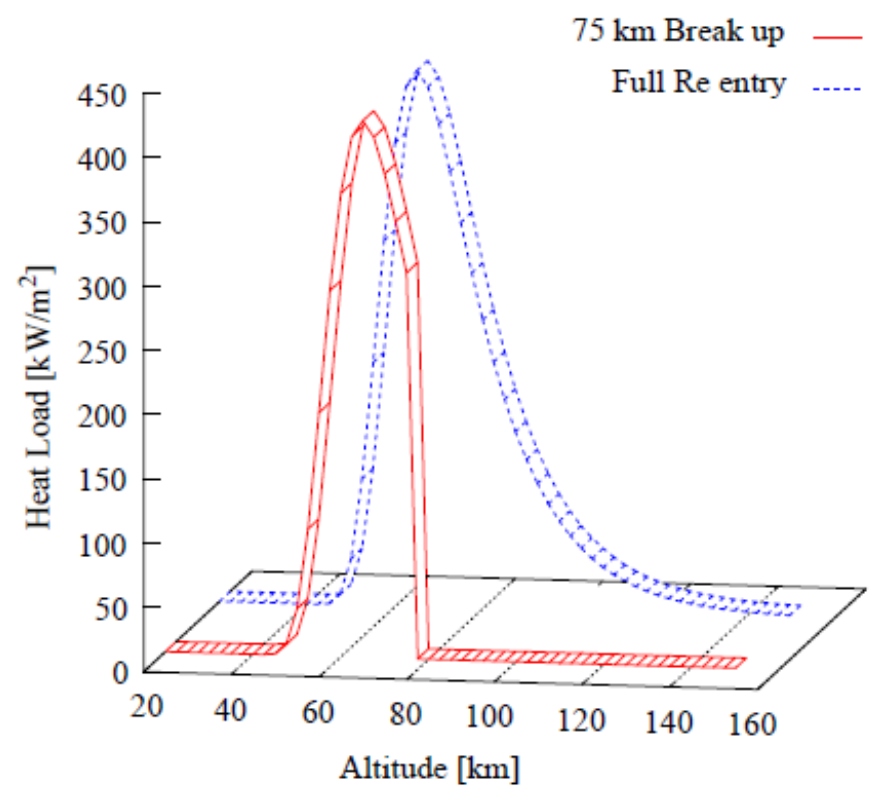

Figure 1.1: Comparison of heat load profiles for a full re-entry and for a re-entry exposure starting at $75 \mathrm{~km}$

showing that an object will demise when it would actually survive. The larger heat flux is acceptable, since the total heat load experienced during a reentry is much higher than only the heat load from $78 \mathrm{~km}$ and lower, seen by comparing the area under the curves in Figure 1.1(Figure 2 from [18]).

\subsubsection{Calorically Perfect Gas}

The calorically perfect gas (CPG) assumption allows the state equation seen in Equation 1.2, where $p$ is pressure, $\rho$ is density, $R$ is the universal gas constant, $T$ is temperature, and $M$ is the molar mass of the gas (or the weighted average of the constituents' molar masses in a mixture). The isentropic flow relations used in this thesis require a constant ratio of specific heats $(\gamma)$ - this assumption is valid at moderate temperatures (up to 600K) and pressures (up to $50 \mathrm{MPa}$ ). Above these temperatures and pressures, a compressibility factor, $Z$, can be used to adjust the accuracy of the ideal equation of state (see Equation $1.3)[19]$.

The difference between using the CPG model and a thermally perfect gas (where the 
specific heats are allowed to vary, but Equations 1.2 and 1.3 are still used) is around $6 \%$ (in stagnation-point pressure and temperature) at a Mach number of 10[17]. It was also noted in [17] that the isentropic flow equations are largely insensitive to changes in $\gamma$ at Mach numbers of 10 and higher.

$$
\begin{aligned}
& p=\rho \frac{R^{*}}{M} T \\
& p=Z \rho \frac{R^{*}}{M} T
\end{aligned}
$$




\section{Chapter 2}

\section{Previous Work}

\subsection{Reentry Analysis Tools}

Several attempts have been made to model the on-orbit and surface effects of spacecraft in decaying orbits by public and private entities, including NASA and ESA. This section details the reentry survivability analysis software packages DAS, ORSAT, and SCARAB, and discusses the differences between them.

\subsubsection{Debris Assessment Software (DAS)}

DAS was first developed in the 1990's at the NASA Johnson Space Center to aid in assessing orbital debris risk for NASA programs [20, 21]. DAS is described as "a conservative, easy to use tool" [22], and can be used to predict the effects of the spacecraft on the orbital debris environment while on-orbit and during reentry. A full reentry analysis can be performed in DAS, from building a model of the spacecraft from simple shapes (spheres, boxes, cylinders, and flat panels)[23] to defining the orbit parameters, and finally, assessing compliance with the NASA requirements discussed in Section 1.2.

DAS is publicly available for download from the NASA Orbital Debris Program Office website, and has modest requirements for installation and use. Version 2.0 of DAS was released in 2008, and included a GUI for the first time, affording easier access to less savvy users[24]. The structure of DAS can be described as the following parts: the Mission Editor, Requirement Assessment, Material Database, and Science and Engineering Utilities. [1, 23]

\subsubsection{Mission Editor}

Through the Mission Editor, the user can define a satellite, rocket body, or other debris related to the mission for orbital debris requirement assessments. Some of the properties 
that can be defined include launch year, mission duration, components of the mission, and certain orbital parameters, like perigee altitude, apogee altitude, inclination, RAAN, argument of perigee, initial mass, and final mass. [23]

\subsubsection{Requirement Assessment}

This utility contains supported requirements from NASA-STD-8719.14A [2]. Each requirement is checked against the data entered in the Mission Editor for compliance, then the status is presented.

\subsubsection{Material Database}

The material database included in DAS is reproduced in Appendix D. In addition to the 77 materials included by default, users can create and edit materials with different properties (specific heat capacity, heat of fusion, melting temperature, and density). Specific heat, heat of fusion, and melting temperature are used during reentry analysis; density is used to ensure the size and mass of components are consistent.[23]

\subsubsection{Science and Engineering Utilities}

This module is possibly the most important of DAS: it analyzes reentry survivability and probability of on-orbit collision, and calculates $\Delta V$ required for a post-mission orbital maneuver and $\Delta V$ required for a general orbit-to-orbit transfer. For each subcomponent of a spacecraft assembly, DAS can calculate demise altitude, impact kinetic energy (crucial for assessing compliance with NASA Standard 8719.14), and total debris casualty

area, which can be calculated using Equation 2.1, where $A_{i}$ is the area of the $i^{\text {th }}$ object surviving reentry and 0.6 is the square root of the average area of a person standing, seen from above.[23] In addition to the materials database discussed above, DAS uses solar flux (F10.7) tables retrieved from the NASA Orbital Debris Office at Johnson Space 
Center, which are updated approximately every six months.

$$
D_{A}=\sum_{i=1}^{N}\left(0.6+\sqrt{A_{i}}\right)^{2}
$$

\subsubsection{Object Reentry Survival Analysis Tool (ORSAT)}

ORSAT's latest version is 6.0, released in January 2006 for use by NASA personnel at Johnson Space Center.[25] Similar to DAS, ORSAT was developed by NASA as a destructive reentry analysis tool, although with higher fidelity. The casualty risk is estimated using debris casualty area, like DAS, but the method of determining the casualty area uses reentry year and orbital inclination in addition to the parameters DAS uses (shape, size, and motion assumptions).[1,26]

Reentry trajectories are computed using a 3-DOF model, using a Runge-Kutta 45 method (described in Section 3.2.1.1). Heat transfer is calculated using a lumped thermal mass model, as does DAS.[18, 23] The lumped thermal mass model describes a component as a single mass that gains and loses heat isotropically; spheres and cylinders can use a 1-D heat conduction model, which allows for modeling of partial ablation of a component. Material properties in ORSAT are temperature-dependent, unlike the static values used in DAS.[18] Spacecraft breakup is assumed to occur at $78 \mathrm{~km}$ altitude, after which each component is tracked until it demises or impacts the surface.

\subsubsection{Spacecraft Atmospheric Reentry \& Aerothermal Breakup (SCARAB)}

Unlike ORSAT and DAS, SCARAB was developed for ESA by a group of private contractors led by HTG (Hypersonic Technology Göttingen).[27] In the rarefied hypersonic

flow regime $(\mathrm{M}>6) \mathrm{SCARAB}$ uses a 6-DOF model, allowing for modeling of tumbling reentries at higher altitudes. For the continuum regime $(\mathrm{M}<6)$, a 3-DOF model is used, as rotation is likely to be damped out by atmospheric drag.[28]

In the transitional flow regime, heat transfer rate is calculated using the a bridging 
function of Stanton numbers, seen in Equation 2.2, where $S t_{c}$ is the continuum Stanton number and $S t_{F M}$ is the free molecular Stanton number.[27]

$$
S t=\frac{S t_{c}}{\sqrt{1+\left(\frac{S t_{c}}{S t_{F M}}\right)^{2}}}
$$

\subsection{Comparison of DAS, ORSAT, and SCARAB}

As opposed to ORSAT's and DAS's "object-oriented" method, SCARAB uses a "satelliteoriented" approach; this approach focuses on the overall spacecraft architecture and the links between components, as opposed to focusing on the components themselves.[26] The limitation of the survivability analysis to altitudes $75-85 \mathrm{~km}$ and lower in DAS and ORSAT dramatically reduces the heat load experienced by the spacecraft, causing the mass expected to impact the Earth to be greater than predicted by SCARAB, which starts its reentry analysis at altitudes over $150 \mathrm{~km}$.

SCARAB uses a full 6-DOF model for upper altitudes, allowing for modeling of spinning and tumbling of general shapes as well as translation of the object; DAS does not model tumbling for any object, and ORSAT can only model certain tumbling modes for certain shapes.[18] Since DAS and ORSAT do not simulate the breakup of the spacecraft itself, they do not include calculations of internal stresses like SCARAB does. Ultimate stress values in SCARAB vary with temperature, along with other 12 material properties like specific heat, thermal conductivity, and emissivity[29]; ORSAT uses temperature dependent specific heats and emissivity, among other quantities, and the material properties used in DAS' analyses are constant with temperature.

Orbital perturbations modeled by DAS and ORSAT include atmospheric drag, solar and lunar third-body gravity, solar radiation pressure (SRP), Earth shadow from SRP, and Earth oblateness. SCARAB accounts for these perturbations, as well as shear stress from the fluid flow (inducing rotation of the spacecraft), and the potential for simulating 
thrust pulses in SCARAB 3.[27]

NASA suggests that DAS be used first to analyze the reentry survival risk of a spacecraft, and to request an analysis of the spacecraft in ORSAT if the DAS requirement assessment module does not mark success.[18] Since DAS does not model partial melting or destruction of objects like ORSAT does, and starts its analysis at a lower altitude than SCARAB does, it gives a more conservative estimate of the casualty risk posed by a spacecraft. However, DAS and ORSAT both allow for easier preparation of a spacecraft model than SCARAB, which requires all connections between components to be modeled.[27]

The results achieved using a full destructive reentry code like SCARAB can be more realistic than those from ORSAT or DAS, with all other parameters being equal, since the analysis performed by SCARAB includes greater detail in heat transfer and 3-D modeling, as well as permitting spinning and tumbling. Figure 1.1 (Figure 2 from [18]) demonstrates how the total heat load experienced by a reentry object differs for simulations that start at the assumed breakup altitude of $75 \mathrm{~km}$ and those that perform a full reentry. When simple geometries like boxes and spheres were compared between ORSAT and SCARAB, it was found the two codes were in close agreement, as seen in Figures 2.1 and 2.2(Figures 1 and 2 from [28], respectively).

Table 2.1 lists the four reentry codes discussed in this thesis along with the type of aerothermal and heat transfer models and the typical initial altitude for a simulation. DAS, ORSAT, and SCARAB all use analytical models for heating and aerodynamic forces for both the free-molecular and continuum flow regimes, with some bridging functions to describe the transition between the two (ones based on Knudsen number for drag force, and ones based on Stanton number for aero-heating)[27]. The use of a lumped thermal mass model by both DAS and OSTROM means it is highly unlikely that they will be able to model partial ablation of a reentry body, since once the object reaches 


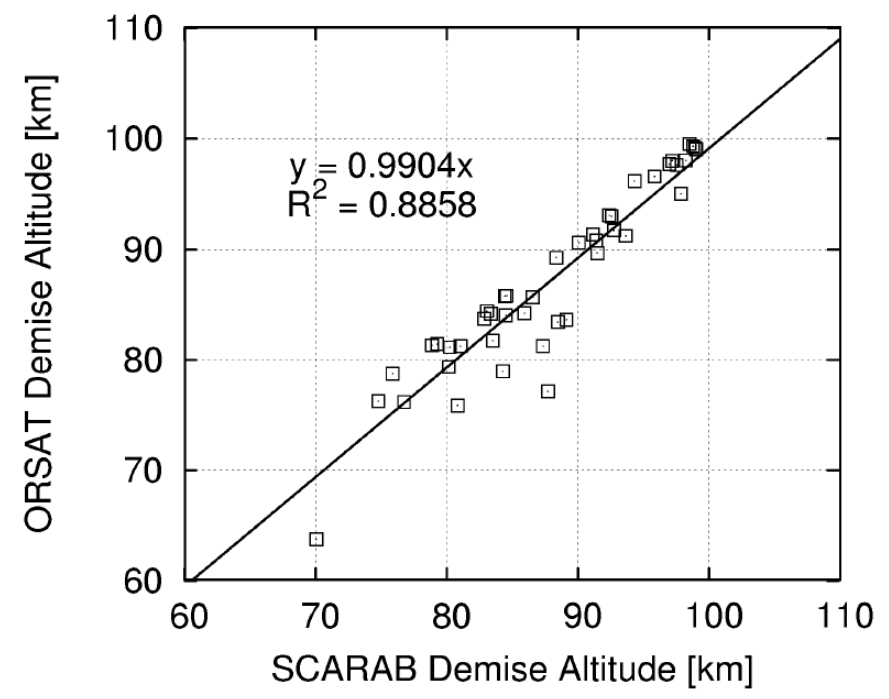

Figure 2.1: Comparison of demise altitude predicted by ORSAT and SCARAB for each case

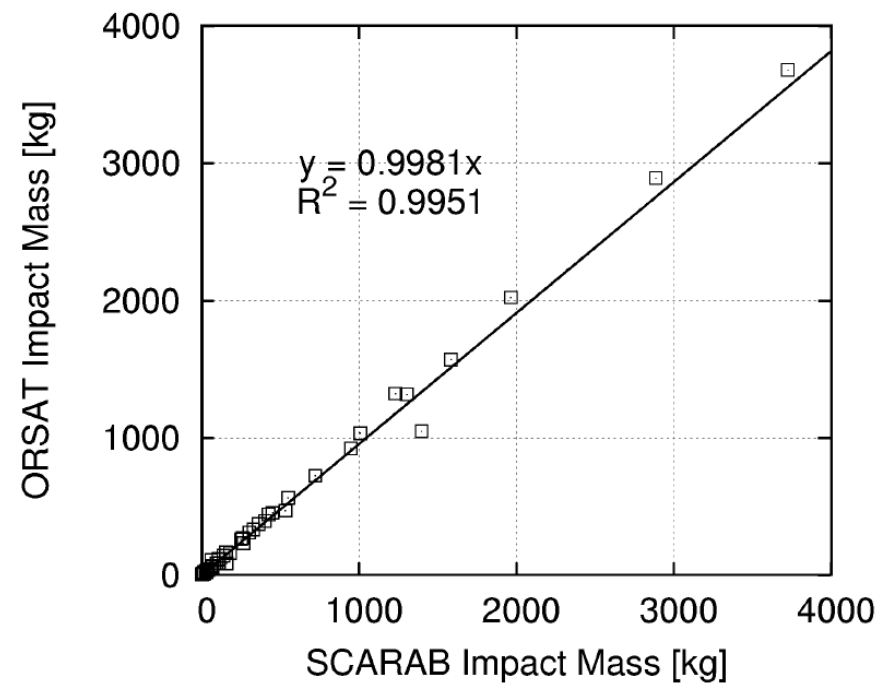

Figure 2.2: Comparison of impact mass predicted by ORSAT and SCARAB for each case 


\section{Table 2.1: Comparison of selected reentry analysis tools}

\begin{tabular}{|c|c|c|c|}
\hline & Aerothermal & Heat Transfer & Initial Altitude $(\mathrm{km})$ \\
\hline DAS & FM and Continuum & Lumped Thermal Mass & 78 \\
ORSAT & FM and Continuum & 1-D Conduction & 78 \\
SCARAB & FM and Continuum & 1-D Conduction & 122 \\
OSTROM & FM and Continuum & Lumped Thermal Mass & Configurable \\
\hline
\end{tabular}

its maximum temperature, any heat transfer after that is pure mass loss (governed by Equation 2.3). DAS and ORSAT begin their reentry calculations at an altitude of $78 \mathrm{~km}$, after the assumed breakup of the spacecraft; SCARAB, being a fully-destructive code capable of modeling the breakup, begins at a higher altitude; OSTROM can begin the simulation at any user-specified altitude, but is used at $78 \mathrm{~km}$ for this thesis' test cases for computational time and comparability of data.

$$
\left.\dot{m}\right|_{T=T_{\max }}=-\frac{\dot{Q}}{h_{f}}
$$




\section{Chapter 3}

\section{Models}

\subsection{Atmosphere}

The choice of atmospheric model is very important for reentry trajectory prediction uncertainty due to density error has one of the largest impacts on downrange distance and heating rates. The GRAM, MSISE, and U.S. Standard Atmosphere models were considered for inclusion in this thesis. Owing to its simplicity and accuracy at lower altitudes, the 1976 U.S. Standard Atmosphere[30] was selected.

\subsubsection{Global Reference Atmospheric Model (GRAM)}

Scientists at NASA originally developed the GRAM in the 1970's as an engineering tool for the Space Shuttle program. This model was different from the prior standard atmospheres in that it predicted the normal properties (temperature, density, and pressure) as well as accounting for changes not only in altitude, but in latitude, longitude, and time of year.[31] The GRAM has been updated several times: in 1980, 1990, 1995, 1999, 2007, and 2010. These updates added random atmospheric perturbations[32, 33], improved wind and turbulence predictions[34, 35], modified the temperature, pressure, and density values based on newer data[36], expanded the coverage of the model worldwide, and increased the vertical resolution[37].

One significant benefit of using GRAM is that it outputs all the data required for any analysis, including number densities of all constituent gases. However, the increased accuracy of GRAM comes with several drawbacks, which led to the choice not to use it in this thesis: first, GRAM is very complex, with many inputs required to perform any single calculation of atmospheric properties, which at this stage, are not known to any potential user of this software; second, the computational expense of the model is higher than that of the U.S. Standard Atmosphere; and last, most of the important altitude 
range of this model lies above the areas of interest for this thesis, mainly below $90 \mathrm{~km}$.

\subsubsection{Mass Spectrometer and Incoherent Scatter Radar (MSISE)}

The first MSIS atmospheric model was developed in the mid 1970's by an international team from NASA, MIT, Canada, and France as an extension of the earlier Jacchia 1971 model[38], which had been primarily derived from satellite drag data. MSIS used onorbit mass spectrometer data and ground station incoherent scatter measurements to model thermospheric gas composition, temperature, and density with varying: altitude, latitude, longitude, $F_{10.7}$ value (a measure of solar activity), season, and even time of day.[39]

Adjustments were made to the MSIS in 1983 to add atomic oxygen density as an output, modeling the effects of a "daily magnetic index" [40], incorporating data collected by another ground station (and more data from the prior sources from the intervening decade). In 1986, new data had been collected from Dynamics Explorer I and II, a pair of polar satellites, that drastically improved the representation of the atmosphere at higher latitudes[41]. A large change occurred in 1990, when the title was changed to the MSISE, and the model was extended into the middle and lower regions of the atmosphere using harmonic functions and Fourier series approximations to empirical data[42]. The current model is the NRLMSISE-00, released in 2001 by the U.S. Naval Research Laboratory (NRL), and incorporates new drag and radar data to improve its estimation of atmospheric density at higher altitudes (500 km and above)[43]. As with GRAM, this model is far more complex than the U.S. Standard Atmosphere, especially at the lower altitudes, and was not selected for implementation in this thesis.

\subsubsection{U.S. Standard Atmosphere}

The U.S. Standard Atmosphere (USSTDATM) was originally developed by NASA, NOAA, and the USAF in the 1950's, and was updated in 1976. It describes a gravitational equilibrium atmosphere from the surface to 1000 kilometers altitude. Below 32 kilometers, it 
is identical to the ICAO International Standard Atmosphere. This atmospheric model is by far the simplest, allowing for easier programming and code verification. USSTDATM is broken into seven regions below 86 kilometers altitude, with static temperature, pressure, and temperature lapse rate (the amount it decreases per unit of altitude) defined at the lower boundary of each region, summarized in Table 3.1.

\begin{tabular}{c|c|c|c|c}
\multicolumn{4}{c}{ Table 3.1: U.S. Standard Atmosphere Regions below $86 \mathbf{~ k m}$} \\
Region & Altitude (m) & Temperature (K) & Pressure (Pa) & Lapse Rate $\left(\frac{K}{m}\right)$ \\
\hline 0 & 0 & 288.15 & 101325 & -0.0065 \\
1 & 11000 & 216.65 & 22632.1 & 0.0 \\
2 & 20000 & 216.65 & 5474.89 & 0.001 \\
3 & 32000 & 228.65 & 868.019 & 0.0028 \\
4 & 47000 & 270.65 & 110.906 & 0.0 \\
5 & 51000 & 270.65 & 66.9389 & -0.0028 \\
6 & 71000 & 214.65 & 3.95642 & -0.02
\end{tabular}

The manual published by NASA, NOAA, and the USAF also details the equations needed to use these data to model these properties at all altitudes in the regions, not just at the boundaries. Temperature is calculated with a simple linear formula, 3.1, where $h$ is the altitude (in $m$ ) in question, $T_{0, i}$ is the temperature at the nearest lower boundary (in $K$ ), $L_{i}$ is the lapse rate for that region (in $K / m$ ), and $H_{i}$ is the altitude of the nearest lower boundary (in $m$ ).

As is seen in Table 3.1, Regions 2 and 5 have no temperature lapse with altitude, so the isothermal equation for pressure must be used: 3.2. For all other regions, a more complex equation is used, which accounts for the change in temperature within a region: 3.3. Similarly, the USSTDATM model prescribes equations for calculating the density in isothermal (Equation 3.4) and anisothermal regions (Equation 3.5). All equations below 
are reproduced from [30].

$$
\begin{aligned}
& T(h)=T_{0, i}+L_{i} *\left(h-H_{i}\right) \\
& p(h)=p_{0, i} \cdot \exp \left[\frac{-g_{0} \cdot M \cdot\left(h-H_{i}\right)}{R^{\star} \cdot T_{0, i}}\right] \\
& p(h)=p_{0, i} \cdot\left[\frac{T_{0, i}}{T_{0, i}+L_{i} \cdot\left(h-H_{i}\right)}\right]^{\frac{g_{0} \cdot M}{R^{\star} \cdot L_{i}}} \\
& \rho(h)=\rho_{0, i} \cdot \exp \left[\frac{-g_{0} \cdot M \cdot\left(h-H_{i}\right)}{R^{\star} \cdot T_{0, i}}\right] \\
& \rho(h)=\rho_{0, i} \cdot\left[\frac{T_{0, i}+L_{i} \cdot\left(h-H_{i}\right)}{T_{0, i}}\right]^{\left(1+\frac{g_{0} \cdot M}{R^{\star} \cdot L_{i}}\right)}
\end{aligned}
$$

From these equations, a function was written that calculates the atmospheric temperature and density. To show graphically the trends in density and temperature with altitude, a script was made to call the function repeatedly and plot the results, seen in Figure 3.1. The uppermost point in the graphs is at 84,852 meters, the upper bound for Region 7. Above this altitude, the governing equations are significantly different from and more complex than the ones listed above and are not included in this thesis. 

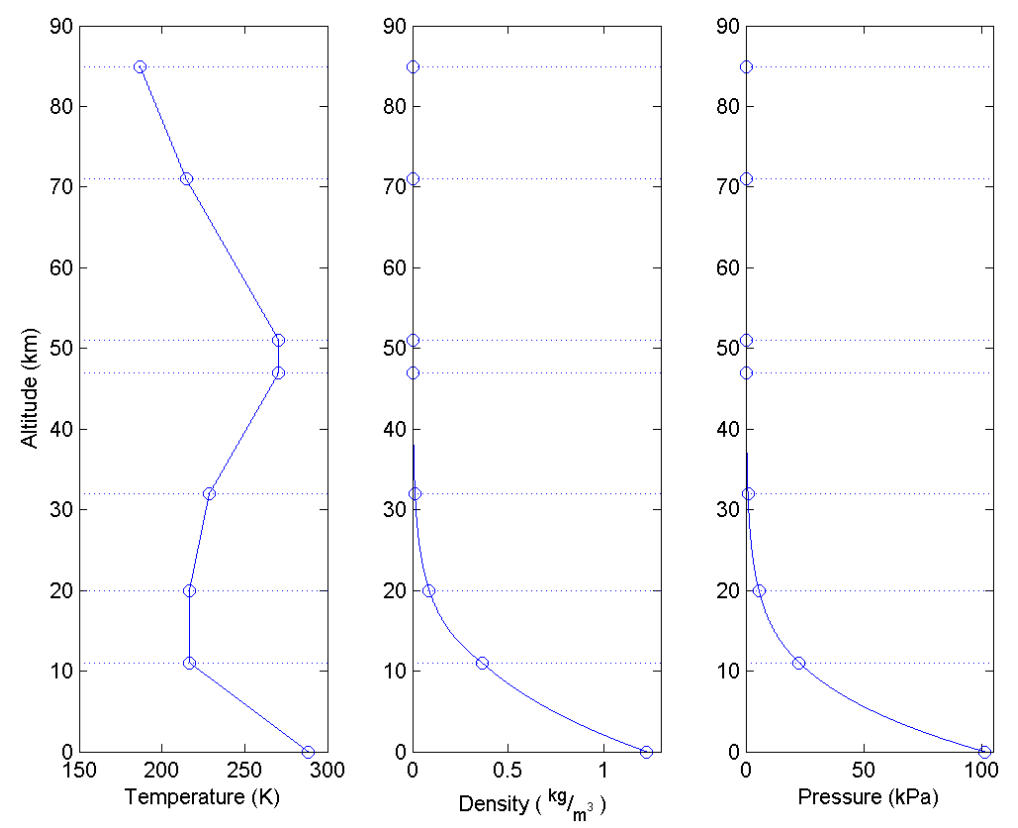

\section{Figure 3.1: Atmospheric Properties Graphed from 0 to $85 \mathrm{~km}$ Altitude}

A table of temperature, pressure, and density values from 0-1000 km (in 10-meter increments) was generated using the formulas listed above (and in [30]), then included in the code itself. This increased the size of the executable by about $5 \mathrm{MB}$, but also drastically reduced the time taken to calculate these values (requiring only a linear interpolation between table values).

\subsection{Orbit Propagation}

\subsubsection{Runge-Kutta Methods}

Integrating the equations of motion for a system that has rapidly changing quantities (like mass, velocity, and position) while ensuring low error can be difficult to do with lowerorder numerical methods. Three methods that were considered for this thesis include the Runge-Kutta-Fehlberg 4(5) method (RKF45)[44], a Runge-Kutta 7(8) method (RK78) detailed by Fehlberg in [45], and a Runge-Kutta 1(8) method created for this thesis, which substitutes the $8^{\text {th }}$ order corrector of RK78 into the first order Euler method. Equations 
3.6 and 3.7 are general for any order Runge-Kutta method and are reproduced from [45]. The coefficients $\alpha_{k}, \beta_{k \lambda}$, and $c_{k}$ are determined by solving a system of linear and nonlinear equations, the algorithm of which is found in [46].

$$
\begin{aligned}
& f_{0}=f\left(x_{0}, y_{0}\right) \\
& f_{\kappa}=f\left(x_{0}+\alpha_{\kappa} h, y_{0}+h \sum_{\lambda=0}^{\kappa-1} \beta_{\kappa \lambda} f_{\lambda}\right)
\end{aligned}
$$

\subsubsection{RKF45}

RKF45 (also known as "the" Runge-Kutta method) is a 4th-order numerical integration scheme with a 5th-order corrector. This corrector allows the solver to increase the order of the error locally and globally by one with the addition of only one more function evaluation per step. The combination of the two differently-ordered formulae also allows for step size control based on the error between the two methods. The 4th-order steps are defined by Equations 3.6, 3.7, and 3.8; the 5th-order corrector steps are defined by Equations 3.6, 3.7, and 3.9, where $\alpha_{k}, \beta_{k \lambda}$, and $c_{k}$ are defined in Table 3.2 (Table III from $[44])$.

$$
\begin{array}{r}
{[! h] y=y_{0}+h \sum_{\kappa=0}^{4} c_{\kappa} f_{\kappa}+\mathcal{O}\left(h^{4}\right)} \\
\hat{y}=y_{0}+h \sum_{\kappa=0}^{5} \hat{c}_{\kappa} f_{\kappa}+\mathcal{O}\left(h^{5}\right)
\end{array}
$$


Table 3.2: Butcher Tableau for the Runge-Kutta-Fehlberg (4-5) Method

\begin{tabular}{|c|c|c|c|c|c|c|c|c|}
\hline \multicolumn{7}{|c|}{} & \multicolumn{7}{|c|}{$\beta_{\kappa \lambda}$} & \multicolumn{2}{l|}{} \\
\hline$\kappa \backslash^{\lambda}$ & $\alpha_{\kappa}$ & 0 & 1 & 2 & 3 & 4 & $c_{k}$ & $\hat{c}_{\kappa}$ \\
\hline 0 & 0 & 0 & & & & & $\frac{25}{216}$ & $\frac{16}{135}$ \\
1 & $\frac{1}{4}$ & $\frac{1}{4}$ & & & & & \multicolumn{2}{|c|}{0} \\
2 & $\frac{3}{8}$ & $\frac{3}{32}$ & $\frac{9}{32}$ & & & & $\frac{1408}{2565}$ & $\frac{6656}{12825}$ \\
3 & $\frac{12}{13}$ & $\frac{1932}{2197}$ & $-\frac{7200}{2197}$ & $\frac{7296}{2197}$ & & & $\frac{2197}{4104}$ & $\frac{28561}{56430}$ \\
4 & 1 & $\frac{439}{216}$ & -8 & $\frac{3680}{513}$ & $-\frac{845}{4104}$ & & $\frac{1}{5}$ & $-\frac{9}{50}$ \\
\hline 5 & $\frac{1}{2}$ & $-\frac{8}{27}$ & 2 & $-\frac{3544}{2565}$ & $\frac{1859}{4104}$ & $-\frac{11}{40}$ & & $\frac{2}{55}$ \\
\hline
\end{tabular}

\subsubsection{RK78}

Similar to the RKF45 method, RK78 uses two Runge-Kutta formulae of different orders together to improve the estimate of the solution. Unlike RKF45, RK78 requires 2 more function evaluations per step for the higher-order corrector. The 7th-order predictor step is defined by Equations 3.6, 3.7, and 3.10; the 8th-order corrector is defined by Equations 3.6, 3.7, and 3.11, where $\alpha_{k}, \beta_{k \lambda}$, and $c_{k}$ are defined in Table 3.3 (Table X from [45]). The RK78 method was implemented and passed validation tests, but caused the simulation to diverge after several thousand time steps, so the RK18 method was ultimately chosen.

$$
\begin{aligned}
& y=y_{0}+h \sum_{\kappa=0}^{10} c_{\kappa} f_{\kappa}+\mathcal{O}\left(h^{8}\right) \\
& \hat{y}=y_{0}+h \sum_{\kappa=0}^{12} \hat{c}_{\kappa} f_{\kappa}+\mathcal{O}\left(h^{9}\right)
\end{aligned}
$$


Table 3.3: Butcher Tableau for a Runge-Kutta 7-8 Method

\begin{tabular}{|c|c|c|c|c|c|c|c|c|c|c|c|c|c|c|c|}
\hline \multicolumn{14}{|c|}{$\beta_{\kappa \lambda}$} & \multirow[b]{2}{*}{$c_{k}$} & \multirow[b]{2}{*}{$\hat{c}_{\kappa}$} \\
\hline$\kappa \backslash^{\lambda}$ & $\alpha_{\kappa}$ & 0 & 1 & 2 & 3 & 4 & 5 & 6 & 7 & 8 & 9 & 10 & 11 & & \\
\hline 0 & 0 & 0 & & & & & & & & & & & & $\frac{41}{840}$ & 0 \\
\hline 1 & $\frac{2}{27}$ & $\frac{2}{27}$ & & & & & & & & & & & & & \\
\hline 2 & $\frac{1}{9}$ & $\frac{1}{36}$ & $\frac{1}{12}$ & & & & & & & & & & & & \\
\hline 3 & $\frac{1}{6}$ & $\frac{1}{24}$ & 0 & $\frac{1}{8}$ & & & & & & & & & & & \\
\hline 4 & $\frac{5}{12}$ & $\frac{5}{12}$ & 0 & $-\frac{25}{16}$ & $\frac{25}{16}$ & & & & & & & & & & \\
\hline 5 & $\frac{1}{2}$ & $\frac{1}{20}$ & 0 & 0 & $\frac{1}{4}$ & $\frac{1}{5}$ & & & & & & & & & \\
\hline 6 & $\frac{5}{6}$ & $-\frac{25}{108}$ & 0 & 0 & $\frac{125}{108}$ & $-\frac{65}{27}$ & $\frac{125}{54}$ & & & & & & & & \\
\hline 7 & $\frac{1}{6}$ & $\frac{31}{300}$ & 0 & 0 & 0 & $\frac{61}{225}$ & $-\frac{2}{9}$ & $\frac{13}{900}$ & & & & & & & \\
\hline 8 & $\frac{2}{3}$ & 2 & 0 & 0 & $-\frac{53}{6}$ & $\frac{704}{45}$ & $-\frac{107}{9}$ & $\frac{67}{90}$ & 3 & & & & & & \\
\hline 9 & $\frac{1}{3}$ & $-\frac{91}{108}$ & 0 & 0 & $\frac{23}{108}$ & $-\frac{976}{135}$ & $\frac{311}{54}$ & $-\frac{19}{60}$ & $\frac{17}{6}$ & $-\frac{1}{12}$ & & & & & \\
\hline 10 & 1 & $\frac{2383}{4100}$ & 0 & 0 & $-\frac{341}{164}$ & $\frac{4496}{1025}$ & $-\frac{301}{82}$ & $\frac{2133}{4100}$ & $\frac{45}{82}$ & $\frac{45}{164}$ & $\frac{18}{41}$ & & & $\frac{41}{840}$ & 0 \\
\hline 11 & 0 & $\frac{3}{205}$ & 0 & 0 & 0 & 0 & $-\frac{6}{41}$ & $-\frac{3}{205}$ & $-\frac{3}{41}$ & $\frac{3}{41}$ & $\frac{6}{41}$ & 0 & & & $\frac{41}{840}$ \\
\hline 12 & 1 & $-\frac{1777}{4100}$ & 0 & 0 & $-\frac{341}{164}$ & $\frac{4496}{1025}$ & $-\frac{289}{82}$ & $\frac{2193}{4100}$ & $\frac{51}{82}$ & $\frac{33}{164}$ & $\frac{12}{41}$ & 0 & 1 & & $\frac{41}{840}$ \\
\hline
\end{tabular}

\subsubsection{RK18}

The RK18 method used in this thesis' code was developed as a low-error solver for systems of stiff ordinary differential equations, using the 8th-order corrector detailed in Section 3.2.1.2 with a 1st-order predictor (the Euler method), specified in Equation 3.12. The low-order predictor forces the solver to use much smaller timesteps, which can allow for greater numerical stability (as long as the error tolerances are small).

$$
\vec{y}_{i+1}=\vec{y}_{i}+h f\left(t_{i}, \vec{y}_{i}\right)+\mathcal{O}\left(h^{2}\right)
$$




\subsubsection{Step Size Control}

Step size can be changed in a few different ways: the first, and simplest, is to simply double or halve the step size in response to meeting or exceeding the error tolerances (Eq. 3.13). Second, a technique called Richardson extrapolation can be used to increase the order of the error by repeatedly canceling out successive terms of the Taylor series expansion $[47,48]$. Third, an expression relating the order of accuracy of the method and the current truncation error to the step size can be used (see Eq. 3.14, where $q$ is the order of the solver and tol is the error tolerance) [49, 50, 47]. A modification (see Eq. 3.15) has been suggested to Eq. 3.14 as a way to implement a "safety factor" on the step size: multiplying the new step size by a value of 0.8 or 0.9 has the effect of increasing the probability that $h_{\text {new }}$ will satisfy the error tolerance[47].

$$
\begin{aligned}
& h_{\text {new }}= \begin{cases}h_{\text {old }} \cdot \frac{1}{2}, & \text { if error }>\text { error }_{\max } \\
h_{\text {old }} \cdot 2, & \text { if } \text { error }<\text { error }_{\min } \\
h_{\text {old }}, & \text { otherwise }\end{cases} \\
& h_{\text {opt }}=h \cdot\left(\frac{\text { tol }}{\text { error }}\right)^{\frac{1}{q+1}} \\
& h_{\text {opt }}=h \cdot\left(\frac{\text { tol }}{\text { error }}\right)^{\frac{1}{q+1}} \cdot \text { factor }
\end{aligned}
$$

The code developed in this thesis uses the RK18 method to integrate the equations of motion and heat and mass transfer, with an initial time step of 5 seconds. The maximum relative error tolerance (used to determine whether a time step is small enough) is set to $1.0 \cdot 10^{-7}$; the minimum relative error (used to determine whether increasing the time step is acceptable) is set to $1.0 \cdot 10^{-10}$, both calculated using Equation 3.16.

$$
\text { error }=\max \left(\left|\frac{\hat{y}-y}{y}\right|\right)
$$




\subsubsection{Perturbations}

Unperturbed motion of a two-body system in an inertial frame can be described by Eq. 3.17 , derived from Newton's law of gravity[51], where $\vec{r}$ is the vector from the center of the Earth to the orbiting body, and $\mu$ is the gravitational parameter (the product of the universal gravitational constant and the mass of the central body)- for Earth,

$\mu \approx 398600 \frac{\mathrm{km}^{3}}{\mathrm{~s}^{2}}$. Perturbations can be broken into three main types: those that cause the orbital plane to change, those that cause the period to change, and those that change the eccentricity vector.

Orbital perturbations typically modeled for a spacecraft in Earth orbit can include: atmospheric drag (and lift), solar radiation pressure, Earth oblateness, Earth albedo (reflected radiation), third body gravity (e.g. Moon, Sun, or Jupiter), and Earth magnetic field effects[52].

$$
\begin{aligned}
& \ddot{\vec{r}}=-\frac{\mu}{r^{2}} \cdot \hat{r} \\
& \ddot{\vec{r}}=-\frac{\mu}{r^{2}} \cdot \hat{r}+\vec{p} \\
& \ddot{\vec{r}}=-\frac{\mu}{r^{2}} \cdot \hat{r}-\frac{F_{D}}{m} \cdot \hat{v}
\end{aligned}
$$

It will be assumed that the reentry body does not rotate about any axis. This assumption is made to simplify the analysis; however, this is almost certainly not the case for uncontrolled reentry, which is the limiting case for meeting the requirements specified in Section 1.2.

Since this thesis is concerned with objects that are reentering the atmosphere (125 kilometers altitude and lower), the only perturbing force being modeled is atmospheric drag (Eq. 3.19). The acceleration due to drag is broken into x-, y-, and z-components based on the direction of the velocity vector. The drag force, $F_{D}$, is determined from a quasi-analytical method (detailed in Section 3.3). The lift force is not included because 
only symmetrical bluff bodies at zero angle of attack are modeled at this point. The addition of more general aerodynamics and heat transfer models will allow for the lift force to be captured in the future.

\subsection{Aerothermodynamics}

\subsubsection{Analytical Models}

As a first attempt to solve the reentry modeling problem, some empirically-developed analytical models were used. A Direct Simulation Monte Carlo flow simulation of the scale required for this project takes between one minute and two weeks, depending on altitude; using instead the equations shown below, takes on the order of milliseconds. Thus, using this simplified (but somewhat less accurate) model can result in as little as $10^{-6}$ as much computational time to perform the analysis.

\subsubsection{Atmospheric Drag}

Three flow regimes are identified for this thesis: free molecular flow (here defined as Kn $>10)$, continuum flow $(\mathrm{Kn}<0.001)$, and transitional flow $(0.001<\mathrm{Kn}<10)$.

\subsection{Sphere}

Drag on the sphere in the free molecular regime is modeled as a constant value of 2.12 , taken from a fit line in Figure 2 of [53]. The continuum regime uses a modified Newtonian method to estimate the pressure coefficient distribution on the ram surface (Equation $3.24[19]$ ). The pressure coefficient distribution (normalized to $c_{p, 0}=1$ ) predicted by the modified Newtonian theory is shown in Figure 3.2. Equation 3.23 shows how to calculate the area-weighted average pressure coefficient of the sphere, and shows that the overall effective pressure coefficient of the sphere is one-half that at the stagnation point (defined in Equation 3.25). The drag coefficient is not directly computed in transitional flow, but instead a logarithmic blending function between the free-molecular and continuum values is used (see Equation 3.21). This blending function was developed as a logarithmic-linear 
fit between $0.001<K n<10$ to the data presented in (Figure 3 of [54]).

$$
\begin{aligned}
& C_{D_{F M}}=2.12 \\
& (K n>10) \\
& C_{D_{T}}=C_{D_{C}}+\frac{1}{5}\left(C_{D_{F M}}-C_{D_{C}}\right) \cdot \log (10000 \cdot K n) \quad(0.001<K n<10) \\
& C_{D_{C}} \approx \overline{c_{p}} \\
& (K n<0.001) \\
& \overline{c_{p}}=\frac{\int_{0}^{R} \int_{0}^{\pi} c_{p}(\theta) r d \theta d r}{\int d A}=\frac{c_{p, 0}}{2} \\
& c_{p}(\theta)=c_{p, 0} \sin ^{2} \theta \\
& c_{p, 0}=\frac{p_{0,2}-p_{\infty}}{q_{\infty}} \\
& p_{0,2}=p_{0,1}\left(\frac{\frac{\gamma+1}{2} M_{1}^{2}}{1+\frac{\gamma-1}{2} M_{1}^{2}}\right)^{\frac{\gamma}{\gamma-1}}\left(\frac{1}{\frac{2 \gamma}{\gamma+1} M_{1}^{2}-\frac{\gamma-1}{\gamma+1}}\right)^{\frac{1}{\gamma-1}} \\
& p_{0,1}=p_{\infty}\left(1+\frac{\gamma-1}{2} M_{1}^{2}\right)^{\left(\frac{\gamma}{\gamma-1}\right)} \\
& q_{\infty}=\frac{1}{2} \rho_{\infty} v_{\infty}^{2} \\
& K n=\frac{k_{B} T}{\sqrt{2} \pi d^{2} p L} \approx \frac{\left(2.71877 \cdot 10^{-5}\right) T}{p L}
\end{aligned}
$$

In the above equations, $K n$ is the Knudsen number (computed using Equation 3.29) and $c_{p, 0}$ is the stagnation point pressure coefficient, calculated using Equations 3.25, 3.26, 3.27, and 3.28. A plot of drag coefficient against altitudes from $62 \mathrm{~km}$ to $120 \mathrm{~km}$ has been generated, seen in Figure 3.3. The freestream velocity is $7.5 \frac{\mathrm{km}}{\mathrm{s}}$ at each point; the Knudsen number and density at each altitude are listed in Table 3.4. 


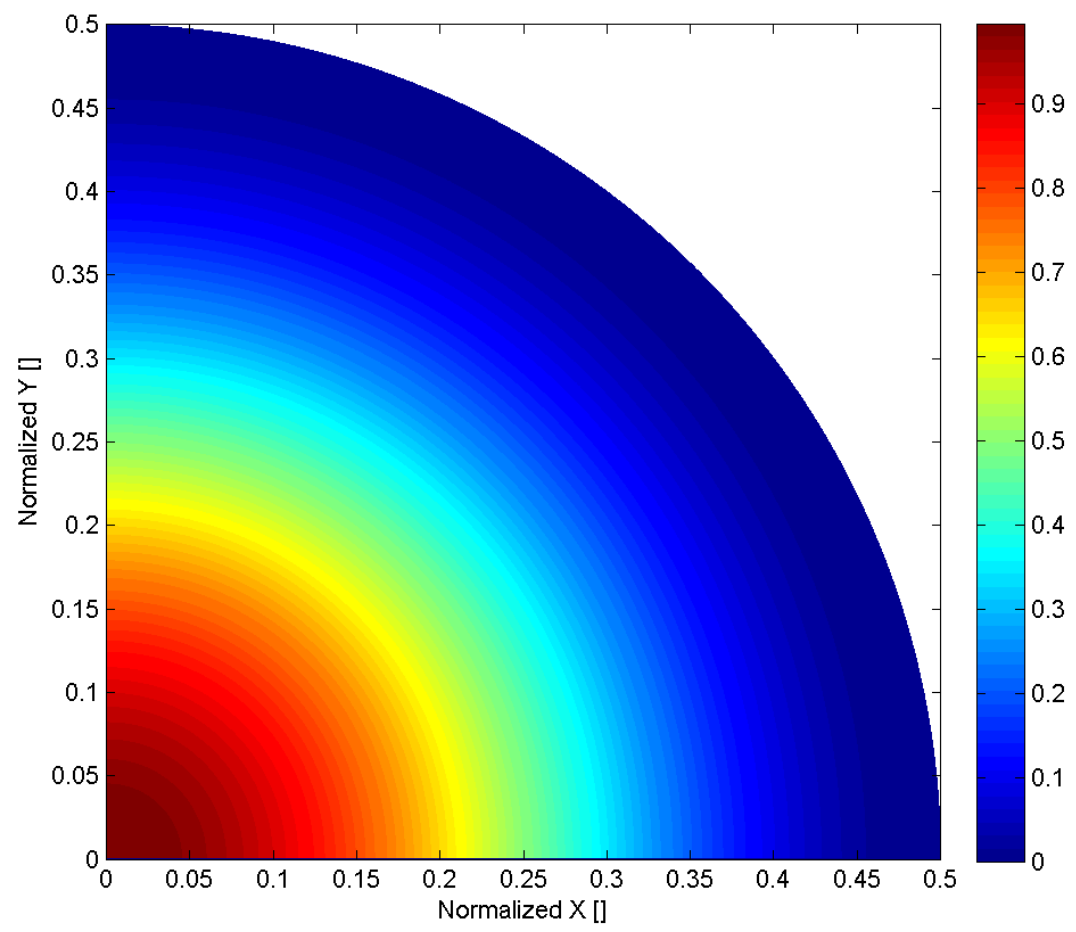

Figure 3.2: Variation of Normalized $c_{p}$ over Ram Surface of Sphere

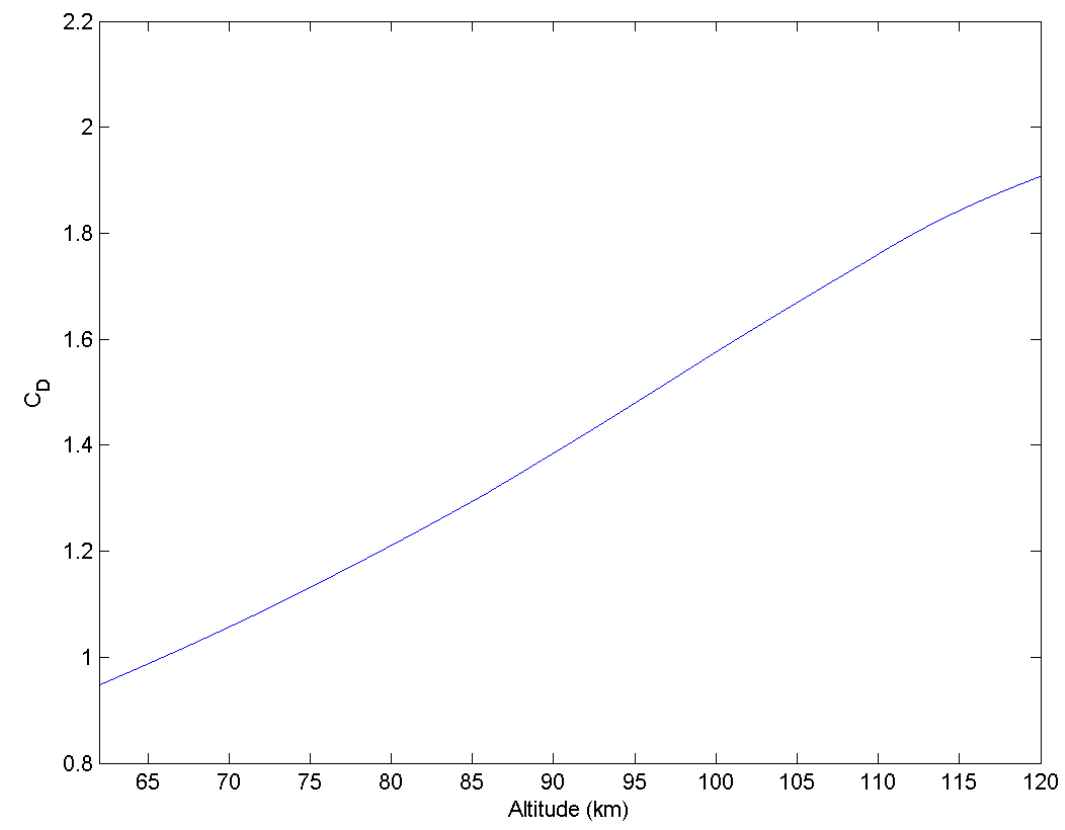

Figure 3.3: Variation of Sphere Drag Coefficient with Altitude 


\section{Table 3.4: $K n$ and $\rho$ for selected altitudes}

\begin{tabular}{|c|c|c|}
\hline Altitude $[\mathrm{km}]$ & $\mathrm{Kn}[]$ & $\rho\left[\frac{\mathrm{kg}}{\mathrm{m}^{3}}\right]$ \\
\hline 62 & 0.001 & $2.407 \mathrm{e}-04$ \\
73 & 0.006 & $5.382 \mathrm{e}-05$ \\
78 & 0.013 & $2.524 \mathrm{e}-05$ \\
88 & 0.065 & $4.866 \mathrm{e}-06$ \\
92 & 0.132 & $2.388 \mathrm{e}-06$ \\
95 & 0.225 & $1.391 \mathrm{e}-06$ \\
100 & 0.553 & $5.594 \mathrm{e}-07$ \\
105 & 1.31 & $2.321 \mathrm{e}-07$ \\
115 & 6.79 & $4.283 \mathrm{e}-08$ \\
120 & 12.87 & $2.220 \mathrm{e}-08$ \\
\hline
\end{tabular}

\subsection{Cube}

The cube model is also separated into three regimes: free molecular, transitional, and continuum, with the same cutoff Knudsen numbers as the sphere model. The main differences between the cube and sphere drag models are the free molecular drag coefficient (here 2.15 instead of 2.12 before), and the constant $c_{p}$ distribution over the ram surface of the cube (since the angle of attack is assumed to be zero). The variation of the cube's drag coefficient with altitude is shown in Figure 3.4a, using the same conditions as listed in Table 3.4.

$$
C_{D_{F M}}=2.15 \quad(K n>10)
$$




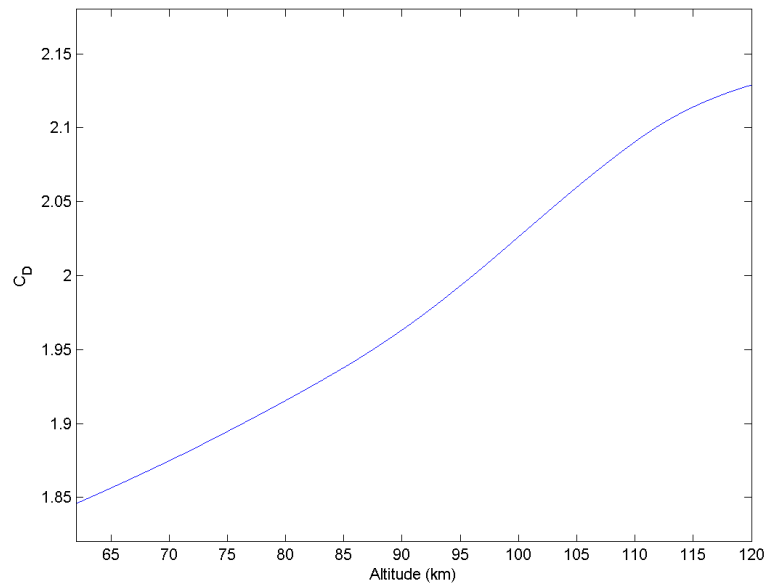

(a) Cube

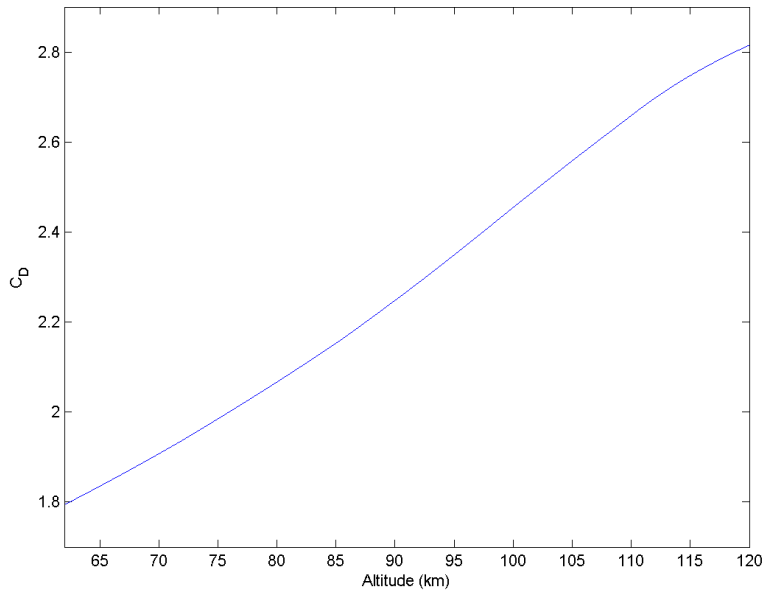

(b) Cylinder

Figure 3.4: Variation of Drag Coefficient with Altitude

\subsection{Cylinder}

The cylinder uses the same drag model as the cube, only differing in the free molecular regime drag coefficient of 3.06 instead of 2.15. The variation of the cylinder's drag coefficient with altitude is shown in Figure 3.4b, using the same conditions as listed in Table 3.4.

$$
C_{D_{F M}}=3.06 \quad(K n>10)
$$

\subsubsection{Heat Transfer}

\subsection{Sphere}

The stagnation point heating rate in hypersonic rarefied flow is approximated by Equation 3.32 (Equation 9.1.2 in [55]), where $c$ is a parameter related to atmospheric composition and varies by planet (Earth has $c=1.83 \cdot 10^{-4} \mathrm{~kg}^{\frac{1}{2}} \cdot \mathrm{m}^{-1}$ and Mars has $c=$ $\left.1.89 \cdot 10^{-4} \mathrm{~kg}^{\frac{1}{2}} \cdot \mathrm{m}^{-1}\right), \rho$ is atmospheric density in $\frac{\mathrm{kg}}{\mathrm{m}^{3}}, \rho_{n}$ is the radius of curvature of the wall in the flow in $m, V$ is the flow speed in $\frac{m}{s}$, and $q^{\prime \prime}$ is the heat flux, measured in $\frac{W}{m^{2}}$. The heat flux varies over the body, as a function of the local angle between the wall and the flow (here denoted as $\theta$ ), as in Equation 3.33.[56] Discretizing the surface 
of the sphere then integrating Equation 3.33 results in Equation 3.34. The approximate form of Eq. 3.34 was found by using a surface mesh with 25 million faces, summing the contribution from each face, and then normalizing to a unit area. Calculating this value prior to any computation avoided the need to create a surface mesh and iterate over all faces at each time step.

$$
\begin{aligned}
q_{s}^{\prime \prime} & =c \sqrt{\frac{\rho}{\rho_{n}}} V^{3} \\
q^{\prime \prime}(\theta) & =q_{s}^{\prime \prime}(0.1+0.9 \cos \theta) \\
\dot{Q} & \approx \sum_{i=0}^{N} q^{\prime \prime}\left(\theta_{i}\right) \delta A_{i} \approx 0.6745 q_{s}^{\prime \prime} A \\
C_{h} & =\frac{\dot{Q}}{\frac{1}{2} \rho v^{3}}
\end{aligned}
$$

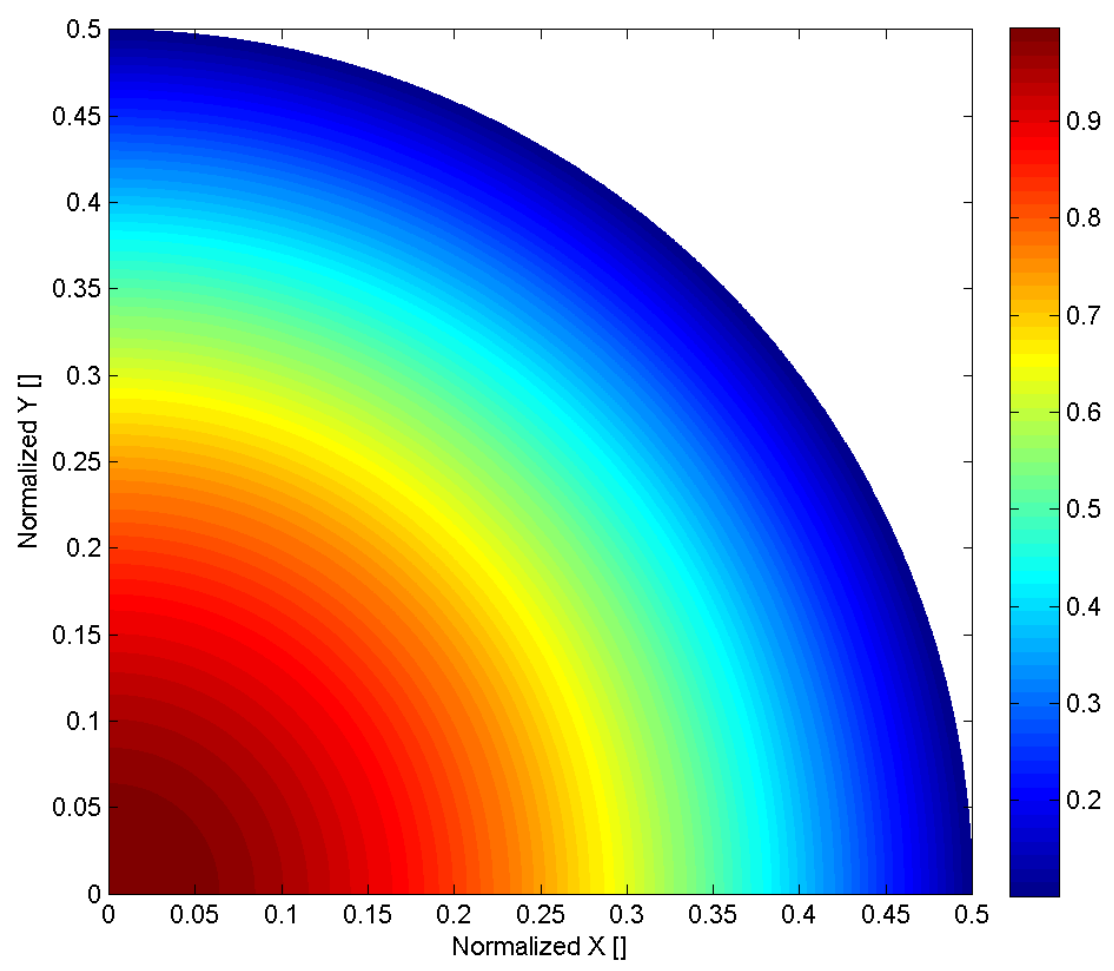

Figure 3.5: Variation of Normalized Heat Flux over Ram Surface of Sphere 


\subsection{Cube}

Equation 3.32 can be used to approximate the stagnation point heat transfer on a blunt body, but is inaccurate for bodies with flat ram surfaces, like cubes or cylinders. Mehta et al[56] developed a new model for the distribution of heat transfer coefficient $C_{h}$ (defined in Equation 3.35) over the ram surface of a cube, described in Equations 3.37, 3.38, and 3.39, and plotted with filled contours in Figure 3.6b. Since this distribution is not expected to change as a function of altitude, speed, or density, the average was computed to reduce the number of calculations during the simulation, using the same method as with the drag coefficient of the sphere - discretizing the surface into many small faces, adding all their contributions, then dividing by total area (see Eq. 3.40). The approximate value seen in Eq. 3.40 is normalized to the stagnation heat flux, and must be rescaled at each timestep to obtain the correct heat flux over the ram surface.[56] The cube ram surface stagnation point heat flux coefficient varies with altitude and speed as in Figure 3.6a (interpolated from Figure 7b in [56]).

$$
\begin{aligned}
q^{\prime \prime} & =\frac{1}{2} \rho_{\infty} C_{h} v_{\infty}^{3} \\
C_{h}(x, y) & =\sqrt{C_{h}^{2}(x)+C_{h}^{2}(y)} \\
C_{h}(x) & =0.0079\left(e^{5.627|x|}-e^{-2.000|x|}\right) \\
C_{h}(y) & =0.0079\left(e^{5.627|y|}-e^{-2.000|y|}\right) \\
C_{h, t o t} & \approx \frac{\sum_{i=1}^{N} C_{h}\left(x_{i}, y_{i}\right) \delta A_{i}}{\sum_{i=1}^{N} \delta A_{i}} \approx 1.031 \cdot C_{h, s}+0.0665
\end{aligned}
$$




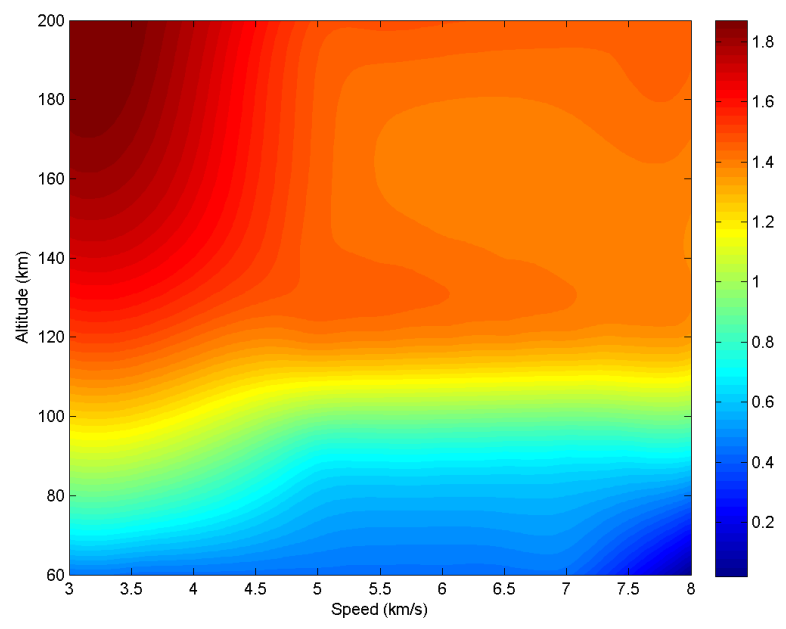

(a) Altitude - Speed Variation

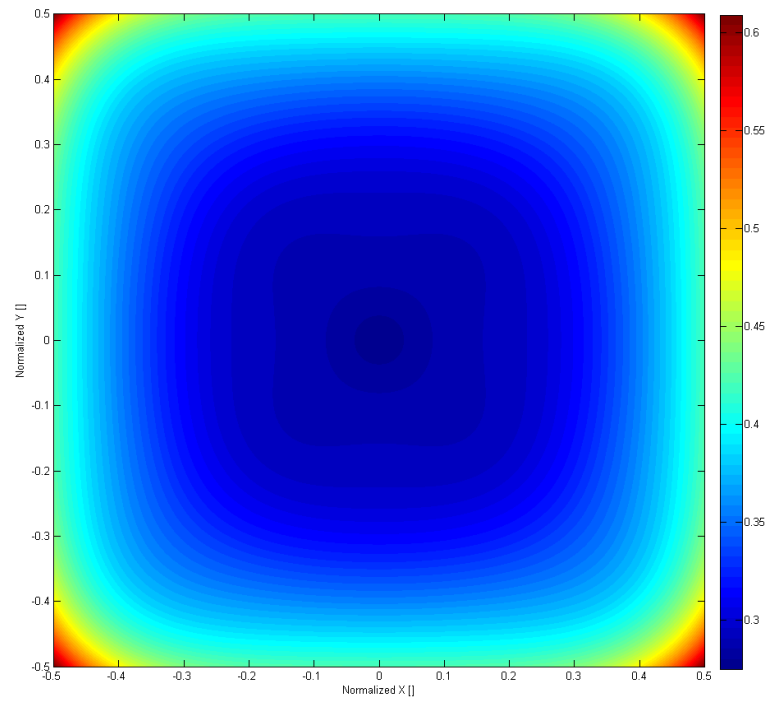

(b) Ram Surface Variation

Figure 3.6: Variation of $C_{h, s}$ (Cube)

\subsection{Cylinder}

The blunt-body heat transfer approximation found in Equation 3.32 is inaccurate for cylinders, but a new model, very similar to the cube heat transfer model above, was developed that described the distribution of the heat transfer coefficient $C_{h}$ over the ram surface of a cylinder.[56] The local heat transfer coefficient is solely a function of radius, since the cylinder is radially symmetric (see Equation 3.41). As with the cube, an expression for the average heat transfer coefficient in terms of the stagnation point coefficient was created, using Equation 3.42, to reduce the calculations during the simulation. A continuous model for $C_{h, s}$ as a function of altitude and speed was then created using data from Figure 8b from [56] and MATLAB's built in gridded interpolation scheme interp2 with the bicubic method flag, the results of which can be seen in Figure 3.7a.

$$
\begin{aligned}
C_{h}(r) & =\left(4.744 \cdot 10^{-8}\right) e^{30.397 r}-0.004744 e^{-9.161 r}-6.850 \sin (-0.02210 r)+0.298 \\
C_{h, t o t} & \approx \frac{\sum_{i=1}^{N} C_{h}\left(r_{i}, \theta_{i}\right) \delta A_{i}}{\sum_{i=1}^{N} \delta A_{i}} \approx 0.04935 C_{h, s}
\end{aligned}
$$




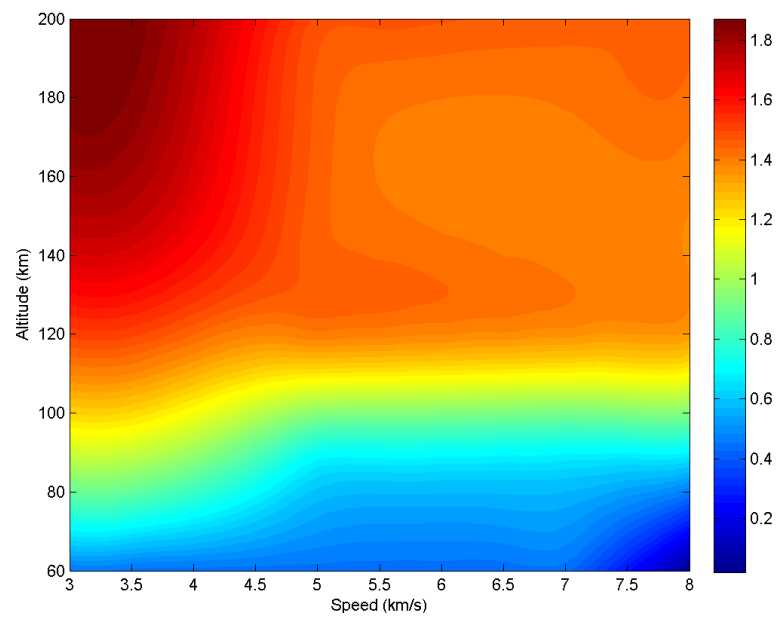

(a) Altitude - Speed Variation

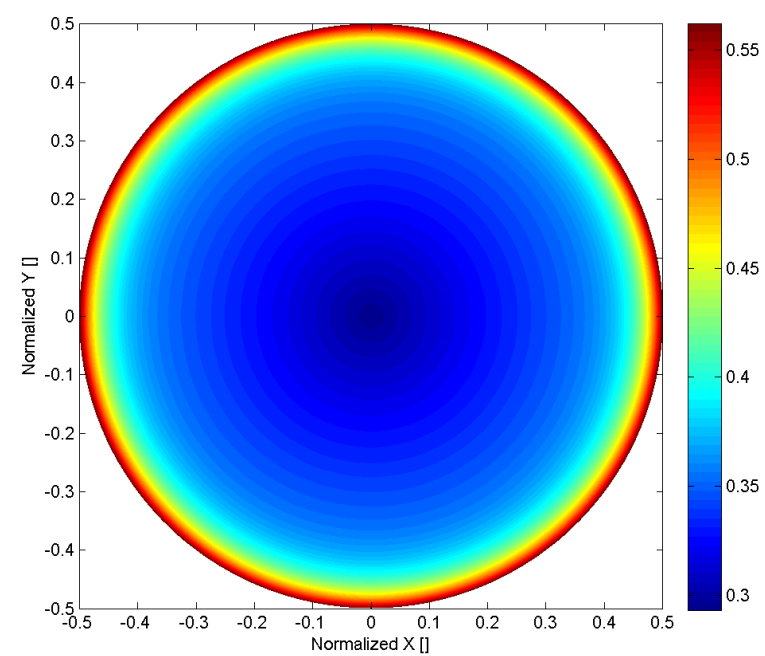

(b) Ram Surface Variation

Figure 3.7: Variation of $C_{h, s}$ (Cylinder)

\subsubsection{Direct Simulation Monte Carlo Method}

The DSMC method was first proposed in 1960 by Dr. Graeme Bird as a way to probabilistically simulate gas flows[57]. It is similar to the Molecular Dynamics (MD) method, in that it tracks particles, but DSMC does not track trajectories and collisions deterministically[58]. Since this method does not directly solve the governing equations of fluid flow but instead simulate them stochastically, its validity was questioned, and it was not proven mathematically until 1990 that the DSMC method converges in the limit to a solution of the Boltzmann equation[59].

The accuracy of a DSMC simulation relies on the number of particles used to estimate the properties of the flow field $[60,61]$; however, the number of particles in a DSMC simulation (and hence the number of cells) depends on the amount of memory a machine has access to; the time required to converge a solution, like in other, more conventional schemes, increases rapidly with the number of cells in the mesh[58]. It is easy, then, to see that an accurate solution using DSMC may be prohibitively computationally expensive.

As general-purpose computers became more and more powerful in the 1990's and 
2000's, DSMC became more popular. More complex geometries, flow conditions[62, 63], and even chemical reactions $[64,65,66]$ can now be solved using DSMC. The advent of many-core architectures (both CPU and GPU) further spurred DSMC research toward reducing runtime while maintaining fidelity. [67, 68, 69, 70, 71, 72, 73]

\subsubsection{Algorithm}

The DSMC method is a technique for approximating the solution to the Boltzmann equation, which describes non-equilibrium thermodynamic systems.[74] The original algorithm proposed by Bird is as follows (reproduced from [57]):

\section{The DSMC Method}

1. Advance flow by minimum time step

2. Introduce new molecules at any flow entry boundaries

3. Loop over the molecules:

(a) If the molecule time is more than one local time step behind the flow time, move the molecule through the distance appropriate to twice the local time step

(b) Act on any boundary and surface interactions

(c) Sample surface properties

4. Index molecules to the collision and sampling cells

5. Loop over the collision cells:

(a) If the cell time is more than one local time step behind the flow time, calculate collisions and reactions appropriate to twice the local time step

6. If flow time is greater than the next sample time, go to 1 
7. Sample the flowfield properties

8. If flow time is greater than the next output time, go to 1

9. Output the flow, surface, and flowfield results

10. Update the time steps

11. Reset the samples (if unsteady flow application)

12. If the sample size is sufficient in a steady flow application, or if the desired time limit has been reached, stop the simulation. Otherwise, go to 1 and continue

\subsubsection{Applications to Atmospheric Entry Flows}

Entry flows are generally characterized by high Mach numbers, low freestream density, and high Knudsen numbers. Accurately predicting drag forces and surface heat fluxes is critical for surviving the entry environment (or ensuring demise of an object during entry), and in this non-continuum regime, the DSMC method stands out.

Analytical models can be used (like Newton's method) in the prediction of hypersonic flows over certain shapes and flow conditions with reasonable agreement[75]; numerical methods can be used over general bodies in general flow conditions with varying success. Reentry experiments have been conducted that use common base shapes (like cones, spheres, and sphere-blunted cones); the results of these experiments (surface pressures and heating rates) were compared against DSMC simulations, Navier-Stokes, and Viscous Shock Layer solutions, with DSMC most closely following the experimental data at altitudes above $80 \mathrm{~km} .[76,77]$

As space travel becomes more profitable and popular, atmospheric entry will continue to be an area of research with lots of attention.[78] 


\subsubsection{Coupling DSMC and Navier-Stokes}

Since the DSMC method can become prohibitively computationally expensive as the characteristic Knudsen number of the flow decreases past 0.01 (either by having low density as in upper atmospheric conditions or very small characteristic lengths like in microchannels), there have been attempts to couple DSMC solvers with continuum Navier-Stokes solvers[79, 80]. These hybrid schemes gain the accuracy of a DSMC simulation in the rarefied regions of the flowfield while keeping the lower computational expense of a continuum algorithm. Similarly to the research that has been done in each flow regime (continuum and rarefied), parallelization is a growing area of research for these hybrid solvers[81].

\subsubsection{OpenFOAM}

OpenFOAM is a toolbox (written in $\mathrm{C}++$ ) for numerical solvers, including differential equations, finance, and continuum mechanics (like CFD). It is a product of OpenCFD Ltd. and the OpenFOAM Foundation, which develop, license, and distribute the software freely via the Internet. OpenFOAM has been used widely in both academia and industry since it does not have cost as a barrier to access like other commercially developed solvers. Like other publicly-available CFD packages, OpenFOAM has been designed for parallel processing, on CPU and recently on GPU.[82]

The solver initially proposed for use in this thesis, dsmcFoam, was released in 2010 as part of OpenFOAM, with the intent of being fully-capable and customizable.[64, 83] Since its release, it has been tested for both speed and accuracy, with satisfying results. [84, 85, 86] Following the work of prior multi-region and coupled OpenFOAM solvers, development of hybrid particle-continuum schemes started almost immediately after the release

of dsmcFoam. [87, 88, 89] Despite these improvements in parallelism and hybridization, the computational cost of using a full DSMC solver is too great to be used during a simulation, and is not included in this thesis' code. 


\subsection{Ablation}

Ablation is the removal of material from a surface, through vaporization, chipping due to stress, or by other erosion. The ablative properties of materials have been used to ensure that human and mechanical payloads return safely from spaceflight (and conversely, that certain objects do not survive their atmospheric reentry).

\subsubsection{Materials Database}

Once surface temperatures are found from the aerothermodynamic analysis, the material's thermal conductivity is needed to determine the rate and amount of heat transfer throughout the body. To this end, a database of the most common aerospace materials (the 77 materials included in NASA's DAS User Guide[23] and reproduced in Appendix D) has been compiled and added as a module to this thesis. The list of materials includes four pieces of information: specific heat capacity, melting point temperature, specific heat of fusion, and density.

\subsubsection{Ablation Modeling}

Surface heat fluxes are determined using the analytical models discussed in Section 3.3.1.2 and added to the prior time step (see Equation 3.43). Once the melting point internal energy $e_{\text {ablation }}$ has been reached for an element, it is assumed to ablate away, and is removed from the model of the reentry object. The mass of the object is calculated at each time step by calculating the remaining volume of the object and multiplying by the density of the material(Equation 3.46). 


$$
\begin{aligned}
\dot{Q}(x, y, z) & =\int q^{\prime \prime}(x, y, z) d A-\dot{Q}_{r a d} \\
\dot{Q}_{r a d} & =\sigma \epsilon_{0} A\left(T^{4}-T_{s}^{4}\right) \\
T_{s} & =T_{\infty}\left(1+\frac{\gamma-1}{2} M_{\infty}^{2}\right) \\
m\left(t_{i}\right) & =m\left(t_{i-1}\right)-\rho \cdot \delta V\left(t_{i}\right)
\end{aligned}
$$

This model for ablation is not physical in nature, since the outgassing of the surface is not accounted for, nor is the erosion of the object from aerodynamic stresses, nor is the

effect of the boundary layer (consisting of air and gaseous, liquid, and solid material). The ablated material is also assumed to not redeposit back onto the body. Recent research in 2-D hypersonic ablation suggests higher accuracy can be achieved by modeling three regions: the boundary layer, the melt layer, and the ablating solid.[90]

\subsection{OSTROM}

After discussing in detail each component of the code presented in this thesis, an overview of the software package can be presented. This software is designed to only require the user to input the initial radius and velocity vectors, the shape of the object, the material of the body, and several uncertainty ranges they wish to constrain the simulation with (see Appendix B for a sample case file). 


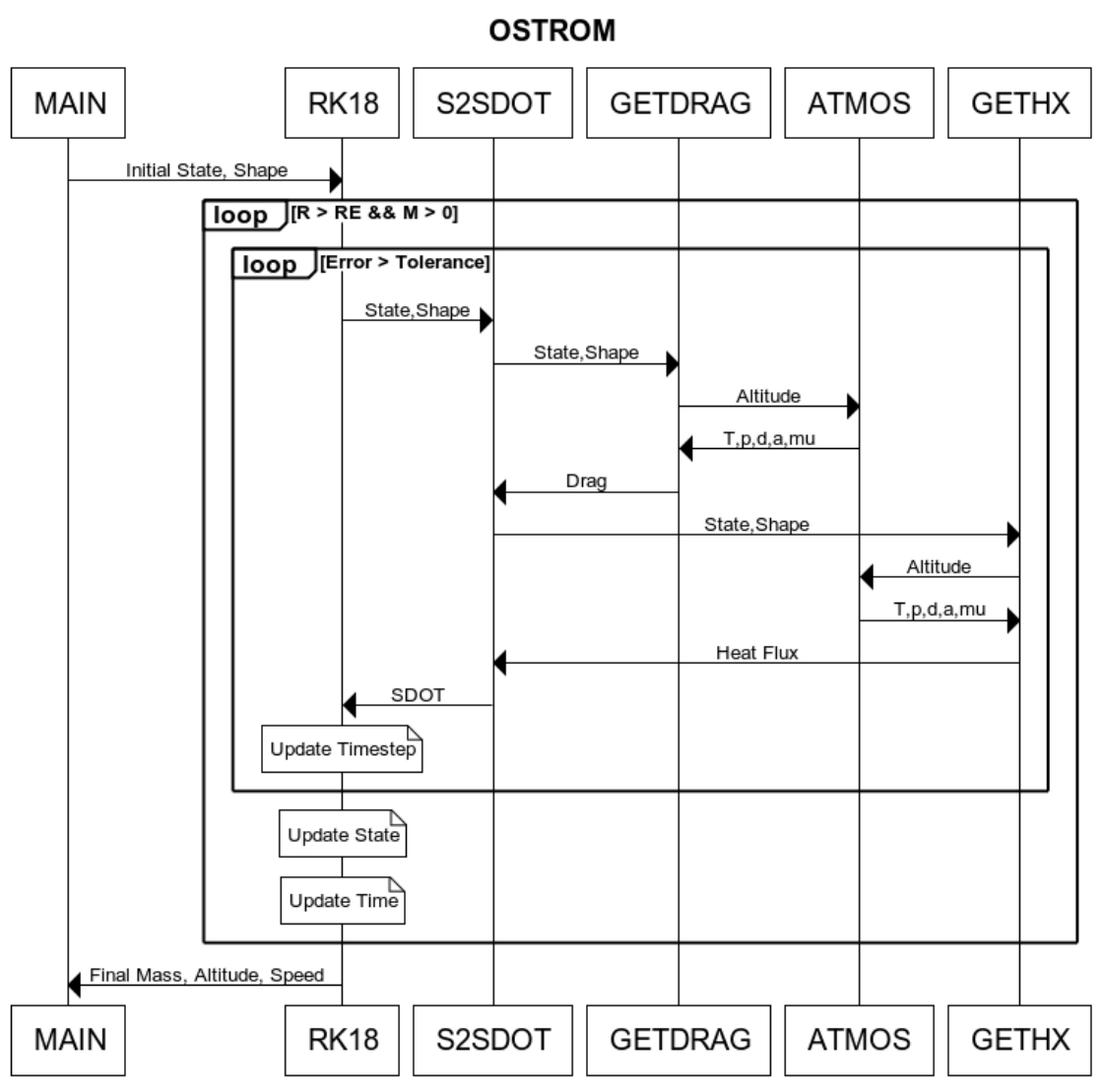

Figure 3.8: Software Architecture of OSTROM

OSTROM is written in the $\mathrm{C}++$ language using the $\mathrm{C}++11$ standard with the architecture seen in Figure 3.8. The algorithm used to simulate the reentry of the userprovided object is seen below. The state vector used for the simulation is as seen in Eq. 3.47 below, where $\vec{r}$ is the radius vector, $\vec{v}$ is the velocity vector, $u$ is the internal energy of the body, and $m$ is the mass of the body.

Prior iterations of the code developed for this thesis included a Monte Carlo campaign of trajectories, using as many as 1000 runs per combination of material and shape to determine the reentry envelope. Changing to a higher order integration scheme (and decreasing the error tolerance) caused the runtime to increase dramatically (to between 5-10 minutes per trajectory, or 5000-25000 timesteps per trajectory). After some testing, it was found that several trajectories could be run in parallel (up to the hardware con- 
currency) without much extra work, but running a full campaign would take more than a half day for a single test case on a consumer-grade computer, and so was excluded for the current version.

$$
\vec{S}=\left[\begin{array}{l}
\vec{r} \\
\vec{v} \\
u \\
m
\end{array}\right]
$$

\subsubsection{Algorithm}

1. Read the case.txt file prepared by the user for initial radius, velocity, and uncertainty estimates for those quantities

2. Check the thread concurrency on the user's machine (typically 4 or 8 for modern consumer computers)

3. Create a thread for each subset of the total runs to be performed (each subset contains the total number of runs, divided by the number of concurrent threads to be run)

In each thread:

(a) Inject random error into the radius and velocity vectors' components based on the user-specified uncertainties (Equations 3.48, 3.49)

(b) Copy the case folder containing the user's 3-D model to a new folder

(c) Run a trajectory by calling $r k 78$ with the new $\tilde{\vec{r}}, \tilde{\vec{v}}$ vectors

(d) Return the state history of each trajectory to the parent function

4. Find the average of the final states for the trajectories

5. Write results file to results.txt 


$$
\begin{aligned}
& \tilde{\vec{r}}=\overrightarrow{r_{0}}+\vec{\epsilon}\left(\overrightarrow{r_{0}}\right) \\
& \tilde{\vec{v}}=\overrightarrow{v_{0}}+\vec{\epsilon}\left(\overrightarrow{v_{0}}\right)
\end{aligned}
$$




\section{Chapter 4}

\section{Results}

The following data and graphs were collected after conducting simulations for each of the 77 materials in the database and 3 solid shapes (sphere, cube, and cylinder). Papers comparing ORSAT and SCARAB include graphs that demonstrate how the test case results, like impact mass and demise altitude, differ, but are not labeled with test case numbers. The code presented in this thesis is not currently capable of performing a full-satellite analysis for comparison with other destructive reentry software, like seen in [26], but simple test cases, like those below, are compared with results from DAS.

After reviewing the graphs produced by the code, Acrylic, Silver, and Molybdenum were chosen as the three representative materials to be shown: acrylic demises at a high altitude, silver, which demises at a low altitude, and molybdenum, which survives to impact the surface with its entire initial mass. These three materials were not only chosen qualitatively, but also because each possessed the combination of characteristics most likely to decide demise or survival: specific heat capacity, latent heat of fusion, and melting point temperature, which are compared in Table 4.1.

Each test object is assumed to have the initial classical orbital elements listed in Table 4.2. The corresponding initial radius and velocity vectors, along with the initial altitude and flight path angle, are listed in Table 4.3. The test objects are placed on the orbit described below at the apogee, at an initial altitude of $78 \mathrm{~km}$, the DAS starting point. 
Table 4.1: Material Properties of Selected Representative Materials

\begin{tabular}{|c|c|c|c|}
\hline Material & Acrylic & Molybdenum & Silver \\
\hline Specific Heat Capacity $\left(\frac{\mathrm{J}}{\mathrm{kg} \cdot \mathrm{K}}\right)$ & 1465 & $231.7-412.0$ & $222.6-243.7$ \\
Latent Heat of Fusion $\left(\frac{\mathrm{J}}{\mathrm{kg}}\right)$ & 0.0 & 293057.0 & 105833.0 \\
Melting Temperature $(\mathrm{K})$ & 505.0 & 2899.0 & 1234.0 \\
Density $\left(\frac{\mathrm{kg}}{\mathrm{m}^{3}}\right)$ & 1170.00 & 10219.0 & 10492.0 \\
Total Ablation Energy $\left(\frac{\mathrm{kJ}}{\mathrm{kg}}\right)$ & 739.8 & $964.8-1,487$ & $380.5-406.6$ \\
\hline
\end{tabular}

Table 4.2: Initial Classical Orbital Elements

\begin{tabular}{|c|c|c|}
\hline Quantity & Value & Unit \\
\hline Semimajor axis $(a)$ & 6449.6 & $\mathrm{~km}$ \\
Eccentricity $(e)$ & 0.0010 & - \\
Specific relative angular momentum $(h)$ & 50703.0 & $\frac{\mathrm{km}^{2}}{\mathrm{~s}}$ \\
Inclination $(i)$ & 60 & $\circ$ \\
Right Ascension of the Ascending Node $(\Omega)$ & 90 & $\circ$ \\
Argument of Periapsis $(\omega)$ & 180 & $\circ$ \\
True Anomaly $(\theta)$ & 180 & $\circ$ \\
\hline
\end{tabular}


Table 4.3: Initial State Vector, Altitude, and Flight Path Angle

\begin{tabular}{|c|c|c|}
\hline Quantity & Value & Unit \\
\hline$r_{x}$ & 3228.0 & $\mathrm{~km}$ \\
$r_{y}$ & 0.0 & $\mathrm{~km}$ \\
$r_{z}$ & -5591.1 & $\mathrm{~km}$ \\
$v_{x}$ & 0.0 & $\frac{\mathrm{m}}{\mathrm{s}}$ \\
$v_{y}$ & 7853.6 & $\frac{\mathrm{m}}{\mathrm{s}}$ \\
$v_{z}$ & 0.0 & $\frac{\mathrm{m}}{\mathrm{s}}$ \\
Altitude & 78.0 & $\mathrm{~km}$ \\
Flight Path Angle $(\gamma)$ & 0 & $\circ$ \\
\hline
\end{tabular}

\subsection{Solid Spheres}

The first test case chosen is a solid sphere of diameter $15 \mathrm{~cm}$. The mass and demise total internal energy (calculated using Equation 1.1) for each test sphere is listed in Table 4.4. The total energy, calculated using Equation 4.1 (Equation 2.81 in [51]), where $v$ is the spacecraft speed in $\mathrm{km} / \mathrm{s}, r$ is the radius in $\mathrm{km}, \mu$ is the standard gravitational parameter in $\frac{k m^{3}}{s^{2}}$, and $e$ is the total specific energy in $\frac{M J}{k g}$, of the body at its initial conditions is -33.6 $\frac{\mathrm{MJ}}{\mathrm{kg}}$ (hence -556 MJ for acrylic, -4986 MJ for silver, and -4856 MJ for molybdenum). Again using Equation 4.1 to calculate the energy at the surface (with zero velocity), the maximum energy available to be converted from potential and kinetic into heat is 478 MJ for the acrylic, $4284 \mathrm{MJ}$ for the silver, and $4172 \mathrm{MJ}$ for the molybdenum. These values are much larger than the values listed for $h_{\text {ablation }}$ in Table 4.4 , but molybdenum is not expected to demise because the vast majority of the energy goes into heating the flow, not the body - Equation 4.2 indicates that only $0.1 \%$ of all the heat energy goes into the body (Equation 1.10 in [91]). 


$$
\begin{aligned}
e_{t o t} & =\frac{v^{2}}{2}-\frac{\mu}{r} \\
\dot{Q}_{b o d y} & \approx 0.001 \dot{Q}_{a t m o s}
\end{aligned}
$$

\section{Table 4.4: Test Sphere Mass and Demise Internal Energy}

\begin{tabular}{|c|c|c|}
\hline Material & Mass $(\mathrm{kg})$ & $h_{\text {ablation }}(\mathrm{MJ})$ \\
\hline Acrylic & 16.54 & 12.24 \\
Molybdenum & 144.5 & $139.4-214.9$ \\
Silver & 148.3 & $56.44-60.30$ \\
\hline
\end{tabular}

\subsubsection{Acrylic}

The demise altitude predicted by OSTROM is $74.4 \mathrm{~km}$, and the demise altitude predicted by the reentry survivability analysis utility in DAS is $73.9 \mathrm{~km}$, as seen in Appendix E. The near-vertical lines seen in Figure $4.1 \mathrm{~b}$ are an artifact of Equation 2.3 and the lumped thermal mass model: no mass is lost by the object until the entire body reaches the melt temperature, at which point all further heat transfer causes ablation. This effect is compounded by the heat of fusion being identically zero for Acrylic (but modeled as 1.0 $\frac{J}{k g}$ in OSTROM as it encountered divide-by-zero errors otherwise). 


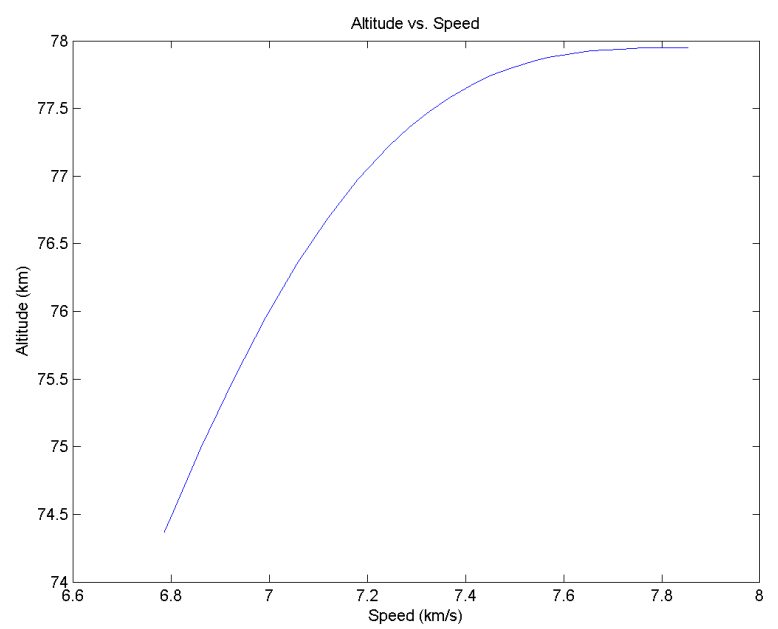

(a) Trajectory during reentry

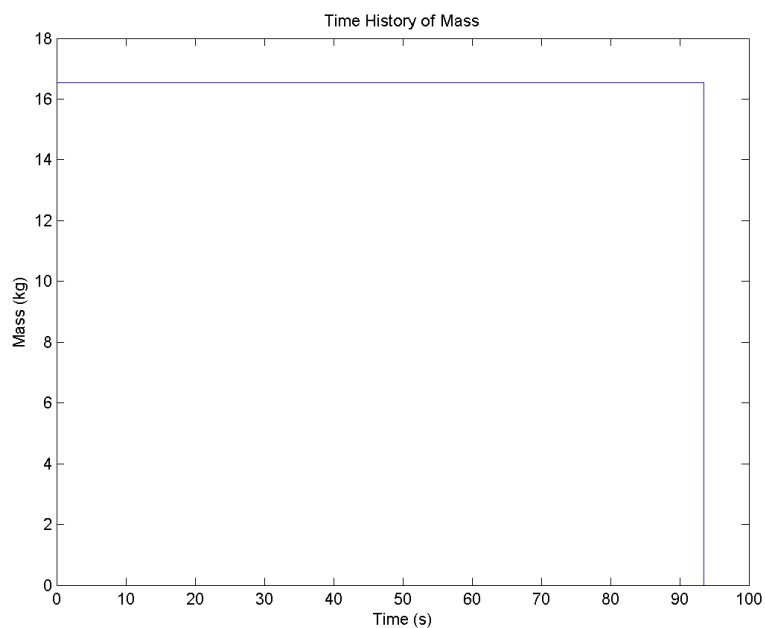

(b) Mass variation during reentry

\section{Figure 4.1: Acrylic Sphere}

\subsubsection{Molybdenum}

As expected from the calculations above, the simulated trajectory does not result in the demise of a molybdenum sphere. The impact speed is approximately $249 \frac{\mathrm{m}}{\mathrm{s}}$, as seen in Figure 4.2a. The kinetic energy at impact is predicted to be $4.48 \mathrm{MJ}$; DAS predicts the kinetic energy of the object at impact to be $4.00 \mathrm{MJ}$, a difference of $12 \%$. This difference is likely due to the different drag models used at altitudes lower than $20 \mathrm{~km}$. Since the molybdenum sphere does not experience a large enough total heat load during the reentry simulation, it does not lose any mass, as indicated by the horizontal lines in Figure 4.2b. 


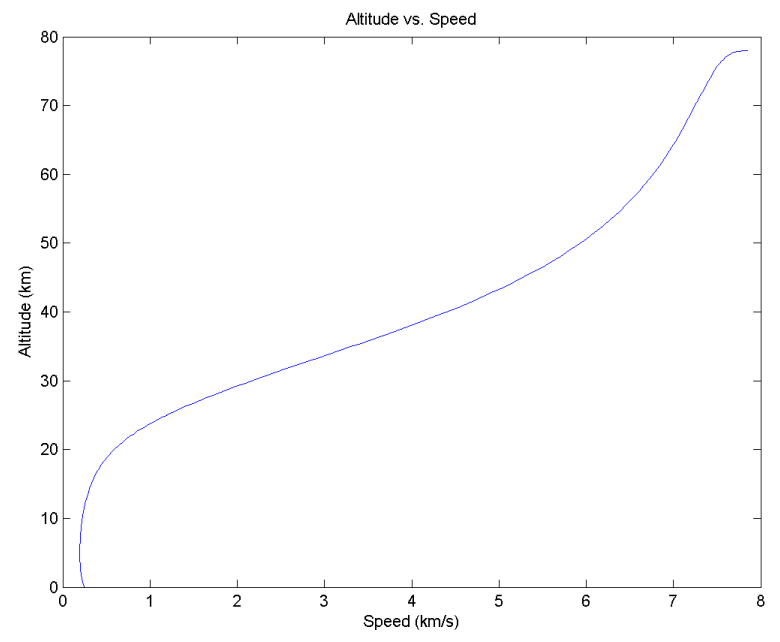

(a) Trajectory during reentry

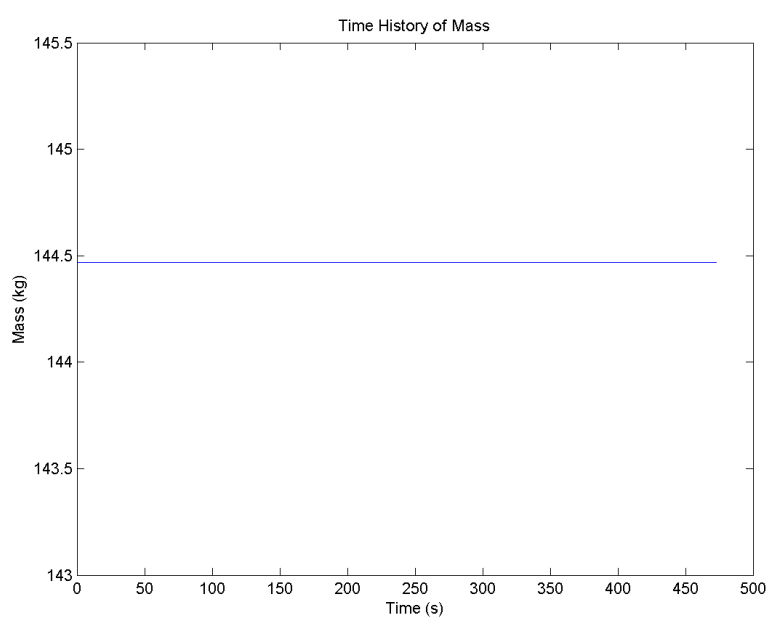

(b) Mass variation during reentry

Figure 4.2: Molybdenum Sphere

\subsubsection{Silver}

The simulated trajectory resulted in the demise of the silver sphere, at an altitude of $42.1 \mathrm{~km}$. For this test case, DAS predicts a demise altitude of $43.2 \mathrm{~km}$. Unlike the acrylic sphere, with its extremely low heat of fusion, the silver sphere has a significant resistance to ablation. Even when the sphere reaches its melting temperature, it does not completely demise instantly, but over a period of minutes, as seen in Figure 4.3b. This behavior is much more realistic than seen in the case of the acrylic sphere, even using the same thermal model. 


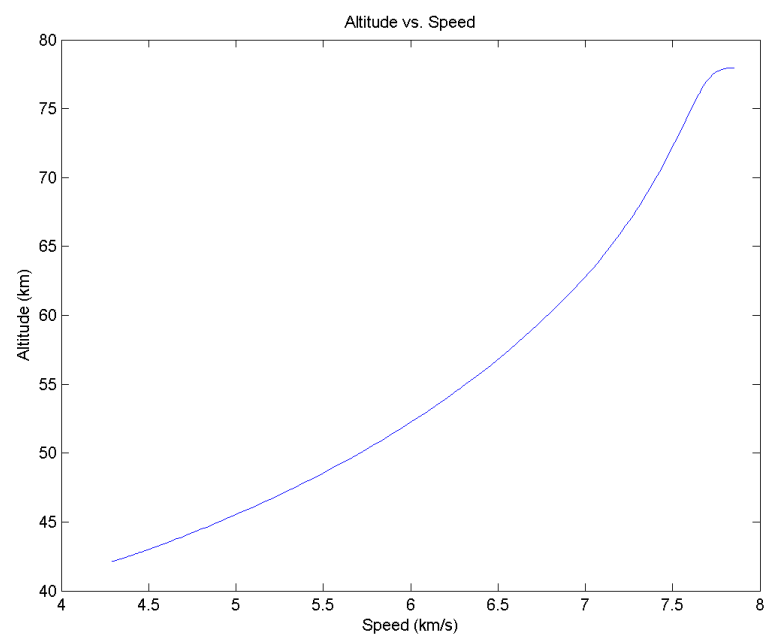

(a) Trajectories during reentry

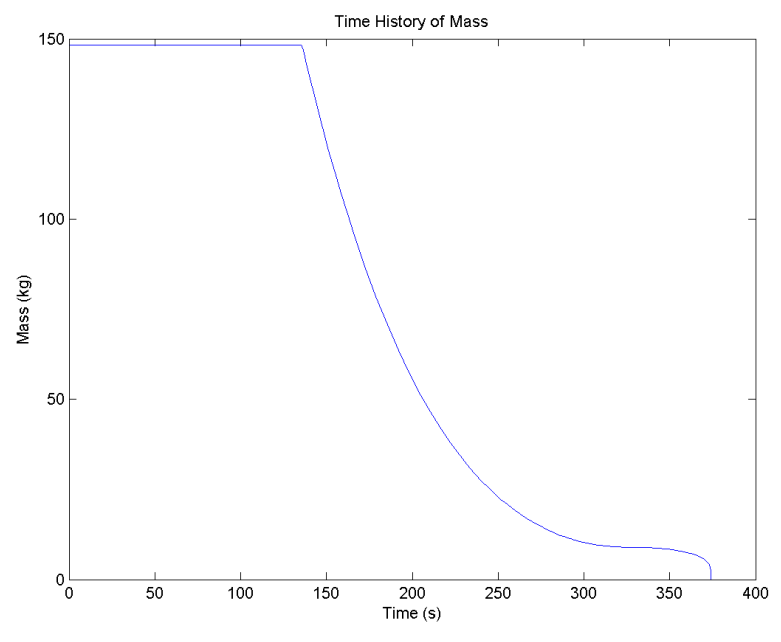

(b) Mass variation during reentry

Figure 4.3: Silver Sphere

\subsection{Solid Cubes}

The second test case chosen is a solid cube of side length $30 \mathrm{~cm}$. The mass and demise total internal energy (calculated using Equation 1.1) for each test sphere is listed in Table 4.5. The total energy, calculated using Equation 4.1 (Equation 1.10 in [91]), of the body at its initial conditions (listed in Table 4.3) is -33.6 $\frac{\mathrm{MJ}}{\mathrm{kg}}$ (hence -1062 MJ for acrylic, -9523 MJ for silver, and -9275 MJ for molybdenum). Again using Equation 4.1 to calculate the energy at the surface (with zero velocity), the maximum energy available to be changed from potential and kinetic into heat is 912 MJ for the acrylic, 8181 MJ for the silver, and $7968 \mathrm{MJ}$ for the molybdenum. With the same reasoning as with the solid spheres, though the amount of energy that will be converted to heat is much greater than $h_{\text {ablation }}$, most of the energy will heat the atmosphere, not the body. 


\section{Table 4.5: Test Cube Mass and Demise Internal Energy}

\begin{tabular}{|c|c|c|}
\hline Material & Mass $(\mathrm{kg})$ & $h_{\text {ablation }}(\mathrm{MJ})$ \\
\hline Acrylic & 31.59 & 23.37 \\
Molybdenum & 275.9 & $266.2-410.4$ \\
Silver & 283.3 & $107.8-115.2$ \\
\hline
\end{tabular}

\subsubsection{Acrylic}

The simulated trajectory resulted in the demise of the acrylic cube, at an altitude of 75.1 $\mathrm{km}$, as seen in Figure 4.4a. DAS predicts total demise of the acrylic cube as well, at an altitude of $74.4 \mathrm{~km}$. As discussed above, the heat of fusion of acrylic is extremely small, and as soon as the cube reaches its melting temperature, the modeled ablation process takes a fraction of a second, evidenced by the vertical lines in Figure 4.4b.

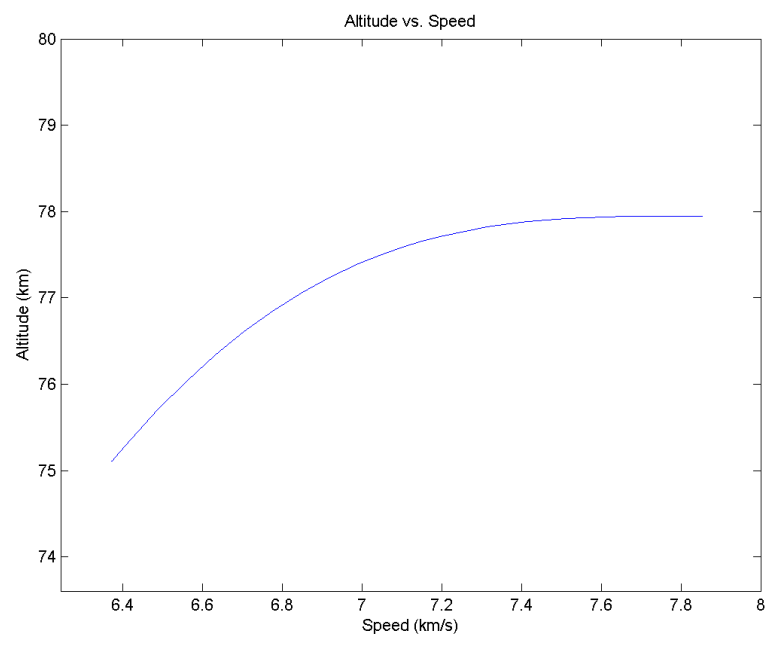

(a) Trajectory during reentry

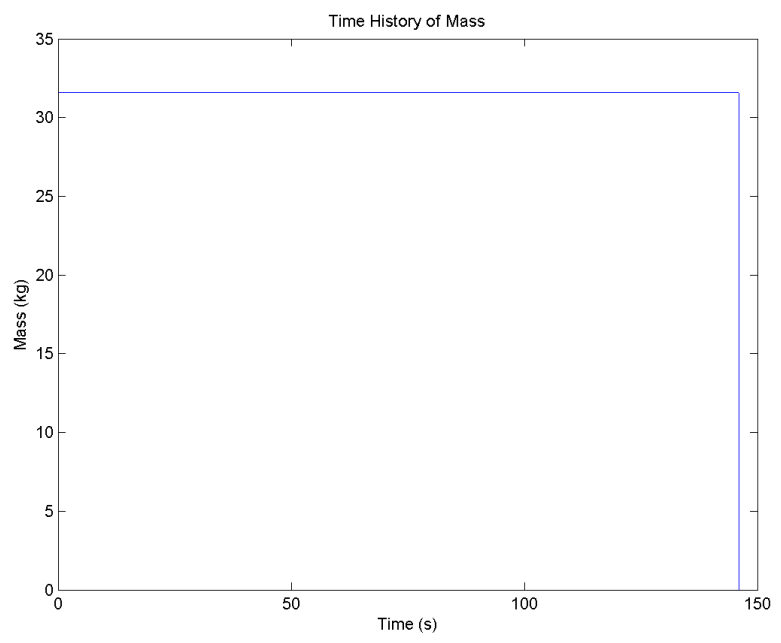

(b) Mass variation during reentry

Figure 4.4: Acrylic Cube

\subsubsection{Molybdenum}

The molybdenum cube did not demise in the simulated trajectory, as seen in Figure 4.5a. The predicted speed on surface impact is $248 \frac{\mathrm{m}}{\mathrm{s}}$ (or an equivalent kinetic energy of 8.485 
MJ), while DAS predicts 6.319 MJ, a difference of 34\%. This error derives from the different drag models used by DAS and OSTROM at the lower altitudes. No mass was ablated during the simulated reentry, demonstrated by a horizontal line in Figure 4.5b.

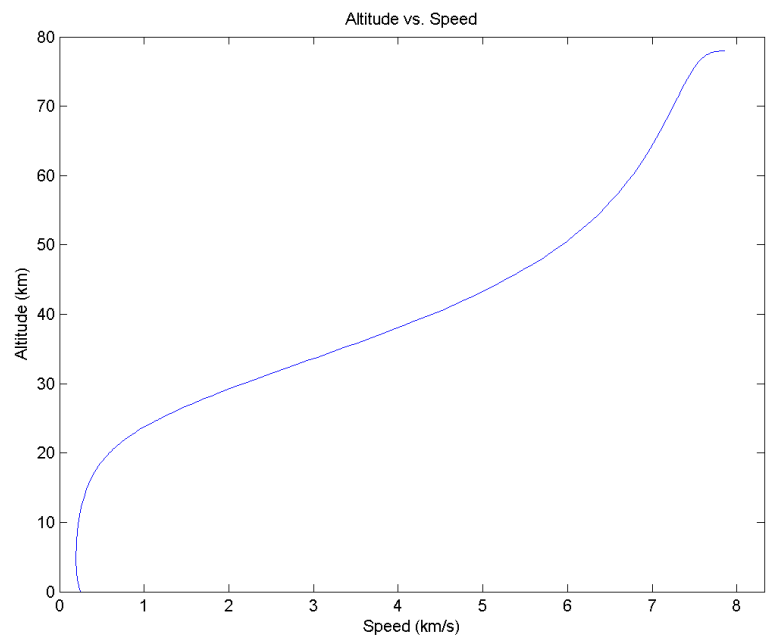

(a) Trajectory during reentry

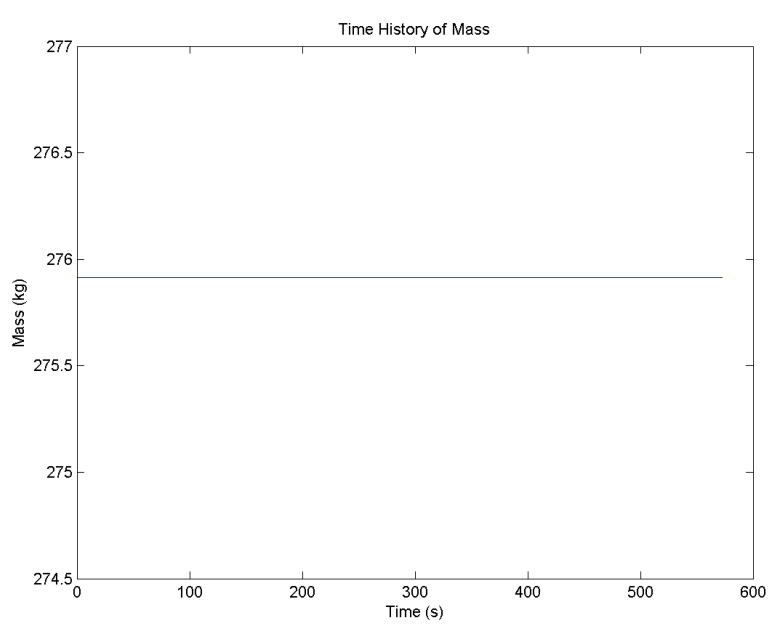

(b) Mass variation during reentry

\section{Figure 4.5: Molybdenum Cube}

\subsubsection{Silver}

The simulated reentry (plotted in Figure 4.6a) ended with the demise of the silver cube, at an altitude of $42.3 \mathrm{~km}$; DAS reentry analysis on the test cube predicts a demise altitude of $41.2 \mathrm{~km}$. Unlike the acrylic cube, with its extremely low heat of fusion, the silver cube has a significant resistance to ablation. Even when the cube reaches its melting temperature, it does not completely demise instantly, but over a period of several minutes, as seen in Figure 4.6b. This behavior is much more realistic than seen in the case of the acrylic cube, even using the same thermal model. 


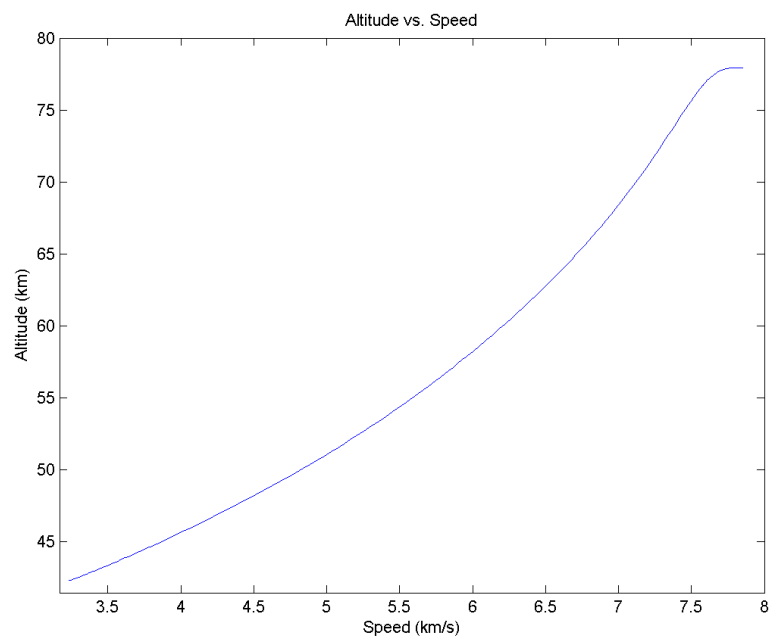

(a) Trajectory during reentry

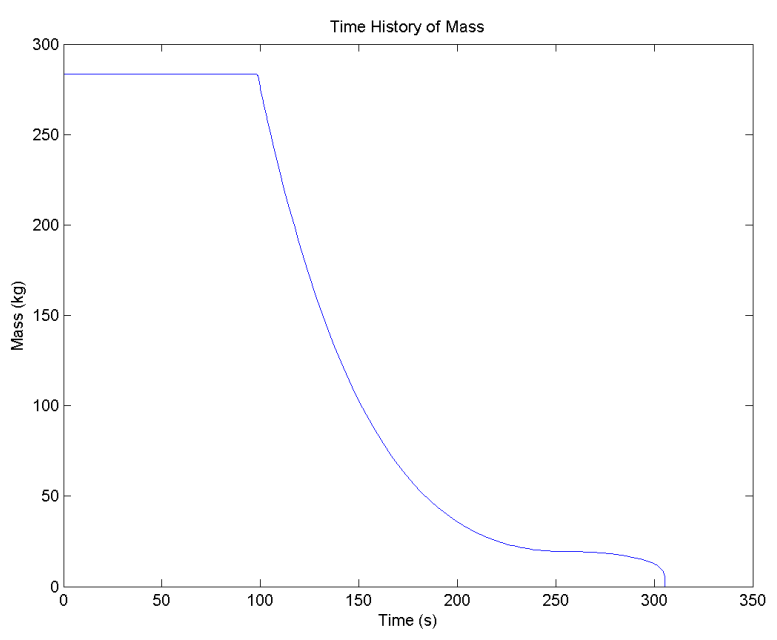

(b) Mass variation during reentry

Figure 4.6: Silver Cube

\subsection{Solid Cylinders}

The third test case is a solid cylinder of base radius $15 \mathrm{~cm}$ and side length $30 \mathrm{~cm}$, for a fineness ratio of 1 . The mass and demise total internal energy of each of the three cylinders being tested can be found in Table 4.6 (calculated using Equation 1.1, where $v$ is the spacecraft speed in $\mathrm{km} / \mathrm{s}, r$ is the radius in $\mathrm{km}, \mu$ is the standard gravitational

parameter in $\frac{k m^{3}}{s^{2}}$, and $e$ is the total specific energy in $\frac{M J}{k g}$ ). The total energy, calculated using Equation 4.1 (Equation 1.10 in [91]), of the body at its initial conditions (listed in Table 4.3) is -33.6 $\frac{M J}{k g}$ (hence -834 MJ for acrylic, -7479 MJ for silver, and -7285 MJ for molybdenum). Again using Equation 4.1 to calculate the energy at the surface (with zero velocity), we determine that the maximum energy available to be changed from potential and kinetic into heat is $717 \mathrm{MJ}$ for the acrylic, $6426 \mathrm{MJ}$ for the silver, and $6258 \mathrm{MJ}$ for the molybdenum. Again, though the demise internal energy of each cylinder is far below the available heat energy, demise is not guaranteed, since only a small portion of the heat goes into the body. 


\section{Table 4.6: Test Cylinder Mass and Demise Internal Energy}

\begin{tabular}{|c|c|c|}
\hline Material & Mass $(\mathrm{kg})$ & $h_{\text {ablation }}(\mathrm{MJ})$ \\
\hline Acrylic & 24.81 & 18.36 \\
Molybdenum & 216.70 & $209.1-322.2$ \\
Silver & 222.49 & $84.66-90.46$ \\
\hline
\end{tabular}

\subsubsection{Acrylic}

The acrylic cylinder demises during reentry, at an altitude of $74.0 \mathrm{~km}$, lower than either the acrylic cubes or spheres. The DAS simulation predicts the acrylic cylinder to demise at $73.3 \mathrm{~km}$. As with the acrylic sphere and cube, the demise of the cylinder is very fast once the melting temperature is reached, seen in Figure $4.7 \mathrm{~b}$ as a vertical line.

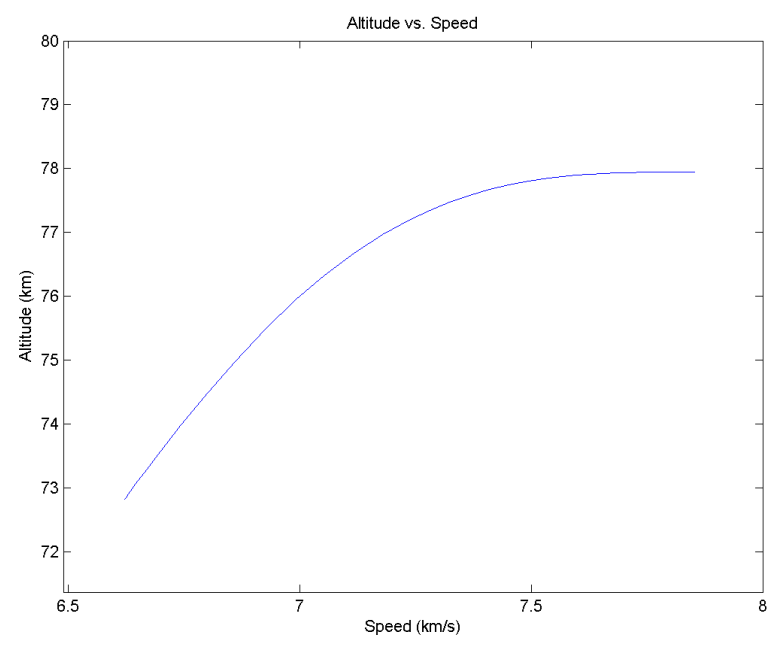

(a) Trajectory during reentry

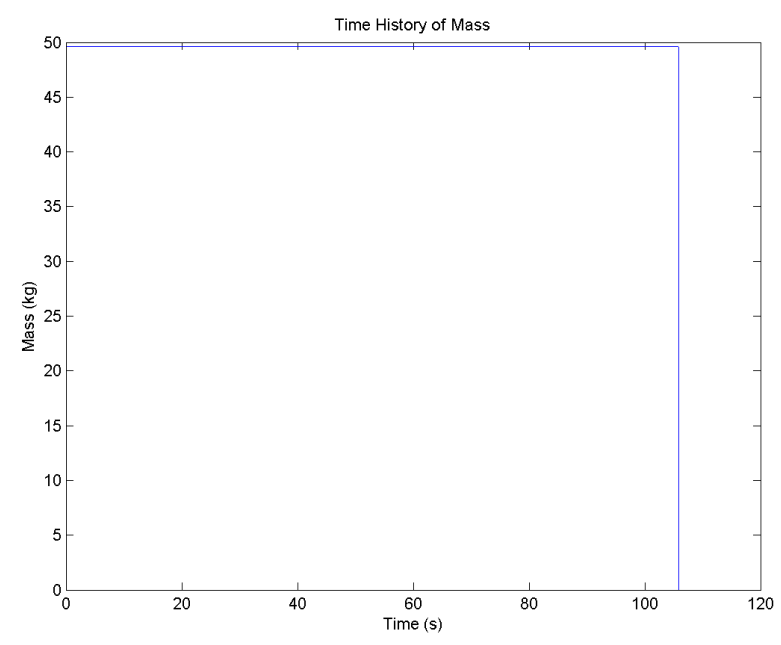

(b) Mass variation during reentry

Figure 4.7: Acrylic Cylinder

\subsubsection{Molybdenum}

As seen in Figure 4.8a, the molybdenum cylinder survives with its initial mass to impact the Earth's surface with a speed similar to the cube and sphere, despite the suggestion above that cylinders have higher heating rates and lower drag forces than spheres and 
cubes. The impact speed of the molybdenum cylinder is $317 \frac{\mathrm{m}}{\mathrm{s}}$, hence a kinetic energy of 10.89 MJ; DAS predicts an impact kinetic energy of $10.30 \mathrm{MJ}$, a difference of only $6 \%$. Since the total heat load experienced by the molybdenum cylinder was insufficient to raise it to its melting temperature, no mass was ablated (as seen by the horizontal line in Figure 4.8b).

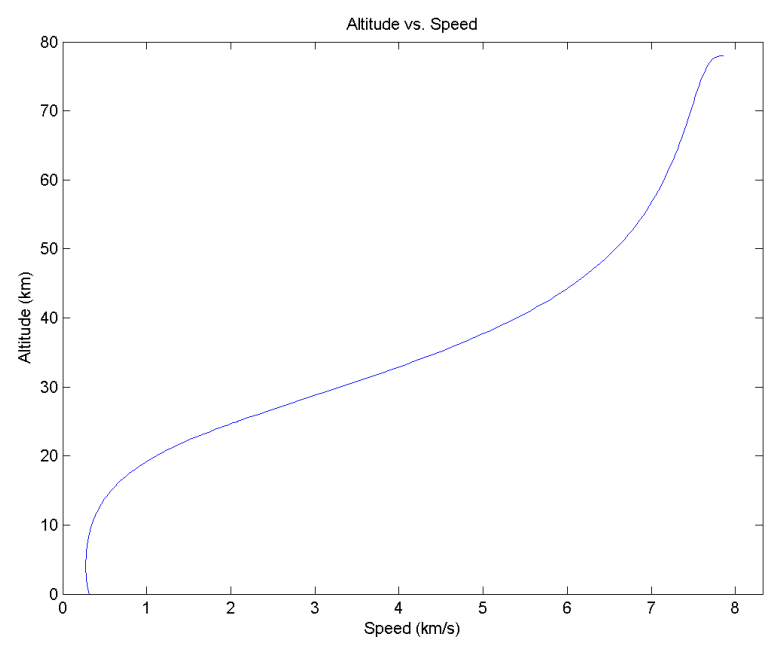

(a) Trajectory during reentry

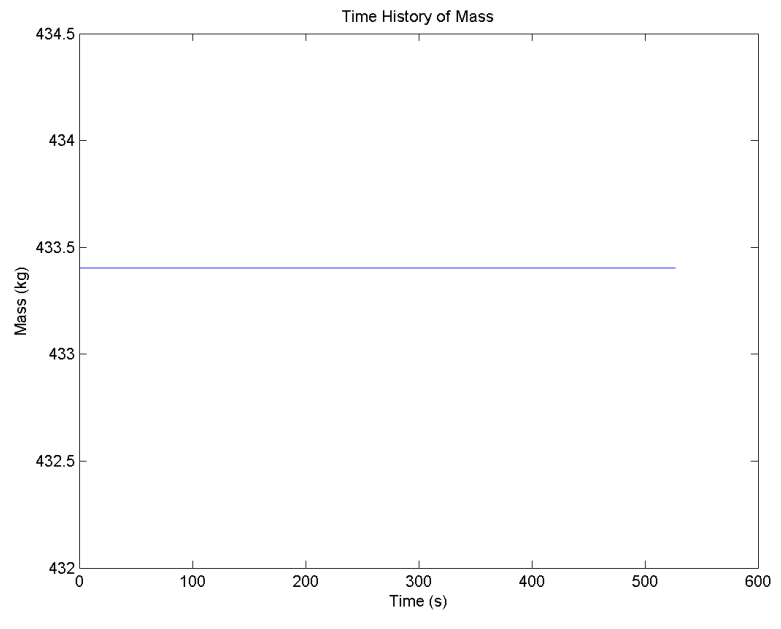

(b) Mass variation during reentry

Figure 4.8: Molybdenum Cylinder

\subsubsection{Silver}

The silver cylinder demised during the reentry simulation, at an altitude of $41.1 \mathrm{~km}$, as seen in Figure 4.9a. Reentry survivability analysis performed using DAS predicts demise of the silver cylinder at $43.3 \mathrm{~km}$. Unlike the acrylic cylinder, with its extremely low heat of fusion, the silver cylinder has a significant resistance to ablation. Even when the cylinder reaches its melting temperature, it does not completely demise instantly, but over a period of minutes, as seen in Figure 4.9b. This behavior is much more realistic than seen in the case of the acrylic cylinder, even using the same thermal model. 


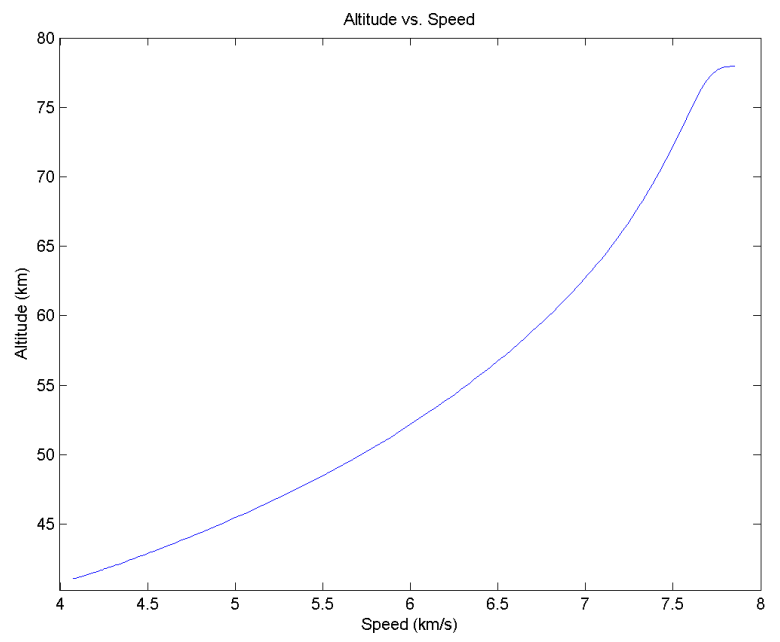

(a) Trajectory during reentry

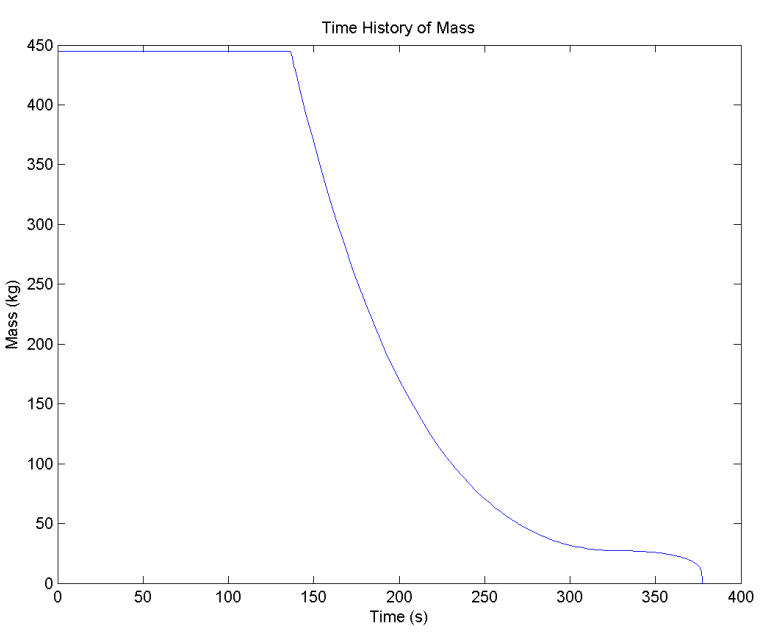

(b) Mass variation during reentry

Figure 4.9: Silver Cylinder 


\section{Chapter 5}

\section{Sensitivity Analysis}

Once the simulations using nominal parameters were complete, a Monte Carlo campaign was run, varying initial speed, initial altitude, freestream temperature, freestream density, and initial body temperature to assess their individual impact on downrange distance, final mass, and demise altitude (or impact speed, if the object did not demise during reentry). The maximum and minimum values for each parameter are listed in Table 5.1. Example plots will be presented for each shape and for two presently-described categories of materials: those that demise, and those that survive to impact the surface (no matter the final mass).

Table 5.1: Parameters for Sensitivity Analysis

\begin{tabular}{|c|c|c|c|}
\hline Quantity & Range & Nominal Value & Unit \\
\hline Initial Altitude & $75-81$ & 78 & $\mathrm{~km}$ \\
Initial Speed & $7.60-8.19$ & 7.85 & $\mathrm{~km} / \mathrm{s}$ \\
Freestream Temperature (Scaling) & $0.75-1.25$ & 1 & {[]} \\
Freestream Density (Scaling) & $0.75-1.25$ & 1 & {[]} \\
Initial Temperature & $150-600$ & 300 & $\mathrm{~K}$ \\
\hline
\end{tabular}

\subsection{Initial Speed}

The first variable to be analyzed is the initial speed, which will be varied from 7.60 to $8.19 \frac{\mathrm{km}}{\mathrm{s}}$ (or approximately -3 to +4 percent of the nominal speed) over 500 simulated trajectories, and plotted against the final properties of interest as mentioned above: downrange distance, final mass, final altitude, and final impact speed. 


\subsubsection{Downrange Distance}

Downrange distance is calculated as the length of the ground track from the initial position to either the demise of the body, or its impact with the surface. The effect of the initial speed on downrange distance can be estimated, using Equation 5.1 as approximately 6 kilometers per meter per second.

$$
\frac{\partial D R}{\partial v_{0}} \approx \frac{\left.D R\right|_{v_{\max }}-\left.D R\right|_{v_{\min }}}{v_{\max }-v_{\min }}
$$

\subsubsection{Sphere}

The molybdenum and silver spheres both feature a trend toward shorter downrange distances to impact and demise, in Figures 5.1a and 5.1b respectively, as the initial speed increases. The initial speed is changed, at a constant flight path angle, causing the radial component of the velocity to become greater as the total speed increases, the largest contributor to the shorter downrange distance. In the case of silver, this is partially due to the increased heating rate experienced at the beginning of the trajectory at the higher speeds.

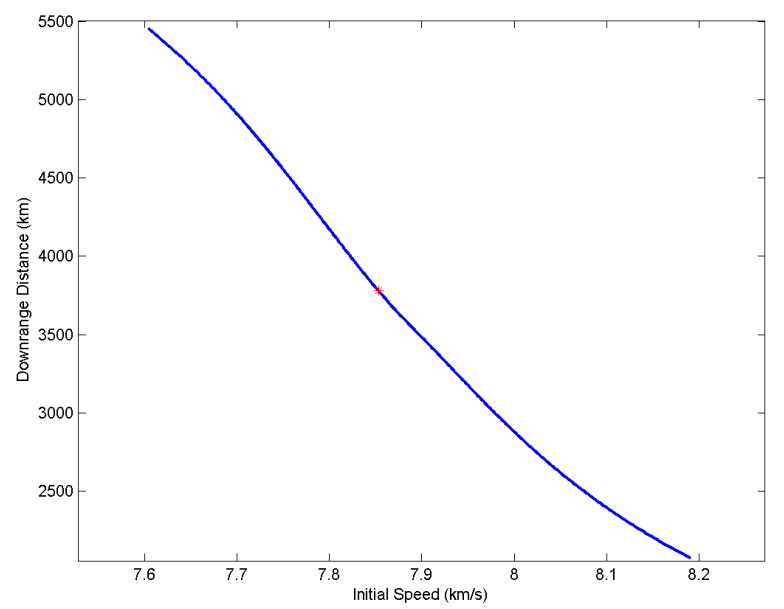

(a) Molybdenum

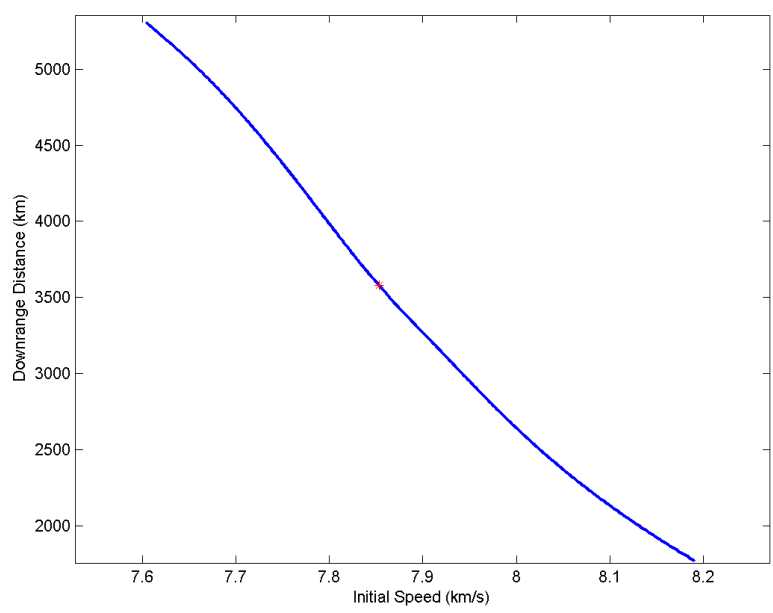

(b) Silver

Figure 5.1: Downrange Distance vs. Initial Speed (Sphere) 


\subsubsection{Cube}

Cubes tended to follow the same trend as the spheres in the section above, with the downrange distance decreasing with increasing speed for both molybdenum and silver (see Figures 5.2a and 5.2b, respectively).

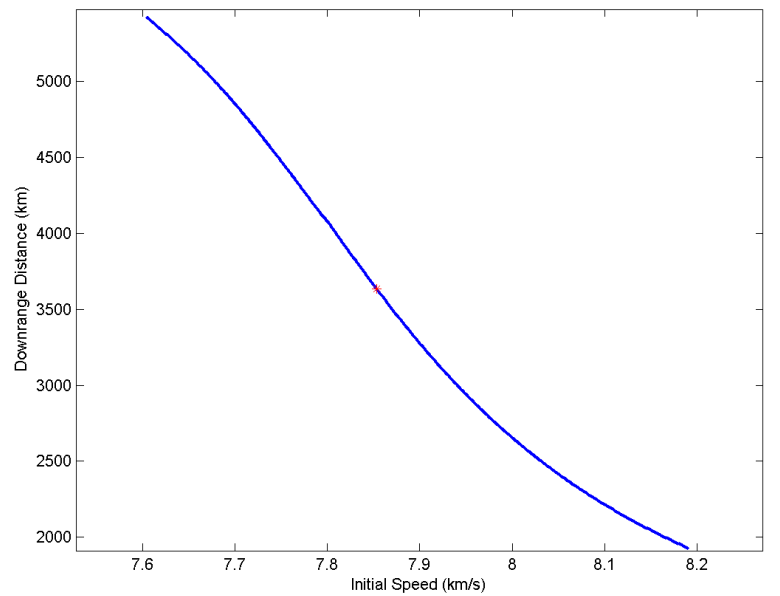

(a) Molybdenum

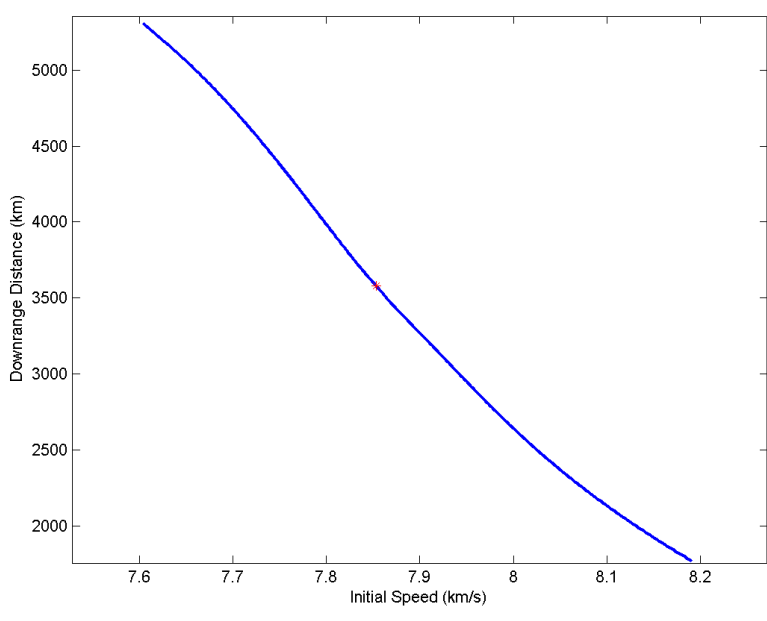

(b) Silver

Figure 5.2: Downrange Distance vs. Initial Speed (Cube)

\subsubsection{Cylinder}

Both materials in this analysis showed similar trends (see Figures 5.3a and 5.3b for molybdenum and silver, respectively) as the cubes and spheres, with increased initial speeds causing decreased final downrange distances. 


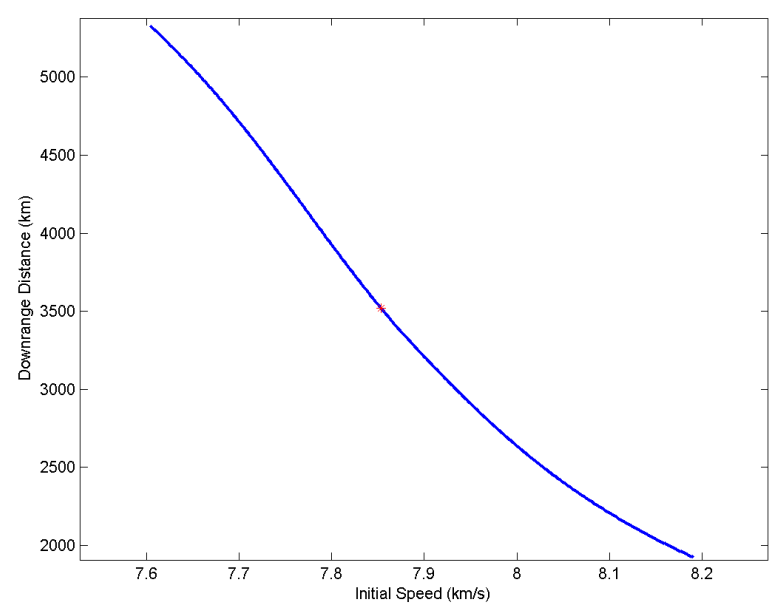

(a) Molybdenum

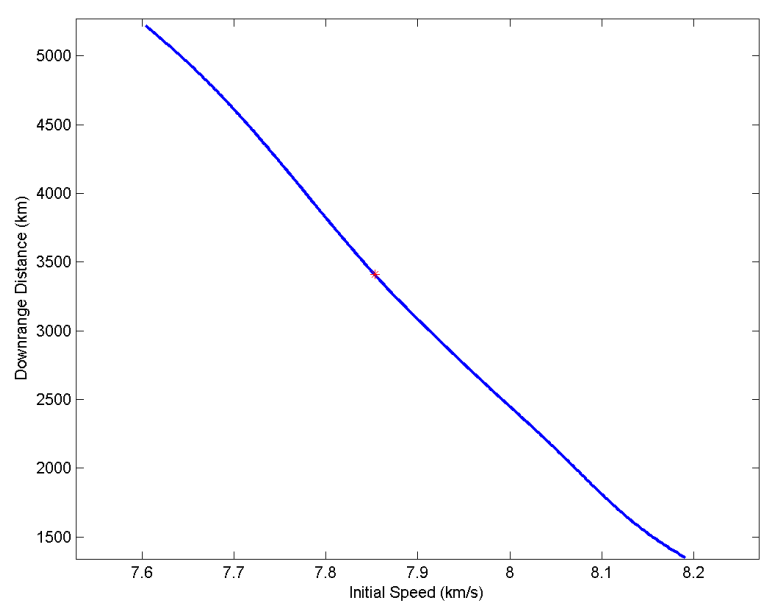

(b) Silver

Figure 5.3: Downrange Distance vs. Initial Speed (Cylinder)

\subsubsection{Final Mass}

The final mass is, as expected, the mass at the end of each simulated trajectory: if the object demises, the mass is zero (and the following plot appears as a horizontal line at zero); if the object survives with no ablation, the mass is the same as its initial mass on-orbit (and the following plot would appear as a horizontal line at that value). Since no molybdenum sample lost mass during the simulated trajectories and every silver sample demised, it appears that (for these two classes of materials) the final mass is insensitive to small (here, on the order of 3-4 percent) changes in initial speed.

\subsubsection{Sphere}

Figures 5.4a and 5.4b appear similar (as horizontal lines), all the masses are identical for a material: the molybdenum samples did not lose any mass during the simulation, while all the silver samples demised before impact. 


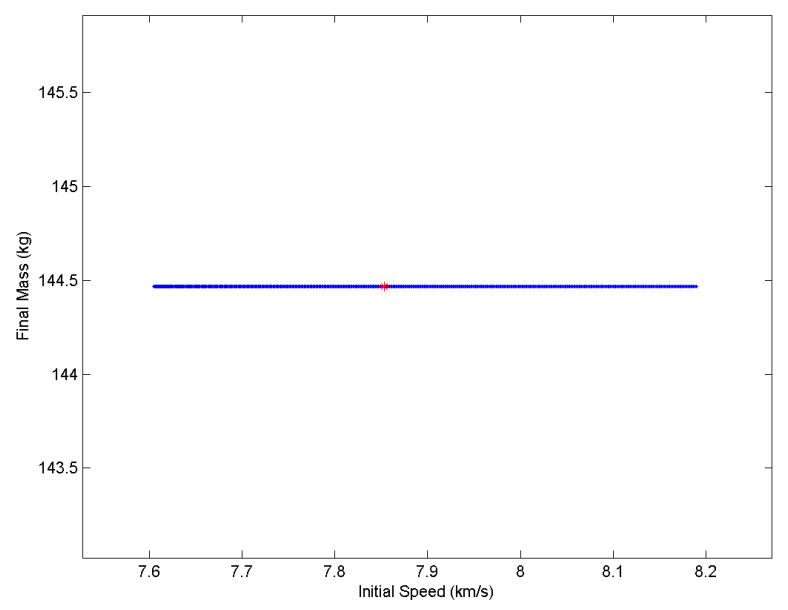

(a) Molybdenum

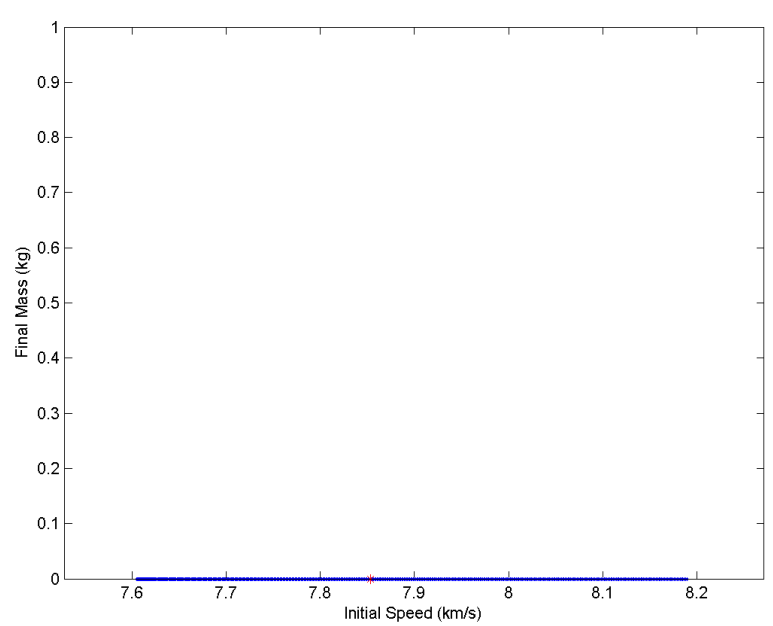

(b) Silver

Figure 5.4: Final Mass vs. Initial Speed (Sphere)

\subsubsection{Cube}

As with the spheres, the cubes' masses did not vary with initial speed for these materials: the molybdenum cubes lost no mass, and every silver cube demised before impact (seen as horizontal lines in Figures 5.5a and 5.5b, respectively).

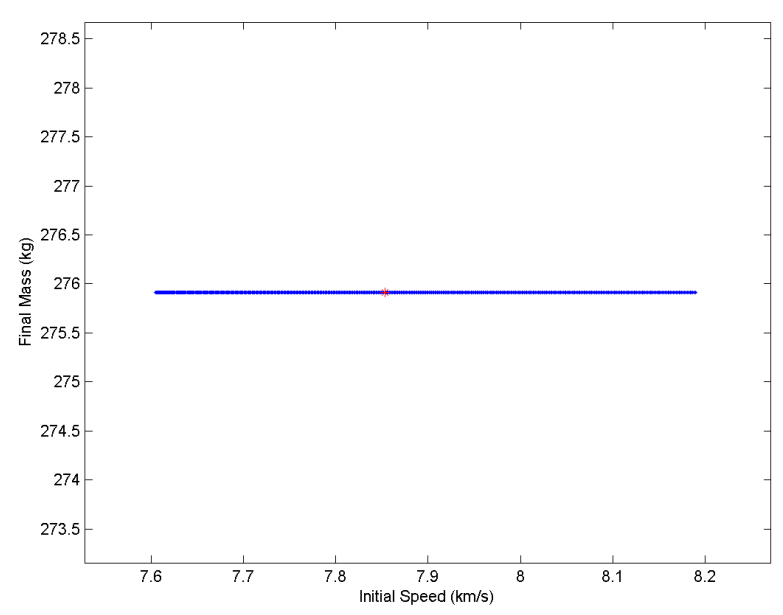

(a) Molybdenum

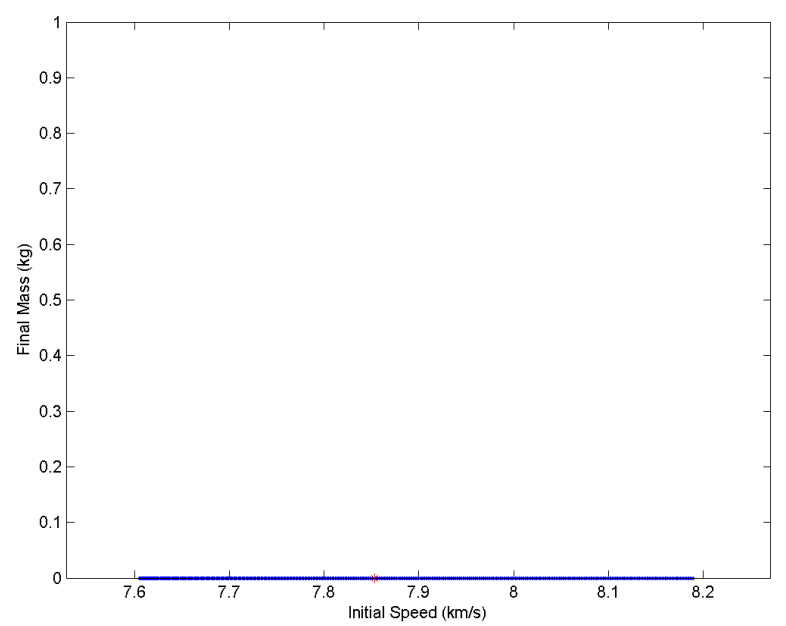

(b) Silver

Figure 5.5: Final Mass vs. Initial Speed (Cube) 


\subsubsection{Cylinder}

Cylinder final masses also appear to be insensitive to the small changes in initial speed simulated here. The horizontal lines in Figures 5.6a and 5.6b show that the molybdenum samples remain at the initial mass and the silver samples all demise, irrespective of initial speed.

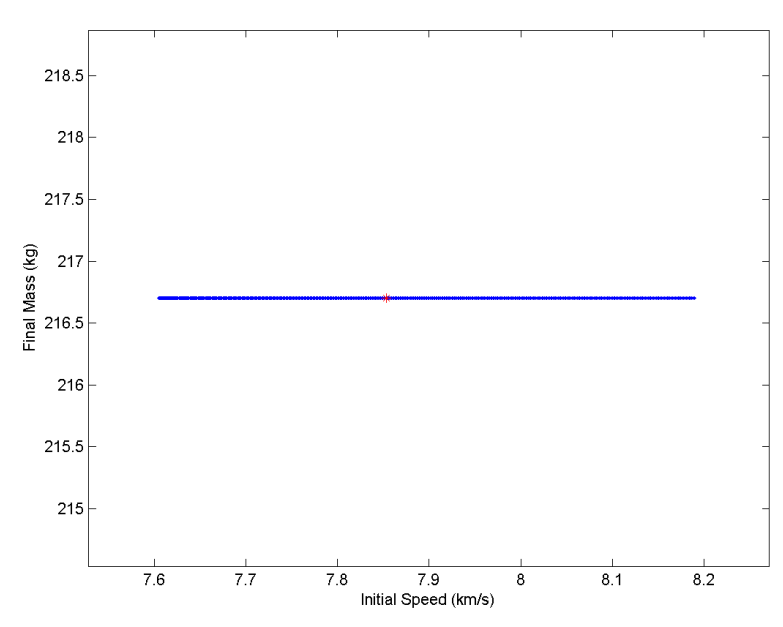

(a) Molybdenum

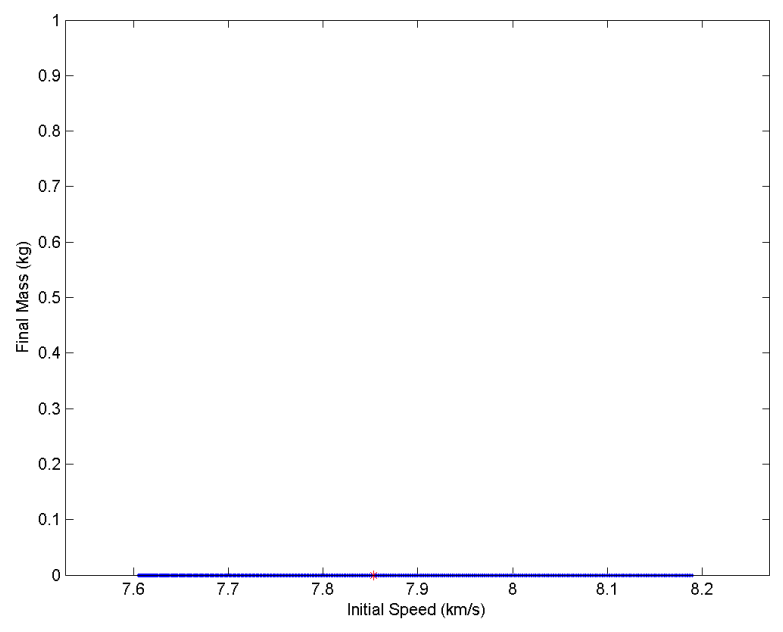

(b) Silver

Figure 5.6: Final Mass vs. Initial Speed (Cylinder)

\subsubsection{Final Speed}

The final speed is the speed at either the demise of the reentry body, or at the body's impact with the Earth's surface. The impact speed of the molybdenum samples was nearly insensitive to the initial speed, changing by less than $0.1 \%$. The silver samples, however, varied by as much as $50 \%$ with only a $4 \%$ change in initial speed.

\subsubsection{Sphere}

The trend in Figure 5.7a indicates that the initial speed is damped out by atmospheric drag by the time the sample reaches the surface. In contrast, Figure $5.7 \mathrm{~b}$ shows that samples that demise can have highly varying final speeds. The final speed of the silver spheres is related to the heating rate experienced in the upper atmosphere, which in- 
creases with the cube of initial speed; the drag increases with the squared speed, having a lesser effect on the final speed.

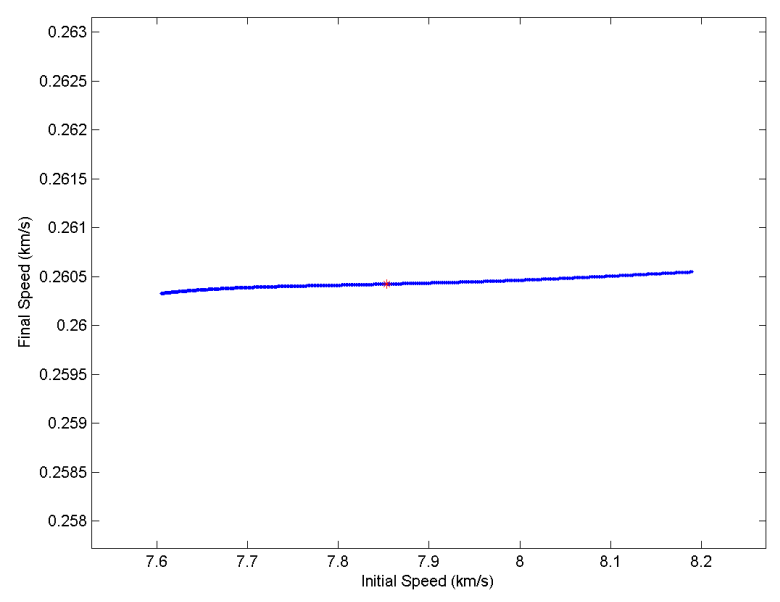

(a) Molybdenum

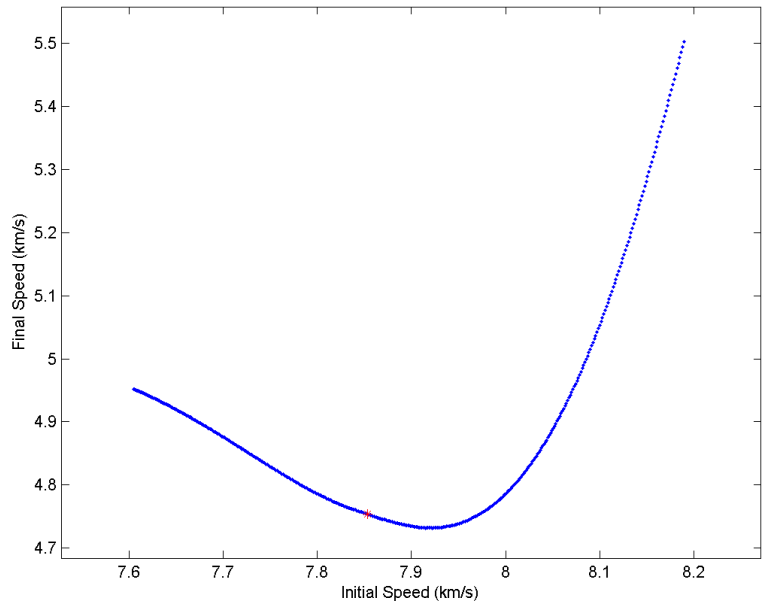

(b) Silver

\section{Figure 5.7: Final Speed vs. Initial Speed (Sphere)}

\subsubsection{Cube}

As discussed above, the final speed of the molybdenum samples is nearly invariant of initial speed(see nearly horizontal line in Figure 5.8a). The silver cubes, as with the spheres, demonstrated a strong relationship between initial speed and final speed (see Figure 5.8b). 


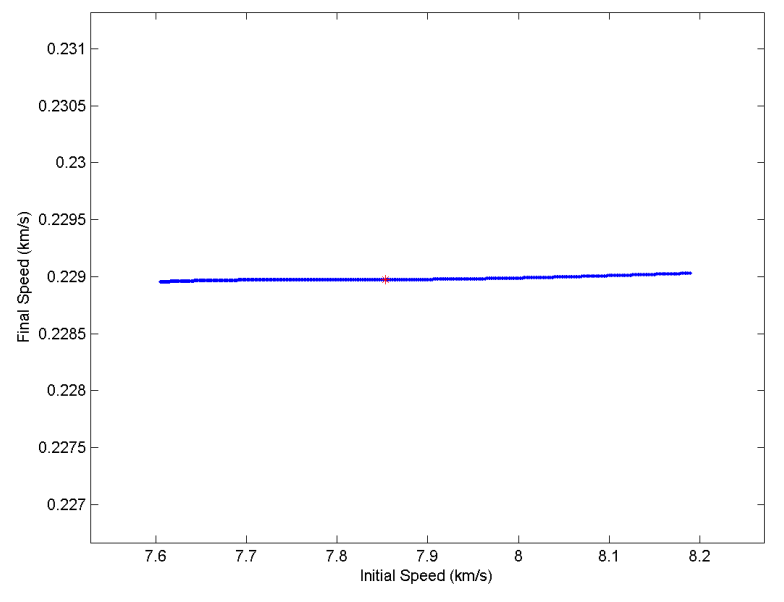

(a) Molybdenum

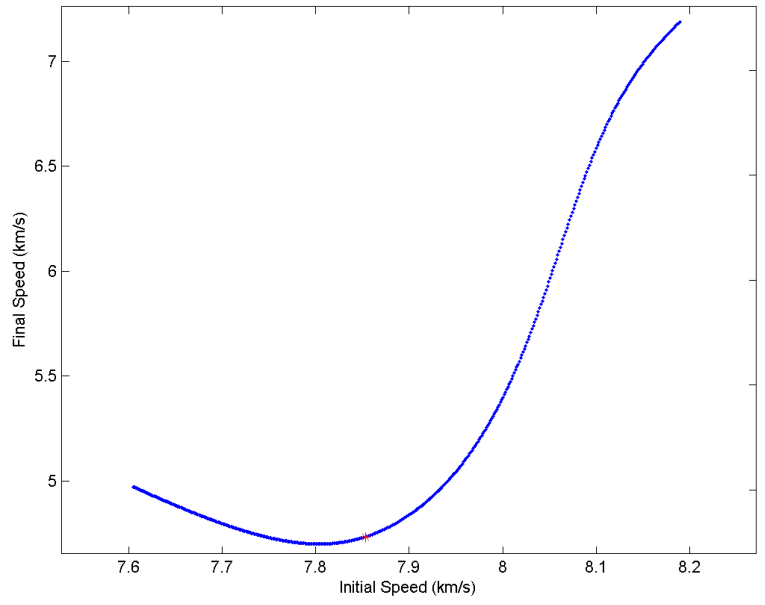

(b) Silver

Figure 5.8: Final Speed vs. Initial Speed (Cube)

\subsubsection{Cylinder}

Again, the final speed of the molybdenum samples varies minimally with initial speed (seen in Figure 5.9a), the variation of which is likely damped out by atmospheric drag. The final speed of the silver samples varies by up to $50 \%$ compared to the nominal initial speed, as in Figure 5.9b.

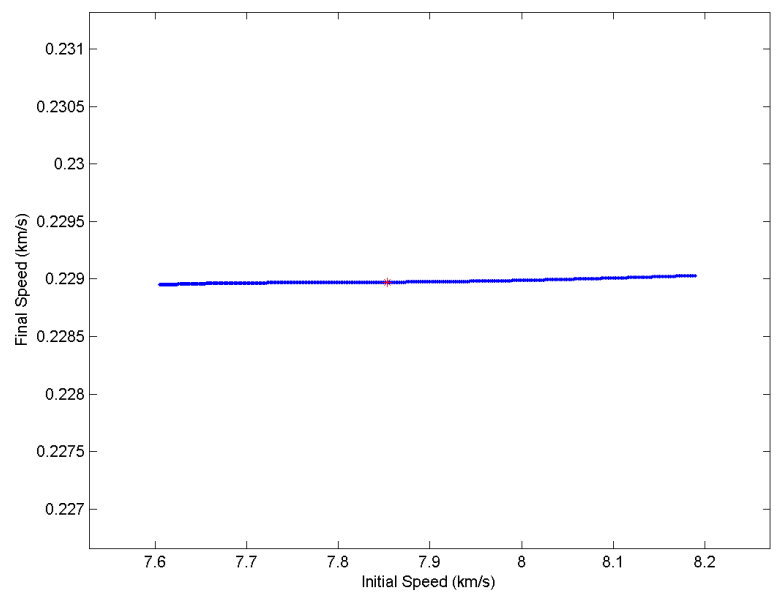

(a) Molybdenum

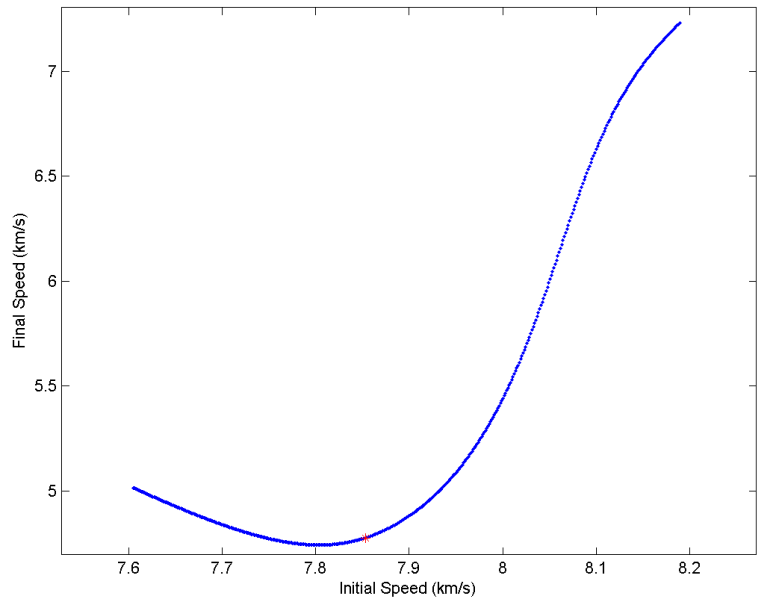

(b) Silver

Figure 5.9: Final Speed vs. Initial Speed (Cylinder) 


\subsubsection{Demise Altitude}

Demise altitude is the altitude at which the mass of the reentry object reaches zero. If the object does not reach zero mass, the impact altitude (identically zero) is instead plotted here. Molybdenum samples all survived to impact regardless of initial speed in this simulation; all silver samples demised, with demise altitude depending strongly on initial speed - a $4 \%$ increase in speed corresponded to a $10 \%$ change in demise altitude.

\subsubsection{Sphere}

Molybdenum was chosen as one of the sample materials since its physical properties caused all its mass to survive until impact with the surface (seen as a horizontal line at zero in Figure 5.10a). The demise altitude for silver varied as much as $10 \%$ from the nominal demise altitude, and increased dramatically with increasing initial speed (as seen in Figure 5.10b).

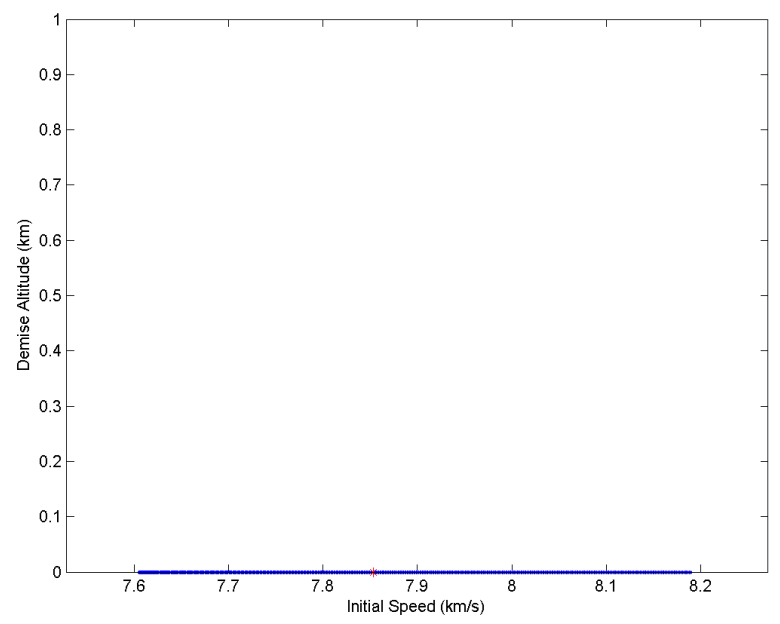

(a) Molybdenum

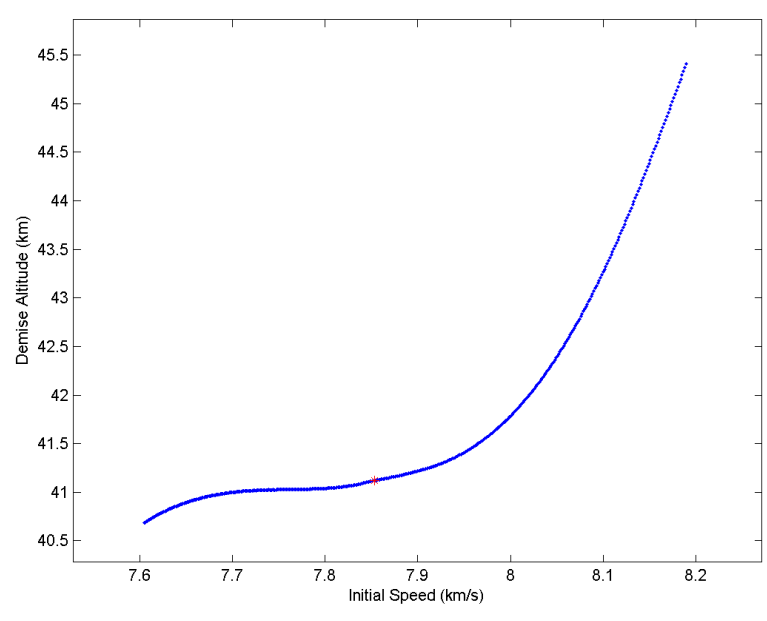

(b) Silver

Figure 5.10: Demise Altitude vs. Initial Speed (Sphere)

\subsubsection{Cube}

Every molybdenum cube survives reentry to impact the surface, as seen in Figure 5.11a as a horizontal line at zero. Silver cubes have demise altitudes that vary by up to $10 \%$ of 
the nominal altitude, with the demise altitude increasing with initial speed (see Figure $5.11 b)$.

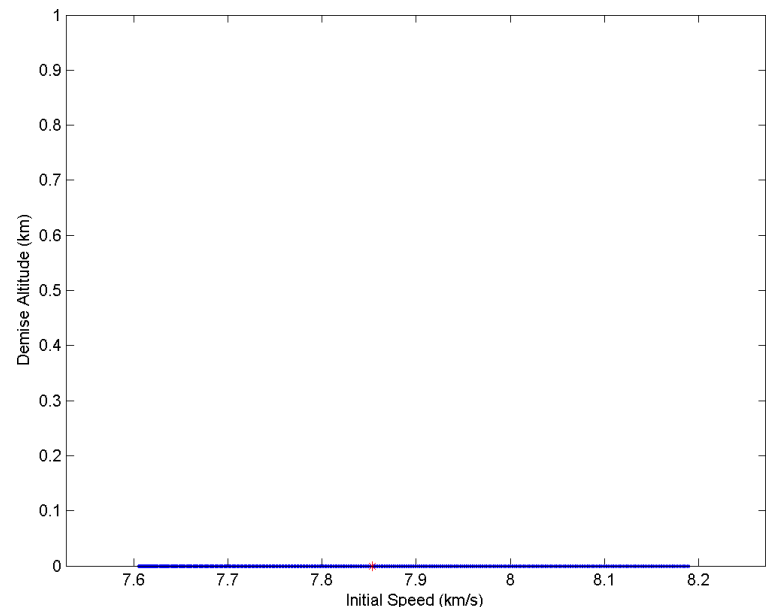

(a) Molybdenum

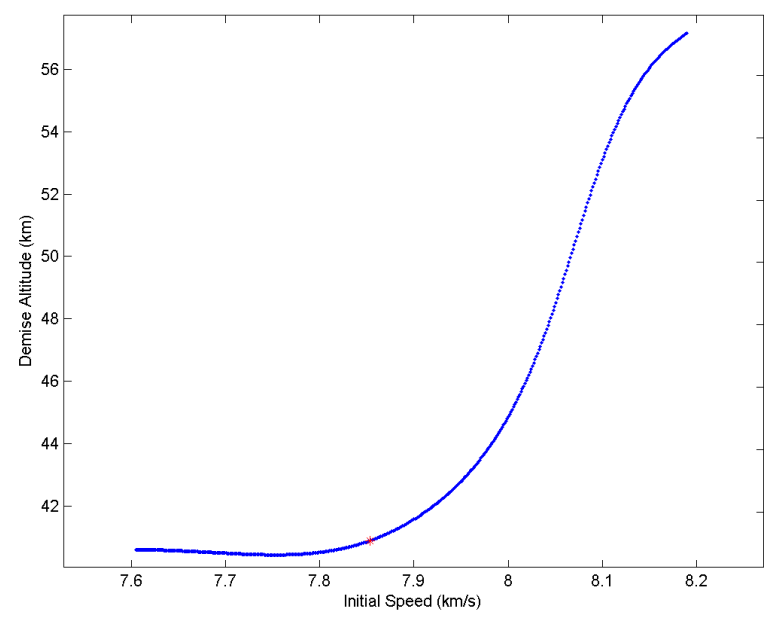

(b) Silver

Figure 5.11: Demise Altitude vs. Initial Speed (Cube)

\subsubsection{Cylinder}

Cylinders had the same behavior as the cubes and spheres: molybdenum samples all survived reentry, and all silver samples demised, with the altitude increasing with initial speed (see Figures 5.12a and 5.12b, respectively).

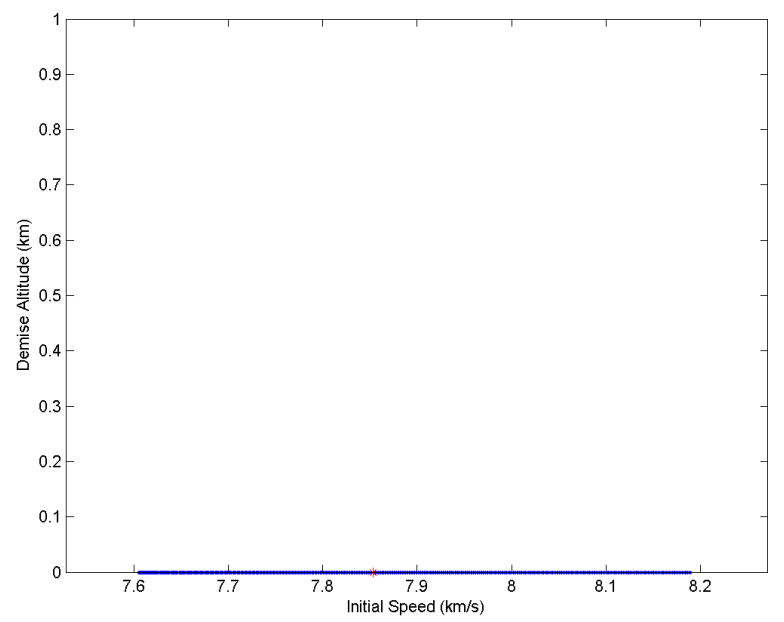

(a) Molybdenum

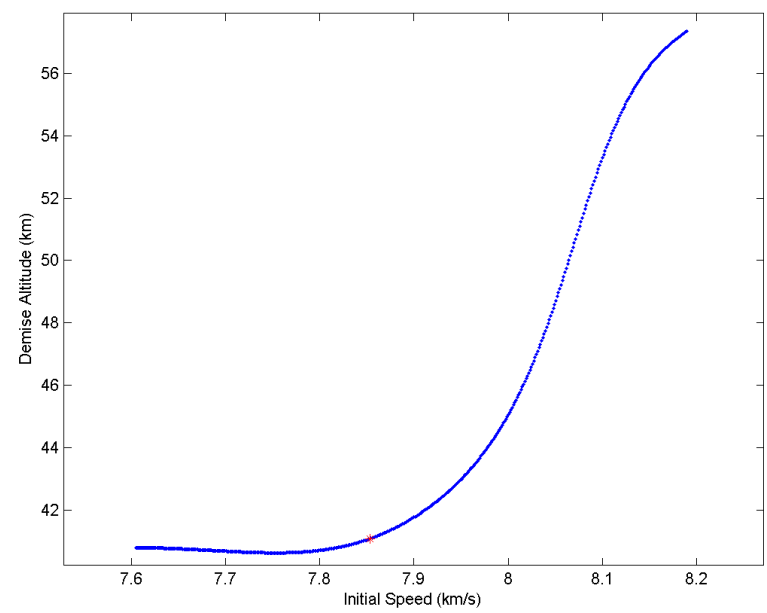

(b) Silver

Figure 5.12: Demise Altitude vs. Initial Speed (Cylinder) 


\subsection{Initial Altitude}

The second variable to be analyzed is the initial altitude, which will be varied from 75 to $81 \mathrm{~km}$ (or approximately -4 to +4 percent of the nominal speed) over 500 simulated trajectories, and plotted against the final properties of interest as mentioned above: downrange distance, final mass, final altitude, and final impact speed.

\subsubsection{Downrange Distance}

Downrange distance is calculated as the length of the ground track from the initial position to either the demise of the body, or its impact with the surface. The effect of the initial altitude on downrange distance can be estimated, using Equation 5.2 as approximately 40-50 kilometers of downrange distance per kilometer of initial altitude.

$$
\frac{\partial D R}{\partial h_{0}} \approx \frac{\left.D R\right|_{h_{\max }}-\left.D R\right|_{h_{\min }}}{h_{\max }-h_{\min }}
$$

\subsubsection{Sphere}

Downrange distance varies nearly linearly with initial altitude for both molybdenum and silver spheres (see Figures 5.13a and 5.13b, respectively). This increase can be partially explained by the lower drag and heating rates experienced as the initial altitude increases. 


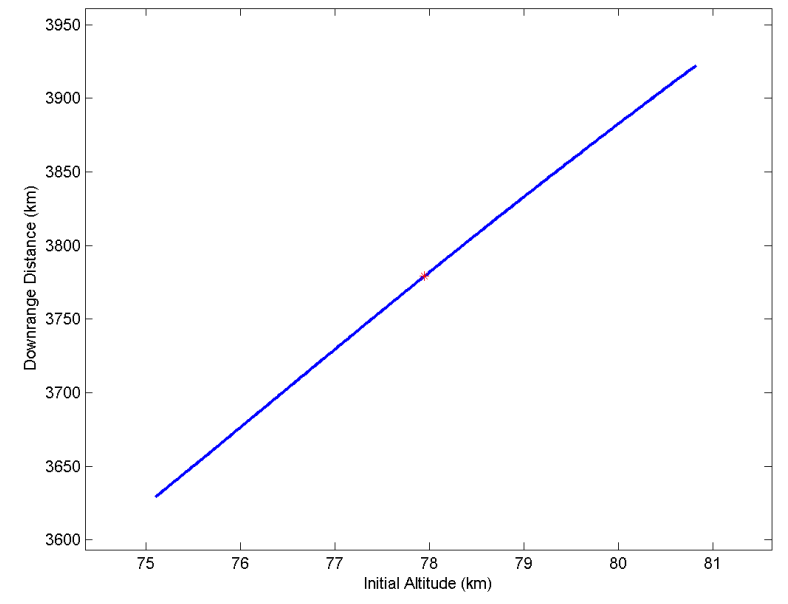

(a) Molybdenum

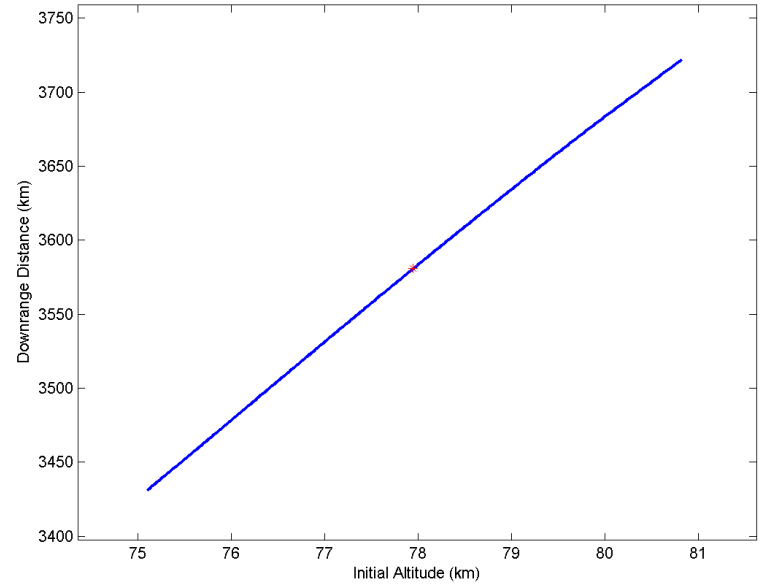

(b) Silver

Figure 5.13: Downrange Distance vs. Initial Altitude (Sphere)

\subsubsection{Cube}

Cubes tended to follow the same trend as the spheres in the section above, with the downrange distance increasing with increasing altitude for both molybdenum and silver. The sensitivity of downrange distance to initial altitude is greater in cubes than in spheres (see greater slopes in Figures 5.14a and 5.14b than in Figures 5.13a and 5.13b).

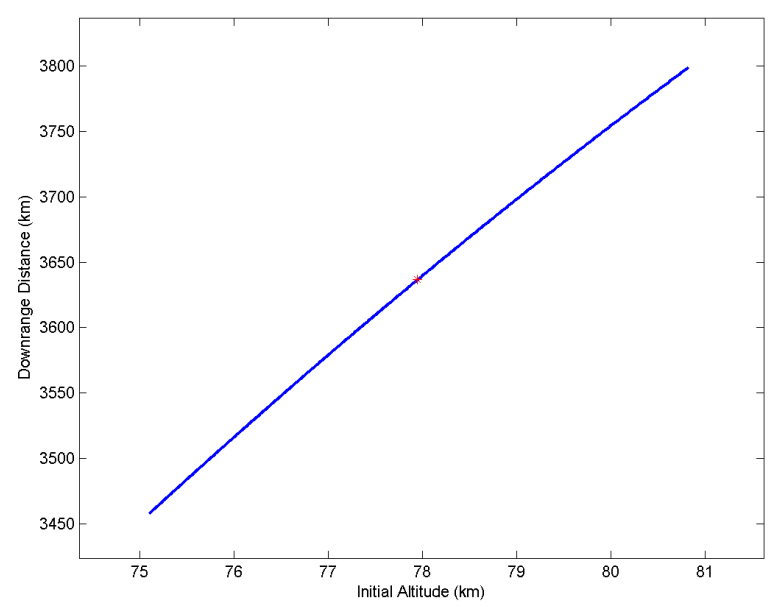

(a) Molybdenum

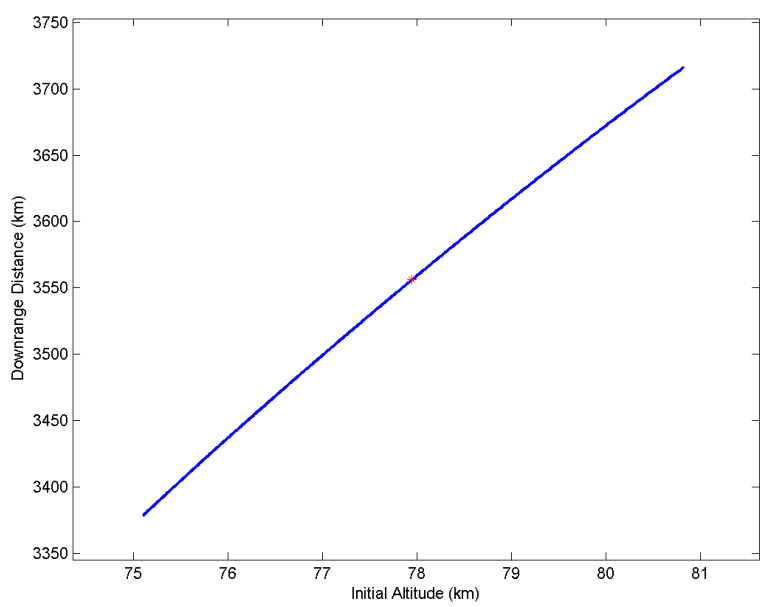

(b) Silver

Figure 5.14: Downrange Distance vs. Initial Altitude (Cube) 


\subsubsection{Cylinder}

Both materials in this analysis showed similar trends (see Figures 5.15a and 5.15b for molybdenum and silver, respectively) as the cubes and spheres, with increased initial altitudes causing increased final downrange distances.

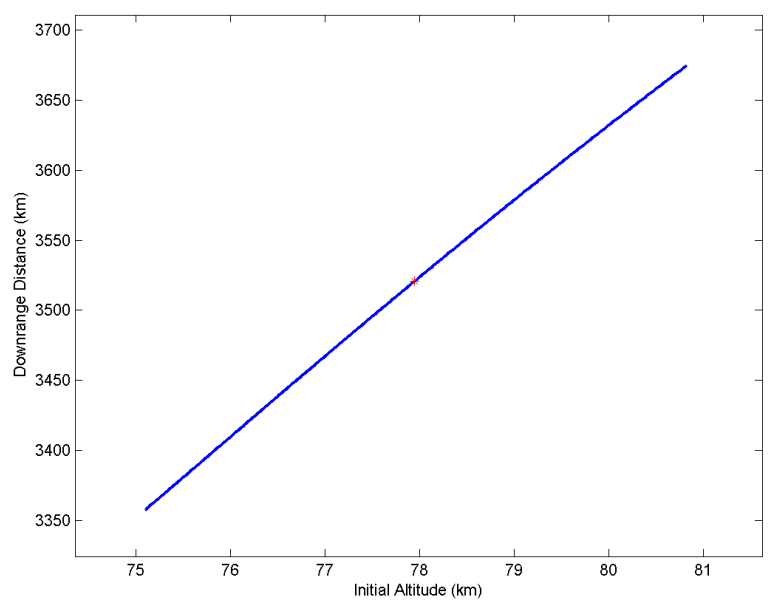

(a) Molybdenum

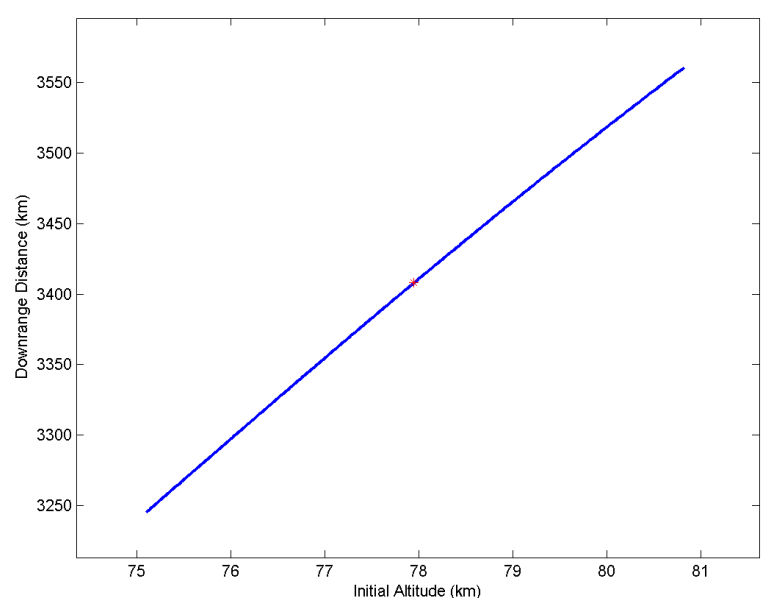

(b) Silver

Figure 5.15: Downrange Distance vs. Initial Altitude (Cylinder)

\subsubsection{Final Mass}

The final mass is, as expected, the mass at the end of each simulated trajectory: if the object demises, the mass is zero (and the following plot appears as a horizontal line at zero); if the object survives with no ablation, the mass is the same as its initial mass on-orbit (and the following plot would appear as a horizontal line at that value).

\subsubsection{Sphere}

Figures 5.16a and 5.16b indicate similar results, with all the masses identical for a material: the molybdenum samples did not lose any mass during the simulation, while all the silver samples demised before impact. 


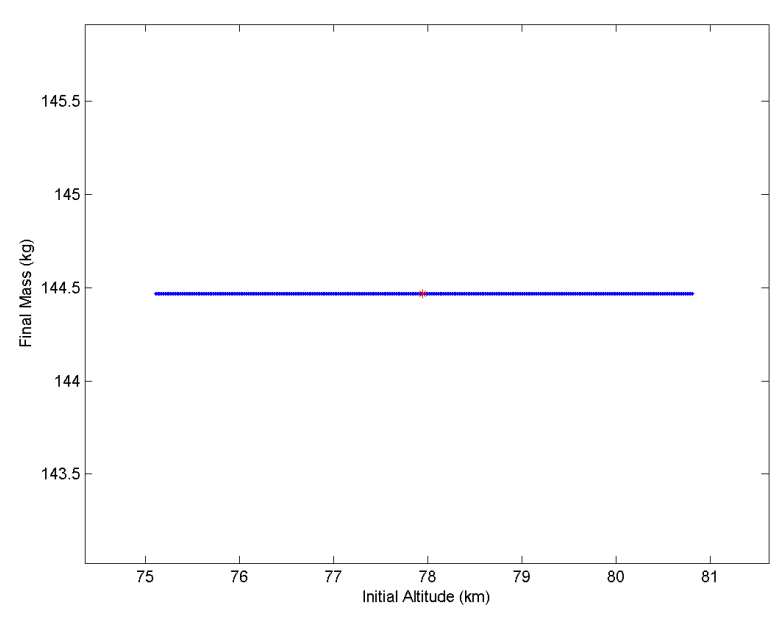

(a) Molybdenum

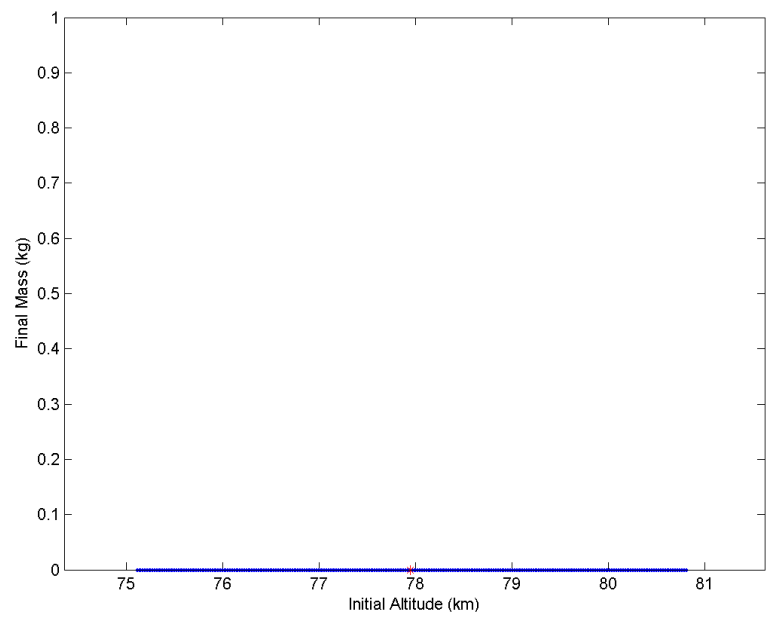

(b) Silver

Figure 5.16: Final Mass vs. Initial Altitude (Sphere)

\subsubsection{Cube}

As with the spheres, the cubes' masses did not vary with initial altitude for these materials: the molybdenum cubes lost no mass, and every silver cube demised before impact (seen as horizontal lines in Figures 5.17a and 5.17b, respectively).

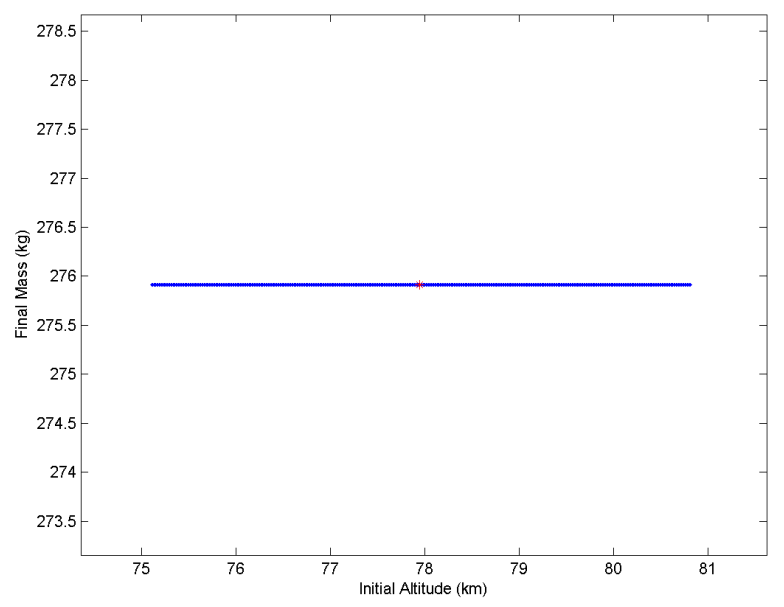

(a) Molybdenum

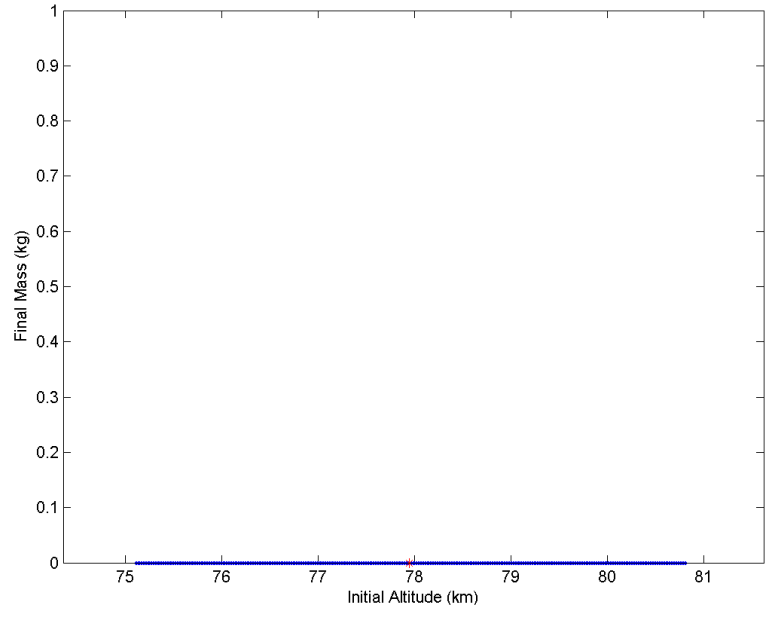

(b) Silver

Figure 5.17: Final Mass vs. Initial Speed (Cube) 


\subsubsection{Cylinder}

Cylinder final masses also appear to be insensitive to the small changes in initial altitude simulated here. The horizontal lines in Figures 5.18a and 5.18b show that the molybdenum samples remain at the initial mass and the silver samples all demise, irrespective of initial altitude.

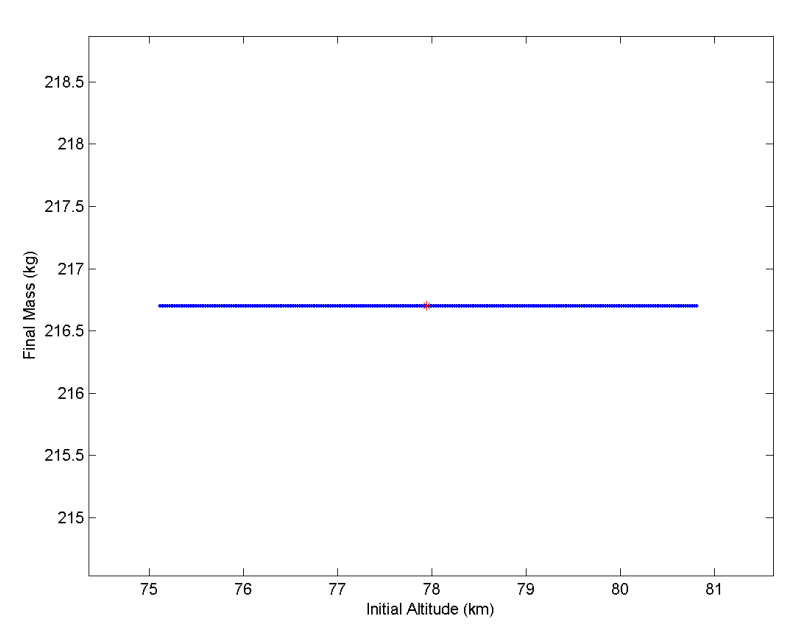

(a) Molybdenum

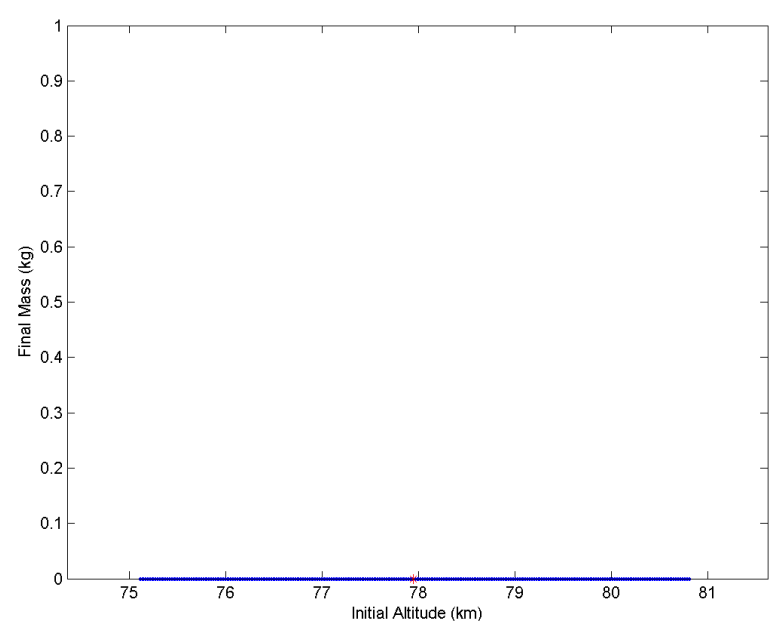

(b) Silver

\section{Figure 5.18: Final Mass vs. Initial Speed (Cylinder)}

\subsubsection{Final Speed}

The final speed is the speed at either the demise of the reentry body, or at the body's impact with the Earth's surface. The impact speed of the molybdenum samples was insensitive to the initial altitude. The silver samples also varied only slightly with altitude, changing by only about $1 \%$ at most.

\subsubsection{Sphere}

The trend in Figure 5.19a indicates that any speed increase from having higher initial gravitational potential energy is damped out by atmospheric drag by the time the sample reaches the surface. Figure 5.19b shows that samples that demise can have varying final speeds. 


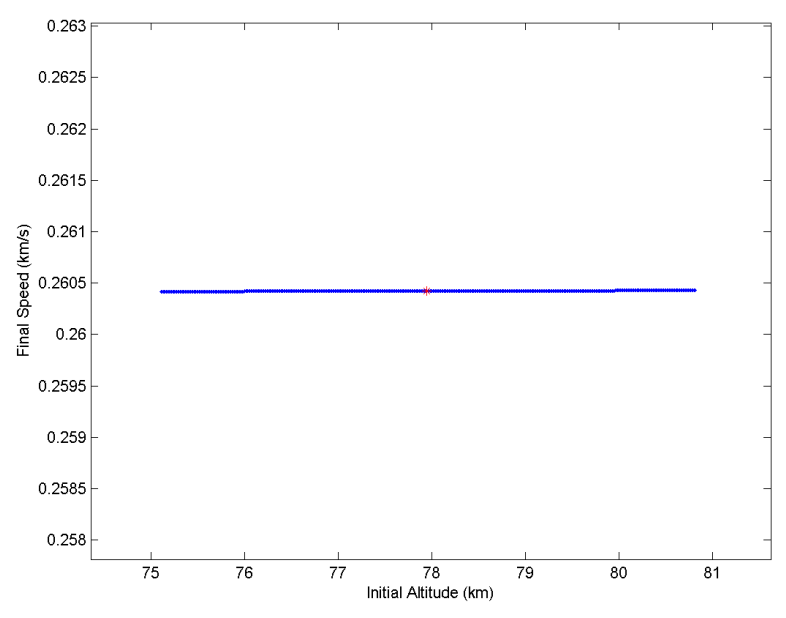

(a) Molybdenum

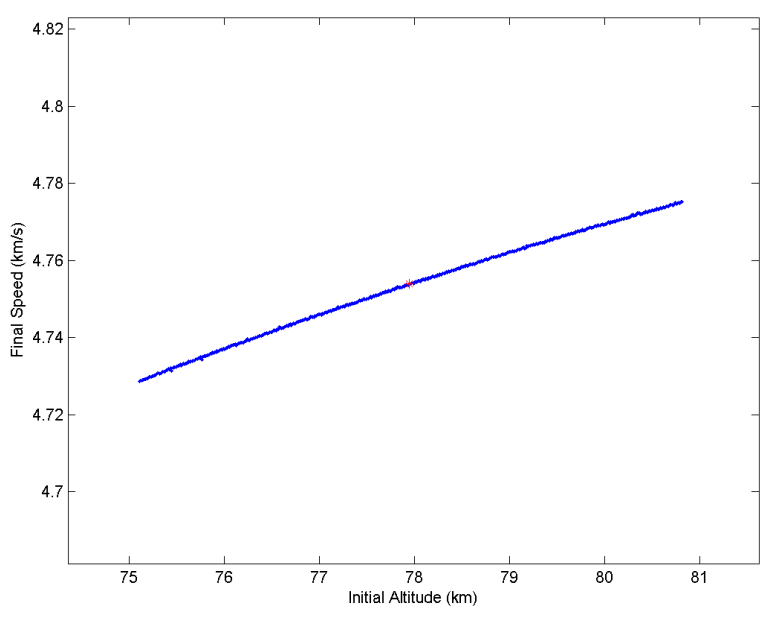

(b) Silver

Figure 5.19: Final Speed vs. Initial Altitude (Sphere)

\subsubsection{Cube}

As discussed above, the final speed of the molybdenum samples is nearly invariant of initial altitude (see nearly horizontal line in Figure 5.20a). The silver cubes, as with the spheres, demonstrated a nearly linear relationship between initial altitude and final speed (see Figure 5.20b).

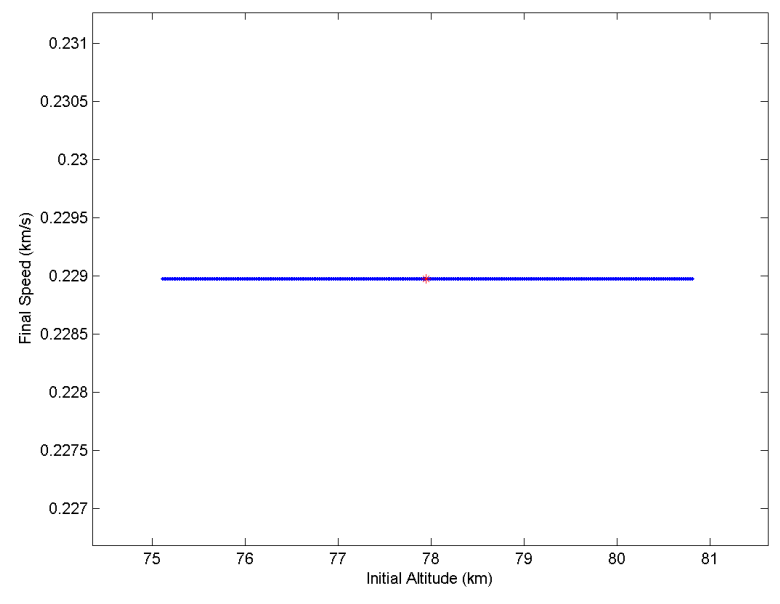

(a) Molybdenum

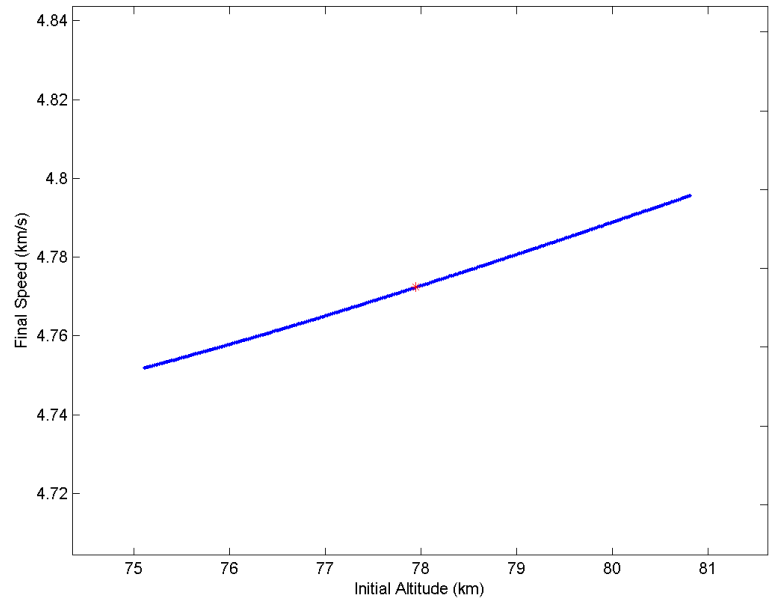

(b) Silver

Figure 5.20: Final Speed vs. Initial Altitude (Cube) 


\subsubsection{Cylinder}

Again, the final speed of the molybdenum samples varies minimally with initial altitude (seen in Figure 5.21a), the variation of which is likely damped out by atmospheric drag. The final speed of the silver samples varies by up to $1 \%$ compared to the nominal initial altitude, as in Figure 5.21b.

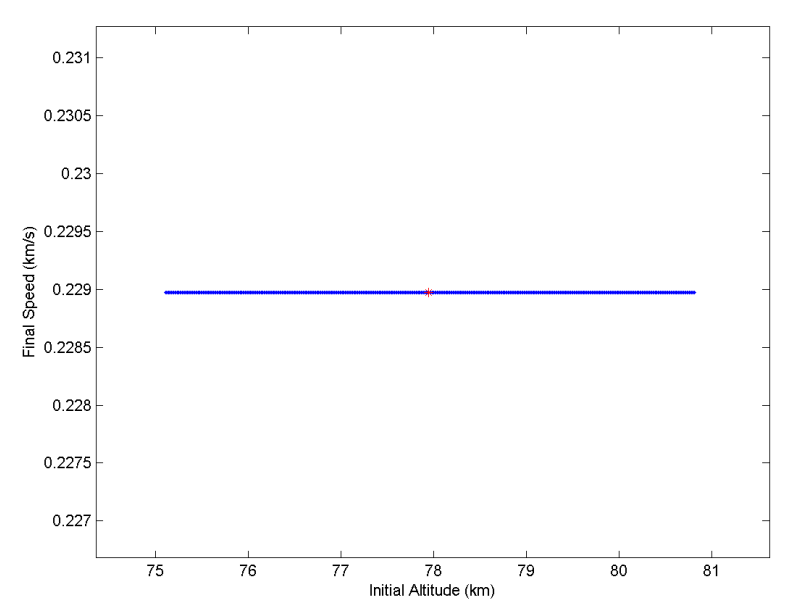

(a) Molybdenum

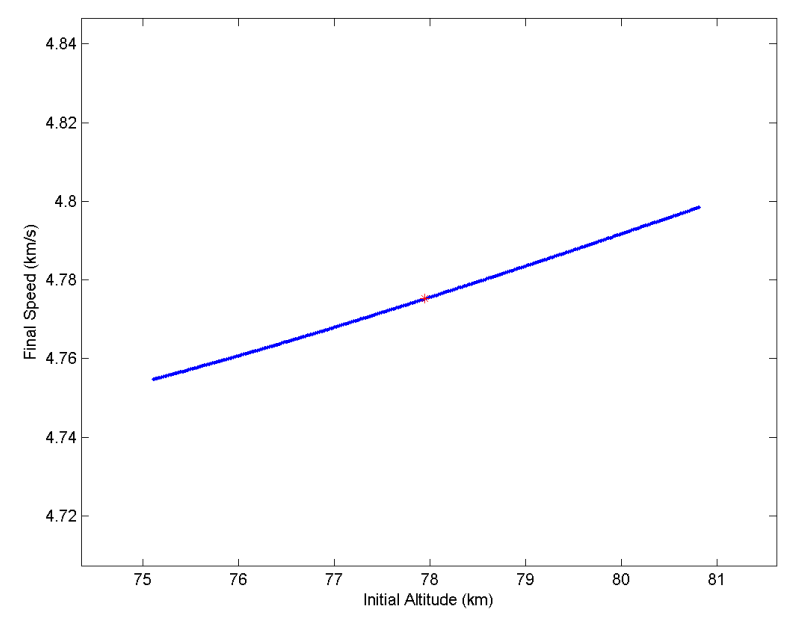

(b) Silver

Figure 5.21: Final Speed vs. Initial Altitude (Cylinder)

\subsubsection{Demise Altitude}

Demise altitude is the altitude at which the mass of the reentry object reaches zero. If the object does not reach zero mass, the impact altitude (identically zero) is instead plotted here. Molybdenum samples all survived to impact regardless of initial altitude in this simulation; all silver samples demised, with demise altitude depending weakly on initial altitude - a $7 \%$ increase in altitude corresponded to a $0.5 \%$ change in demise altitude.

\subsubsection{Sphere}

Molybdenum was chosen as one of the sample materials since its physical properties caused all its mass to survive until impact with the surface (seen as a horizontal line at zero in Figure 5.22a). The demise altitude for silver varied only by as much as $0.3 \%$ 
from the nominal demise altitude, and increased with increasing initial altitude (as seen in Figure 5.22b).

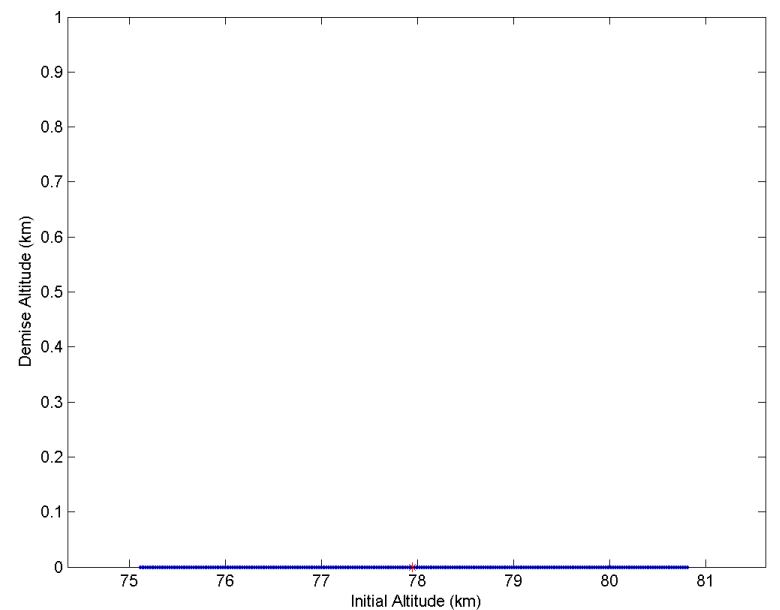

(a) Molybdenum

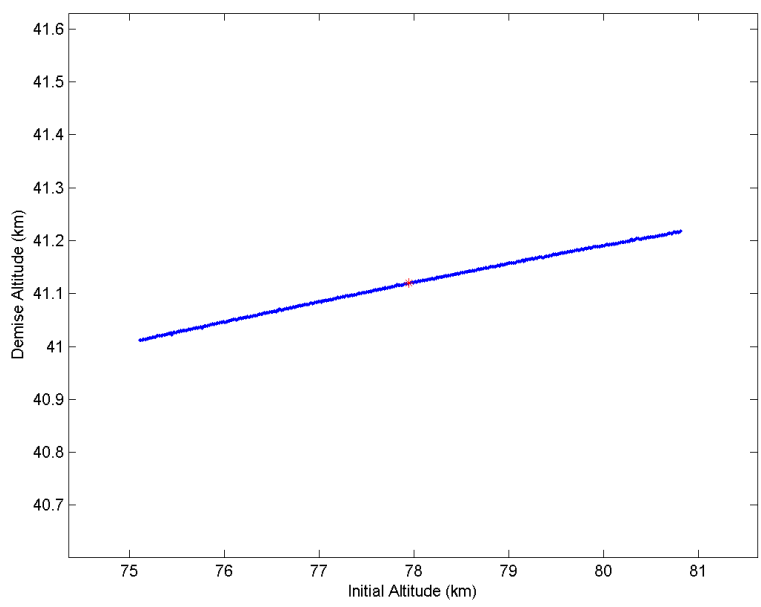

(b) Silver

\section{Figure 5.22: Demise Altitude vs. Initial Altitude (Sphere)}

\subsubsection{Cube}

Every molybdenum cube survives reentry to impact the surface, as seen in Figure 5.23a as a horizontal line at zero. Silver cubes have demise altitudes that vary by up to $0.5 \%$ of the nominal altitude, with the demise altitude increasing with initial altitude (see Figure $5.23 \mathrm{~b})$ 


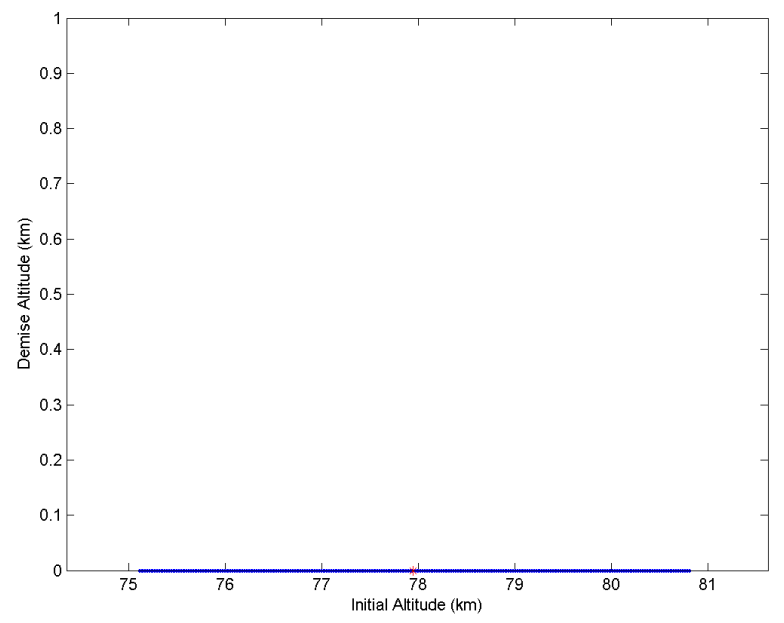

(a) Molybdenum

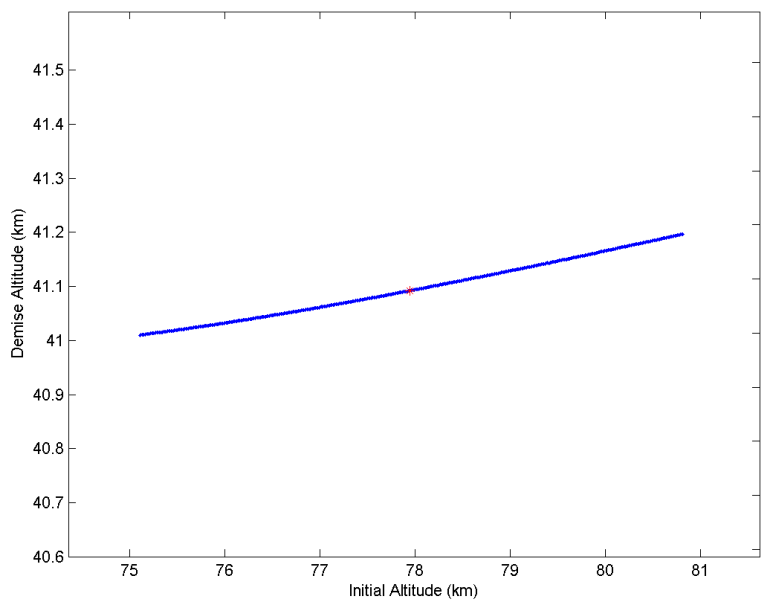

(b) Silver

Figure 5.23: Demise Altitude vs. Initial Altitude (Cube)

\subsubsection{Cylinder}

Cylinders had the same behavior as the cubes and spheres: molybdenum samples all survived reentry, and all silver samples demised, with the final altitude increasing with initial altitude (see Figures 5.24a and 5.24b, respectively).

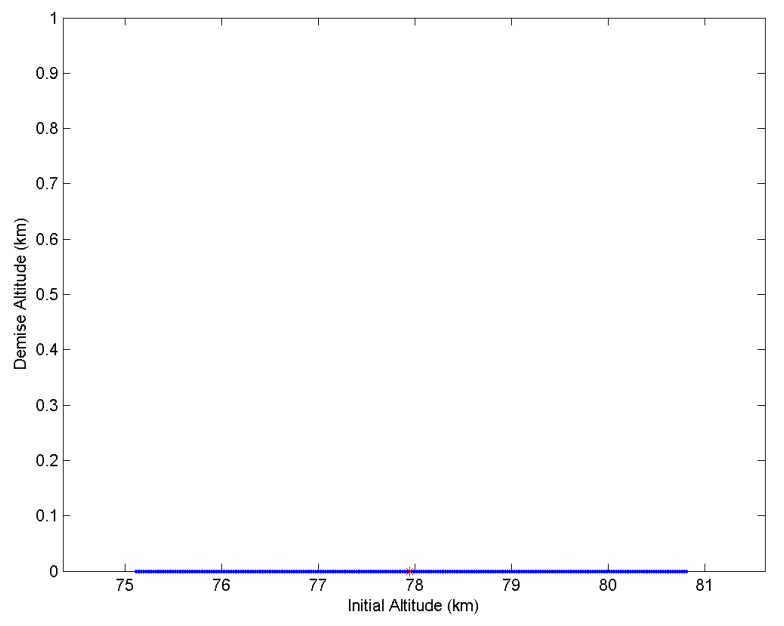

(a) Molybdenum

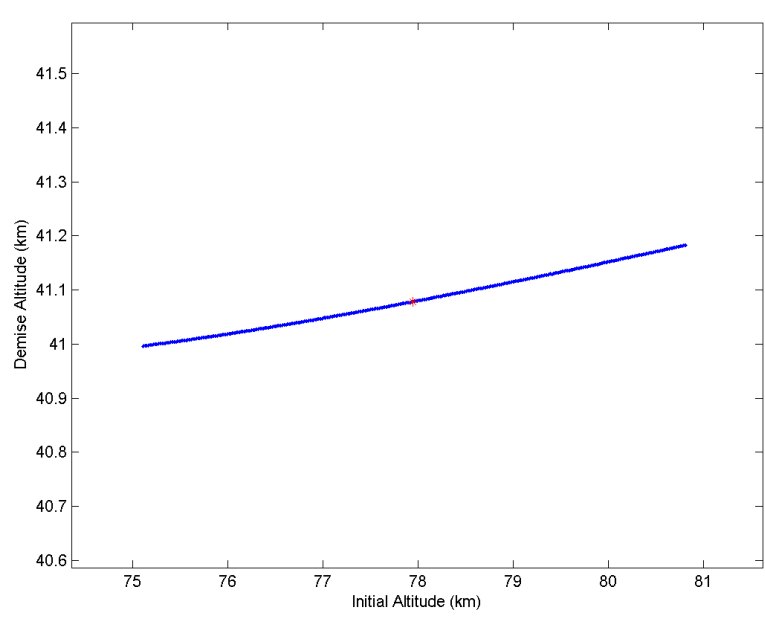

(b) Silver

Figure 5.24: Demise Altitude vs. Initial Altitude (Cylinder) 


\subsection{Freestream Temperature}

The third variable to be analyzed is the freestream temperature scaling factor, which will be varied from 0.75 to 1.25 (a constant multiplier applied throughout the simulation to the temperature returned from the atmos routine) over 500 simulated trajectories, and plotted against the final properties of interest as mentioned above: downrange distance, final mass, final altitude, and final impact speed.

\subsubsection{Downrange Distance}

Downrange distance is calculated as the length of the ground track from the initial position to either the demise of the body, or its impact with the surface. The effect of the freestream temperature scaling factor on downrange distance can be estimated, using Equation 5.3 as approximately 2 kilometers per percent change in temperature scaling.

$$
\frac{\partial D R}{\partial T_{\infty}} \approx \frac{\left.D R\right|_{T_{\infty, \max }}-\left.D R\right|_{T_{\infty, \min }}}{T_{\infty, \max }-T_{\infty, \min }}
$$

\subsubsection{Sphere}

The molybdenum and silver spheres both feature a trend toward shorter downrange distances to impact and demise, in Figures 5.25a and 5.25b respectively, as the temperature scaling factor increases. The increased freestream temperature increases drag and heating rates throughout the atmosphere. 


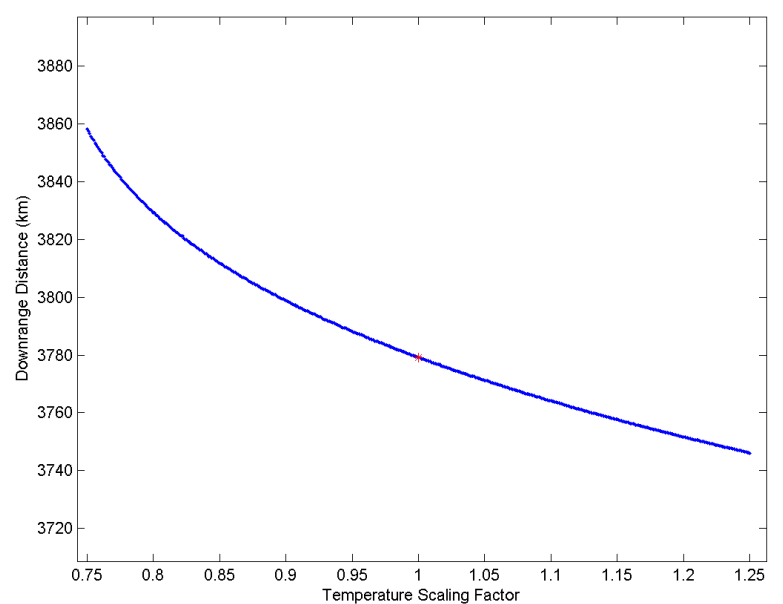

(a) Molybdenum

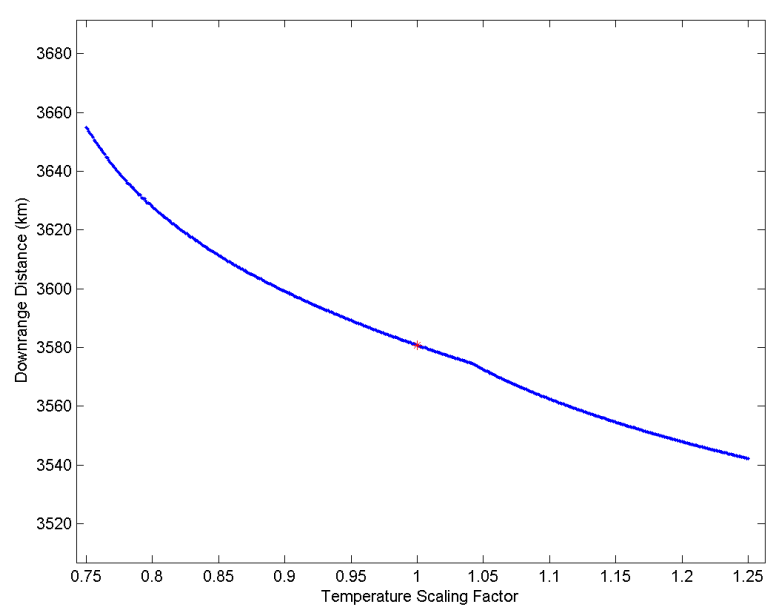

(b) Silver

Figure 5.25: Downrange Distance vs. Temperature Scaling Factor (Sphere)

\subsubsection{Cube}

Cubes tended to follow the same trend as the spheres in the section above, with the downrange distance decreasing with increasing freestream temperature scaling factor for both molybdenum and silver (see Figures 5.26a and 5.26b, respectively).

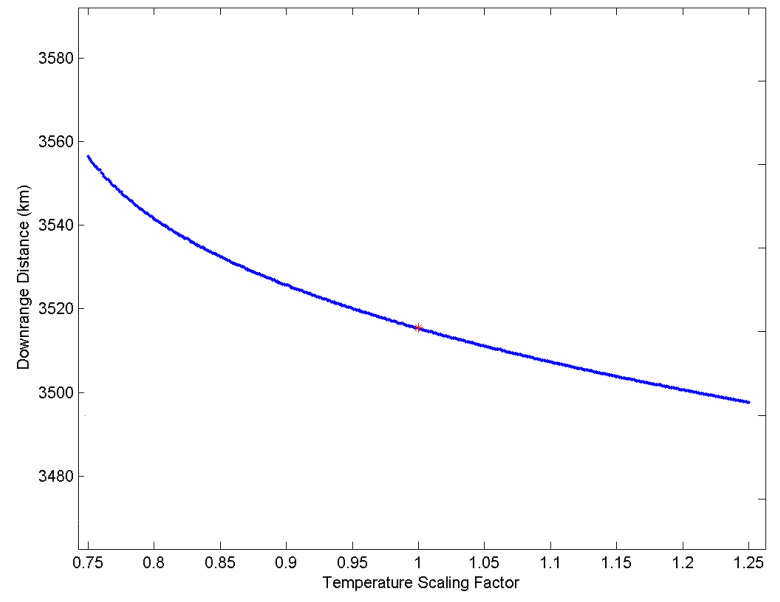

(a) Molybdenum

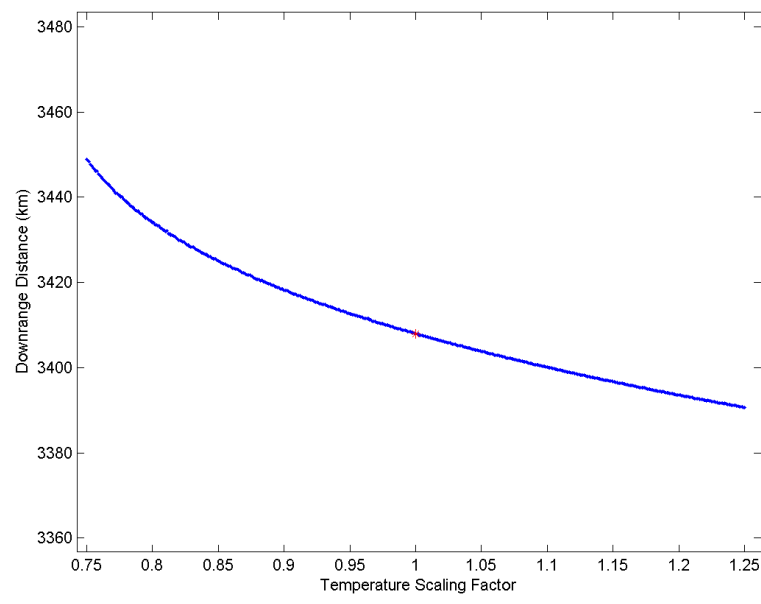

(b) Silver

Figure 5.26: Downrange Distance vs. Temperature Scaling Factor (Cube) 


\subsubsection{Cylinder}

Both materials in this analysis showed similar trends (see Figures 5.27a and 5.27b for molybdenum and silver, respectively) as the cubes and spheres, with increased freestream temperatures causing decreased final downrange distances.

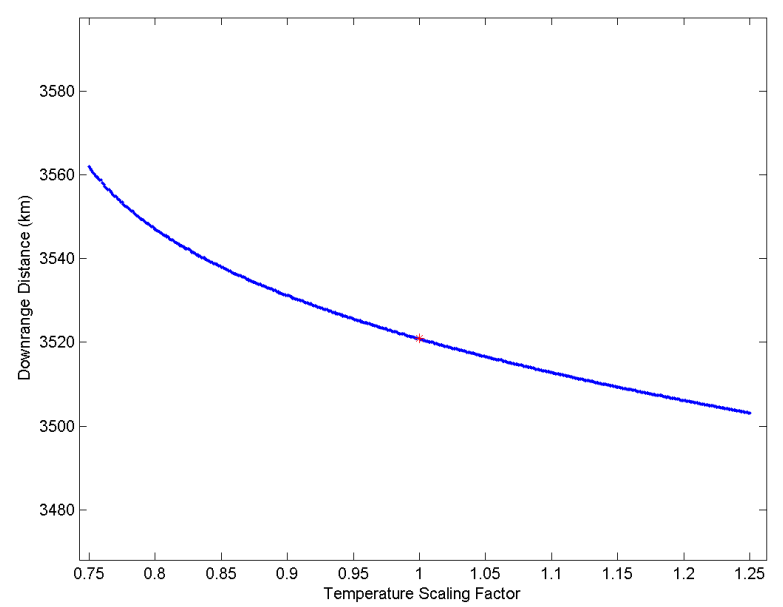

(a) Molybdenum

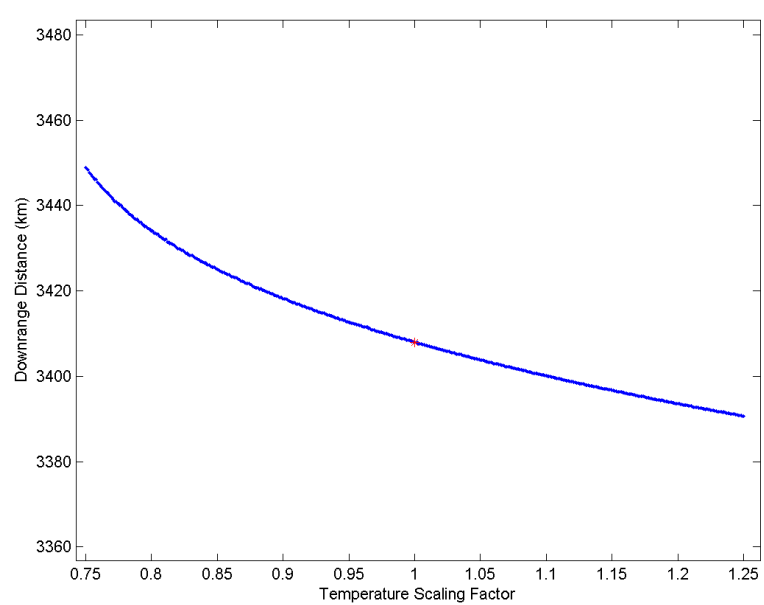

(b) Silver

Figure 5.27: Downrange Distance vs. Temperature Scaling Factor (Cylinder)

\subsubsection{Final Mass}

The final mass is, as expected, the mass at the end of each simulated trajectory: if the object demises, the mass is zero (and the following plot appears as a horizontal line at zero); if the object survives with no ablation, the mass is the same as its initial mass on-orbit (and the following plot would appear as a horizontal line at that value). Since no molybdenum sample lost mass during the simulated trajectories and every silver sample demised, it appears that (for these two classes of materials) the final mass is insensitive to changes in freestream temperature.

\subsubsection{Sphere}

Figures 5.28a and 5.28b appear similar (as horizontal lines), all the masses are identical for a material: the molybdenum samples did not lose any mass during the simulation, 
while all the silver samples demised before impact.

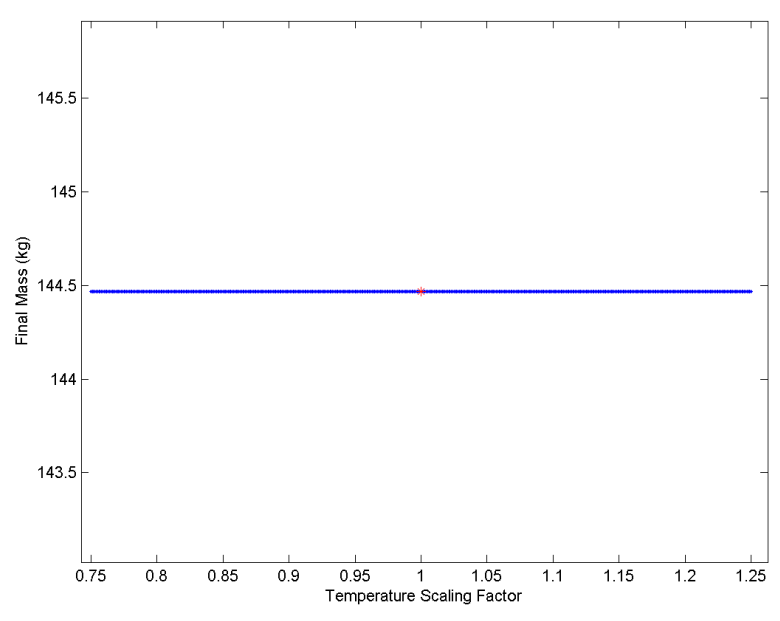

(a) Molybdenum

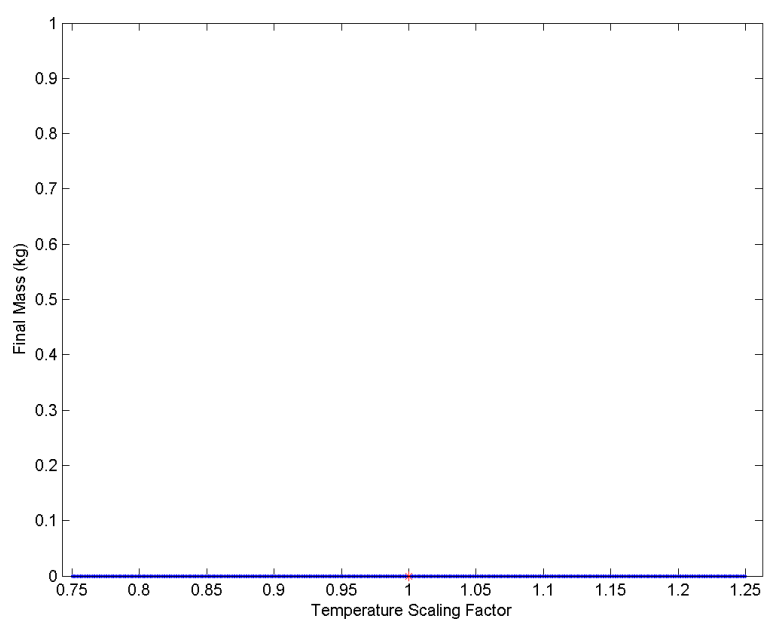

(b) Silver

Figure 5.28: Final Mass vs. Temperature Scaling Factor (Sphere)

\subsubsection{Cube}

As with the spheres, the cube masses did not vary with freestream temperature scaling for these materials: the molybdenum cubes lost no mass, and every silver cube demised before impact (seen as horizontal lines in Figures 5.29a and 5.29b, respectively).

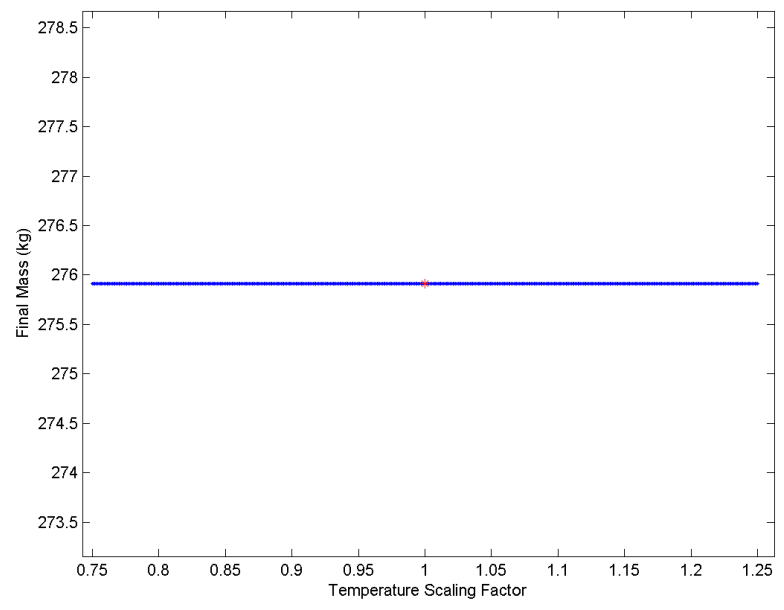

(a) Molybdenum

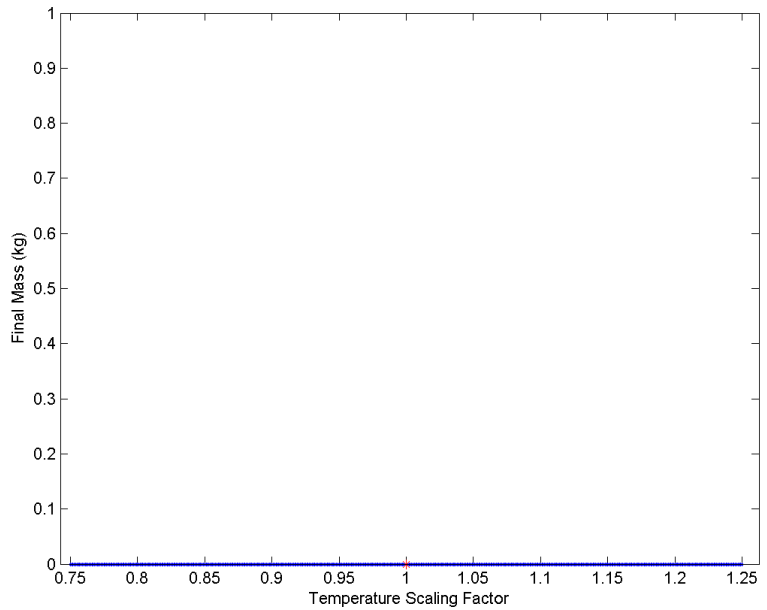

(b) Silver

Figure 5.29: Final Mass vs. Temperature Scaling Factor (Cube) 


\subsubsection{Cylinder}

Cylinder final masses are insensitive to the changes in freestream temperature simulated here, as indicated by the horizontal lines in Figures 5.30a and 5.30b, which show that the molybdenum samples remain at the initial mass and the silver samples all demise.

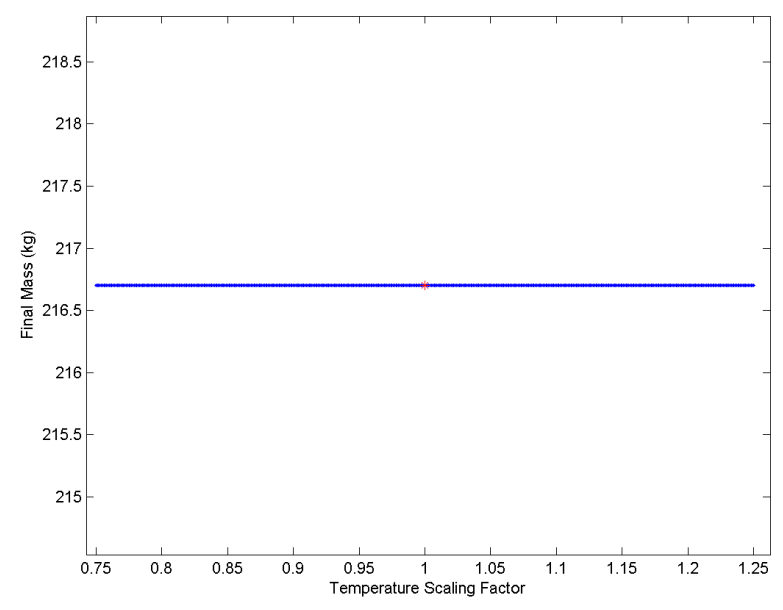

(a) Molybdenum

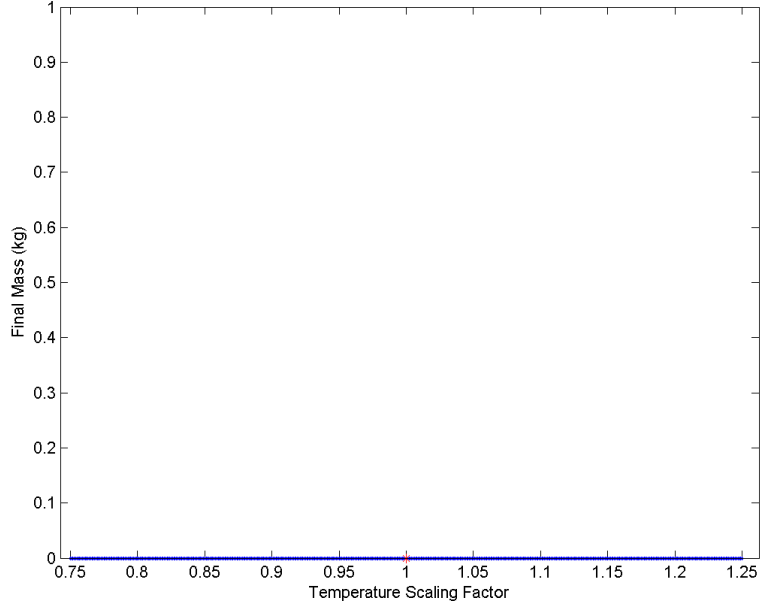

(b) Silver

Figure 5.30: Final Mass vs. Temperature Scaling Factor (Cylinder)

\subsubsection{Final Speed}

The final speed is the speed at either the demise of the reentry body, or at the body's impact with the Earth's surface. The impact speed of the molybdenum samples was insensitive to the freestream temperature. The final speed of the silver samples also varied little, only as much as $0.3 \%$ away from the nominal value.

\subsubsection{Sphere}

The trend in Figure 5.31a indicates that the effect of freestream temperature on atmospheric drag is minimal by the time the sample reaches the surface. In contrast, Figure $5.31 \mathrm{~b}$ shows that samples that demise can have slightly varying final speeds. The final speed of the silver spheres is related to the heating rate experienced in the upper atmosphere, which increases with the fourth power of temperature (as radiation); the drag 
increases linearly with temperature (related via density through Equation 1.2), having a lesser effect on the final speed.

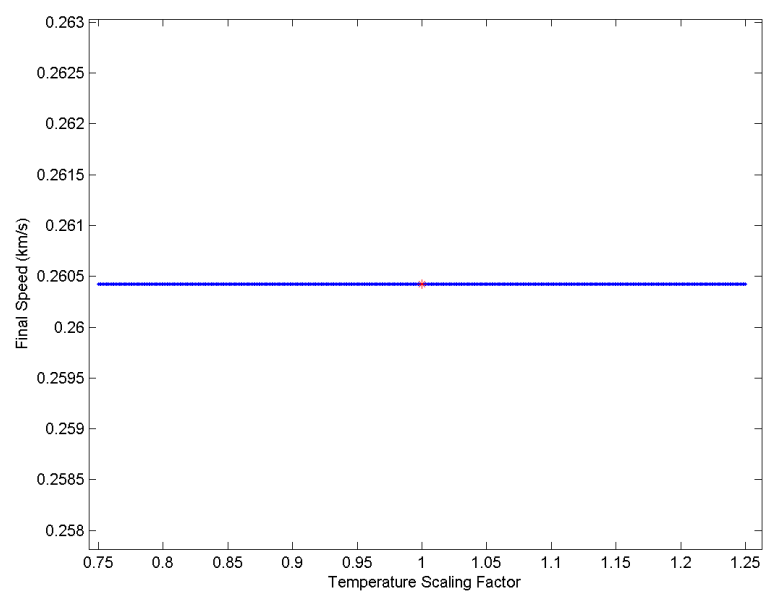

(a) Molybdenum

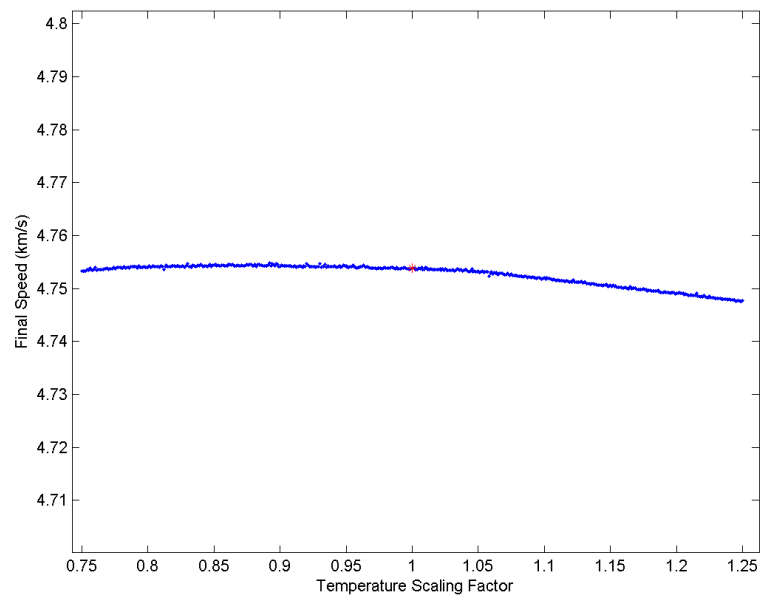

(b) Silver

Figure 5.31: Final Speed vs. Temperature Scaling Factor (Sphere)

\subsubsection{Cube}

As discussed above, the final speed of the molybdenum samples is invariant of freestream temperature (see horizontal line in Figure 5.32a). The silver cubes, as with the spheres, demonstrated a weak relationship between freestream temperature scaling and final speed (see Figure 5.32b). 


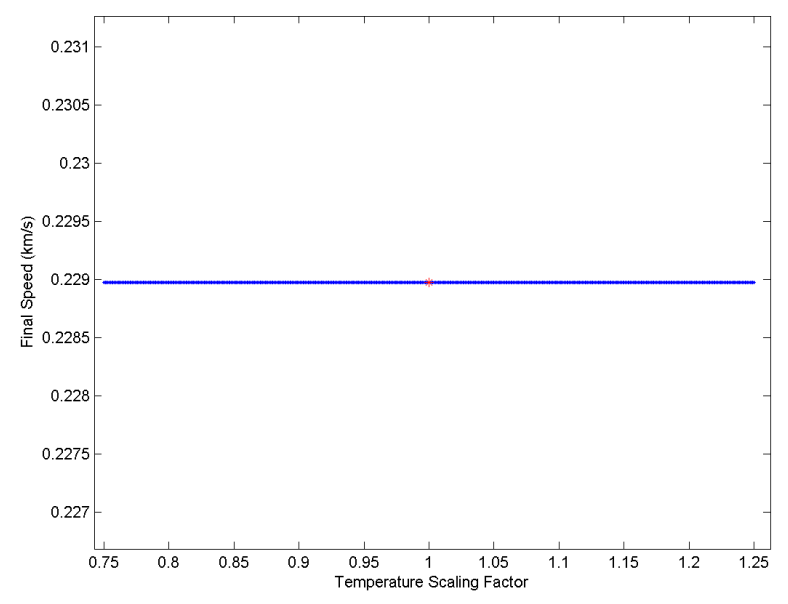

(a) Molybdenum

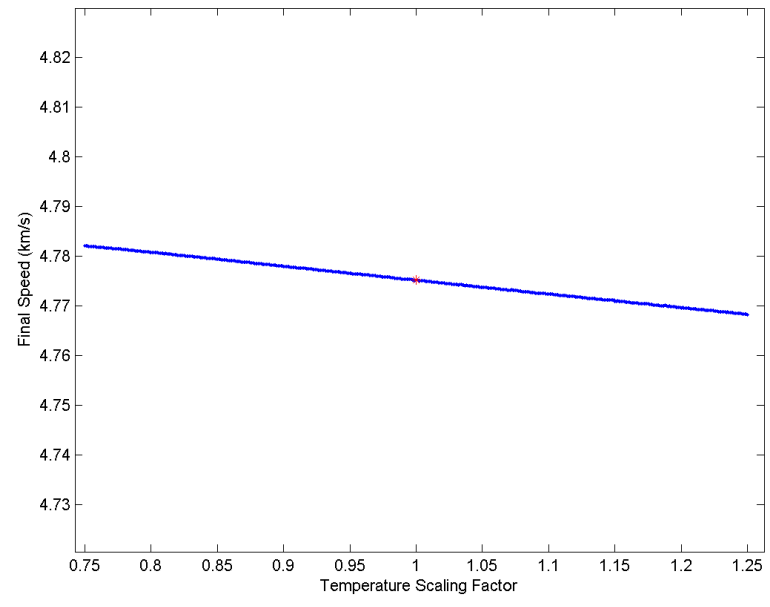

(b) Silver

Figure 5.32: Final Speed vs. Temperature Scaling Factor (Cube)

\subsubsection{Cylinder}

Again, the final speed of the molybdenum samples varies minimally with freestream temperature scaling (seen in Figure 5.33a); the effect of temperature on atmospheric drag is apparently minimized before impact. The final speed of the silver samples varies by up to $0.3 \%$ compared to the nominal temperature, as in Figure 5.33b.

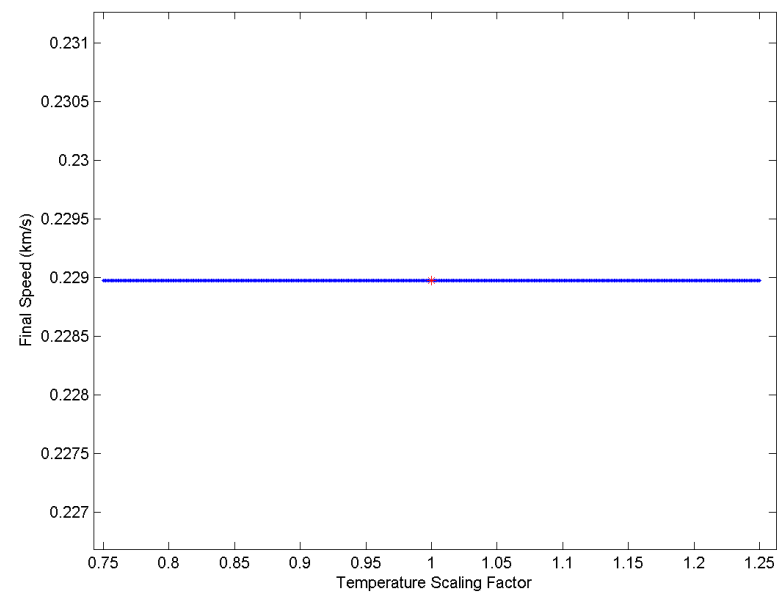

(a) Molybdenum

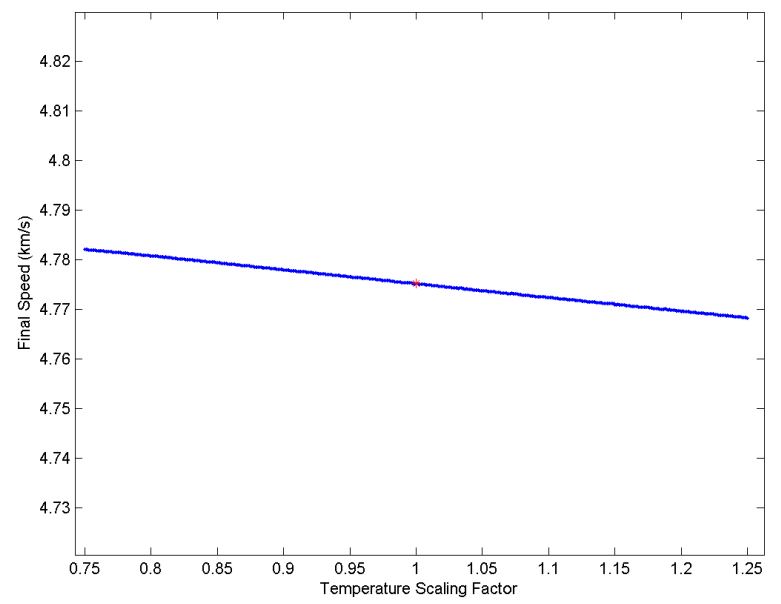

(b) Silver

Figure 5.33: Final Speed vs. Temperature Scaling Factor (Cylinder) 


\subsubsection{Demise Altitude}

Demise altitude is the altitude at which the mass of the reentry object reaches zero. If the object does not reach zero mass, the impact altitude (identically zero) is instead plotted here. Molybdenum samples all survived to impact regardless of freestream temperature scaling in this simulation; all silver samples demised, with demise altitude depending weakly on freestream temperature scaling - a $50 \%$ increase in temperature scale corresponded to less than a $0.1 \%$ change in demise altitude.

\subsubsection{Sphere}

Molybdenum was chosen as one of the sample materials since its physical properties caused all its mass to survive until impact with the surface (seen as a horizontal line at zero in Figure 5.34a). The demise altitude for silver varied by as much as $0.1 \%$ from the nominal demise altitude, and decreased very weakly with increasing temperature scaling factor (as seen in Figure 5.34b).

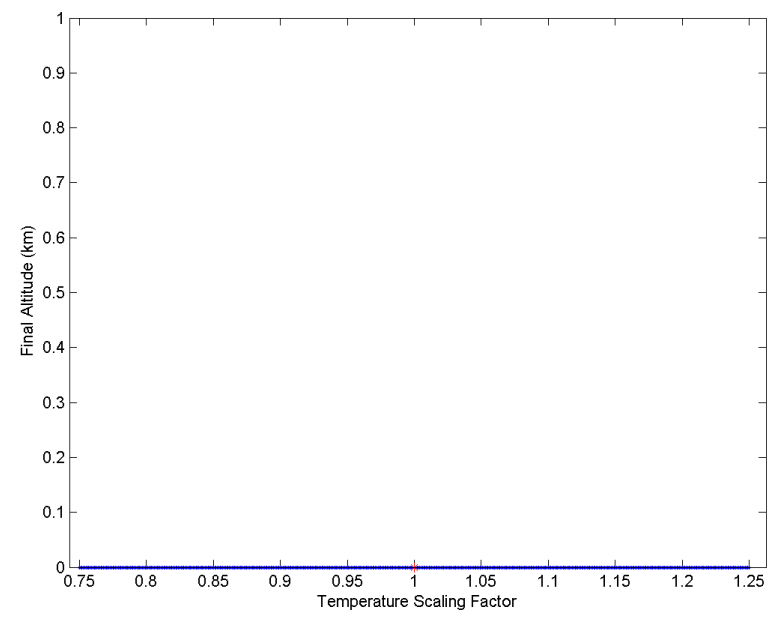

(a) Molybdenum

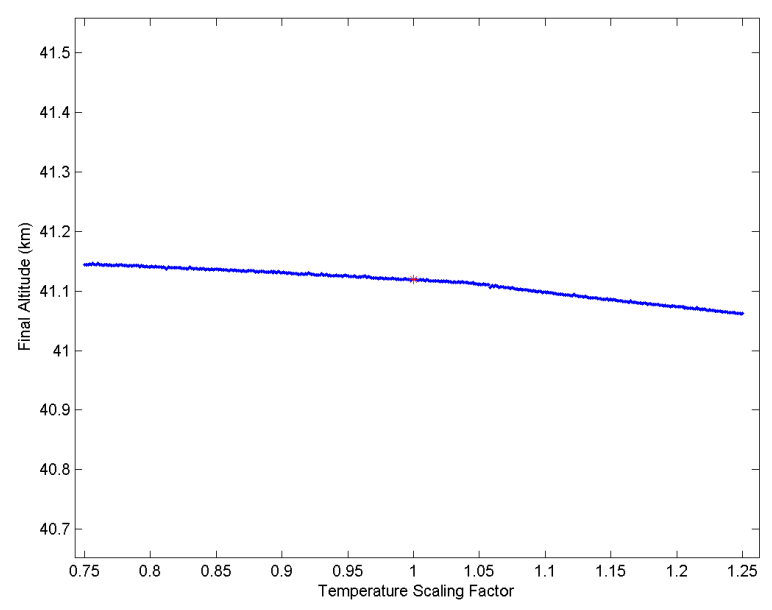

(b) Silver

Figure 5.34: Demise Altitude vs. Temperature Scaling Factor (Sphere) 


\subsubsection{Cube}

Every molybdenum cube survives reentry to impact the surface, as seen in Figure 5.35a as a horizontal line at zero. Silver cubes have demise altitudes that vary by up to $0.1 \%$ of the nominal altitude, with the demise altitude decreasing with freestream temperature scaling factor (see Figure 5.35b).

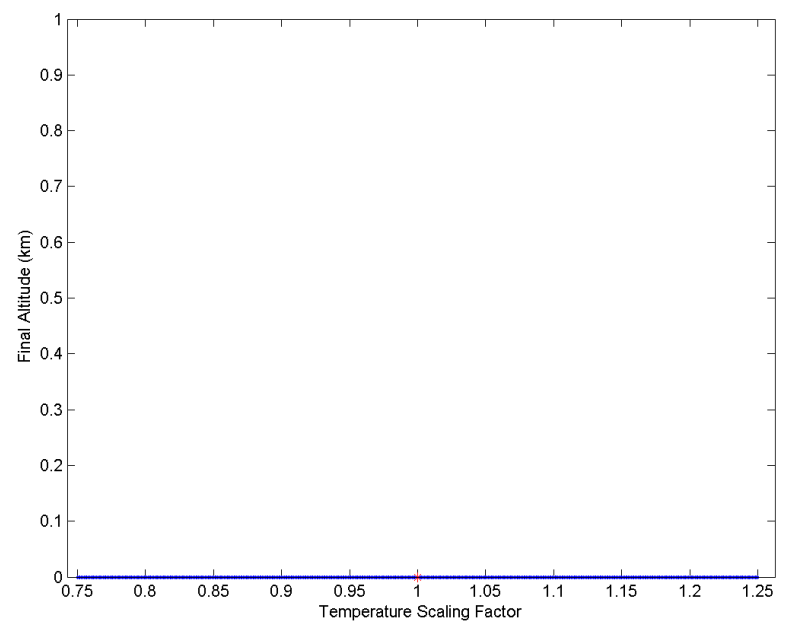

(a) Molybdenum

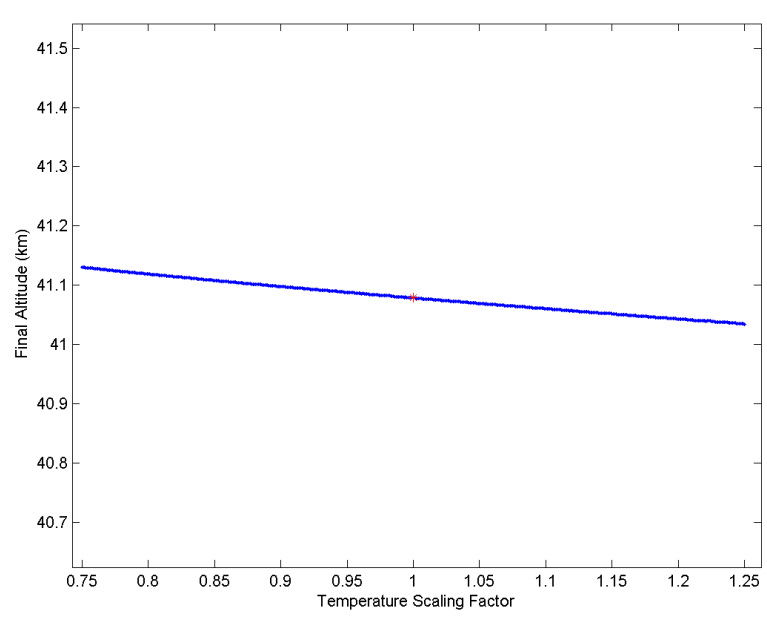

(b) Silver

Figure 5.35: Demise Altitude vs. Temperature Scaling Factor (Cube)

\subsubsection{Cylinder}

Cylinders had the same behavior as the cubes and spheres: molybdenum samples all survived reentry, and all silver samples demised, with the altitude slightly decreasing with freestream temperature scaling factor (see Figures 5.36a and 5.36b, respectively). 


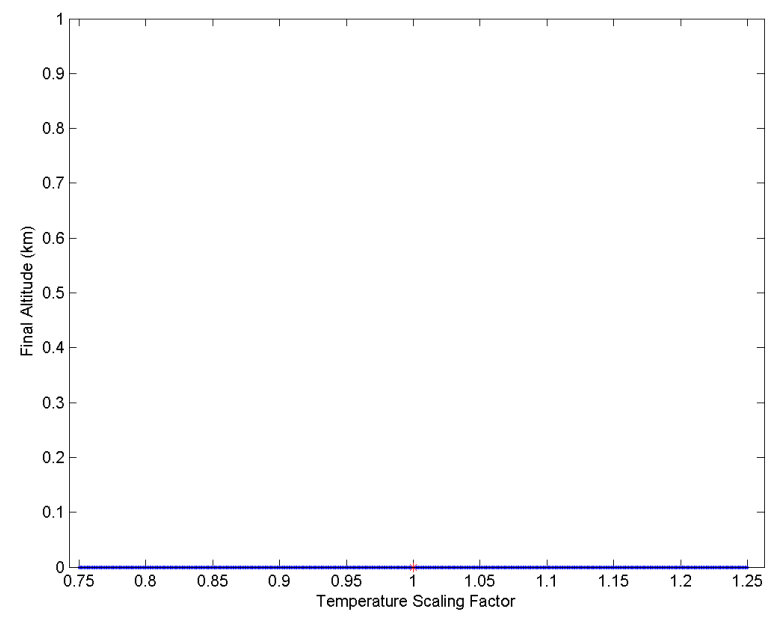

(a) Molybdenum

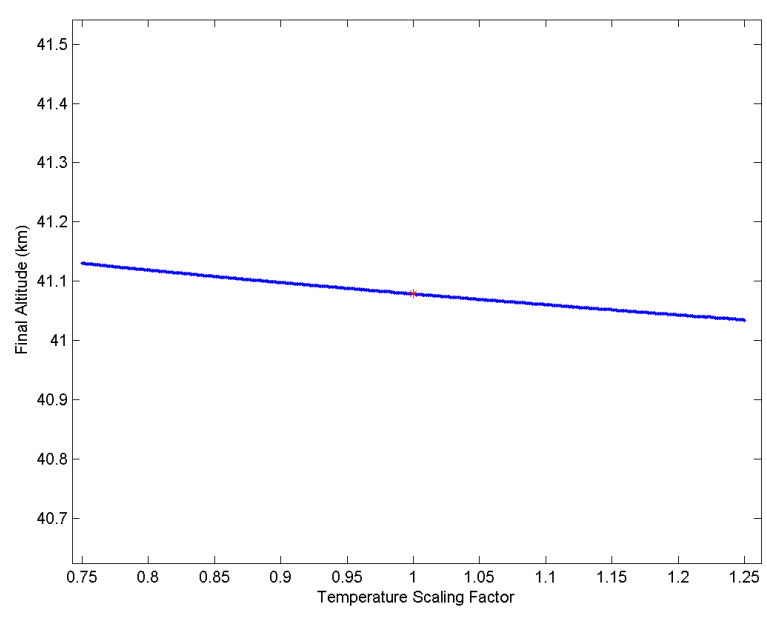

(b) Silver

Figure 5.36: Demise Altitude vs. Temperature Scaling Factor (Cylinder)

\subsection{Freestream Density}

The fourth variable to be analyzed is the freestream density scaling factor, which will be varied from 0.75 to 1.25 (a constant multiplier applied throughout the simulation to the density returned from the atmos routine) over 500 simulated trajectories, and plotted against the final properties of interest as mentioned above: downrange distance, final mass, final altitude, and final impact speed.

\subsubsection{Downrange Distance}

Downrange distance is calculated as the length of the ground track from the initial position to either the demise of the body, or its impact with the surface. The effect of the freestream temperature scaling factor on downrange distance can be estimated, using Equation 5.4 as between 0.5 and 3 kilometers per percent change in temperature scaling.

$$
\frac{\partial D R}{\partial \rho_{\infty}} \approx \frac{\left.D R\right|_{\rho_{\infty, \max }}-\left.D R\right|_{\rho_{\infty, \min }}}{\rho_{\infty, \max }-\rho_{\infty, \min }}
$$




\subsubsection{Sphere}

The molybdenum and silver spheres both feature a trend toward shorter downrange distances to impact and demise, in Figures 5.37a and 5.37b respectively, as the density scaling factor increases. The increased freestream density increases drag and heating rates throughout the atmosphere, causing the objects to descend and melt faster.

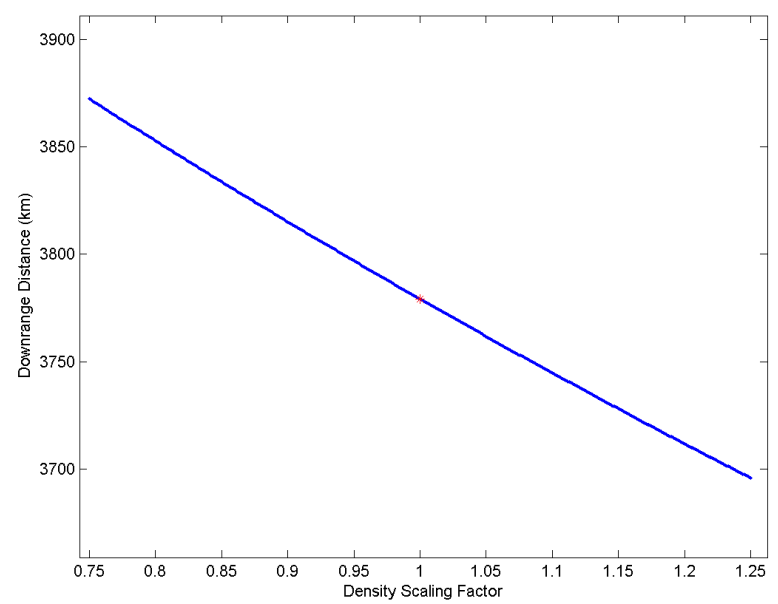

(a) Molybdenum

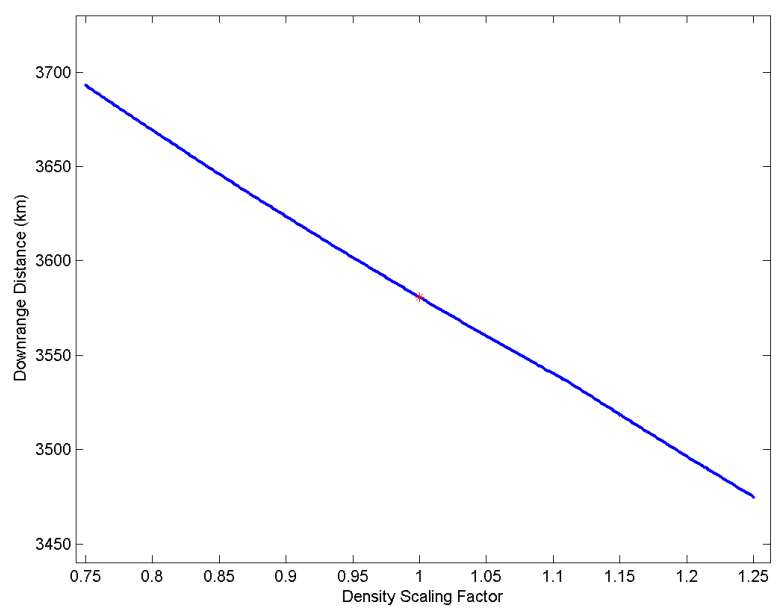

(b) Silver

\section{Figure 5.37: Downrange Distance vs. Density Scaling Factor (Sphere)}

\subsubsection{Cube}

Cubes tended to follow the same trend as the spheres in the section above, with the downrange distance decreasing with increasing freestream density scaling factor for both molybdenum and silver (see Figures 5.38a and 5.38b, respectively). 


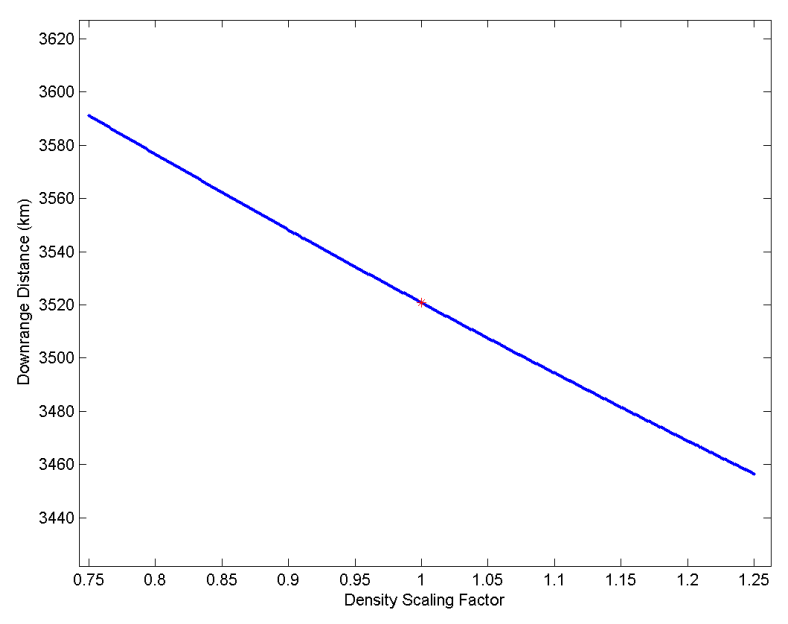

(a) Molybdenum

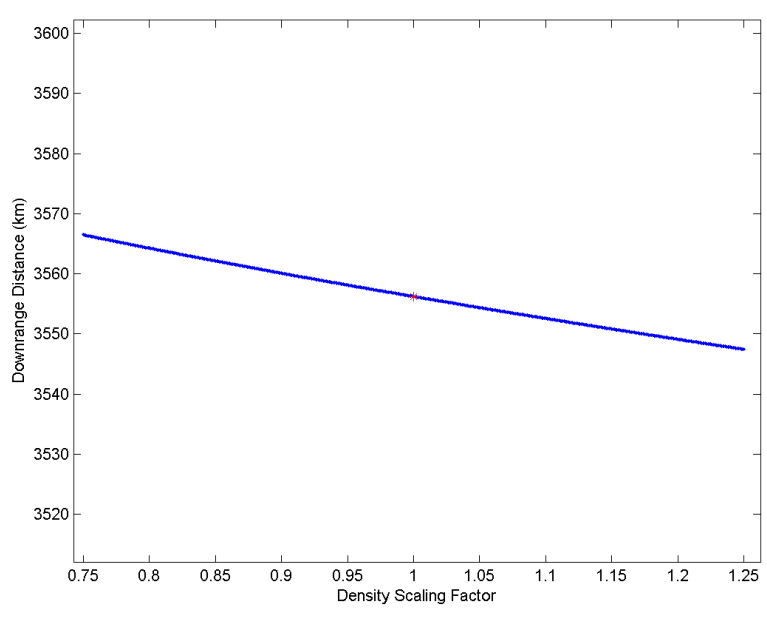

(b) Silver

Figure 5.38: Downrange Distance vs. Density Scaling Factor (Cube)

\subsubsection{Cylinder}

Both materials in this analysis showed similar trends (see Figures 5.39a and 5.39b for molybdenum and silver, respectively) as the cubes and spheres, with increased freestream densities causing decreased final downrange distances.

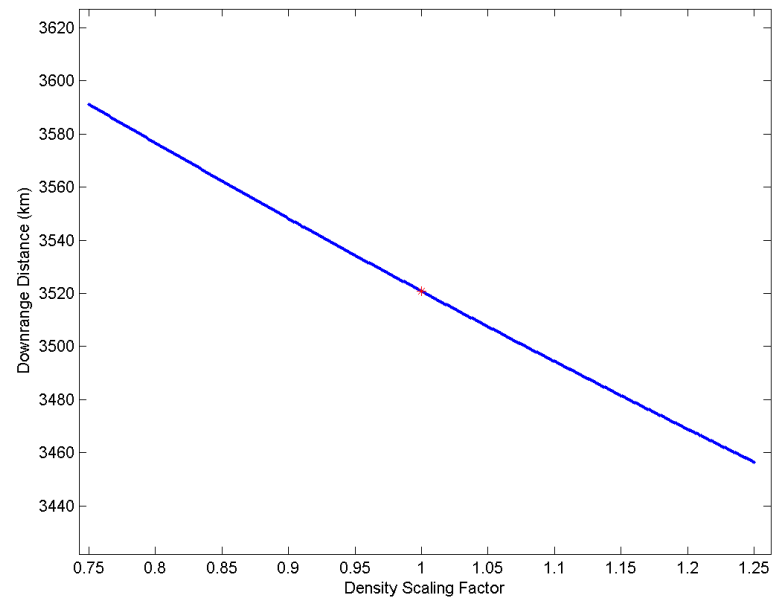

(a) Molybdenum

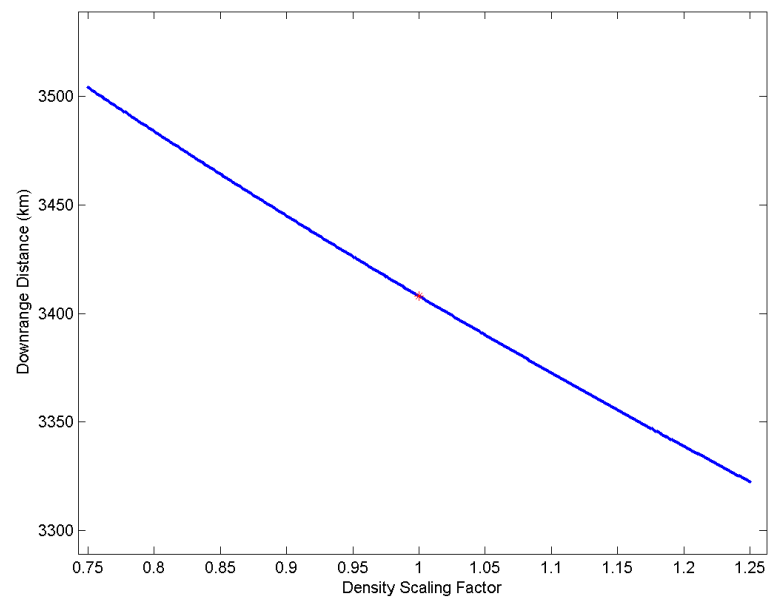

(b) Silver

Figure 5.39: Downrange Distance vs. Density Scaling Factor (Cylinder) 


\subsubsection{Final Mass}

The final mass is, as expected, the mass at the end of each simulated trajectory: if the object demises, the mass is zero (and the following plot appears as a horizontal line at zero); if the object survives with no ablation, the mass is the same as its initial mass on-orbit (and the following plot would appear as a horizontal line at that value). Since no molybdenum sample lost mass during the simulated trajectories and every silver sample demised, it appears that (for these two classes of materials) the final mass is insensitive to changes in freestream density.

\subsubsection{Sphere}

Figures 5.40a and 5.40b appear similar (as horizontal lines), all the masses are identical for a material: the molybdenum samples did not lose any mass during the simulation, while all the silver samples demised before impact.

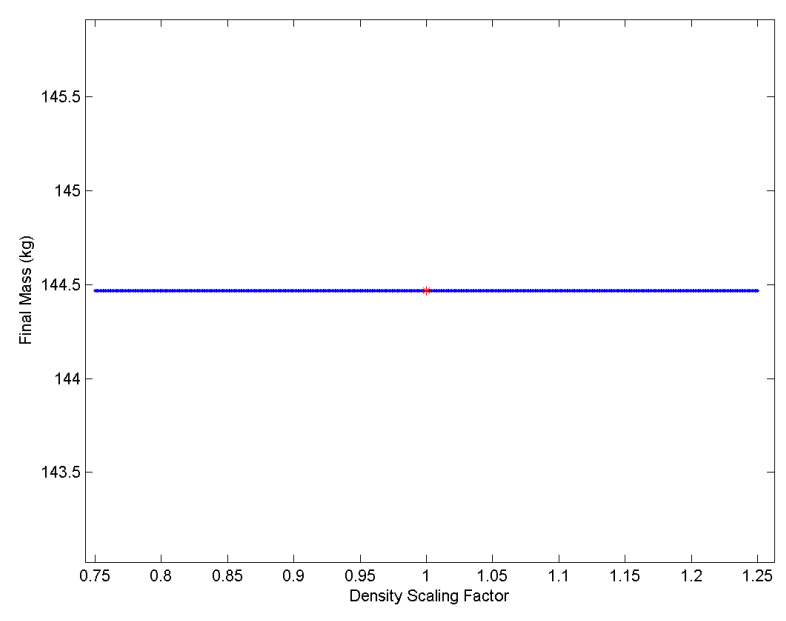

(a) Molybdenum

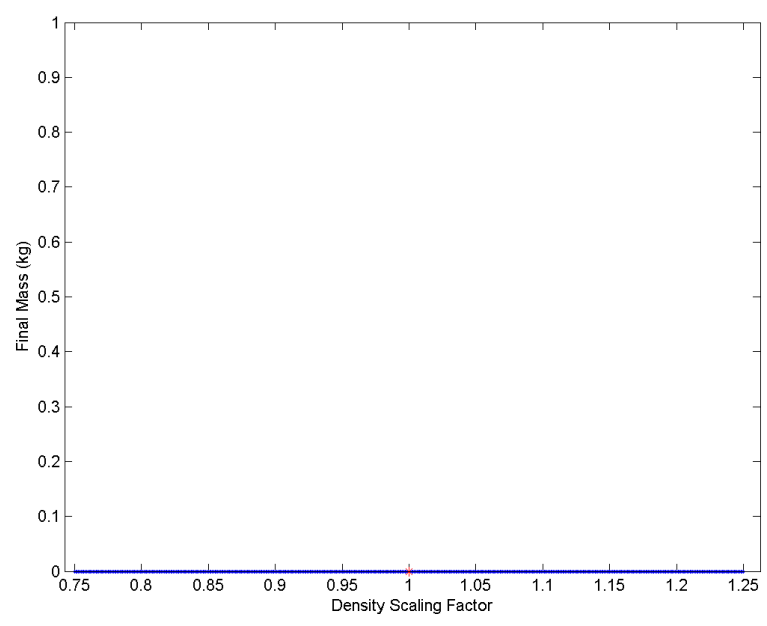

(b) Silver

Figure 5.40: Final Mass vs. Density Scaling Factor (Sphere)

\subsubsection{Cube}

As with the spheres, the cube masses did not vary with freestream density scaling for these materials: the molybdenum cubes lost no mass, and every silver cube demised 
before impact (seen as horizontal lines in Figures 5.41a and 5.41b, respectively).

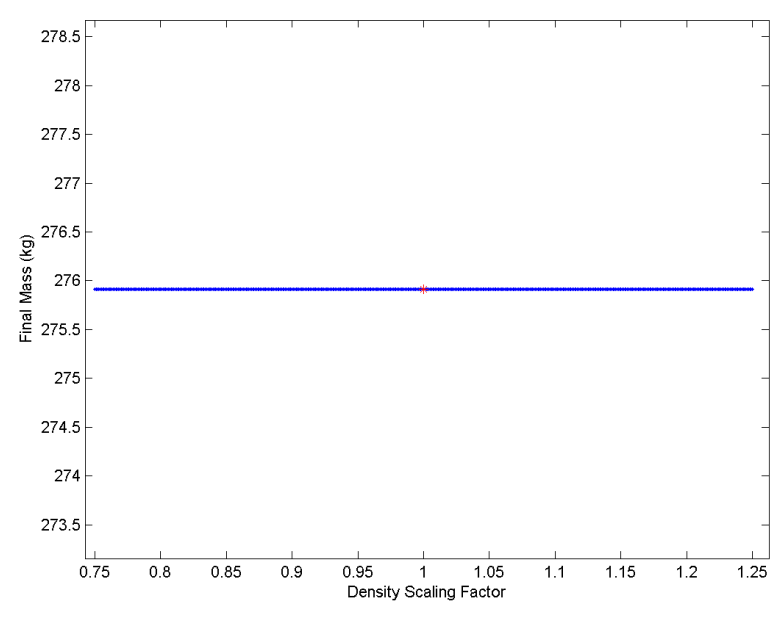

(a) Molybdenum

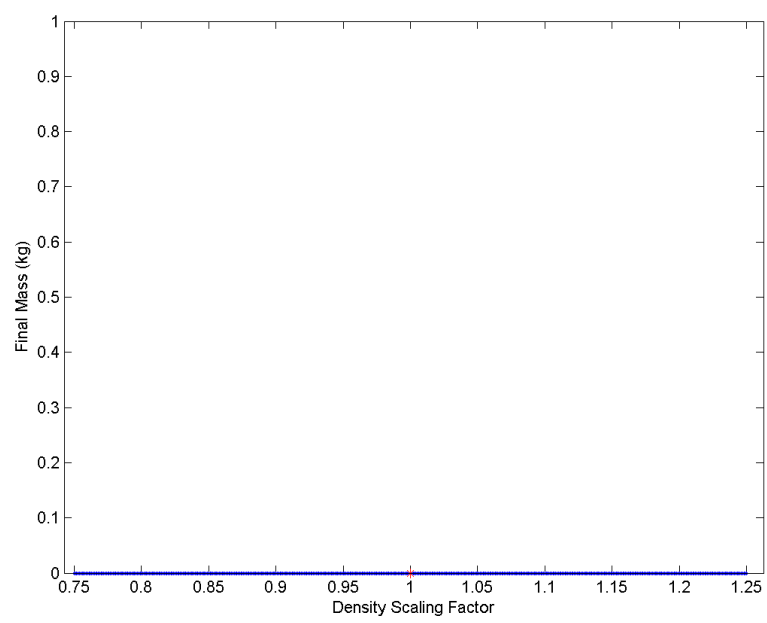

(b) Silver

Figure 5.41: Final Mass vs. Density Scaling Factor (Cube)

\subsubsection{Cylinder}

Cylinder final masses are insensitive to the changes in freestream density simulated here, as indicated by the horizontal lines in Figures $5.42 \mathrm{a}$ and $5.42 \mathrm{~b}$, which show that the molybdenum samples remain at the initial mass and the silver samples all demise.

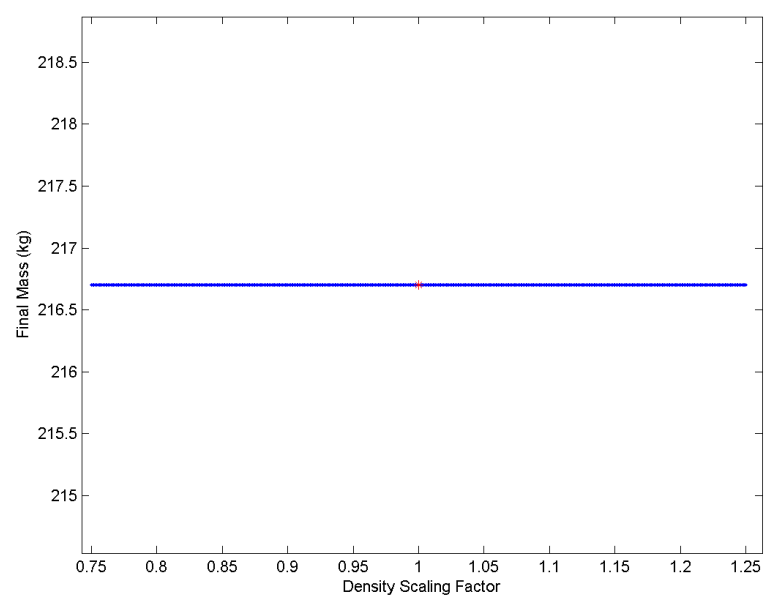

(a) Molybdenum

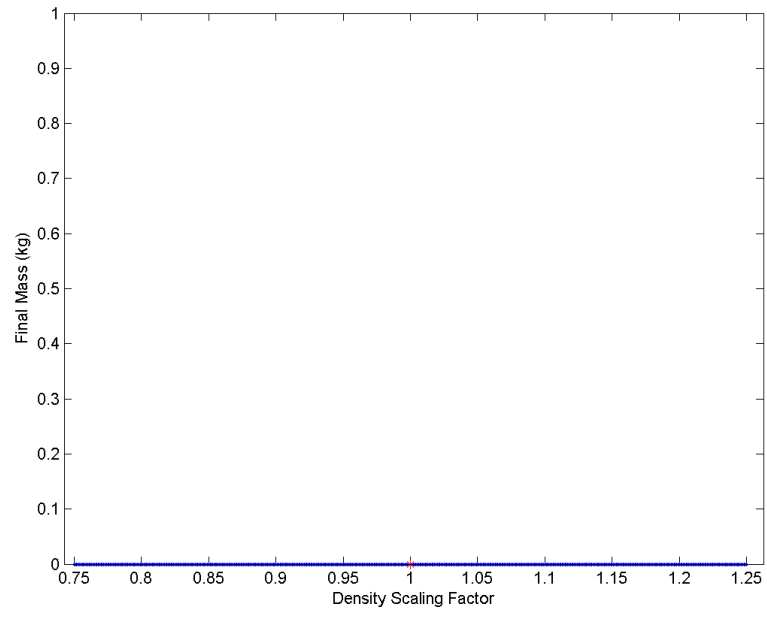

(b) Silver

Figure 5.42: Final Mass vs. Density Scaling Factor (Cylinder) 


\subsubsection{Final Speed}

The final speed is the speed at either the demise of the reentry body, or at the body's impact with the Earth's surface. The impact speed of the molybdenum samples was insensitive to the freestream density. The final speed of the silver samples also varied little, only as much as $1 \%$ away from the nominal value.

\subsubsection{Sphere}

The trend in Figure 5.43a indicates that the effect of freestream density scaling on atmospheric drag is minimal by the time the sample reaches the surface. In contrast, Figure 5.43b shows that samples that demise can have slightly varying final speeds. The final speed of the silver spheres is related to the heating rate experienced in the upper atmosphere, which increases linearly with density (as in Equation 3.35); the drag also increases linearly with density, but has a lesser effect on the final speed.

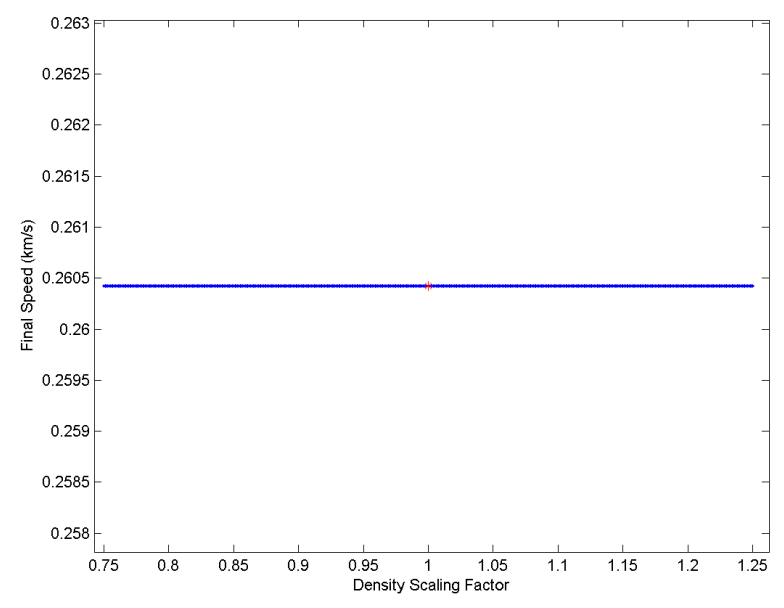

(a) Molybdenum

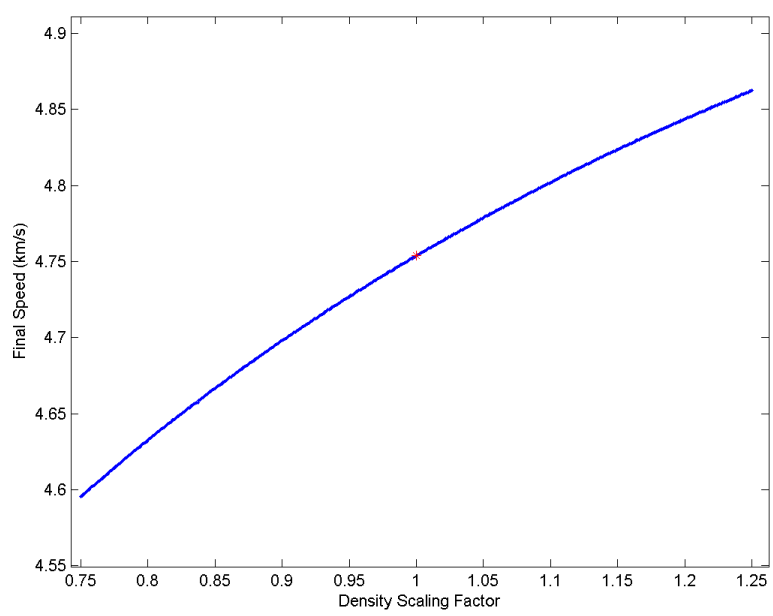

(b) Silver

Figure 5.43: Final Speed vs. Density Scaling Factor (Sphere)

\subsubsection{Cube}

As discussed above, the final speed of the molybdenum samples is invariant of freestream density (see horizontal line in Figure 5.44a). The silver cubes, as with the spheres, 
demonstrated a weak relationship between freestream density scaling and final speed (see Figure 5.44b).

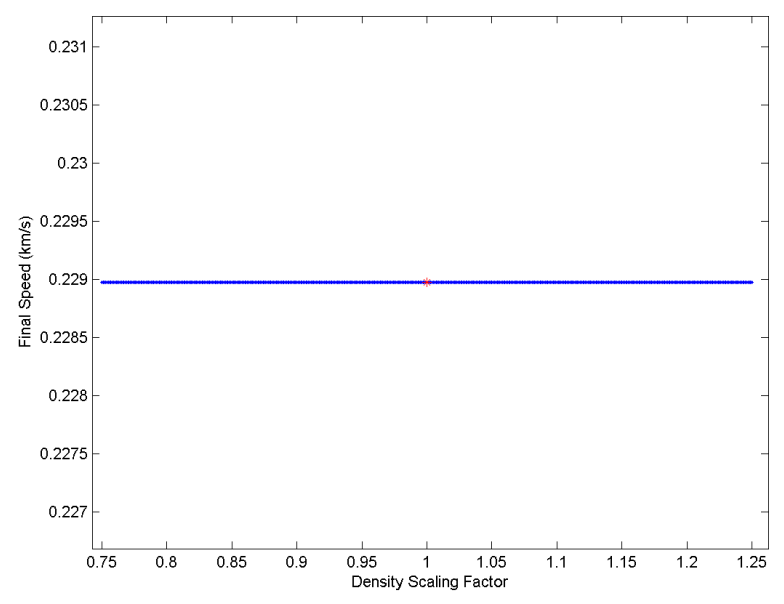

(a) Molybdenum

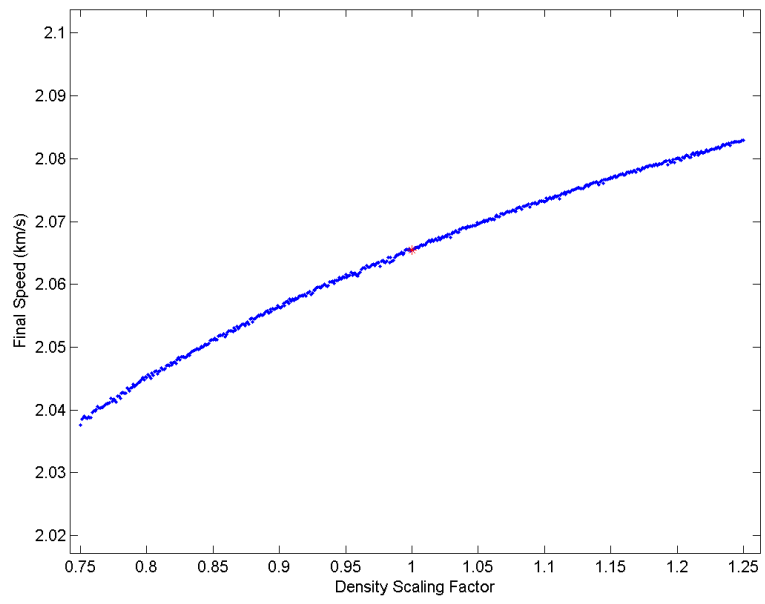

(b) Silver

Figure 5.44: Final Speed vs. Density Scaling Factor (Cube)

\subsubsection{Cylinder}

Again, the final speed of the molybdenum samples varies minimally with freestream density scaling (seen in Figure 5.45a); the effect of density on atmospheric drag is apparently minimized before impact. The final speed of the silver samples varies by up to $0.3 \%$ compared to the nominal density, as in Figure 5.45b. 


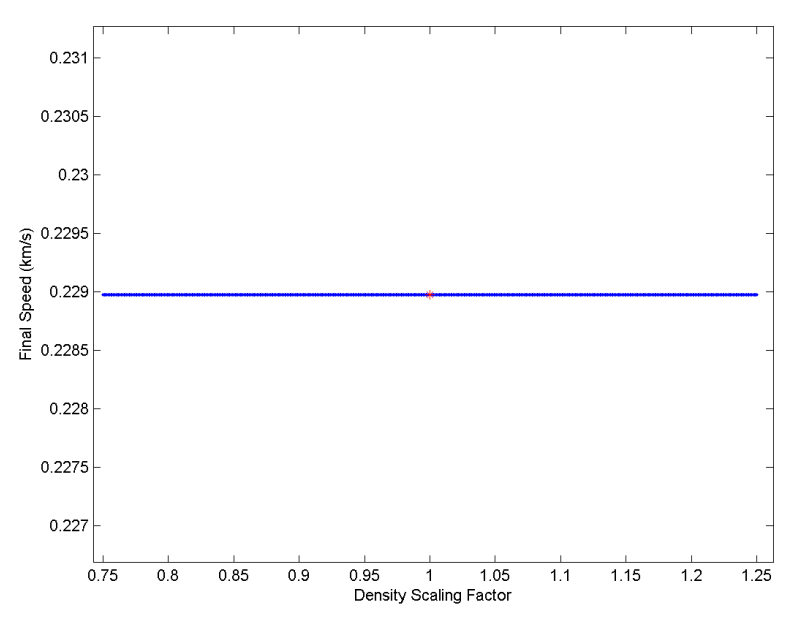

(a) Molybdenum

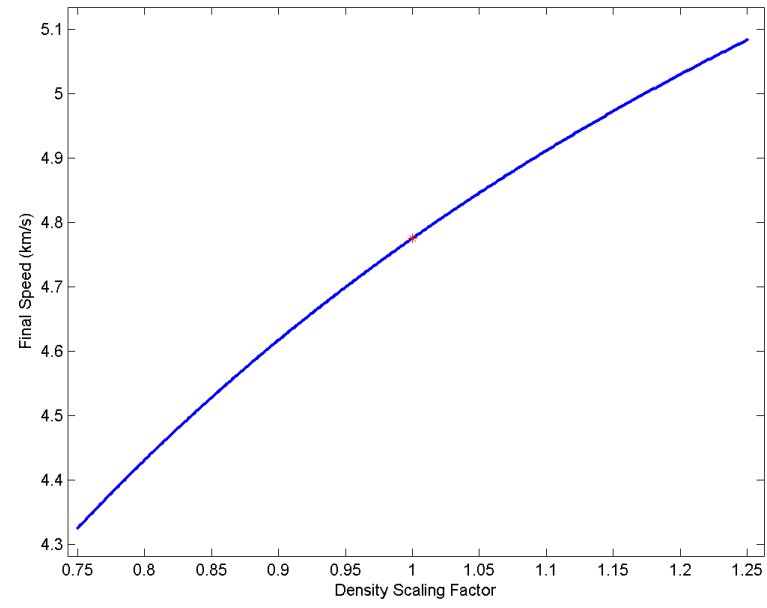

(b) Silver

Figure 5.45: Final Speed vs. Density Scaling Factor (Cylinder)

\subsubsection{Demise Altitude}

Demise altitude is the altitude at which the mass of the reentry object reaches zero. If the object does not reach zero mass, the impact altitude (identically zero) is instead plotted here. Molybdenum samples all survived to impact regardless of freestream density scaling in this simulation; all silver samples demised, with demise altitude depending on freestream density scaling - a $50 \%$ increase in density scale corresponded to an almost $10 \%$ change in demise altitude.

\subsubsection{Sphere}

Molybdenum was chosen as one of the sample materials since its physical properties caused all its mass to survive until impact with the surface (seen as a horizontal line at zero in Figure 5.46a). The demise altitude for silver varied by as much as $1 \%$ from the nominal demise altitude, and increased very weakly with increasing density scaling factor (as seen in Figure 5.46b). 


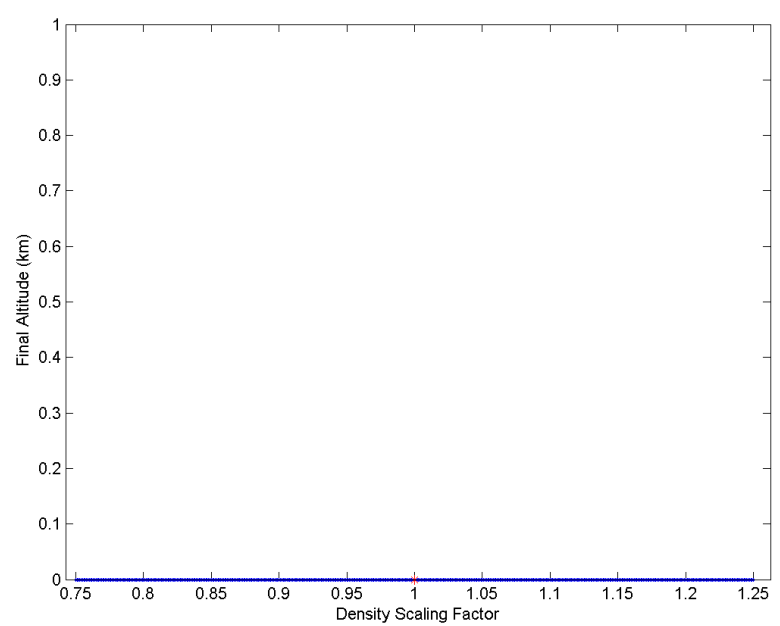

(a) Molybdenum

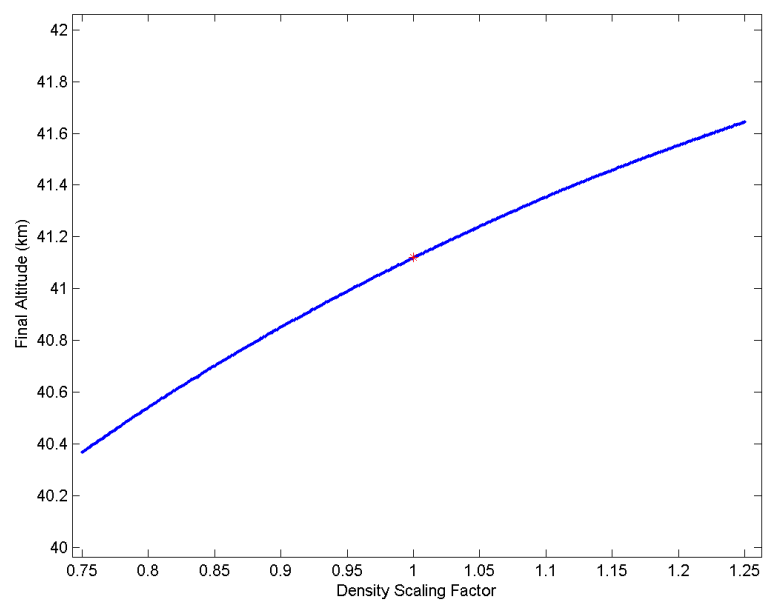

(b) Silver

Figure 5.46: Demise Altitude vs. Density Scaling Factor (Sphere)

\subsubsection{Cube}

Every molybdenum cube survives reentry to impact the surface, as seen in Figure 5.47a as a horizontal line at zero. Silver cubes have demise altitudes that vary by up to $1 \%$ of the nominal altitude, with the demise altitude decreasing with freestream density scaling factor (see Figure 5.47b).

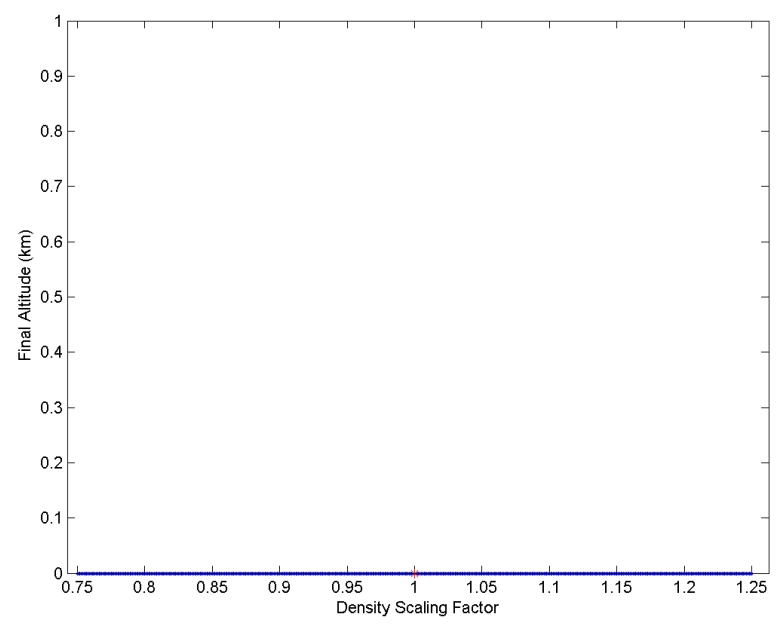

(a) Molybdenum

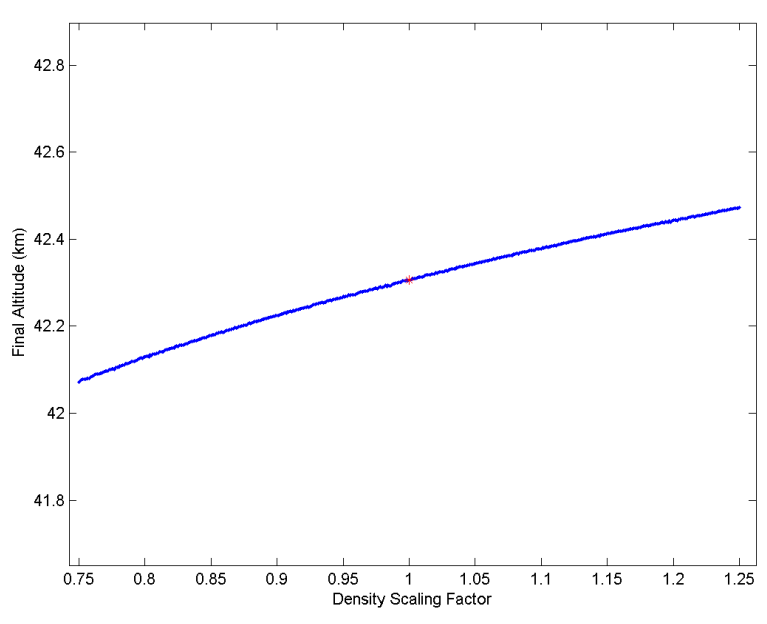

(b) Silver

Figure 5.47: Demise Altitude vs. Density Scaling Factor (Cube) 


\subsubsection{Cylinder}

Cylinders had the same behavior as the cubes and spheres: molybdenum samples all survived reentry, and all silver samples demised, with the altitude increasing by almost $5 \%$ with freestream density scaling factor (see Figures 5.48a and 5.48b, respectively).

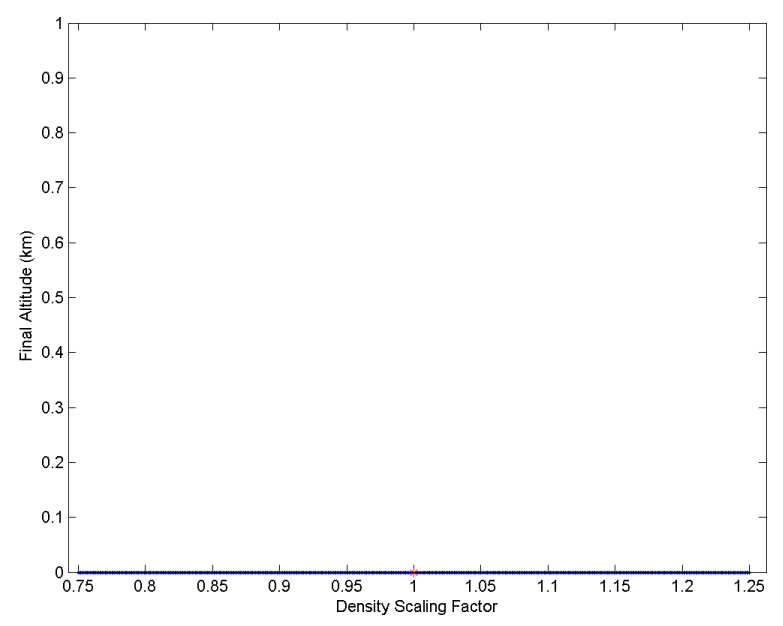

(a) Molybdenum

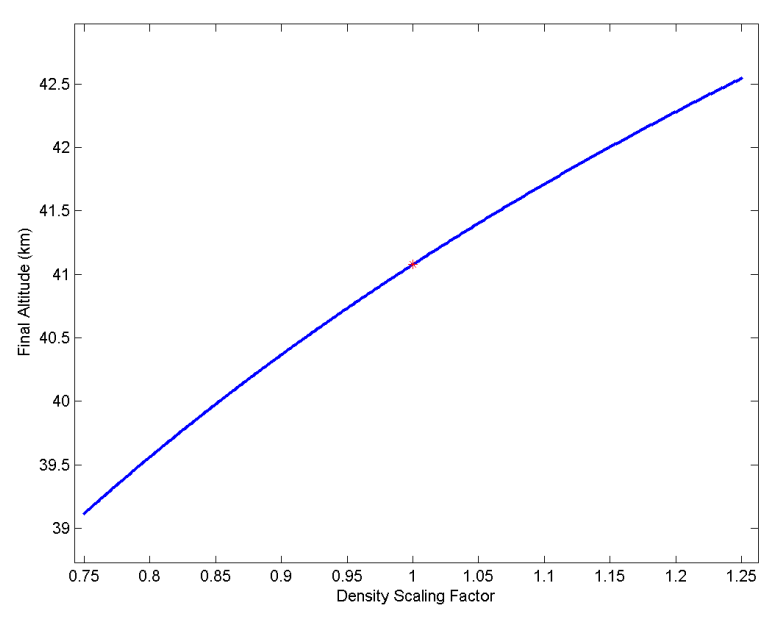

(b) Silver

Figure 5.48: Demise Altitude vs. Density Scaling Factor (Cylinder)

\subsection{Initial Temperature}

The final variable to be analyzed is the initial body temperature, which will be varied from 150 to $600 \mathrm{~K}$ over 500 simulated trajectories, and plotted against the final properties of interest as mentioned above: downrange distance, final mass, final altitude, and final impact speed.

\subsubsection{Downrange Distance}

Downrange distance is calculated as the length of the ground track from the initial position to either the demise of the body, or its impact with the surface. The effect of the initial body temperature on downrange distance can be estimated, using Equation 
5.5 as approximately 2 kilometers per Kelvin.

$$
\frac{\partial D R}{\partial T_{\text {body }}} \approx \frac{\left.D R\right|_{T_{\text {body,max }}}-\left.D R\right|_{T_{\text {body,min }}}}{T_{\text {body,max }}-T_{\text {body, min }}}
$$

\subsubsection{Sphere}

Since the thermal energy accepted by the molybdenum spheres is nowhere near enough to melt them, even with the initial temperature at $600 \mathrm{~K}$, the downrange distance is insensitive to initial temperature (seen as a horizontal line in 5.49a). Since silver spheres accept enough thermal energy to completely melt from an initial temperature of 300K, any higher initial temperature will simply cause the ablation to occur sooner; lower initial temperatures will cause the process to take longer (see Figure 5.49b).

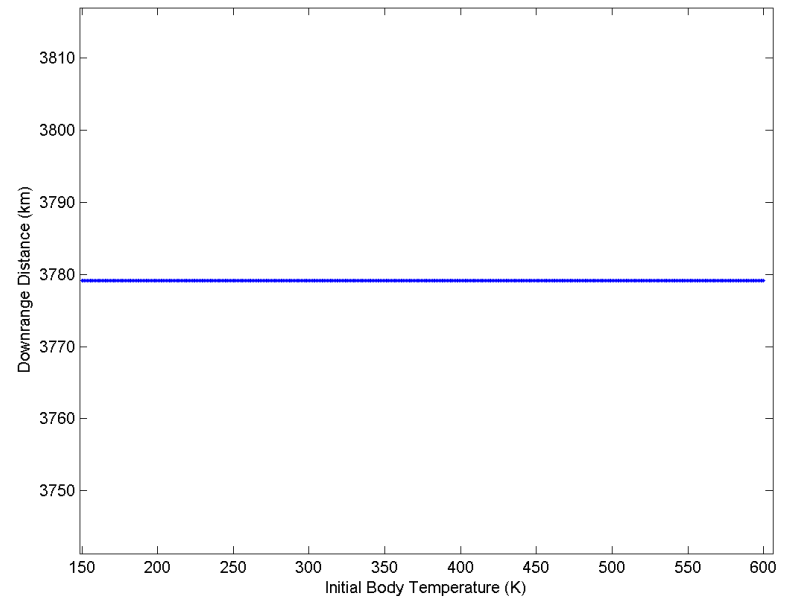

(a) Molybdenum

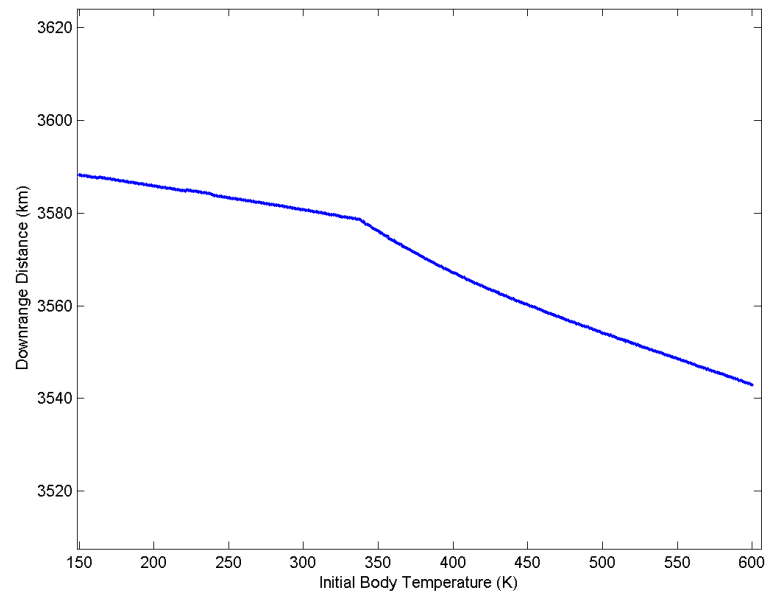

(b) Silver

Figure 5.49: Downrange Distance vs. Initial Temperature (Sphere)

\subsubsection{Cube}

Cubes tended to follow the same trend as the spheres in the section above, with the downrange distance remaining constant for molybdenum and decreasing with increasing initial temperature for silver (see Figures 5.50a and 5.50b, respectively). 


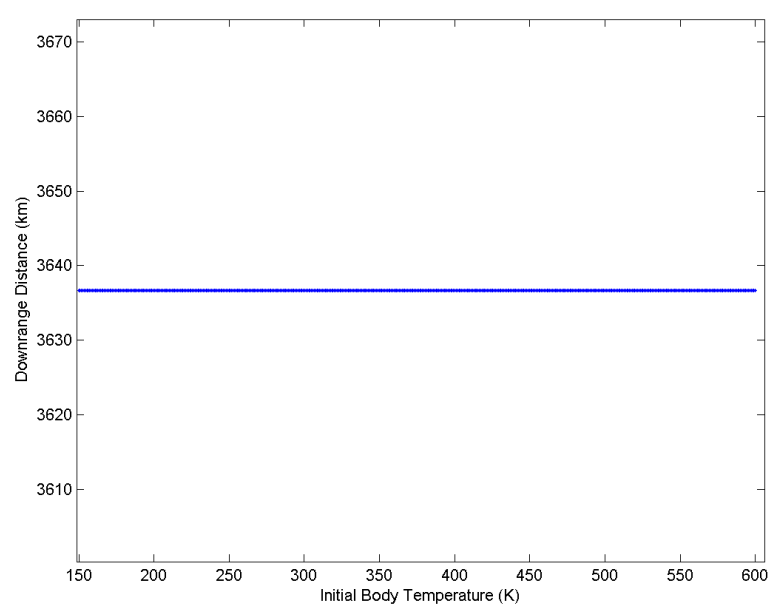

(a) Molybdenum

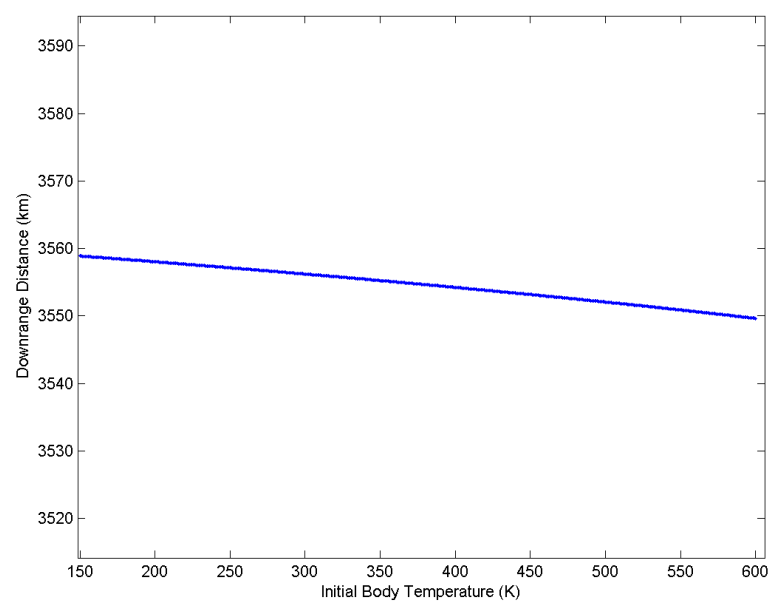

(b) Silver

Figure 5.50: Downrange Distance vs. Initial Temperature (Cube)

\subsubsection{Cylinder}

Both materials in this analysis showed similar trends as with the spheres and cubes (see Figures 5.51a and 5.51b for molybdenum and silver, respectively), with increased initial temperatures causing decreased final downrange distances for silver samples, and no effect on molybdenum samples.

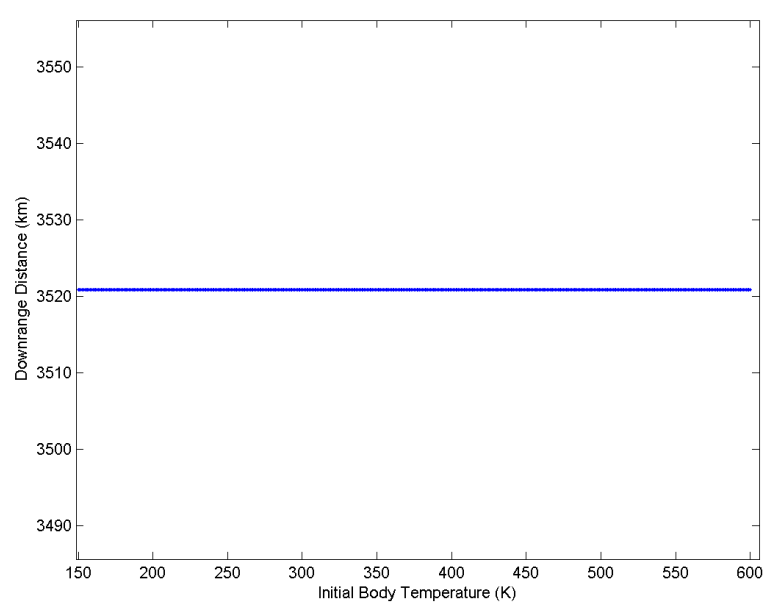

(a) Molybdenum

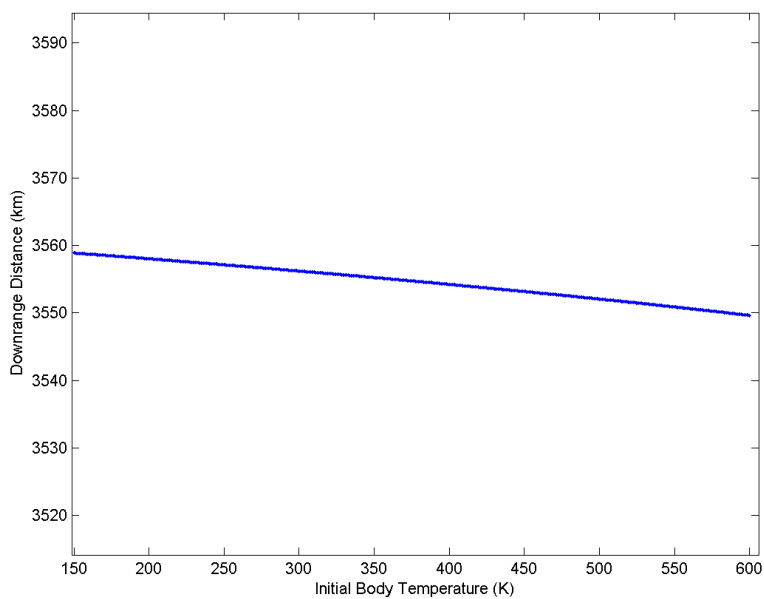

(b) Silver

Figure 5.51: Downrange Distance vs. Initial Temperature (Cylinder) 


\subsubsection{Final Mass}

The final mass is, as expected, the mass at the end of each simulated trajectory: if the object demises, the mass is zero (and the following plot appears as a horizontal line at zero); if the object survives with no ablation, the mass is the same as its initial mass on-orbit (and the following plot would appear as a horizontal line at that value). Since no molybdenum sample lost mass during the simulated trajectories and every silver sample demised, it appears that (for these two classes of materials) the final mass is insensitive to changes in initial temperature.

\subsubsection{Sphere}

Figures 5.52a and 5.52b appear similar (as horizontal lines), all the masses are identical for a material: the molybdenum samples did not lose any mass during the simulation, while all the silver samples demised before impact.

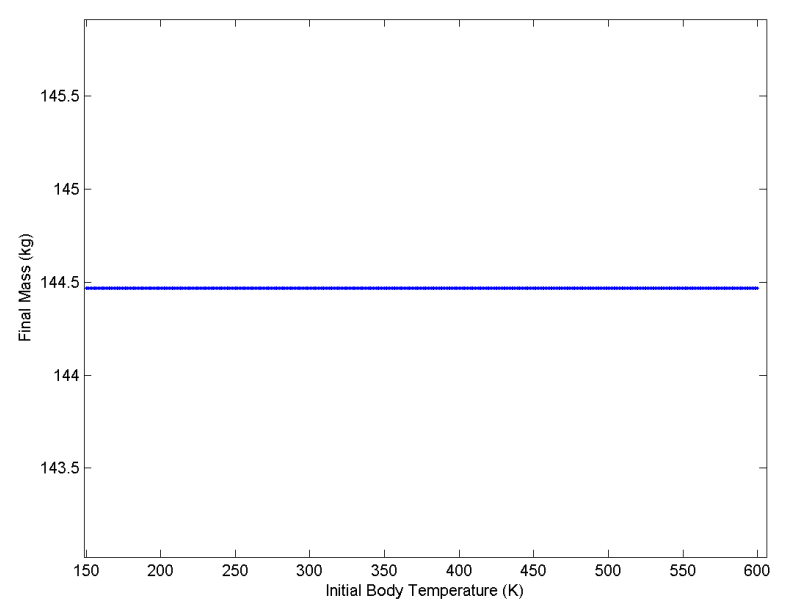

(a) Molybdenum

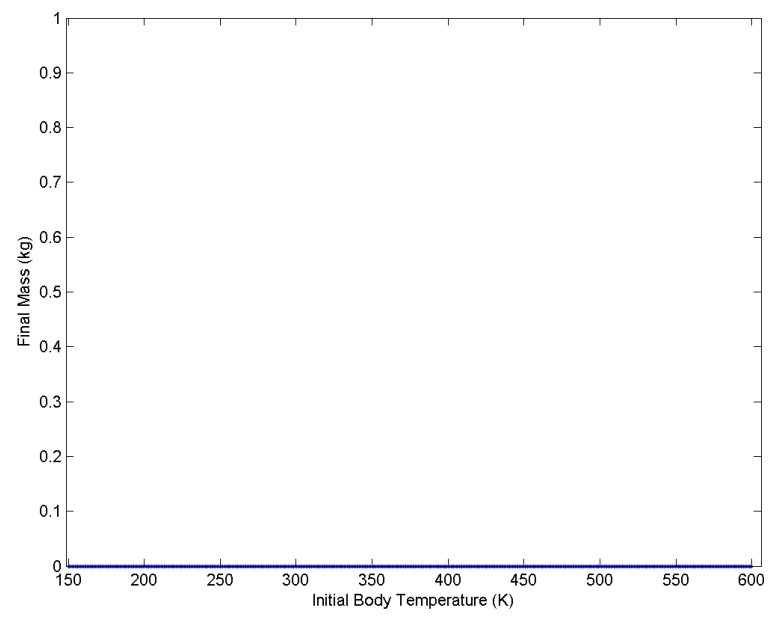

(b) Silver

Figure 5.52: Final Mass vs. Initial Temperature (Sphere)

\subsubsection{Cube}

As with the spheres, the cubes' masses did not vary with initial temperature for these materials: the molybdenum cubes lost no mass, and every silver cube demised before 
impact (seen as horizontal lines in Figures 5.53a and 5.53b, respectively).

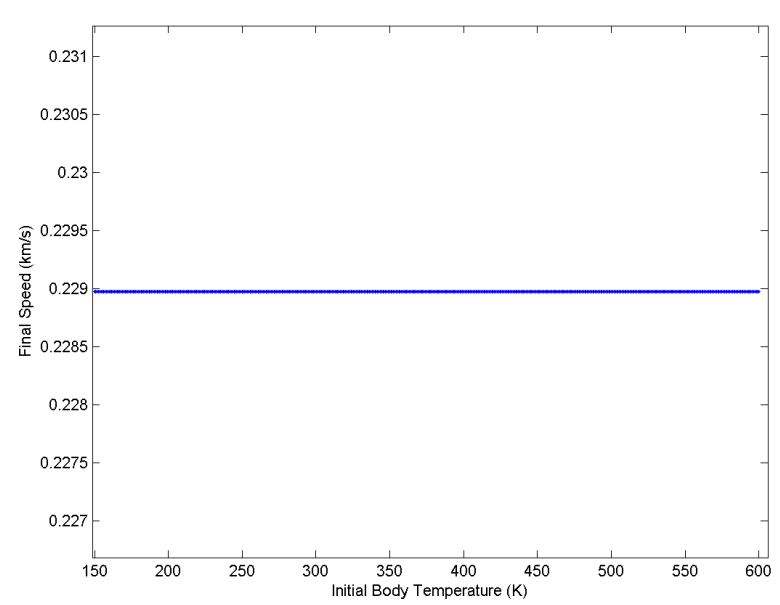

(a) Molybdenum

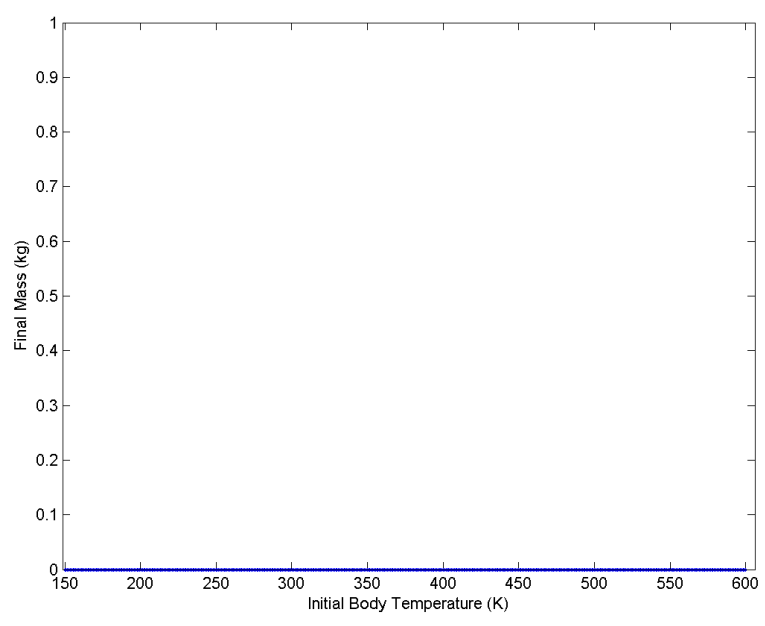

(b) Silver

Figure 5.53: Final Mass vs. Initial Temperature (Cube)

\subsubsection{Cylinder}

Cylinder final masses also appear to be insensitive to the changes in initial temperature simulated here. The horizontal lines in Figures 5.54a and 5.54b show that the molybdenum samples remain at the initial mass and the silver samples all demise, irrespective of initial body temperature.

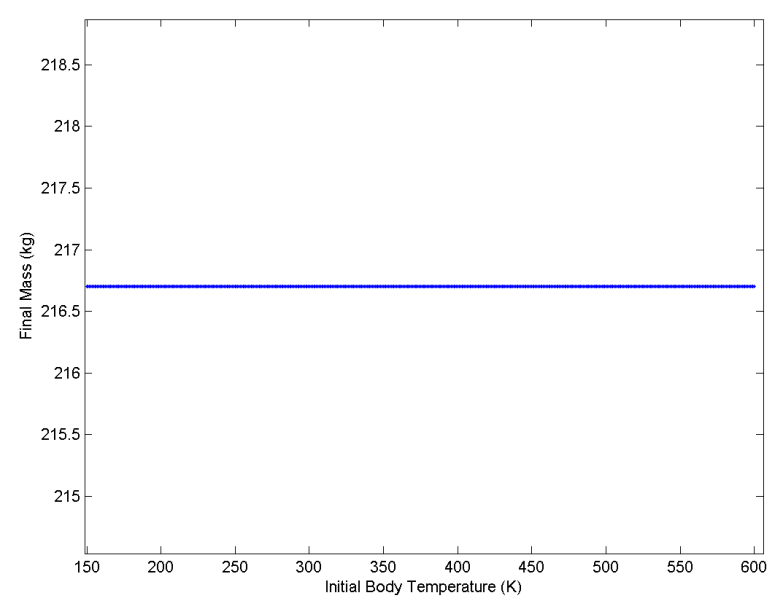

(a) Molybdenum



(b) Silver

Figure 5.54: Final Mass vs. Initial Temperature (Cylinder) 


\subsubsection{Final Speed}

The final speed is the speed at either the demise of the reentry body, or at the body's impact with the Earth's surface. The impact speed of the molybdenum samples was nearly insensitive to the initial speed, changing by less than $0.1 \%$. The silver samples also only varied by as much as $2 \%$ from the nominal speed.

\subsubsection{Sphere}

The trend in Figure 5.55a indicates that the initial temperature has no effect on final speed, and since the objects all survive to impact the surface, they are all at their terminal velocity. In contrast, Figure $5.55 \mathrm{~b}$ shows that samples that demise can have varying final speeds.

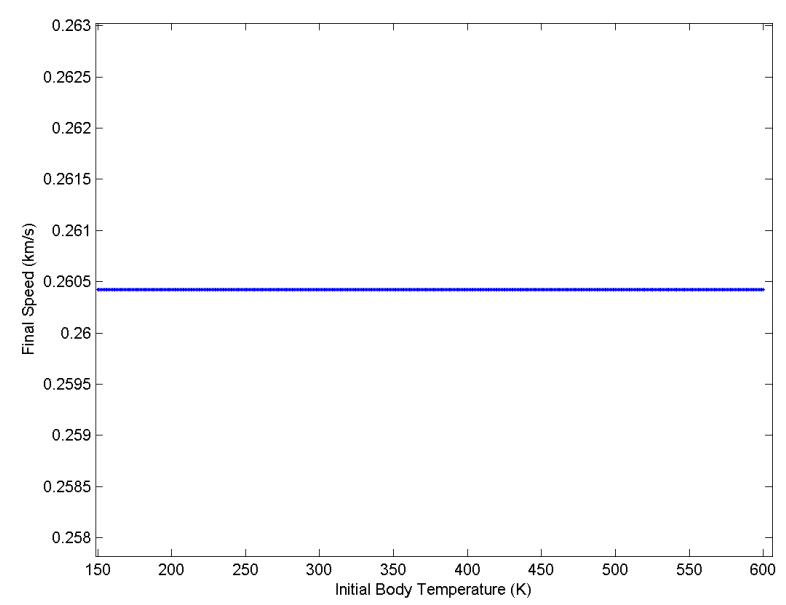

(a) Molybdenum

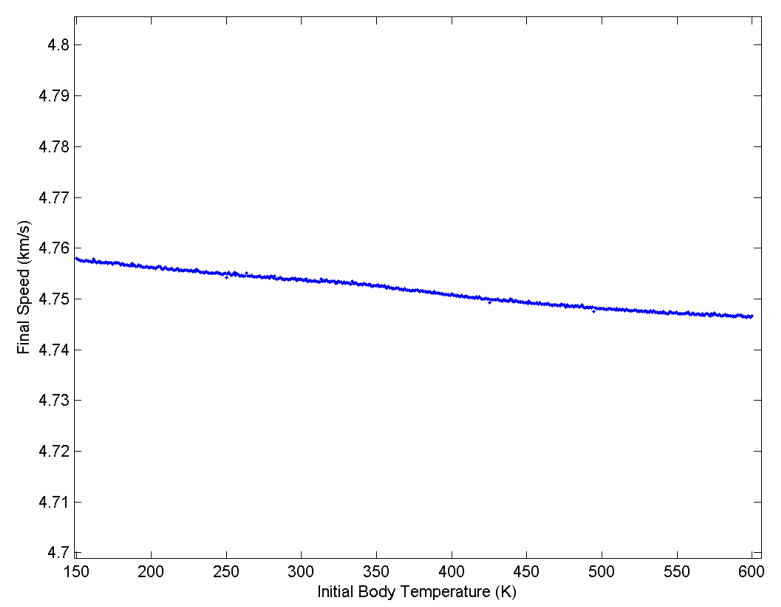

(b) Silver

Figure 5.55: Final Speed vs. Initial Temperature (Sphere)

\subsubsection{Cube}

As discussed above, the final speed of the molybdenum samples is invariant of initial temperature (see horizontal line in Figure 5.56a). The silver cubes, as with the spheres, demonstrated a weak relationship between initial temperature and final speed (see Figure $5.56 \mathrm{~b})$. 


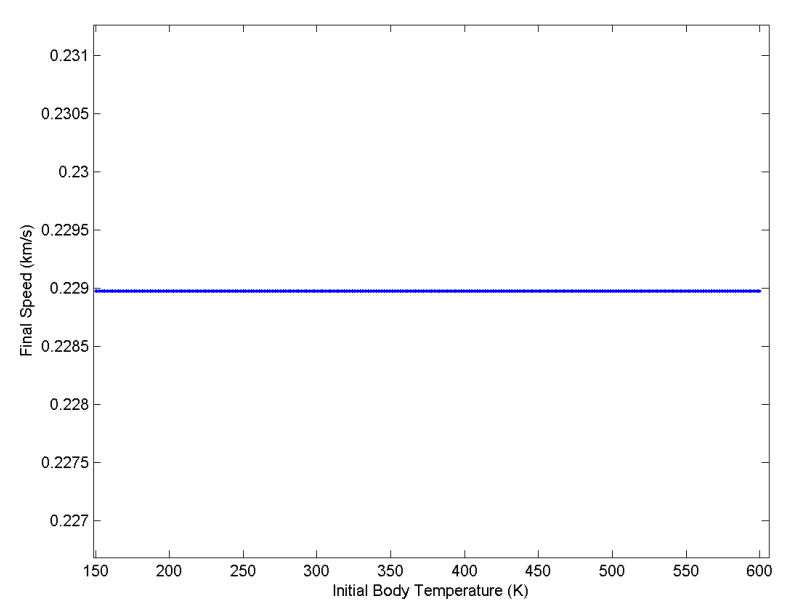

(a) Molybdenum

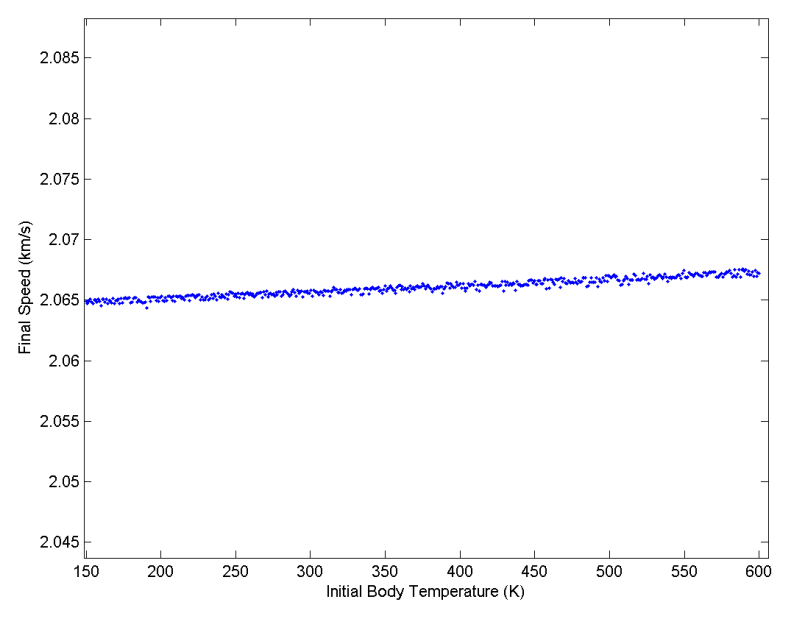

(b) Silver

Figure 5.56: Final Speed vs. Initial Temperature (Cube)

\subsubsection{Cylinder}

Again, the final speed of the molybdenum samples varies minimally with initial temperature (seen in Figure 5.33a). The final speed of the silver samples varies by up to $2 \%$ compared to the nominal temperature, as in Figure 5.33b.
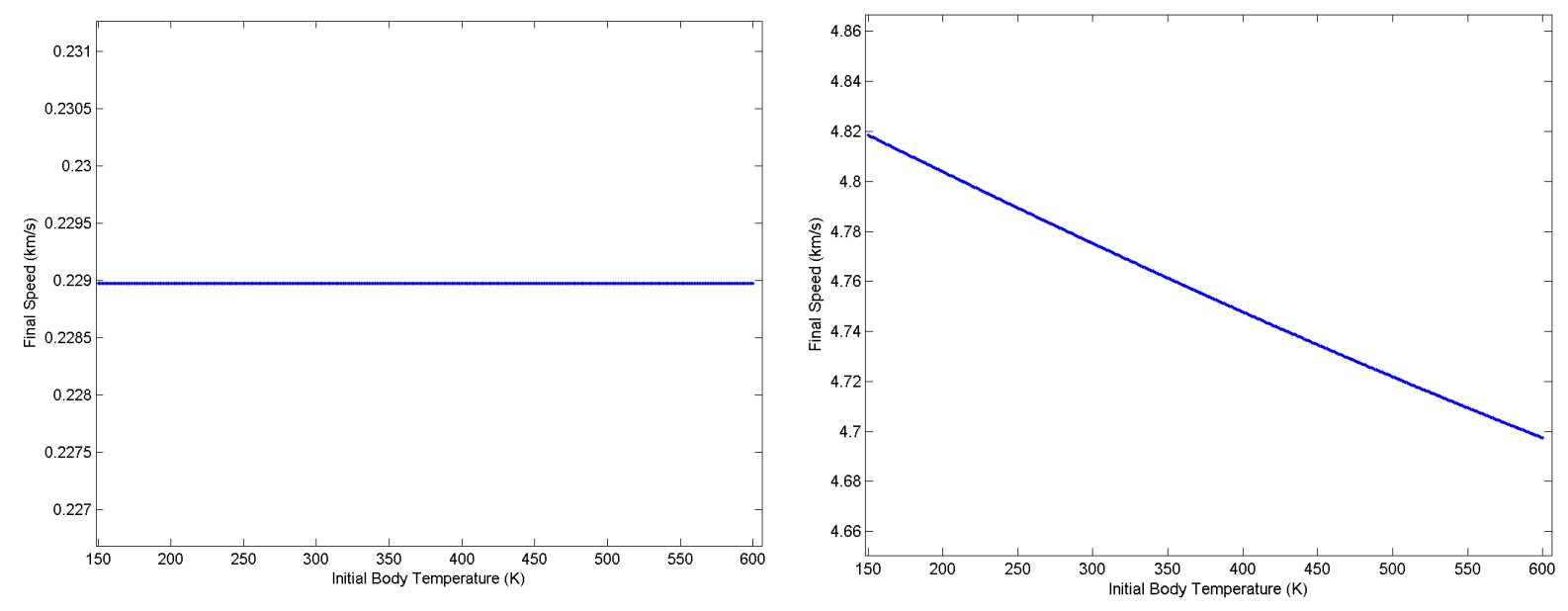

(a) Molybdenum

(b) Silver

Figure 5.57: Final Speed vs. Initial Temperature (Cylinder) 


\subsubsection{Demise Altitude}

Demise altitude is the altitude at which the mass of the reentry object reaches zero. If the object does not reach zero mass, the impact altitude (identically zero) is instead plotted here. Molybdenum samples all survived to impact regardless of initial speed in this simulation; all silver samples demised, with demise altitude depending very weakly on initial temperature - a $100 \%$ increase in temperature corresponded to a $0.1 \%$ change in demise altitude.

\subsubsection{Sphere}

Molybdenum was chosen as one of the sample materials since its physical properties caused all its mass to survive until impact with the surface (seen as a horizontal line at zero in Figure 5.58a). The demise altitude for silver varied as much as $0.1 \%$ from the nominal demise altitude, and decreased very slowly with increasing initial temperature (as seen in Figure 5.58b).

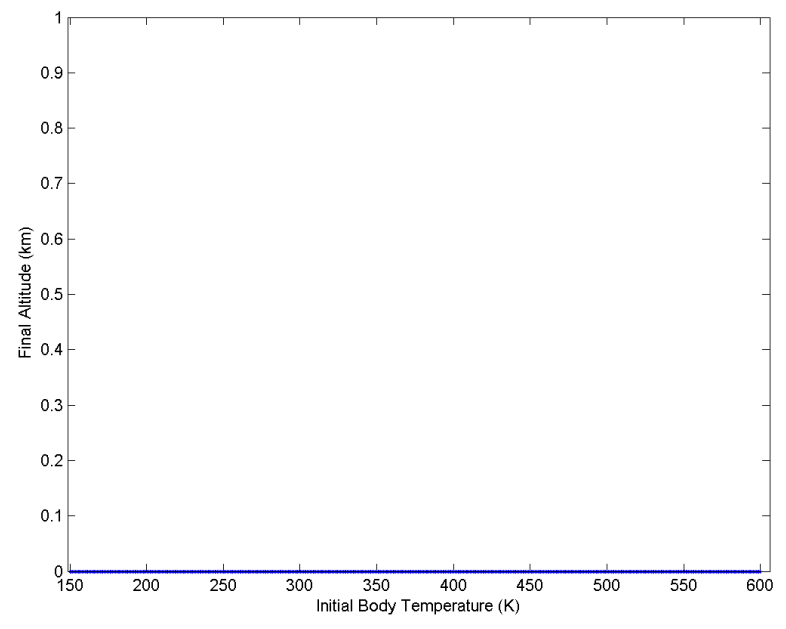

(a) Molybdenum

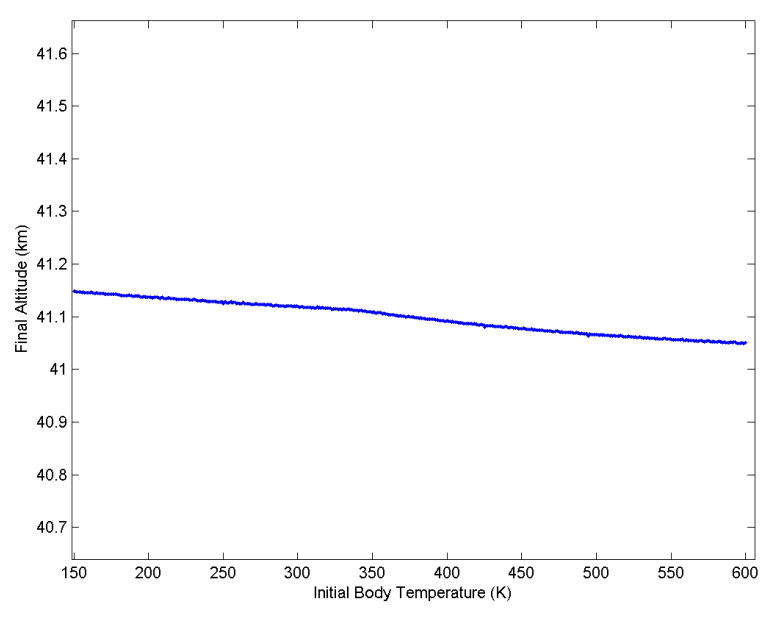

(b) Silver

Figure 5.58: Demise Altitude vs. Initial Temperature (Sphere) 


\subsubsection{Cube}

Every molybdenum cube survives reentry to impact the surface, as seen in Figure 5.59a as a horizontal line at zero. Silver cubes have demise altitudes that vary by up to $0.1 \%$ of the nominal altitude, with the demise altitude decreasing with initial temperature (see Figure 5.59b).

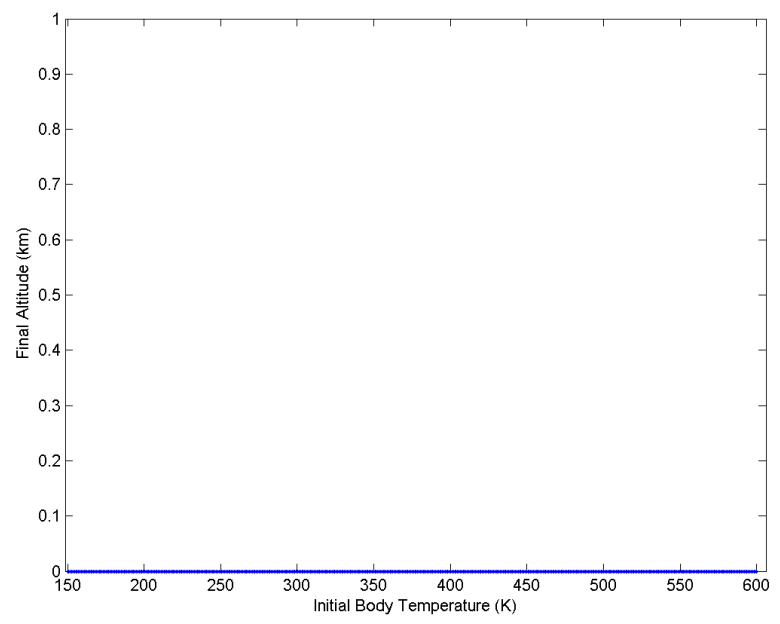

(a) Molybdenum

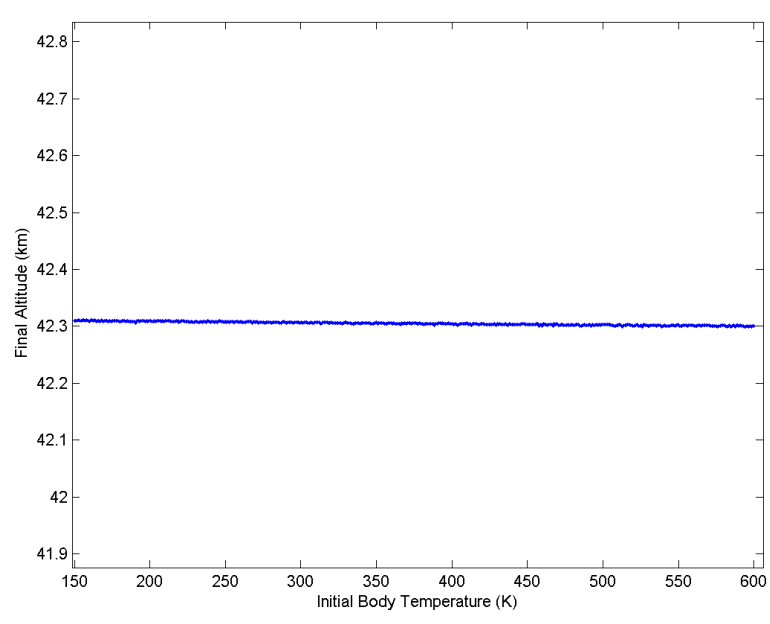

(b) Silver

Figure 5.59: Demise Altitude vs. Initial Temperature (Cube)

\subsubsection{Cylinder}

Cylinders had the same behavior as the cubes and spheres: molybdenum samples all survived reentry, and all silver samples demised, with the altitude decreasing with initial temperature (see Figures 5.60a and 5.60b, respectively). 


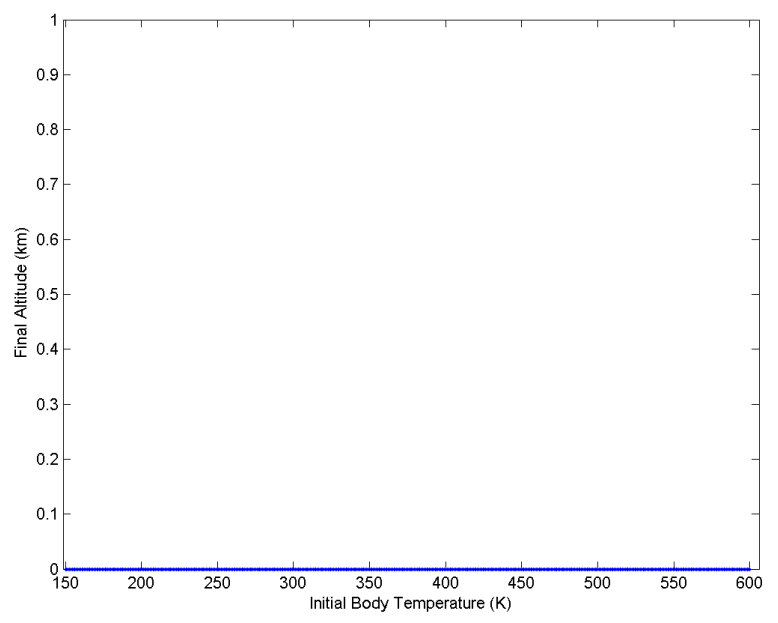

(a) Molybdenum

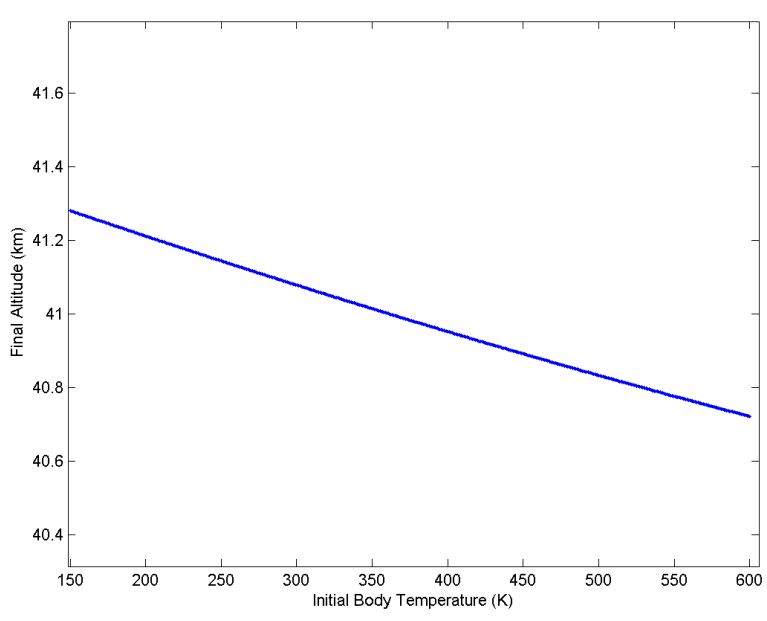

(b) Silver

Figure 5.60: Demise Altitude vs. Initial Temperature (Cylinder) 


\section{Chapter 6}

\section{Conclusion and Future Work}

\subsection{Conclusion}

A method for predicting the reentry trajectory and demise of a general object in lower Earth orbit is investigated. In the simulation, a user-defined set of initial velocity and radius vectors are used as the inputs to a simulation of its trajectory. A set of 231 different objects (77 materials in the database in three different shapes) are each run through a simulated trajectory to examine how different material properties affect demise altitude, final speed, and final mass. A sensitivity analysis in five variables was performed by simulating 500 trajectories in each of two materials and three shapes. It was noticed that downrange distance was the result with the most variation among the end results examined. Initial speed and altitude were determined as the most sensitive initial conditions, with drastic effects on demise altitude and downrange distance.

The software package offered in this thesis, OSTROM, can be used to accurately predict the demise and survival of certain solid convex objects. The discrepancy in impact kinetic energy between this thesis' code and DAS indicate further work is needed to improve the drag model at the lower altitudes and lower Mach numbers. Though the test cases presented all started at an altitude of $78 \mathrm{~km}$, the initial conditions are completely user-modifiable, and the software is capable of reasonably accurately modeling a trajectory starting as high as $140 \mathrm{~km}$.

\subsection{Future Work}

To improve the accuracy of the simulation while maintaining a lower computational cost, a table of pre-computed drag and heat flux values may be created prior to simulating any reentry trajectory. In addition, adding a 3-D conductive heat transfer model will allow

for more realistic melting and ablation (ablation more concentrated near the stagnation 
point, less so in the areas farther downstream). A materials database with temperaturevarying properties will also improve the accuracy of the simulation. A further area of interest for exploration is modeling more accurately how materials that partially ablate, like ceramics, act during reentry conditions. To improve accuracy for the materials with mid-range heats of fusion, melting points, and specific heat capacities, it will be necessary to find more data, and potentially request test cases to be run against ORSAT and SCARAB.

There is a massive potential for improved performance (or estimating error via Monte Carlo simulation of massively many trajectories simultaneously) if this software can be ported to the GPU (either via CUDA or OpenCL), which could make this suite friendlier for users who may not have access to more traditional high performance computing resources, but have a workstation with one or more GPUs. Finally, the ability to parse 3-D models with multiple parts and materials will be a crucial step in furthering the usefulness of this suite. 


\section{BIBLIOGRAPHY}

[1] Peter M. B. Waswa. Spacecraft Design-for-Demise Strategy, Analysis and Impact on Low Earth Orbit Space Missions. M.S. Thesis, Massachusetts Institute of Technology, February 2009.

[2] NASA-STD-8719.14. Process for Limiting Orbital Debris, May 2012. Revision A Change 1.

[3] IADC-2004-03. Protection Manual, January 2002.

[4] IADC-2002-01. IADC Space Debris Mitigation Guidelines, March 2004.

[5] Requirements on Space Debris Mitigation for ESA Projects. European Space Agency, April 2008.

[6] Donald L. Baradell. A Look at the Reentry Problem. AIAA Guidance and Control Conference, August 1963. AIAA Paper No. 63-318.

[7] Carmen Pardini and Luciano Anselmo. On the accuracy of satellite reentry predictions. Advances in Space Research, Vol. 34(Issue 5):1038-1043, 2004.

[8] L. N. Rowell, C. Gazley, and G. Schilling. On the Prediction of Satellite Orbit Decay and Impact. AMS/AIAA Conference on Aerospace Meteorology, (No. 66-357), March 1966.

[9] William Ailor, Lyndon Bonaparte, and Alice F. Shelton. A Strategy for Reducing Hazards from Reentry Debris. AIAA Atmospheric Flight Mechanics Conference and Exhibit, August 2006.

[10] Russell P. Patera. Hazard Analysis for Uncontrolled Space Vehicle Reentry. Journal of Spacecraft and Rockets, Vol. 45(No. 5):1031-1041, September-October 2008. 
[11] Wu Ziniu, Hu Ruifeng, Qu Xi, Wang Xiang, and Wu Zhe. Space Debris Reentry Analysis Methods and Tools. Chinese Journal of Aeronautics, Vol. 24(No. 4):387395, August 2011.

[12] Steven Millard and Felix Acon-Chen. Risk Considerations for Random Reentry of Space Debris. 48th AIAA Aerospace Sciences Meeting Including the New Horizons Forum and Aerospace Exposition, January 2010.

[13] Charles M. Reynerson. Reentry Debris Envelope Model For A Disintegrating, Deorbiting SpacecraftWithout Heat Shields. AIAA Atmospheric Flight Mechanics Conference and Exhibit, August 2005.

[14] Charles M. Reynerson. Reentry Envelope Determination Part II: Structural Failure Due To Atmospheric Heating. AIAA Atmospheric Flight Mechanics Conference and Exhibit, August 2006.

[15] Stanley A. Bouslog, Brian P. Ross, and Christopher B. Madden. Space Debris Reentry Risk Analysis. 32nd Aerospace Sciences Meeting \& Exhibit, January 1994. AIAA 94-0591.

[16] Ashish Tewari. Entry Trajectory Model with Thermomechanical Breakup. Journal of Spacecraft and Rockets, Vol. 46(No. 2):299-306, March-April 2009.

[17] A.J. Eggers Jr. NACA Report 959: One-Dimensional Flows of an Imperfect Diatomic Gas, December 1948.

[18] Tobias Lips and Bent Fritsche. A comparison of commonly used re-entry analysis tools. Acta Astronautica, Vol. 57(Nos. 2-8):312 - 323, 2005. Infinite Possibilities Global Realities, Selected Proceedings of the 55th International Astronautical Federation Congress, Vancouver, Canada, 4-8 October 2004.

[19] James E. A. John and Theo G. Keith. Gas Dynamics. Pearson Prentice Hall, 2006. 
[20] Cindi A. Karpiuk. NASA-DoD Orbital Debris Working Group Is Formed. The Orbital Debris Quarterly News, Vol. 3(No. 2):3, April 1998.

[21] R. O'Hara and M. Jansen. Overview of the NASA/JSC Debris Assessment Software (DAS) - Version 1.5. The Orbital Debris Quarterly News, Vol. 6(No. 2):5-6, April 2001.

[22] Raymond Mitchell. Orbital Debris. NASA JSC, January 2014. http://www.nasa.gov/centers/johnson/partnerships/orbital-debris/.

[23] John N. Opiela, Eric Hillary, David O. Whitlock, Marsha Hennigan, and ESCG. Debris Assessment Software User's Guide Version 2.0. NASA, January 2012.

[24] John N. Opiela and Nicholas L. Johnson. Debris Assessment Software 2.0 Now Available. The Orbital Debris Quarterly News, Vol. 12(No. 1):4, January 2008.

[25] W. Rochelle, J. Dobarco-Otero, R. Smith, R. Delaune, and K. Bledsoe. Object Reentry Survival Analysis Tool (ORSAT) - Version 6.0. The Orbital Debris Quarterly News, Vol. 10(No. 1):4, January 2006.

[26] Robert L. Kelley, Nicole M. Hill, W. C. Rochelle, Nicholas L. Johnson, and Tobias Lips. Comparison of ORSAT and SCARAB Reentry Analysis Tools for a Generic Satellite Test Case. 38th COSPAR Scientific Assembly, July 2010.

[27] Georg Koppenwallner, Bent Fritsche, Tobias Lips, and Heiner Klinkrad. SCARAB - A Multi-Disciplinary Code for Destruction Analysis of Space-Craft During ReEntry. Proceedings of the Fifth European Symposium on Aerothermodynamics for Space Vehicles, November 2005.

[28] T. Lips, V. Wartemann, G. Koppenwallner, H. Klinkrad, D. Alwes, J. DobarcoOtero, R.N. Smith, R.M. DeLaune, W.C. Rochelle, and N.L. Johnson. Comparison of 
ORSAT and SCARAB Reentry Survival Results. Proceedings of the Fourth European Conference on Space Debris, April 2005.

[29] Georg Koppenwallner, Bent Fritsche, and Tobias Lips. Multidisciplinary Analysis Tools for Orbit and Re-entry. 3rd International Workshop on Astrodynamics Tools and Techniques, October 2006.

[30] Maurice Dubin, Arnold R. Hull, and K.S.W. Champion. U.S. Standard Atmosphere, 1976. Technical Report NASA-TM-X-74335, NOAA, NASA, US Air Force, October 1976.

[31] C. G. Justus, A. W. Woodrum, R. G. Roper, and O. E. Smith. Four-D Global Reference Atmosphere Technical Description. NASA, September 1974. NASA TM X-64871.

[32] C. G. Justus, F. N. Alyea, D. M. Cunnold, W. R. Jeffries III, and D. L. Johnson. The NASA MSFC Earth Global Reference Atmospheric Model - 1990 Version. NASA, April 1991. NASA TM-4268.

[33] C. G. Justus and F. W. Leslie. The NASA MSFC Earth Global Reference Atmospheric Model - 2007 Version. NASA, November 2008. NASA/TM-2008-215581.

[34] C. G. Justus, G. R. Fletcher, F. E. Gramling, and W. B. Pace. The NASA MSFC Earth Global Reference Atmospheric Model - Mod 3 (With Spherical Harmonic Wind Model). NASA, March 1980. NASA CR-3256.

[35] C. G. Justus and D. L. Johnson. The NASA/MSFC Global Reference Atmospheric Model - 1999 Version (GRAM-99). NASA, May 1999. NASA TM-1999-209630.

[36] C. G. Justus, W. R. Jeffries III, S. P. Yung, and D. L. Johnson. The NASA MSFC Earth Global Reference Atmospheric Model - 1995 Version (GRAM-95). NASA, August 1995. NASA-TM-4715. 
[37] F. W. Leslie and C. G. Justus. The NASA Marshall Space Flight Center Earth Global Reference Atmospheric Model - 2010 Version. NASA, June 2011.

[38] L. G. Jacchia. Revised Static Models of the Thermosphere and Exosphere with Empirical Temperature Profiles. SAO Special Report 332, May 1971.

[39] A. E. Hedin, J. E. Salah, J. V. Evans, C. A. Reber, G. P. Newton, N. W. Spencer, D. C. Kayser, D. Alcayd, P. Bauer, L. Cogger, and J. P. McClure. A Global Thermospheric Model Based on Mass Spectrometer and Incoherent Scatter Data - MSIS. Journal of Geophysical Research, Vol. 82(No. 16):2139-2147, June 1977.

[40] A. E. Hedin. A Revised Thermospheric Model Based on Mass Spectrometer and Incoherent Scatter Data: MSIS-83. Journal of Geophysical Research, Vol. 88(No. A12):10170-10188, December 1983.

[41] Alan E. Hedin. MSIS-86 Thermospheric Model. Journal of Geophysical Research, Vol. 92(No. A5):4649-4662, May 1987.

[42] A. E. Hedin. Extension of the MSIS Thermosphere Model into the Middle and Lower Atmosphere. Journal of Geophysical Research, Vol. 96(No. A2):1159-1172, February 1991.

[43] J. M. Picone, A. E. Hedin, D. P. Drob, and A. C. Aikin. NRLMSISE-00 Empirical Model of the Atmosphere: Statistical Comparisons and Scientific Issues. Journal of Geophysical Research, Vol. 107(Issue A12):SIA 15-1 - SIA 15-16, December 2002.

[44] Erwin Fehlberg. Low-Order Classical Runge-Kutta Formulas with Step Size Control and their Application to some Heat Transfer Problems. Technical Report NASATR-R-315, NASA, July 1969.

[45] Erwin Fehlberg. Classical Fifth-, Sixth-, Seventh-, and Eighth-Order Runge-Kutta 
Formulas with Stepsize Control. Technical Report NASA-TR-R-287, NASA, October 1968.

[46] J. C. Butcher. Coefficients for the Study of Runge-Kutta Integration Processes. Journal of the Australian Mathematical Society, Vol. 3(No. 2):185-201, May 1963.

[47] Ernst Hairer, Syvert P. Nørsett, and Gerhard Wanner. Solving Ordinary Differential Equations I. Springer, 26th edition, 1993.

[48] Germund Dahlquist and Åke Björck. Numerical Methods. Dover Publications, Inc., Mineola, New York, 2003.

[49] F. Ceschino. Modification de la longueur du pas dans l'intégration numérique par les méthodes à pas liés. Chiffres, Vol. 2:101-106, 1961.

[50] T. E. Hull, W. H. Enright, B. M. Fellen, and A. E. Sedgwick. Comparing Numerical Methods for Ordinary Differential Equations. SIAM Journal on Numerical Analysis, Vol. 9(No. 4):603-637, December 1972.

[51] Howard D. Curtis. Orbital Mechanics for Engineering Students. Elsevier, 2nd edition, 2009.

[52] N. S. Khalifa and T. E. Sharaf-Eldin. Earth Albedo perturbations on Low Earth Orbit Cubesats. International Journal of Aeronautical and Space Sciences, Vol. 14(No. 2):193-199, June 2013.

[53] Piyush M. Mehta, Andrew Walker, Craig A. McLaughlin, and Josef Keller. Comparing physical drag coefficients computed using different gas-surface interaction models. Journal of Spacecraft and Rockets, Vol. 51(No. 3):873 - 883, April 2014.

[54] Richard G. Wilmoth, Robert A. Mitcheltree, and James N. Moss. Low-Density Aerodynamics of the Stardust Sample Return Capsule. Journal of Spacecraft and Rockets, Vol. 36(No. 3):436-441, May-June 1999. 
[55] Patrick Gallais. Atmospheric Re-Entry Vehicle Mechanics. Springer, 2007.

[56] Piyush M. Mehta, Edmondo Minisci, and Massimiliano Vasile. Sensitivity Analysis towards Probabilistic Re-Entry Modeling of Spacecraft and Space Debris. AIAA Modeling and Simulation Technologies Conference, June 2015.

[57] Graeme A. Bird. Approach to Translational Equilibrium in a Rigid Sphere Gas. Physics of Fluids, Vol. 6(No. 10):1518-1519, October 1963.

[58] Graeme A. Bird. The DSMC Method. CreateSpace, 2013. Version 1.2.

[59] Wolfgang Wagner. A Convergence Proof for Bird's Direct Simulation Monte Carlo Method for the Boltzmann Equation. Journal of Statistical Physics, Vol. 66(Nos. 3/4):1011-1044, February 1992.

[60] M. Yu. Plotnikov and E. V. Shkarupa. Estimation of the Statistical Error of the Direct Simulation Monte Carlo Method. Computational Mathematics and Mathematical Physics, Vol. 50(No. 2):335-344, February 2010.

[61] Gianluca Iaccarino. Quantification of Uncertainty in Flow Simulations Using Probabilistic Methods. AVT-162 RTO AVT/VKI Lecture Series, pages 17.1-17.30, September 2009.

[62] James N. Moss, Graeme A. Bird, and Gennady N. Markelov. DSMC Simulations of Hypersonic Flows and Comparison With Experiments. 24th International Symposium on Rarefied Gas Dynamics, July 2004.

[63] James N. Moss. Direct Simulation of Rarefied Hypersonic Flows. NASA Computational Fluid Dynamics Conference, Vol. 2(Sessions 7-12):545-558, September 1989.

[64] Thomas J. Scanlon, Craig White, Matthias Schuebler, Richard E. Brown, and Jason M. Reese. Thermochemistry Modelling in an Open-Source DSMC Code. 28th International Symposium on Shock Waves, pages 145-151, July 2011. 
[65] Graeme A. Bird. Chemical Reactions in DSMC. AIP Conference Proceedings, 1333(1):1195-1202, 2011.

[66] Raymond Brun. Kinetic Theory of Reactive Molecular Gases. AVT-162 RTO AVT/VKI Lecture Series, pages 3.1-3.20, September 2009.

[67] Wen Minhua, Lin Xinhua, and Simon Chong Wee See. GPU Based Parallel Method for Dynamic Collision Grid DSMC. Journal of Frontiers of Computer Science and Technology, Vol. 7(No. 5):472-479, 2013.

[68] M. J. Goldsworthy. A GPU-CUDA based direct simulation Monte Carlo algorithm for real gas flows. Computers \&3 Fluids, Vol. 94:58-68, May 2014.

[69] Denis Gladkov, José-Juan Tapia, Samuel Alberts, and Roshan M. D’Souza. Graphics processing unit based direct simulation Monte Carlo. SIMULATION, Vol. 88(No. 6):680-693, June 2012.

[70] Bongki Moon and Joel Saltz. Adaptive Runtime Support for Direct Simulation Monte Carlo Methods on Distributed Memory Architectures. Proceedings of the Scalable High-Performance Computing Conference, pages 176-183, May 1994.

[71] J.-S. Wu and K.-C. Tseng. Parallel DSMC method using dynamic domain decomposition. International Journal for Numerical Methods in Engineering, Vol. 63(No. 1):37-76, May 2005.

[72] P. Cremonesi, M. Ferrari, A. Frezzotti, and R. Pavani. Parallel Algorithms Applied to Direct Simulation Methods. Euromicro Workshop on Parallel and Distributed Processing, pages 239-246, January 1993.

[73] Graeme A. Bird. Recent Advances and Current Challenges for DSMC. Computers 83 Mathematics with Applications, Vol. 35(Nos. 1/2):1-14, January 1998. 
[74] Iain D. Boyd. Direct Simulation Monte Carlo for Atmospheric Entry 1. Theoretical Basis and Physical Models. AVT-162 RTO AVT/VKI Lecture Series, pages 16(1).116(1).28, September 2009.

[75] Graham V. Candler and Ioannis Nompelis. Computational Fluid Dynamics for Atmospheric Entry. AVT-162 RTO AVT/VKI Lecture Series, pages 15.1-15.56, September 2009.

[76] James N. Moss, Roop N. Gupta, and Joseph M. Price. DSMC Simulations of OREX Entry Conditions. NASA, August 1996. NASA-TM-111621.

[77] Iain D. Boyd. Direct Simulation Monte Carlo for Atmospheric Entry 2. Code Development and Application Results. AVT-162 RTO AVT/VKI Lecture Series, pages 16(2).1-16(2).28, September 2009.

[78] Chul Park. Frontiers of Aerodynamics. AVT-162 RTO AVT/VKI Lecture Series, pages 10.1-10.30, September 2009.

[79] Masoud Darbandi and Ehsan Roohi. A hybrid DSMC/NavierStokes frame to solve mixed rarefied/nonrarefied hypersonic flows over nano-plate and micro-cylinder. International Journal for Numerical Methods in Fluids, Vol. 72(No. 9):937-966, July 2013.

[80] Alexander Patronis, Duncan A. Lockerby, Matthew K. Borg, and Jason M. Reese. Hybrid continuum-molecular modelling of multiscale internal gas flows. Journal of Computational Physics, Vol. 255(No. 15):558-571, December 2013.

[81] Yu-Yung Lian, Yen-Sen Chen, K.-C. Tseng, J.-S. Wu, Bill Wu, and Luke Yang. Improved parallelized hybrid DSMC-NS method. Computers \& Fluids, Vol. 45(No. 1):254-260, June 2011. 
[82] Zbigniew Koza, Andrzej Kosior, Maciej Matyka, Jakub Pola, and Lukasz Miroslaw. Multi-GPU simulations in OpenFOAM with SpeedIT technology. Workshop Presentation, November 2011. http://www.vratis.com/blog/.

[83] T. J. Scanlon, E. Roohi, C. White, M. Darbandi, and J. M. Reese. An open source, parallel DSMC code for rarefied gas flows in arbitrary geometries. Computers 83 Fluids, Vol. 39(No. 10):2078-2089, December 2010.

[84] Erik Arlemark, Gennady Markelov, and Silvia Nedea. Rebuilding of Rothe's nozzle measurements with OpenFOAM software. 1st European Conference on Gas Micro Flows, June 2012.

[85] Nishanth Dongari, Craig White, Thomas J. Scanlon, Yongzhao Zhang, and Jason M. Reese. Effects of curvature on rarefied gas flows between rotating concentric cylinders. Physics of Fluids, Vol. 25(No. 5):052003.1-052003.17, May 2013.

[86] Abdul O. Ahmad, Thomas J. Scanlon, and Jason M. Reese. Benchmarking a New, Open-Source Direct Simulation Monte Carlo (DSMC) Code for Hypersonic Flows. 28th International Symposium on Shock Waves, pages 1081-1087, July 2012.

[87] Klas Jaretag. Coupled calculations in OpenFOAM. 2nd Gothenburg Region OpenFOAM User Group Meeting, November 2012.

[88] Hrvoje Jasak. Coupled Multiphysics with OpenFOAM. 7th OpenFOAM Workshop, June 2012.

[89] S. Pantazis and H. Rusche. A hybrid continuum-particle solver for unsteady rarefied gas flows. Vacuum, Vol. 109:275-283, November 2014.

[90] T. F. Zien. Recent Developments in Modeling Hypersonic Melting Ablation. 6th AIAA Theoretical Fluid Mechanics Conference, June 2011. AIAA 2011-3304. 
[91] Wilbur L. Hankey. Re-Entry Aerodynamics. American Institute of Aeronautics and Astronautics, 1988. 
APPENDICES 


\section{Appendix A}

\section{Nomenclature}

\begin{tabular}{|c|c|c|}
\hline Symbol & Units & Description \\
\hline$\beta$ & $\frac{\mathrm{kg}}{\mathrm{m}^{2}}$ & Ballistic coefficient \\
\hline$\gamma$ & - & Specific heat ratio \\
\hline$\epsilon$ & $\frac{\mathrm{km}^{2}}{\mathrm{~s}}$ & Specific orbital energy \\
\hline$\lambda$ & $\mathrm{m}$ & Mean free path \\
\hline$\rho$ & $\frac{\mathrm{kg}}{\mathrm{m}^{3}}$ & Mass density \\
\hline$\mu$ & $\mathrm{Pa} \cdot \mathrm{s}$ & Dynamic viscosity \\
\hline$\mu$ & $\frac{\mathrm{km}^{3}}{\mathrm{~s}^{2}}$ & Standard gravitational parameter \\
\hline$C_{D}$ & - & Drag coefficient \\
\hline$H$ & $\mathrm{~km}$ & Scale height \\
\hline$L$ & $\mathrm{~m}$ & Length \\
\hline$L$ & $\frac{\mathrm{K}}{\mathrm{m}}$ & Temperature lapse rate \\
\hline$K n$ & - & Knudsen number \\
\hline$M$ & - & Mach number \\
\hline$M$ & $\frac{\mathrm{kg}}{\mathrm{mol}}$ & Molar mass \\
\hline$N_{A}$ & $\mathrm{~mol}^{-1}$ & Avogadro's number \\
\hline$\dot{Q}$ & $\frac{\mathrm{J}}{\mathrm{s}}$ & Heating rate \\
\hline$R$ & $\frac{\mathrm{J}}{\mathrm{kg} \cdot \mathrm{K}}$ & Universal Gas Constant \\
\hline$S_{r e f}$ & $\mathrm{~m}^{2}$ & Reference area \\
\hline$S t$ & - & Stanton number \\
\hline$T$ & K & Temperature \\
\hline$T_{\text {melt }}$ & $\mathrm{K}$ & Melting point temperature \\
\hline$a$ & $\frac{\mathrm{m}}{\mathrm{s}}$ & Acoustic velocity \\
\hline
\end{tabular}




\begin{tabular}{|c|c|c|}
\hline$c_{p}$ & $\frac{\mathrm{J}}{\mathrm{kg} \cdot \mathrm{K}}$ & Specific heat capacity \\
\hline$c_{p}$ & - & Pressure coefficient \\
\hline$d$ & $\mathrm{~m}$ & Particle hard shell diameter \\
\hline$e$ & $\mathrm{~J}$ & Internal energy \\
\hline$g$ & $\frac{\mathrm{m}}{\mathrm{s}^{2}}$ & Acceleration due to gravity \\
\hline$h$ & $\mathrm{~m}$ & Altitude \\
\hline$h$ & s & Integration step size \\
\hline$h_{f}$ & $\frac{\mathrm{J}}{\mathrm{kg}}$ & Specific heat of fusion \\
\hline$k_{B}$ & $\frac{\mathrm{J}}{\mathrm{K}}$ & Boltzmann constant \\
\hline$m$ & $\mathrm{~kg}$ & Mass \\
\hline$\dot{m}$ & $\frac{\mathrm{kg}}{\mathrm{s}}$ & Mass flow rate \\
\hline$p$ & $\mathrm{~Pa}$ & Static pressure \\
\hline$\vec{p}$ & $\frac{\mathrm{m}}{\mathrm{s}^{2}}$ & Perturbation accleration vector \\
\hline$q$ & $\mathrm{~Pa}$ & Dynamic pressure \\
\hline$r$ & $\mathrm{~km}$ & Magnitude of $\vec{r}$ \\
\hline$\vec{r}$ & $\mathrm{~km}$ & Vector from center of Earth to object \\
\hline$u$ & $\frac{\mathrm{J}}{\mathrm{kg}}$ & Specific internal energy \\
\hline$v$ & $\frac{\mathrm{m}}{\mathrm{s}}$ & Magnitude of $\vec{v}$ \\
\hline$\vec{v}$ & $\frac{\mathrm{m}}{\mathrm{s}}$ & Vector velocity of object \\
\hline$y$ & - & Gas component fraction \\
\hline$y$ & - & State vector from predictor \\
\hline$\hat{y}$ & - & State vector from corrector \\
\hline
\end{tabular}

\section{Subscripts}

$\infty$

0

0

C
Freestream condition

Stagnation point condition

Sea-Level Standard condition

Continuum 
$F M$

$s$

$x$

$y$

$z$

Free Molecular

Surface condition

X-component

Y-component

Z-component 


\section{Appendix B}

case.txt

// case file for running one instance of OSTROM

$/ / \operatorname{rvec}(\mathrm{km})$

$/ / \operatorname{vvec}(\mathrm{km} / \mathrm{s})$

// \% uncertainty to add in rx, ry, rz (1-sigma values)

// \% uncertainty to add in vx, vy, vz (1-sigma values)

$/ / \operatorname{mass}(\mathrm{kg})$

// Material

// Shape

$3228.00 .0-5591.0$

0.07 .8530 .0

0.0010 .0010 .001

0.50 .50 .5

16.54

Acrylic

Sphere 


\section{Appendix C}

\section{Simulation Geometries}

\begin{tabular}{|c|c|c|c|c|}
\hline Variable & Sphere & Cube & Cylinder & Description (Units) \\
\hline $\mathrm{L}$ & & 0.3 & 0.6 & Length $(m)$ \\
$\mathrm{r}$ & 0.15 & & 0.15 & Base radius $(m)$ \\
$\mathrm{A}$ & $4 \pi r^{2}$ & $6 L^{2}$ & $2 \pi r^{2}+2 \pi r L$ & Surface Area $\left(m^{2}\right)$ \\
$\mathrm{V}$ & $\frac{4}{3} \pi r^{3}$ & $L^{3}$ & $\pi r^{2} L$ & Volume $\left(m^{3}\right)$ \\
$S_{r e f}$ & $\pi r^{2}$ & $L^{2}$ & $\pi r^{2}$ & Reference Area $\left(m^{2}\right)$ \\
\hline
\end{tabular}




\section{Appendix D}

\section{Materials Database}

\section{Reproduced from [23]}

\begin{tabular}{|c|c|c|c|c|}
\hline Material Name & Specific Heat (J/kg-K) & Heat of Fusion $(\mathrm{J} / \mathrm{kg})$ & Melt Temperature (K) & Density $\left(\mathrm{kg} / \mathrm{m}^{3}\right)$ \\
\hline Acrylic & 1465 & 0.0 & 505.0 & 1170.00 \\
\hline Alumina & $790.6-1231.4$ & 106757.0 & 2305.4 & 3990.00 \\
\hline Aluminum (generic) & 1100 & 390000.0 & 850.0 & 2700.00 \\
\hline Aluminum 1145-H19 & 904 & 386116.0 & 919.0 & 2697.00 \\
\hline Aluminum 2024 - T3 & 972.7 & 386116.0 & 856.0 & 2803.20 \\
\hline Aluminum 2024-T8xx & $845.3-1100.0$ & 386116.0 & 856.0 & 2803.00 \\
\hline Aluminum 2219-T8xx & $866.0-1147.2$ & 386116.0 & 867.0 & 2812.80 \\
\hline Aluminum 5052 & 900.2 & 386116.0 & 880.0 & 2684.90 \\
\hline Aluminum 6061-T6 & 896 & 386116.0 & 867.0 & 2707.00 \\
\hline Aluminum 7075-T6 & $846.8-1177.9$ & 376788.0 & 830.0 & 2787.00 \\
\hline Barium element & 285 & 55824.0 & 983.0 & 3492.00 \\
\hline Beryllium element & $1675.4-3594.8$ & 1093220.0 & 1557.0 & 1842.00 \\
\hline Beta cloth & 837.5 & 232.6 & 650.0 & 1581.00 \\
\hline Brass, Red & $397.7-410.3$ & 195372.0 & 1280.0 & 8746.00 \\
\hline Brass, Cartridge & $397.7-414.5$ & 179091.0 & 1208.0 & 8521.80 \\
\hline Brass, Muntz & $397.7-427.0$ & 167461.0 & 1174.0 & 8393.67 \\
\hline Carbon-Carbon Reinforced & $785.1-1730.0$ & 37650.0 & 2144.0 & 1688.47 \\
\hline Cobalt & $412.4-904.5$ & 259600.0 & 1768.0 & 8862.00 \\
\hline Cork & 1629.2 & 2860980.0 & 922.0 & 261.29 \\
\hline $\mathrm{Cu}$ alloy & $389.4-471.8$ & 204921.0 & 1356.0 & 8938.00 \\
\hline $\mathrm{Cu} / \mathrm{Be}(0.5 \% \mathrm{Be})$ & 397 & 204921.0 & 1320.0 & 8800.00 \\
\hline $\mathrm{Cu} / \mathrm{Be}(1.9 \% \mathrm{Be})$ & $253.0-652.0$ & 204921.0 & 1199.0 & 8248.60 \\
\hline Fiberfrax & 1130.5 & 0.0 & 2089.0 & 96.10 \\
\hline Fiberglass & 1046.8 & 232.6 & 1200.0 & 1840.35 \\
\hline FRCI-12 shuttle tiles & $705.3-1273.6$ & 0.0 & 1922.0 & 192.22 \\
\hline Gallium Arsenide & 325 & 0.0 & 1510.0 & 5316.00 \\
\hline Germanium & $329.7-397.7$ & 430282.6 & 1210.7 & 5320.00 \\
\hline Gold element & $126.4-153.3$ & 64895.0 & 1336.0 & 19300.00 \\
\hline Graphite epoxy 1 & 879 & 232.6 & 700.0 & 1550.50 \\
\hline Graphite epoxy 2 & 879 & 232.6 & 700.0 & 1550.50 \\
\hline Hastelloy c & $498.4-694.6$ & 309803.0 & 1620.0 & 8920.67 \\
\hline Hastelloy 25 & $406.2-590.0$ & 309803.0 & 1643.0 & 9130.00 \\
\hline Hastelloy 188 & $406.2-590.0$ & 309803.0 & 1635.0 & 8980.00 \\
\hline Hastelloy n & $419.0-584.4$ & 309803.0 & 1623.0 & 8576.40 \\
\hline Inconel $\mathrm{x}$ & $426.3-541.8$ & 311664.0 & 1683.2 & 8297.50 \\
\hline Inconel 600 & $426.7-650.2$ & 297206.0 & 1683.9 & 8415.00 \\
\hline Inconel 601 & $449.9-815.9$ & 311664.0 & 1659.0 & 8057.29 \\
\hline Inconel 625 & 410 & 311664.0 & 1593.0 & 8440.00 \\
\hline Inconel 718 & 435 & 311664.0 & 1571.0 & 8190.00 \\
\hline Invar & $479.9-653.2$ & 2740000.0 & 1700.0 & 8050.00 \\
\hline Iron (Armco) & $433.7-711.5$ & 272125.0 & 1812.0 & 7865.00 \\
\hline Lead element & $130.5-138.8$ & 23958.0 & 600.0 & 11677.00 \\
\hline Macor ceramic & 790 & 236850.0 & 1300.0 & 2520.00 \\
\hline Magnesium AZ31 & $1027.3-1398.3$ & 339574.0 & 868.0 & 1682.00 \\
\hline Magnesium HK31A & $1028.9-1340.6$ & 325619.0 & 877.0 & 1794.00 \\
\hline Molybdenum & $231.7-412.0$ & 293057.0 & 2899.0 & 10219.00 \\
\hline MLI & 1046.6 & 232.6 & 617.0 & 772.48 \\
\hline
\end{tabular}




\begin{tabular}{|c|c|c|c|c|}
\hline MP35N & $440.0-726.0$ & 309803.0 & 1650.0 & 8430.00 \\
\hline Nickel & $440.0-726.7$ & 309803.0 & 1728.2 & 8906.26 \\
\hline Niobium & $268.8-346.5$ & 290000.0 & 2741.0 & 8570.00 \\
\hline NOMEX & 1256 & 232.6 & 572.0 & 1380.00 \\
\hline Platinum & $130.7-146.2$ & 113967.0 & 2046.4 & 21448.70 \\
\hline Polyamide & 1130 & 232.6 & 723.0 & 1420.00 \\
\hline Polycarbonate (Lexan) & 1260 & 0.0 & 573.0 & 1250.00 \\
\hline RCG Coating & $814.7-1633.7$ & 0.0 & 1922.0 & 1665.91 \\
\hline Rene41 & $346.9-914.9$ & 311664.0 & 1728.0 & 8249.00 \\
\hline Silver element & $222.6-243.7$ & 105833.0 & 1234.0 & 10492.00 \\
\hline Sodium-Iodide & 84 & 290759.6 & 924.0 & 3470.00 \\
\hline Stainless Steel (generic) & 600 & 270000.0 & 1700.0 & 7800.00 \\
\hline Steel $17-4 \mathrm{ph}$ & 666.8 & 286098.0 & 1728.0 & 7833.03 \\
\hline Steel 21-6-9 & 439 & 286098.0 & 1728.0 & 7832.80 \\
\hline Steel A-286 & 460.6 & 286098.0 & 1644.0 & 7944.90 \\
\hline Steel AISI 304 & $482.0-608.2$ & 286098.0 & 1700.0 & 7900.00 \\
\hline Steel AISI 316 & 460.6 & 286098.0 & 1644.0 & 8026.85 \\
\hline Steel AISI 321 & $565.2-651.2$ & 286098.0 & 1672.0 & 8026.60 \\
\hline Steel AISI 347 & $471.9-638.0$ & 286098.0 & 1686.0 & 7960.00 \\
\hline Steel AISI 410 & 485.7 & 286098.0 & 1756.0 & 7749.50 \\
\hline Strontium element & 737 & 95599.0 & 1043.0 & 2595.00 \\
\hline Teflon & 1674 & 0.0 & 533.0 & 2162.50 \\
\hline Titanium (generic) & 600 & 470000.0 & 1950.0 & 4400.00 \\
\hline Titanium (6 Al-4 V) & $500.4-1114.6$ & 393559.0 & 1943.0 & 4437.00 \\
\hline Tungsten & $127.1-188.0$ & 220040.0 & 3650.0 & 19300.00 \\
\hline Uranium & $116.8-201.1$ & 52523.0 & 1405.0 & 19099.00 \\
\hline Uranium Zirconium Hydride & 418.7 & 131419.0 & 2144.0 & 6086.80 \\
\hline Water & $4081.1-6900.0$ & 0.1 & 273.0 & 999.00 \\
\hline Zerodur & $842.4-1644.7$ & 250000.0 & 1424.0 & 2530.00 \\
\hline Zinc & $366.6-444.0$ & 100942.0 & 692.6 & 7144.20 \\
\hline
\end{tabular}




\section{Appendix E}

\section{DAS Test Case Results}

\begin{tabular}{|c|c|c|c|c|c|}
\hline Material & Shape & Mass $(\mathrm{kg})$ & Casualty Area $\left(\mathrm{m}^{2}\right)$ & Demise Alt. $(\mathrm{km})$ & $\mathrm{KE}(\mathrm{MJ})$ \\
\hline Acrylic & Sphere & 16.54 & 0 & 73.9 & \\
Acrylic & Cube & 31.59 & 0 & 74.4 & \\
Acrylic & Cylinder & 49.62 & 0 & 73.3 & \\
Molybdenum & Sphere & 144.468 & 0.75 & & 6.001 \\
Molybdenum & Cube & 275.913 & 0.81 & & 10.299 \\
Molybdenum & Cylinder & 433.403 & 1.05 & & \\
Silver & Sphere & 148.327 & 0 & 43.2 & \\
Silver & Cube & 283.284 & 0 & 41.2 & \\
Silver & Cylinder & 444.981 & 0 & 43.3 & \\
\hline
\end{tabular}

